\title{
A UML Profile For Developing Airworthiness-Compliant (RTCA DO-178B) Safety-Critical Software
}

By

\author{
Gregory Zoughbi
}

\author{
A thesis submitted to \\ The Faculty of Graduate Studies and Research \\ In partial fulfillment of the requirements of the degree of \\ Master's of Applied Science
}
Ottawa-Carleton Institute of Electrical and Computer Engineering
Department of Systems and Computer Engineering
Carleton University
Ottawa, Ontario, K1S 5B6

Canada

December 2006

Copyright @ 2006 by Gregory Zoughbi 


$\begin{array}{ll}\begin{array}{l}\text { Library and } \\ \text { Archives Canada }\end{array} & \begin{array}{l}\text { Bibliothèque et } \\ \text { Archives Canada }\end{array} \\ \begin{array}{l}\text { Published Heritage } \\ \text { Branch }\end{array} & \begin{array}{l}\text { Direction du } \\ \text { Patrimoine de l'édition }\end{array} \\ \begin{array}{l}\text { 395 Wellington Street } \\ \text { Ottawa ON K1A ON4 }\end{array} & \begin{array}{l}\text { 395, rue Wellington } \\ \text { Ottawa ON K1A ON4 } \\ \text { Canada }\end{array}\end{array}$

Your file Votre référence ISBN: 978-0-494-23353-5 Our file Notre référence ISBN: 978-0-494-23353-5

NOTICE:

The author has granted a nonexclusive license allowing Library and Archives Canada to reproduce, publish, archive, preserve, conserve, communicate to the public by telecommunication or on the Internet, loan, distribute and sell theses worldwide, for commercial or noncommercial purposes, in microform, paper, electronic and/or any other formats.

The author retains copyright ownership and moral rights in this thesis. Neither the thesis nor substantial extracts from it may be printed or otherwise reproduced without the author's permission.
AVIS:

L'auteur a accordé une licence non exclusive permettant à la Bibliothèque et Archives Canada de reproduire, publier, archiver, sauvegarder, conserver, transmettre au public par télécommunication ou par l'Internet, prêter, distribuer et vendre des thèses partout dans le monde, à des fins commerciales ou autres, sur support microforme, papier, électronique et/ou autres formats.

L'auteur conserve la propriété du droit d'auteur et des droits moraux qui protège cette thèse. $\mathrm{Ni}$ la thèse ni des extraits substantiels de celle-ci ne doivent être imprimés ou autrement reproduits sans son autorisation.
In compliance with the Canadian

Privacy Act some supporting forms may have been removed from this thesis.

While these forms may be included in the document page count, their removal does not represent any loss of content from the thesis.
Conformément à la loi canadienne sur la protection de la vie privée, quelques formulaires secondaires ont été enlevés de cette thèse.

Bien que ces formulaires aient inclus dans la pagination, il n'y aura aucun contenu manquant.

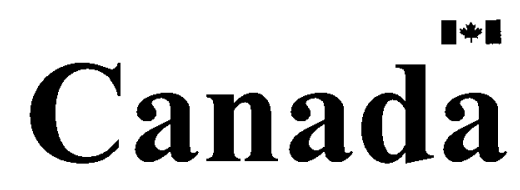




\section{ABSTRACT}

Many safety-related and certification standards exist for developing safety-critical systems. Safety assessments are performed in practice, and system certification according to a standard requires the submitting information about the software. The airworthiness standard, RTCA DO-178B, is the software de-facto standard for commercial and military aerospace programmes. The objective of this research is to propose an approach to improve the line of communication between safety engineers and software engineers by proposing a Unified Modeling Language (UML) profile that allows software engineers to model safety related concepts and properties in UML, the de-facto software modeling language. In this research, the list of safety-related concepts is extracted from RTCA DO178B, and then a UML profile is presented to enable modeling them. Then, approaches to generate certification-related information from UML models are presented. This new approach is illustrated through a case study on developing an aircraft's navigation controller subsystem.

Keywords: UML, UML Profile, Airworthiness, RTCA DO-178B, Safety, SafetyCritical, Safety Assessment, Certification, Certification Authority. 


\section{ACKNOWLEDGEMENTS}

I would like to express sincere gratitude for all those who have helped me throughout this thesis. First and foremost, I would like to thank my parents Hiyam and Saleem, and my brother Michael, for continuously and unconditionally supporting me throughout my life. They have always encouraged me to pursue career-defining opportunities, and the successful completion of this thesis is no different. I would not have been able to complete this work without them.

I would like to thank my thesis supervisors, Dr. Lionel Briand and Dr. Yvan Labiche. They have guided me throughout my master's degree by helping me select appropriate courses, developing my interest in safety-critical systems, and most importantly working closely with me to significantly increase the quality of this thesis. Their constructive feedback and input was instrumental for this research.

In addition, I would like to thank Dr. Fred Cotaras of General Dynamics (GD) Canada for helping me define the research problem and relate it to practical needs of engineering organizations. Fred helped me take the first, and most challenging, step of this journey.

Last but not least, I would like to thank Mr. Christopher Sean Elliott, my software engineering mentor at the Aurora Data Management System (DMS) project at GD Canada, for coaching me on how to design high-quality software for large and complex software systems. Chris has provided me with effective software engineering skills, which he acquired through decades of experience developing large software systems such 
as the Canadian Automated Air Traffic System (CAATS) - a software system which, according to Chris, has "one of the finest architectures ever devised". Those skills were vital in the design of the UML profile in this thesis.

Thank you all!

Gregory Zoughbi

December 2006

Ottawa, Canada 


\section{TABLE OF CONTENTS}

1 INTRODUCTION 1

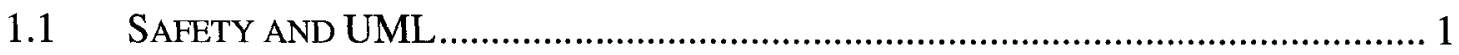

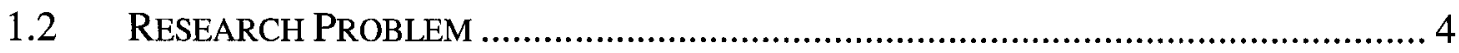

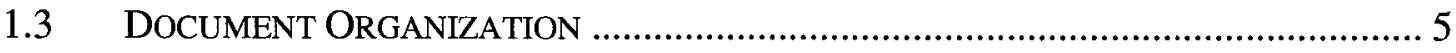

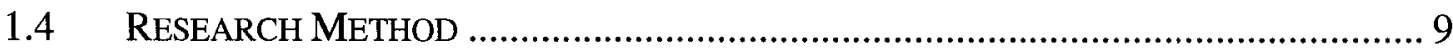

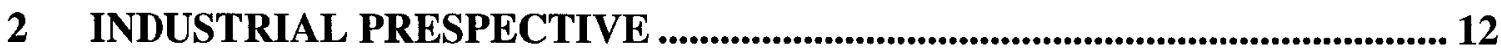

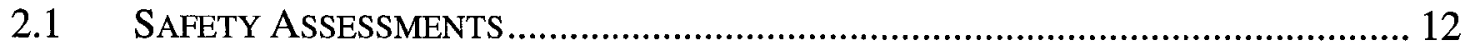

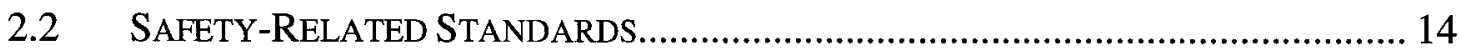

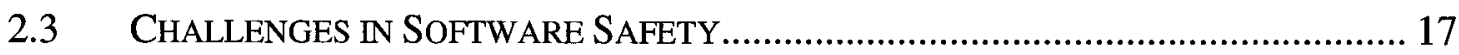

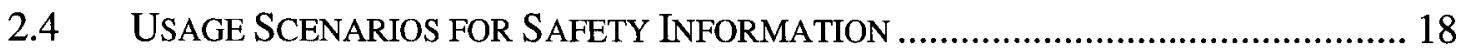

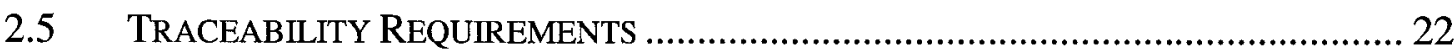

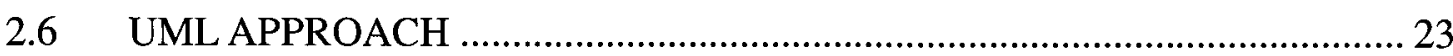

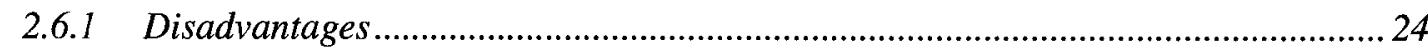

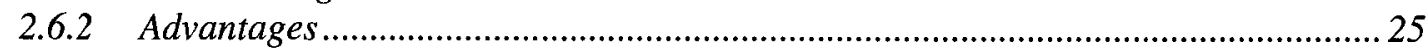

2.6.3 Requirements of an Effective UML Profile ..........................................................2 28

3 SAFETY-RELATED CONCEPTS .......................................................................... 31

3.1 CONCEPT IDENTIFICATION AND CATEGORIZATION ……...................................... 34

3.2 CONCEPT REFINEMENT ................................................................................. 41

3.2.1 Conceptual Model ................................................................................ 43

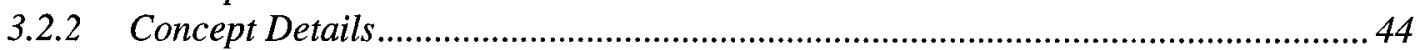

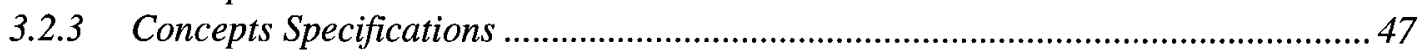

3.2.4 Providing Traceability .............................................................................. 73

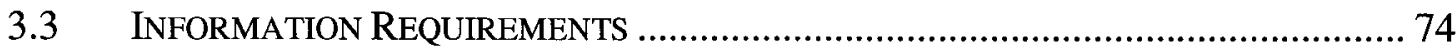

4 EXISTING UML PROFILES............................................................................ 82

4.1 QUALITY OF SERVICE AND FAULT TOLERANCE OMG PROFILE ……….............. 82

4.2 SCHEDULABILITY, PERFORMANCE, AND TIME OMG PROFILE …………………... 83

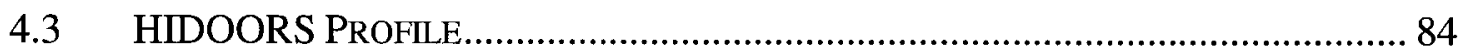

4.4 EFFECTS OF MESSAGE LOSS, DELAY, AND CORRUPTION ................................... 85

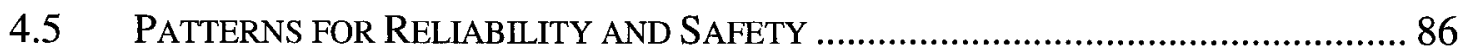

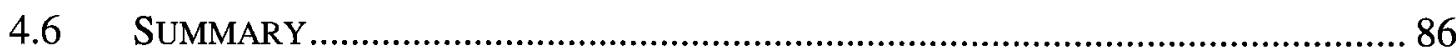

5 PROPOSED UML PROFILE............................................................................. 89

5.1 UML PROFILE-TEMPLATE DESCRIPTION............................................................. 89

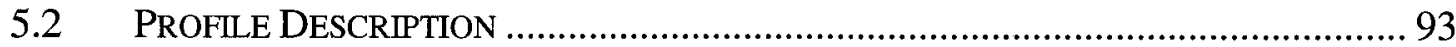

$5.2 .1<<$ SafetyContext>>

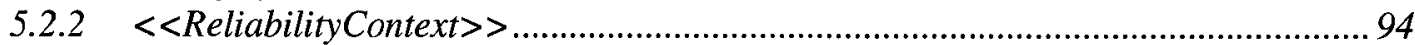

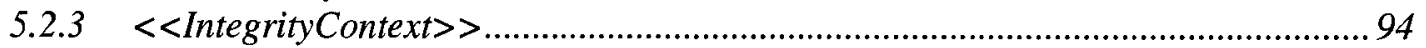




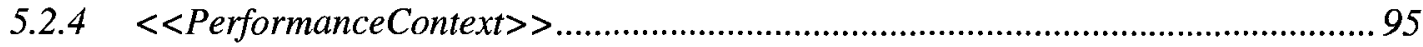

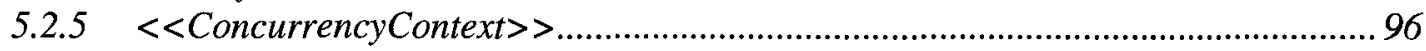

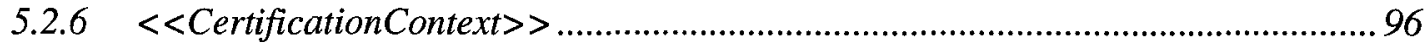

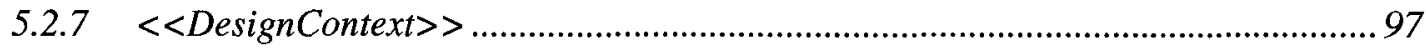

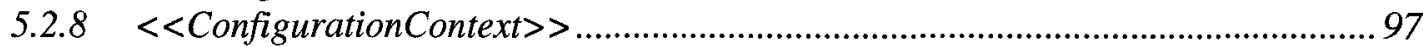

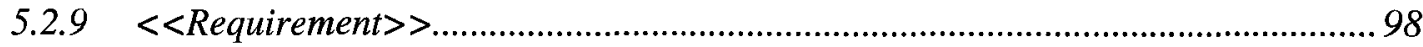

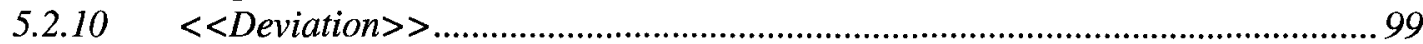

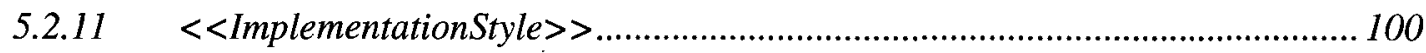

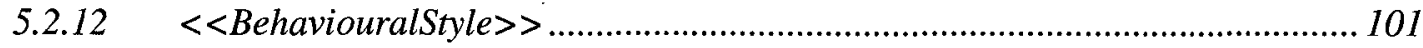

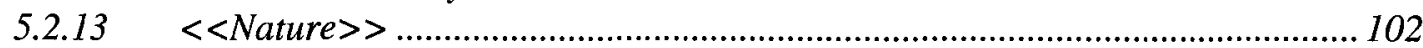

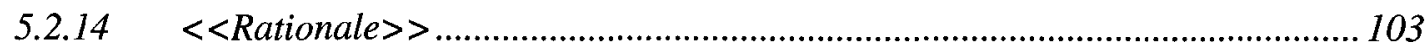

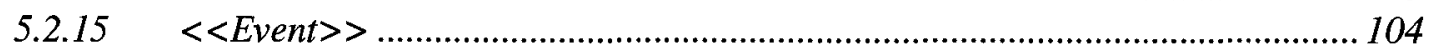

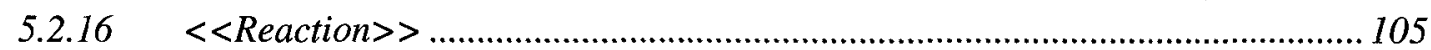

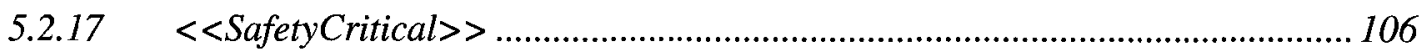

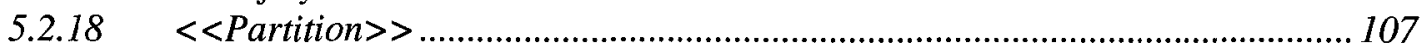

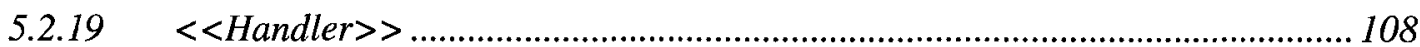

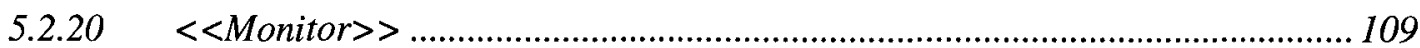

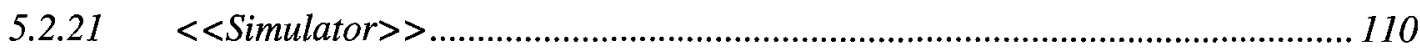

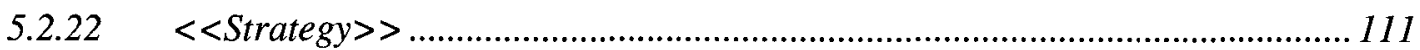

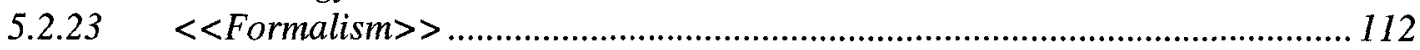

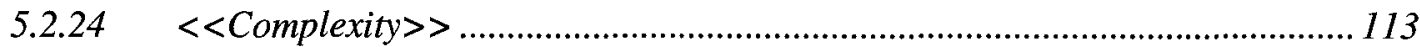

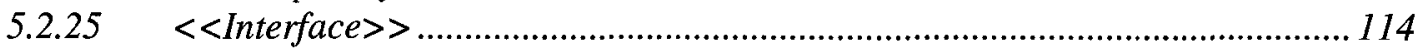

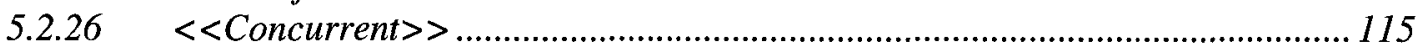

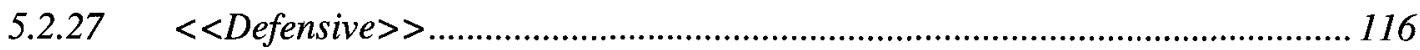

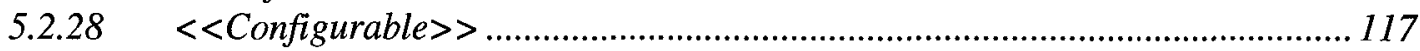

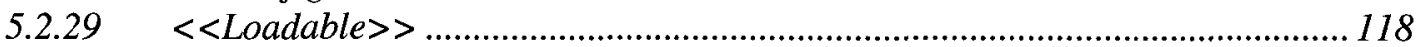

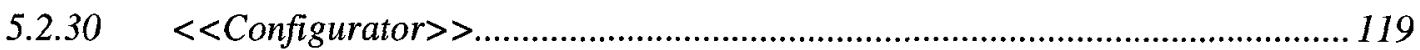

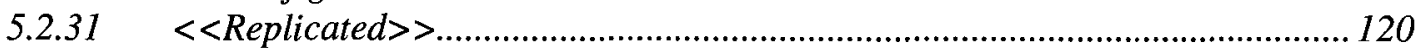

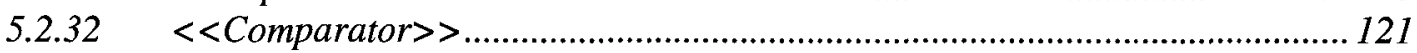

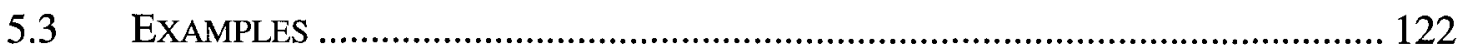

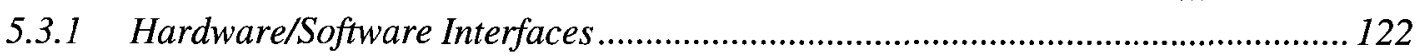

5.3.2 Contributions to Failure Conditions................................................................ 124

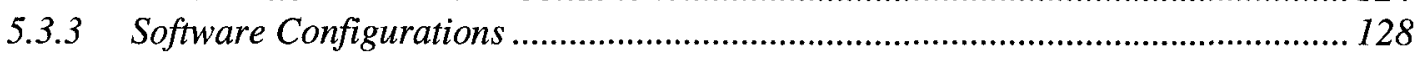

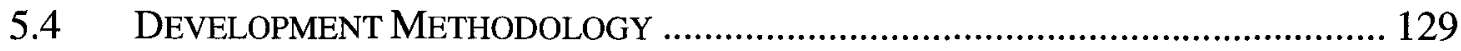

6 GENERATION OF CERTIFICATION INFORMATION ................................. 133

6.1 TECHNOLOGICAL REQUIREMENTS ........................................................... 133

6.1.1 Integrated Support in UML Modeling Tools.............................................. 134

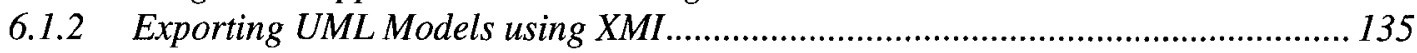

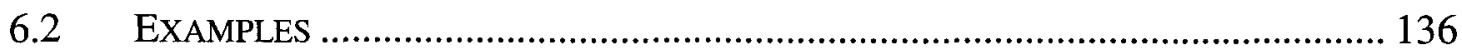

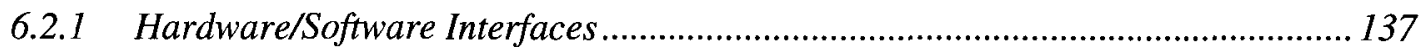

6.2.2 Contributions to Failure Conditions ................................................................. 138

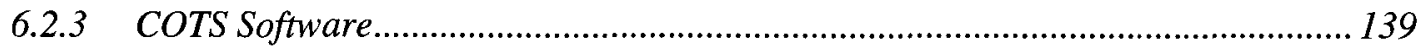

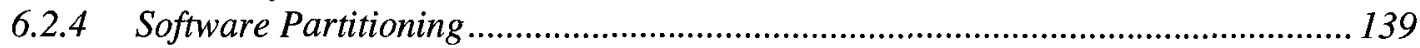

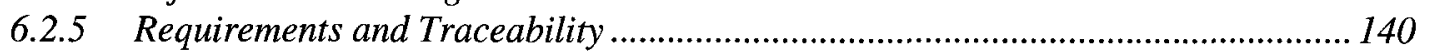

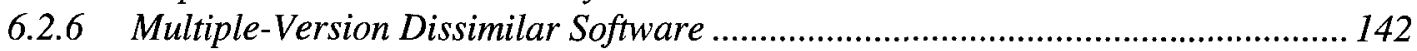

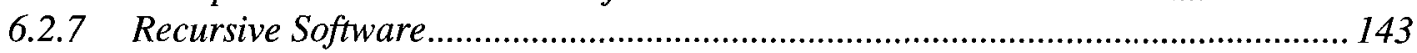

7 CASE STUDY - NAVIGATION CONTROLLER .............................................. 145 


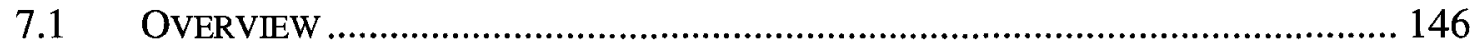

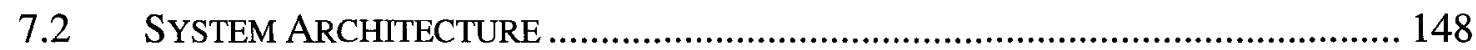

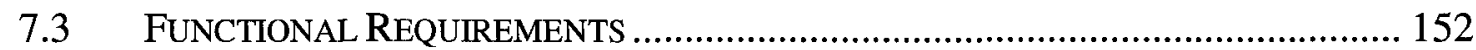

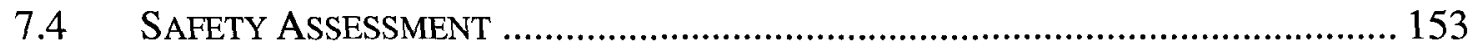

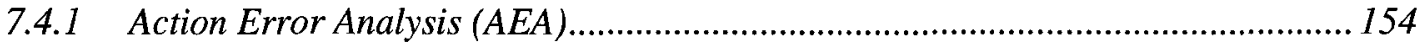

7.4.2 Failure Modes and Effects Analysis (FMEA) .................................................... 155

7.4.3 Hazards and Operability Analysis (HAZOP) ...................................................... 156

7.4.4 Interface Analyses (IA)................................................................................... 156

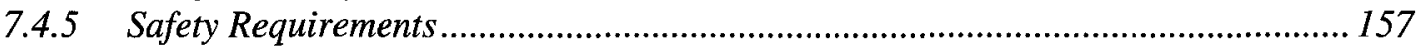

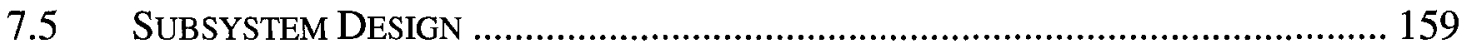

7.5.1 Identification of Events and Reactions................................................................ 160

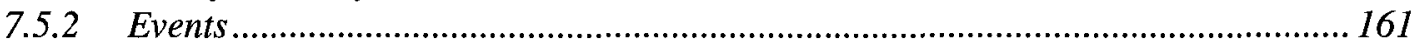

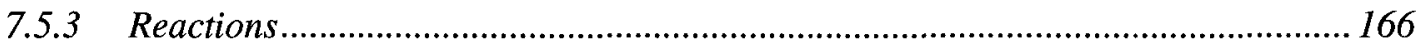

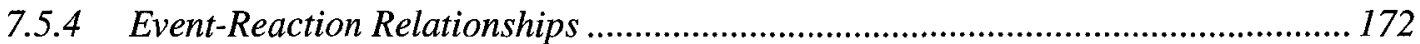

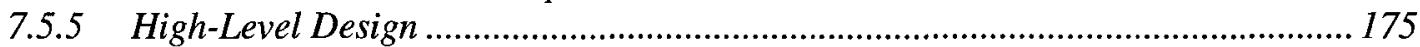

7.5.6 Low-Level Design of Events and Reactions .......................................................... 194

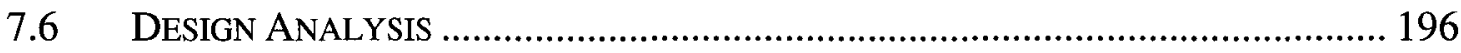

7.6.1 USAGE 1: Provide Safety Requirements ……........................................................ 196

7.6.2 USAGE 2: Design Safety Requirements in Systems ...........................................197

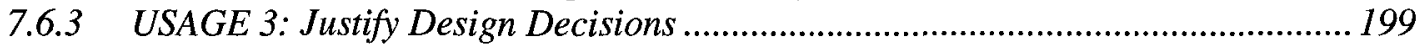

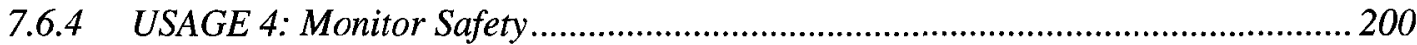

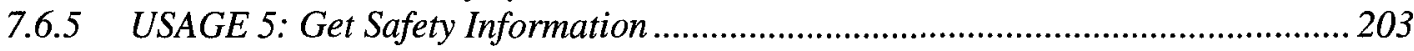

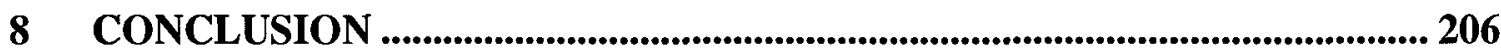

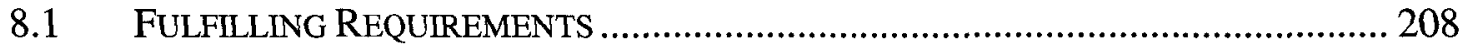

8.2 OPEN ISSUES AND FUTURE WORK........................................................ 210

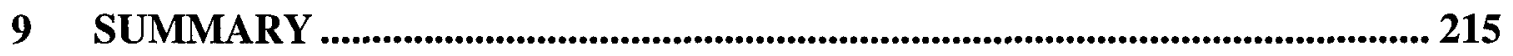

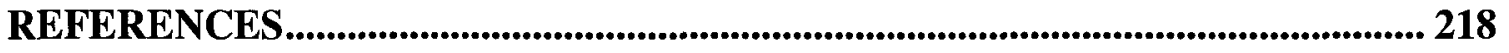

APPENDIX A EXAMPLES OF SAFETY/RISK ASSESSMENT METHODS 223

APPENDIX B EXAMPLES OF SAFETY-RELATED STANDARDS............. 226

\begin{tabular}{|c|c|}
\hline To & 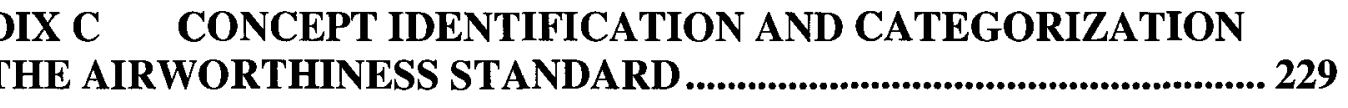 \\
\hline C.1 & 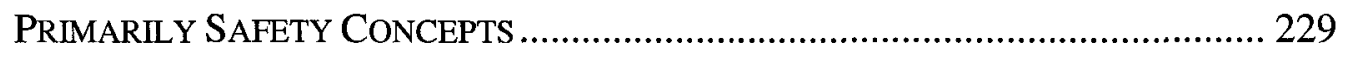 \\
\hline C. 2 & PRIMARILY RELIABILITY CONCEPTS ................ \\
\hline C. 3 & PRIMARILY INTEGRITY CONCEPTS ........................... \\
\hline C. 4 & PRIMARILY PERFORMANCE CONCEPTS \\
\hline C.5 & 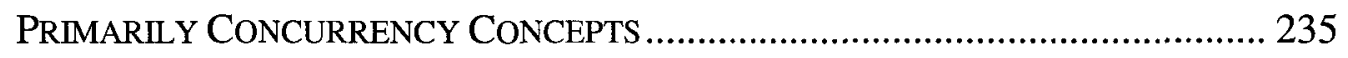 \\
\hline C. 6 & 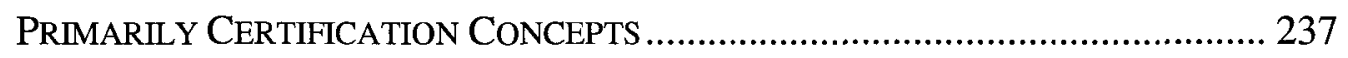 \\
\hline C.7 & 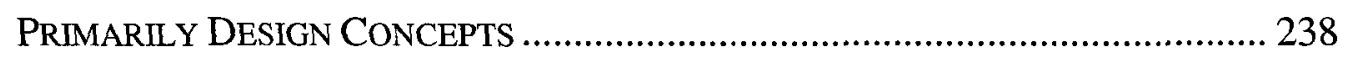 \\
\hline C.8 & ONFIGURATION CONCEPTS.................... \\
\hline
\end{tabular}


APPENDIX D CONCEPTUAL MODEL_CONCEPT RELATIONSHIPS ... 244

APPENDIX E GOMAA'S CLASS CLASSIFICATION ...................................... 250

APPENDIX F ASSESSING EXISTING PROFILES BASED ON THE INFORMATION REQUIREMENTS................................................................... 251

F.1 Quality OF SERVICE ANd FaUlt TOLERANCE OMG PROFILE (DISCUSSED IN

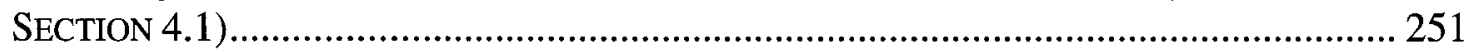

F.2 SCHEDUlabILITY, PERFORMANCE, AND TIME OMG PROFILE (DISCUSSED IN

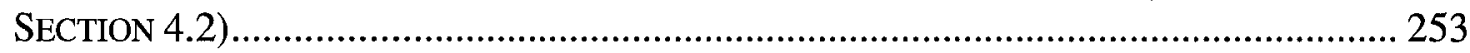

F.3 HIDOORS PROFILE (DISCUSSED IN SECTION 4.3) …………………............... 255

F.4 EFFECTS OF MESSAGE Loss, DELAY, AND CORRUPTION (DISCUSSED IN SECTION 4.4) 257

F.5 PATteRns For RELIABILITY AND SAFETy (DISCUSSED IN SECTION 4.5) .......... 259

F.6 AsSESSING EXISTING PROFILES BASED ON THE SAFETY INFORMATION REQUIREMENTS—SUMMARY (DISCUSSED IN SECTION 4.6) ........................................... 261

APPENDIX G ADDITIONAL UML PROFILE EXAMPLES ........................... 263

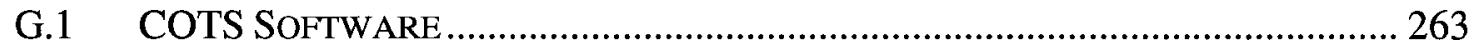

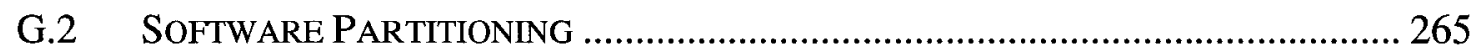

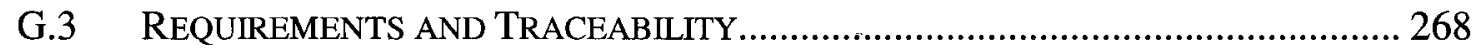

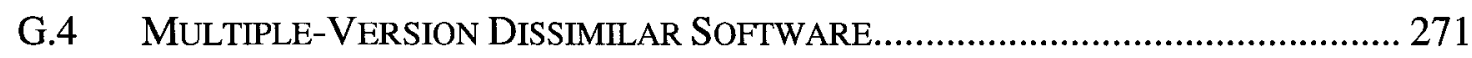

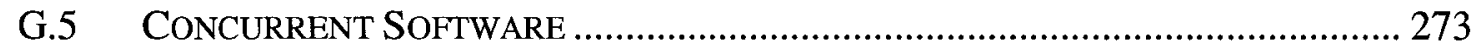

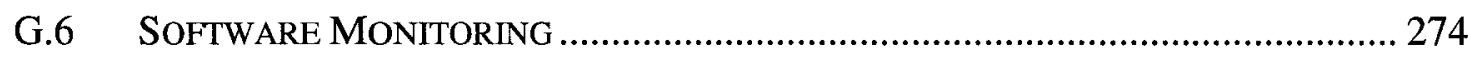




\section{LIST OF TABLES}

TABLE 1: DETAILS OF THE RESEARCH METHOD STEPS. 11

TABLE 2: EXAMPLES OF SAFETY REQUIREMENTS AND THE ACCIDENTS THEY PROTECT AGAINST. 13

TABLE 3: TRACING INFORMATION REQUIREMENTS TO THE ORIGINAL HIGH-LEVEL

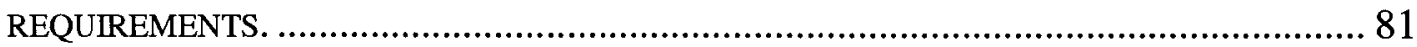

TABLE 4: DETAILS OF THE DEVELOPMENT METHODOLOGY STEPS. .................................. 132

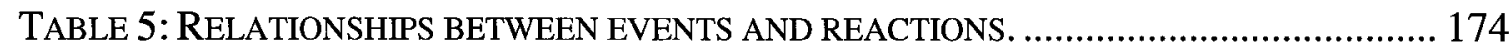

TABLE 6: USING THE PROPOSED UML PROFILE TO FULFILL THE INFORMATION REQUIREMENTS. 210

TABLE 7: EXAMPLES OF SAFETY OR RISK ASSESSMENT METHODS. 225

TABLE 8: SOME OF THE MANY SAFETY-RELATED STANDARDS THAT EXIST FOR SEVERAL INDUSTRIES. 228

TABLE 9: SAFETY-RELATED CONCEPTS THAT ARE CLASSIFIED AS “PRIMARILY SAFETY”. 231

TABLE 10: SAFETY-RELATED CONCEPTS THAT ARE CLASSIFIED AS "PRIMARILY RELIABILITY". 234

TABLE 11: SAFETY-RELATED CONCEPTS THAT ARE CLASSIFIED AS "PRIMARILY INTEGRITY". 234

TABLE 12: SAFETY-RELATED CONCEPTS THAT ARE CLASSIFIED AS "PRIMARILY PERFORMANCE". 235

TABLE 13: SAFETY-RELATED CONCEPTS THAT ARE CLASSIFIED AS "PRIMARILY CONCURRENCY". 236

TABLE 14: SAFETY-RELATED CONCEPTS THAT ARE CLASSIFIED AS "PRIMARILY CERTIFICATION". 238

TABLE 15: SAFETY-RELATED CONCEPTS THAT ARE CLASSIFIED AS “PRIMARILY DESIGN". 242

TABLE 16: SAFETY-RELATED CONCEPTS THAT ARE CLASSIFIED AS "PRIMARILY CONFIGURATION". 243

TABLE 17: ANALYSIS OF CONCEPTUAL MODEL CONCEPT RELATIONSHIPS. 249

TABle 18: Assessing the Quality OF SERVICE AND Fault Tolerance OMG PROFILE BASED ON THE INFORMATION REQUIREMENTS. 252

TABle 19: Assessing THE SChedUlabIlity, PeRformance, AND Time OMG PROFILE BASED ON THE INFORMATION REQUIREMENTS...................................................... 254

TABLE 20: ASSESSING THE HIDOORS PROFILE BASED ON THE INFORMATION REQUIREMENTS. 256

TABLE 21: ASSESSING THE EFFECTS OF MESSAGES PROFILE BASED ON THE INFORMATION

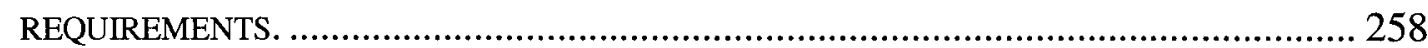

TABLE 22: ASSESSING PATTERNS FOR RELIABILITY AND SAFETY BASED ON THE INFORMATION REQUIREMENTS. ................................................................... 260

TABLE 23: ASSESSMENT SUMMARY OF EXISTING UML PROFILES BASED ON THE INFORMATION REQUIREMENTS. 262 


\section{LIST OF ILLUSTRATIONS (FIGURES)}

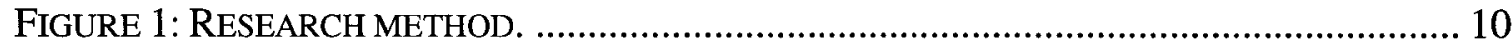

FIGURE 2: USAGE SCENARIOS FOR SAFETY INFORMATION....................................... 19

FIGURE 3: A UML MODEL SERVES AS A CENTRAL ROLE FOR STAKEHOLDERS. ................... 22

FIGURE 4: ROLE OF A UML SAFETY PROFILE IN THE DEVELOPMENT PROCESS. ................. 24

FIGURE 5: RELATIONSHIP BETWEEN SAFETY AND OTHER SAFETY-RELATED QUALITY CATEGORIES ASSUMING THAT THOSE CATEGORIES FORM MUTUALLY EXCLUSIVE SETS.

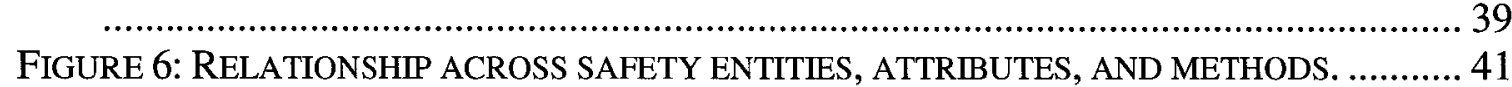

FIGURE 7: META-MODEL FOR THE REFINED SAFETY-RELATED CONCEPTS................45

FIGURE 8: KALMAN FILTER PROCESSING INPUT, THROUGH AN INTERFACE, FROM A

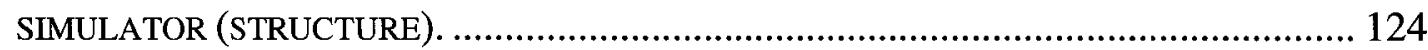

FIGURE 9: LANDING WHEEL CONTROLLER PROCESSING USER AND RADAR INPUTS

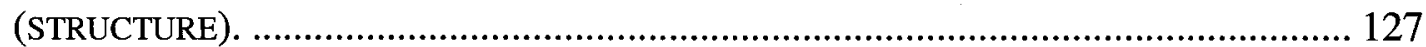

FIGURE 10: USER INTERFACE LANGUAGE CONFIGURATIONS (STRUCTURE). .................. 129

FIGURE 11: DEVELOPMENT METHODOLOGY OF AIRWORTHINESS-COMPLIANT SOFTWARE

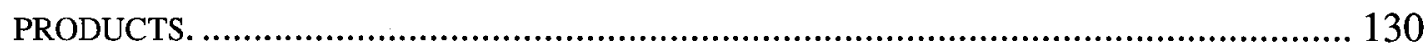

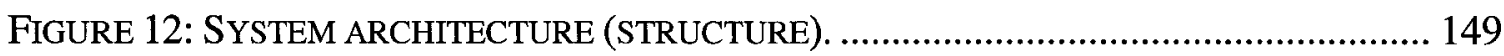

FIGURE 13: NAVIGATIONCONTROLLERSUBSYSTEM'S EVENTS (STRUCTURE).................. 162

FIGURE 14: NAVIGATIONCONTROLLER SUBSYSTEM REACTIONS (STRUCTURE)................ 167

FIGURE 15: NAVIGATIONCONTROLLER SUBSYSTEM'S HIGH-LEVEL DESIGN (STRUCTURE).

FIGURE 16: GOMAA'S CLASSIFICATION OF APPLICATION CLASSES USING STEREOTYPES [7]. 250

FIGURE 17: AIRCRAFT'S NAVIGATION CONTROLLER USING COTS SOFTWARE (STRUCTURE).

FIGURE 18: AIRCRAFT STEERING CONTROLLER (STRUCTURE) ...................................... 268

FIGURE 19: SAFETY-REQUIREMENTS FOR AN AIRCRAFT STEERING SYSTEM (STRUCTURE) 270

FIGURE 20: MULTIPLE-VERSION RADAR FILTER SYSTEM (STRUCTURE).......................... 272

FIGURE 21: CONCURRENT ACCESS TO AN AIRCRAFT'S COTS SOFTWARE (STRUCTURE). . 274

FIGURE 22: MONITORING THE AIRCRAFT'S ENGINE'S TEMPERATURE (STRUCTURE)........ 276 


\section{LIST OF ACRONYMS AND ABBREVIATIONS}

\begin{tabular}{|c|c|}
\hline AEA & Action Error Analysis \\
\hline AECL & Atomic Energy Canada, Limited \\
\hline AIAA & American Institute of Aeronautics and Astronautics \\
\hline ARINC & Aeronautical Radio, Incorporated \\
\hline $\mathrm{CCA}$ & Cause-Consequence Analysis \\
\hline CENELEC & European Committee for Electrotechnical Standardisation \\
\hline CORBA & Common Object Resource Broker Architecture \\
\hline COTS & Commercial-Off-The-Shelf \\
\hline DoD & United States Department of Defense \\
\hline EBNF & Extended Backus-Naur Form \\
\hline EMF & Eclipse Modeling Framework \\
\hline ESA & European Space Agency \\
\hline ETA & Event Tree Analysis \\
\hline FAA & Federal Aviation Administration \\
\hline FHA & Fault Hazard Analysis \\
\hline FIFO & First-In-First-Out \\
\hline FMEA & Failure Modes and Effects Analysis \\
\hline FMECA & Failure Modes, Effects, and Criticality Analysis \\
\hline FT & Fault-Tolerant / Fault Tolerance \\
\hline FTA & Fault Tree Analysis \\
\hline FTP & Fly-To-Point \\
\hline GPS & Global Positioning System \\
\hline HAZOP & Hazards and Operability Analysis \\
\hline HIDOORS & High Integrity Distributed Object-Oriented Real-Time Systems \\
\hline IA & Interface Analyses \\
\hline IEC & International Electrotechnical Commission \\
\hline IEE & Institution of Electrical Engineers \\
\hline IEEE & Institute of Electrical \& Electronic Engineers \\
\hline ISO & International Organization for Standardization \\
\hline LAT/LONG & Latitude and Longitude \\
\hline LED & Light-Emitting Diode \\
\hline
\end{tabular}




$\begin{array}{ll}\text { LIFO } & \text { Last-In-First-Out } \\ \text { LLF } & \text { Linear Logical Framework } \\ \text { MDA } & \text { Model-Driven Architecture } \\ \text { MISRA } & \text { Motor Industry Software Reliability Association } \\ \text { MoD } & \text { United Kingdom Ministry of Defence } \\ \text { MORT } & \text { Management Oversight and Risk Tree Analysis } \\ \text { MVC } & \text { Model-View-Controller } \\ \text { NASA } & \text { National Aeronautics and Space Administration } \\ \text { NATO } & \text { North Atlantic Treaty Organization } \\ \text { OCL } & \text { Object Constraint Language } \\ \text { OMG } & \text { Object Management Group } \\ \text { ORB } & \text { Object Resource Broker } \\ \text { PSAC } & \text { Plan for Software Aspects of Certification } \\ \text { QoS } & \text { Quality of Service } \\ \text { RF } & \text { Radio Frequency } \\ \text { RMA } & \text { Rate Monotonic Analysis } \\ \text { RTCA } & \text { Radio Technical Commission for Aeronautics } \\ \text { SAE } & \text { Society of Automotive Engineers } \\ \text { SIL } & \text { Safety Integrity Level } \\ \text { SMHA } & \text { State Machine Hazard Analysis } \\ \text { SPT } & \text { Schedulability, Performance, and Time } \\ \text { SQL } & \text { Structured Query Language } \\ \text { SSAC } & \text { Streamlining Software Aspects of Certification } \\ \text { SWOT } & \text { Strengths, Weaknesses, Opportunities, and Threats } \\ \text { VBA } & \text { Visual Basic for Applications } \\ \text { XMI } & \text { XML Metadata Interchange } \\ \text { XML } & \text { XSL }\end{array}$




\section{INTRODUCTION}

\subsection{Safety and UML}

Software's role in various systems has been rapidly increasing over past several decades. Its purpose is no longer restricted to managing financial or mathematical data. Due to the technological advances of computer processors, memory and other components, discrete hardware components in many systems have been replaced by software. Putting software on aircrafts, for example, has become significantly more affordable than it used to be. As a result, software now directly affects human life by managing flights, airplanes, ships, nuclear reactors, medical systems, and many others. This led to increased emphasis on the quality of software used in such systems. This emphasis focused on many aspects. First, it led to improved software verification and testing methods to detect software bugs before the software is delivered and deployed in its target system. However, it was accepted that software can never be $100 \%$ correct and error free. Therefore, fault tolerance emerged as a design technique to increase the reliability of the software. The principles of fault tolerance focus on adding protection mechanisms to detect software failures within a specified software boundary such that the software is able to recover and continue execution despite the presence of software faults or bugs. Therefore, fault tolerance aims at reducing the likelihood that the software becomes unavailable due to software bugs.

However, it was observed that highly reliable software is not necessarily safe within the context of the system in which it is used. Software is safe if it does not contribute to 
hazards within the context of the system in which it is used, and a system is safe if it does not cause accidents to or harm its environment. In particular, software may be reliable but unsafe when any of the following conditions occurs [1]:

1. The software correctly implements the requirements, but the specified behaviour is unsafe for the system as a whole (i.e. some requirements are unsafe).

2. Some safety-related requirements are missing (i.e. requirements are incomplete).

3. The software implements unintended and unsafe behaviour that is not specified in the requirements.

As a result, emphasis increased on developing safety requirements, whose goal is to ensure the safety of the environment in which the system is used. Safety requirements and constraints are generally the output of safety assessments that are performed on the system in which the software will be used. As a result, proper requirements development is vital towards ensuring safety.

Furthermore, safety-related standards generally require gathering information about the software that is not necessarily related to the implementation of safety-related requirements. Examples of such information include the use of COTS software and timerelated functions such as filters.

UML is the de-facto standard language for specifying, modeling, analyzing, and documenting software [2]. It is also used in other areas such as modeling systems, hardware, and even business contexts. UML represents a collection of best engineering techniques and practices that have proven successful in modeling large and complex 
software systems. It is a very important part of the software development process, and is particularly well-suited for developing object-oriented software. It uses mostly graphical notations to express the design of software systems. The benefits of UML include helping project teams communicate, explore potential designs, and validate the software architecture. It also increases the formalism of the software model, which makes the analysis process easier. Furthermore, it is the heart of the Model-Driven Architecture (MDA) initiative [3], whose supporters claim that it is the future of developing software.

UML is an extensible modeling language; it allows developers to add semantics to the UML language that are applicable in a particular domain, area, or industry. Such added semantics are called a "UML Profile", which in effect tailor the UML language to a specific area of interest such as, for example, fault tolerance, distributed computing, and Common Object Resource Broker Architecture (CORBA). A UML profile extends the core UML language by defining additional modeling mechanisms of the following types:

1. Stereotypes: A stereotype is used to describe a UML element in a platform or domain specific language.

2. Constraints: A constraint is a condition or a restriction that is applied to a UML element. It can be expressed in any language, regardless on whether it is machinereadable or not.

3. Tagged Value: A tagged value is used to further describe a stereotyped-element through parameterization of the stereotype in a platform or domain specific language. 


\subsection{Research Problem}

Safety assessments are performed on the system as a whole regardless of which of its features will be implemented in software. As a result, safety requirements are first developed for the system itself. Once it is determined which functionality will be implemented in software, the safety requirements associated with that functionality are allocated to the software that implements it.

Moreover, software certification authorities require information about the software that is not necessarily captured within the safety requirements. Such information could include the use of COTS software, time-related functions as filters, state machines, and others. The certification authorities consider this information along with the safety requirements when determining whether the software is safe or not.

Generally, safety engineers that perform the safety assessments and collect certification information are not the software engineers that design and implement the software. In fact, it is uncommon to find software engineers that are experienced with the safety and certification aspects of systems and software. Conversely, safety engineers are often inexperienced with software engineering's development techniques, including UML. This creates a critical gap that must be bridged - safety engineers need to have better insight into the software and to what extent it is compliant with the safety and certification requirements, and software engineers need to have better understanding of the safety and certification requirements so that they develop safe software that can run in a certified system. 
In this research, the airworthiness standard [4], which is the de-facto safety-related standard in the aerospace industry, is analyzed to extract a list of safety-related concepts that are of interest to both safety engineers and software engineers. It is argued that if those concepts are properly represented in UML models of software, then a tool can automatically generate reports containing safety and certification-related information about the software. This gives the safety engineers better insight into the software's safety and compliance aspects, which they can easily track over time. Those reports could also be used as evidence of software compliance with the airworthiness requirements, and then presented to the external certification authority. Furthermore, this will increase software engineers' knowledge of safety-related concepts, which will enable them to implement safer software and better communicate with safety engineers.

To model the safety-related concepts in UML, this research proposed a UML profile that can be used to model the safety-related information that is extracted from the airworthiness standard [4]. The proposed profile contains stereotypes and tagged values that correspond to the safety-related concepts, their attributes that capture the concept details, and the relationships among safety-related concepts. The focus here is modeling safety and certification information in structural diagrams, specifically class diagrams, but the stereotypes and tagged values should be easily transferable to dynamic diagrams such as object diagrams and state charts.

\subsection{Document Organization}

Section 1.4 of this document describes the research method that was followed in this research. 
Section 2 describes the industrial view of this research. Section 2.1 describes safety assessments in general, and then provides examples of safety requirements and safety assessment techniques. Section 2.2 lists several safety-related industrial standards and then provides a high-level description of the Radio Technical Commission for Aeronautics (RTCA) DO-178B [4] airworthiness standard. Section 2.3 presents research findings on the challenges of developing safety-critical software that has certification requirements. Section 2.4 presents and describes usage scenarios for safety information, and proposes using UML to model the safety information. Section 2.5 identifies traceability between software concepts that needs to be tracked. Section 2.6 discusses the rationale, disadvantages, advantages, and requirements of using a UML profile to model safety information.

Section 3 introduces safety-related concepts that are extracted from the airworthiness standard, RTCA DO-178B [4]. Section 3.1 identifies, describes, and categorizes the safety-related concepts as extracted from the airworthiness standard. Each category is prefixed with "primarily" to indicate that its concepts are related to other categories as well. The concepts are then refined in section 3.2. Section 3.2.1 introduces the conceptual model describing the refined concepts. Section 3.2.2 describes each concept in detail, presents their attributes and relationships with other concept, and explains which original safety-related concept from section 3.1 can be represented using each of the refined concepts. Section 3.2.4 explains how the refined concepts, and their conceptual model, satisfy the required traceability explained in section 2.5 . Section 3.3 identifies precise information requirements that a suitable UML profile should be able to model. 
Section 4 presents some of the existing UML profiles and patterns and assesses each one of them versus the information requirements identified in section 3.3. Section 4.1 introduces the "UML Profile for Modeling Quality of Service and Fault Tolerance Characteristics and Mechanism" [5], which is an Object Management Group's (OMG) UML profile that enables modeling some safety and fault tolerance concepts. Section 4.2 introduces the "UML Profile for Schedulability, Performance, and Time Specification" [6], which enables modeling some concepts that are usually important in safety-critical software such as performance and concurrency. Section 4.3 introduces a UML profile that was developed by a European research project that specialized in developing highintegrity real-time systems [26]. Section 4.4 introduces a UML profile that was developed by a researcher who argued that safety is often related to messages across software components [27]. Section 4.5 introduces patterns that can be used to model some reliability and safety concepts such as software redundancy. Section 4.6 summarizes the overall suitability of each profile with respect to the information requirements identified in section 3.3.

Section 5 presents a new UML Profile, one for modeling safety-related concepts listed in section 3.2, and provides examples of its usage. Section 5.1 presents the UML profile, section 5.3 provides numerous examples of its usage, and section 5.4 describes a development methodology for safety-critical systems within which the proposed UML profile can be used.

Section 6 describes how safety certification information can be generated from a UML model where the new UML profile is applied. Section 6.1 describes the technological requirements that are needed to be able to generate such safety and certification 
information, with sections 6.1.1 and 6.1.2 providing guidance on how such requirements can be achieved. Section 6.1.1 describes how UML modeling tools can be extended to support generation of certification information, an section 6.1.2 introduces another approach that uses XML Metadata Interchange (XMI) to obtain the safety and certification information. Section 6.2 presentes examples of search queries that can be executed on a UML model to generate safety and certification information from the software model.

Section 7 presents a case study of an aircraft's navigation controller subsystem, which controls the movement of an aircraft by performing autopilot and custom Fly-To-Point (FTP) positions from the pilots. Section 7.1 provides an overview of the system and presents some navigation terminology. Section 7.2 describes the aircraft's system architecture in which the navigation controller subsystem appears. Section 7.3 lists the functional requirements of the navigation controller subsystem. Section 7.4 discussed the safety assessment that was performed on the system and lists the identified safety hazards that are relevant to the subsystem under study. Section 7.4.1 lists safety hazards that were identified using the Action Error Analysis (AEA) safety assessment method, section 7.4.2 lists those that were identified using the Failure Modes and Effects Analysis (FMEA) method, section 7.4.3 lists those that were identified using the Hazards and Operability Analysis (HAZOP) method, and section 7.4.4 lists those that were identified using the Interface Analyses (IA) method. Section 7.4.5 lists safety requirements that were assigned to the navigation controller subsystem based on the hazards identified in sections 7.4.1 - 7.4.4. Section 7.5 presents the UML model for the subsystem design using the proposed UML profile, with section 7.5.2 showing the subsytem's safety- 
related events of interest, section 7.5.3 showing the subsystem's reactions to those events, section 7.5.4 explicitly listing all the relationships between events and reactions, section 7.5.5 showing the subsystem's high-level design, and section 7.5.6 presenting approaches to low-level design of events and reactions. Section 7.6 discusses the benefits of the proposed UML profile by analysis the UML model of the subsystem according to the usage scenarios identified in section 2.4 .

Section 8 concludes this research by describing the use of the proposed UML profile and identifying open issues for future work. Section 8.1 assesses the UML profile according to the requirements identified in section 2.6.3 the same way existing UML profiles were assessed in section 4. Section 8.2 lists open issues and improvement opportunities for future work.

Section 9 provides a summary of this document.

\subsection{Research Method}

This research was performed according to the method described in Figure 1. There is no input or entry criterion for the first step, S-1. The input of each of the other steps is all the outputs from all of its previous steps. The entry criterion of those steps is the exit criterion of its previous step. Notice, however, that the input to step S-2 includes the outputs of both steps S-1 and S-7 when it is entered from step S-7. The exit criterion for each step is that its output becomes available. The activity, output, and reference sections of each step are presented in Table 1 below. 


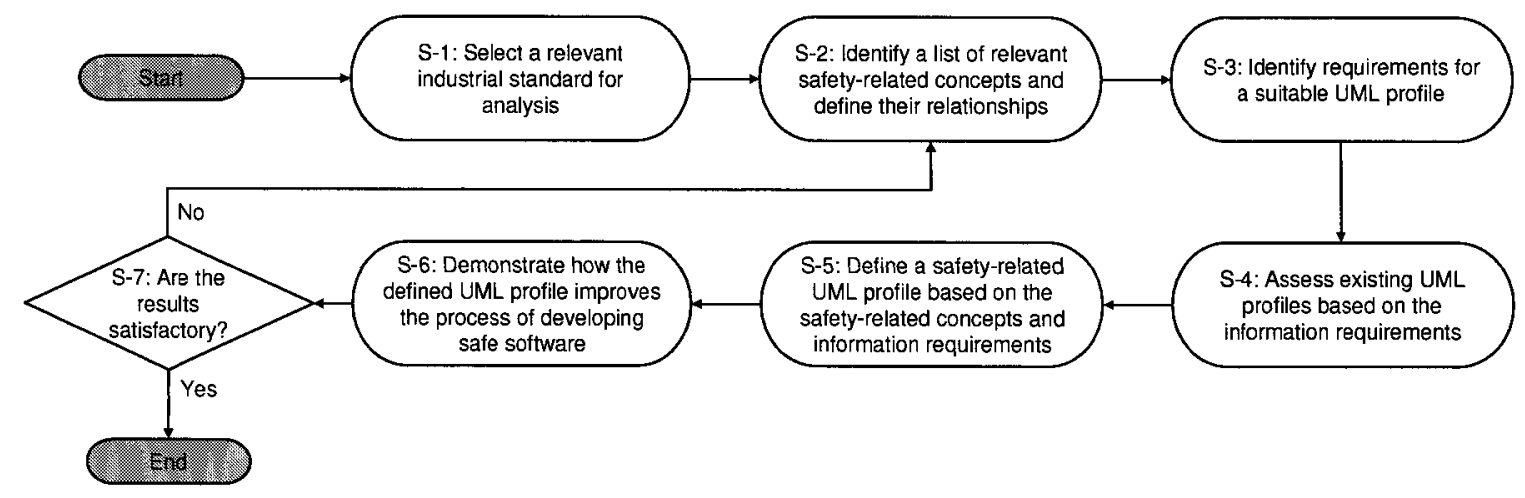

Figure 1: Research method.

\begin{tabular}{|l|l|}
\hline Step & \multicolumn{1}{|c|}{ Description } \\
\hline S-1 & $\begin{array}{l}\text { Select a relevant industrial standard for analysis } \\
\text { Activity: A safety-related standard is identified for analysis. } \\
\text { Output: A list of one or more standards that are selected for further analysis. } \\
\text { Reference: Section 2.2. }\end{array}$ \\
\hline S-2 & $\begin{array}{l}\text { Identify a list of relevant safety-related concepts and define their relationships } \\
\text { Activity: Safety-related concepts that are emphasized in the standard selected in S- } \\
\text { friendlier from a software modeling perspective. Relationships across the refined } \\
\text { safety-related concepts are defined through a conceptual model. } \\
\text { Output: A detailed list of safety-related concepts and their definitions, which } \\
\text { includes the definition of each concept as it is used in the selected standard, and a } \\
\text { list of refined safety-related concepts including a conceptual model describing their } \\
\text { inter-concept relationships. } \\
\text { Reference: Sections 3.1 and 3.2. }\end{array}$ \\
\hline S-3 & $\begin{array}{l}\text { Identify requirements for a suitable UML profile } \\
\text { Activity: The safety-related concepts identified in S-2 are further analyzed. } \\
\text { Requirements for developing software under the identified standard, as well as } \\
\text { software-related requirements for certifying systems, are understood. Information } \\
\text { that a suitable UML profile should be able to model are developed into information } \\
\text { requirements. } \\
\text { Output: A list of high-level requirement, and a list of information requirements } \\
\text { specifying which information a suitable UML profile should be able to model. } \\
\text { Reference: Sections 2.6.3 and 3.3. }\end{array}$ \\
\hline
\end{tabular}




\begin{tabular}{|c|c|}
\hline Step & \\
\hline S-4 & $\begin{array}{l}\text { Assess existing UML profiles based on the information requirements } \\
\text { Activity: Identify existing UML profiles that are related to the development of } \\
\text { safety-critical software. Assess each one of them based on the information } \\
\text { requirements identified in S-3. } \\
\text { Output: An assessment of existing UML profiles and how they perform versus the } \\
\text { identified information requirements. } \\
\text { Reference: Section } 4 \text {. }\end{array}$ \\
\hline$S-5$ & $\begin{array}{l}\text { Define a safety-related UML profile based on the safety-related concepts and } \\
\text { information requirements } \\
\text { Activity: A safety-related UML profile that fulfils the information requirements is } \\
\text { defined. This UML profile is able to represent the refined safety-related concepts } \\
\text { on UML designs, thus enabling engineers to better meet the challenges and } \\
\text { requirements identified in S-2. A system and software development methodology } \\
\text { for using the UML profile is presented. } \\
\text { Output: A safety-related UML profile, examples of its usage, and a development } \\
\text { methodology for using the UML profile. } \\
\text { Reference: Section 5. }\end{array}$ \\
\hline S-6 & $\begin{array}{l}\text { Demonstrate how the defined UML profile improves the process of developing safe } \\
\text { software } \\
\text { Activity: The degree to which a software model using this profile describes the } \\
\text { safety and certification aspects of the system is considered. An analysis of how the } \\
\text { requirements in section } 3.3 \text { are fulfilled is presented. Approaches are proposed on } \\
\text { how a UML modeling tool can be used to extract certification information from a } \\
\text { UML model using this profile are presented. A case study using the profile is } \\
\text { performed. } \\
\text { Output: A proposed approach on how a UML modeling tool can extract safety and } \\
\text { certification-related information from a UML model using this profile, an analysis } \\
\text { of the profile versus the information requirements identified in section } 3.3 \text {, and a } \\
\text { case study using the profile. } \\
\text { Reference: Sections } 6,7 \text {, and } 8.1 \text {. }\end{array}$ \\
\hline S-7 & $\begin{array}{l}\text { Are the results satisfactory? } \\
\text { Activity: The results of steps S-5 and S-6 are assessed. If they satisfactorily } \\
\text { improve the development process of safety-critical software, then the process is } \\
\text { complete. If not, then step S-2 is revisited for another iteration of this process. } \\
\text { Output: The decision on whether to perform another iteration of this process, } \\
\text { starting with step S-2, the strengths of the defined UML profile, and a list of open } \\
\text { issues in this work. } \\
\text { Reference: Section } 8 \text {. }\end{array}$ \\
\hline
\end{tabular}

Table 1: Details of the research method steps. 


\section{INDUSTRIAL PRESPECTIVE}

\subsection{Safety Assessments}

Safety-critical software must exhibit safe behaviour that does not contribute to hazards within the context in which it is used. For example, an aircraft must only allow the pilot to hide the landing wheel if it is flying in the air. If the landing wheel was hidden while the aircraft is on ground, then there would be a hazard of damaging the aircraft and hurting its occupants. A hazard is a state of the system that could ultimately lead to an accident that may result in a loss in human life.

Because of such added requirements and constraints, developing safety-critical software is more expensive than developing non-safety-critical software. In fact, it is generally well accepted that developing safety-critical software is at least 10 times more expensive than non-safety-critical software, and some sources claim that it can be 20 to 30 times more expensive [8].

Many standards require that a safety assessment be performed for each safety-critical system. Safety assessments, which have some similarities with risk assessments [1] and are performed using similar methods, produce a list of safety requirements and constraints that the system developers must adhere to. Performing a safety assessment is a mandatory and critical element to developing a safety-critical system. Table 2 provides examples of safety requirements and the potential accidents they protect against. 


\begin{tabular}{|l|l|}
\hline \multicolumn{1}{|c|}{ Safety Requirement } & \multicolumn{1}{|c|}{ Accident Protected Against } \\
\hline $\begin{array}{l}\text { A submarine detection aircraft may only } \\
\text { release a sonobuoy while it is flying above } \\
\text { water }\end{array}$ & $\begin{array}{l}\text { The sonobuoy is dropped on unintended } \\
\text { locations and causes unintended damages }\end{array}$ \\
\hline $\begin{array}{l}\text { An aircraft's automatic flight pilot programme } \\
\text { may only fly the aircraft to a particular } \\
\text { destination after explicit confirmation from the } \\
\text { pilot }\end{array}$ & $\begin{array}{l}\text { The automatic flight pilot programme flies the } \\
\text { through hazardous flight paths }\end{array}$ \\
\hline
\end{tabular}

Table 2: Examples of safety requirements and the accidents they protect against.

There exist many methods for performing a safety or risk assessment. Those methods differ, for instance, in terms of what factors they consider as possible causes of accidents (e.g., operator actions, environment state), the subjects they analyze (e.g., critical events, possible failures), their outputs (e.g., a tree), their scalability. Furthermore, some methods target reliability issues (e.g., Failure Modes and Effects Analysis (FMEA), Failure Modes, Effects, and Criticality Analysis (FMECA), Interface Analyses (IA)) whereas others solely consider safety issues (e.g., State Machine Hazard Analysis (SMHA)), and some consider both (e.g., Event Tree Analysis (ETA)). Most of those methods originated in hardware or system analysis, and some were developed for software. Some consider single events at a time (e.g., FMEA and FMECA), whereas others consider relationships across events (e.g., Fault Tree Analysis (FTA)). Furthermore, some are used to identify potential hazards (e.g., Action Error Analysis (AEA)), whereas others are used to analyze previously identified hazards (e.g., ETA).

Therefore, some of these methods are complimentary to each other, whereas others are similar and overlap. As a result, a project is likely to use several of those methods rather 
than just one. Examples of those methods are listed in Table 7 of Appendix A. Those methods are discussed in many references, and a summary of them is presented in [1].

\subsection{Safety-Related Standards}

Many industrial standards exist for system and software safety. Some are common to all industry sectors (e.g., IEC 61508-3 on software requirements for the functional safety of electrical/electronic/programmable electronic systems) whereas others are industry specific (e.g., CENELEC 50128 for Railway applications). Table 8 of Appendix B lists some of these standards that relate to safety, or reliability due to its relation to safety. Hermann provided a high-level summary for those standards, or some of their earlier versions, in [9].

RTCA DO-178B [4] is the de-facto safety-related standard for developing software to run in aerospace systems. It is also known as the "airworthiness" standard. Consequently, engineers whose responsibilities includes ensuring compliancy with the airworthiness standard are known as "airworthiness engineers".

In addition to developing safety-related requirements, standards usually have additional certification-related requirements. Those requirements are not necessarily implemented in software, but rather they represent information about the software that must be submitted to the certification authorities. For example, the airworthiness standard requires that developers submit information regarding the COTS software used in the system. Moreover, it requires that the developers specify time-related functions, such as filters, that are used in the system. Therefore, it is important to be able to gather such information easily about the software. 
DO-178B realizes that not all software components in an airborne system have the same impact on the safety of the aircraft and its occupants. For example, the failure of software that controls the altitude of an aircraft is much less acceptable than the failure of the software that controls the aircraft VCR for watching movies. This is because the failure of the former may significantly reduce the aircraft's chances of a safe flight. The failure of the latter, however, does not have such effects as long as the VCR is isolated from other safety-critical software. As a result, DO-178B classifies software failure conditions into the following five categories [4]:

1. Catastrophic: A failure condition of this type would prevent continued safe flight and landing of the aircraft. A software component whose failure may result in failure condition of this category is known as a level A software component, and is said to have airworthiness level A.

2. Hazardous/Severe-Major: A failure condition of this type would introduce operating conditions that would severely reduce the ability of the aircraft crew to cope with them to the extent where there would be large reductions in system safety, the inability of the crew to perform tasks accurately and completely, and potential fatal injuries. A software component whose failure may result in failure condition of this category is known as a level B software component, and is said to have airworthiness level B.

3. Major: A failure condition of this type would introduce operating conditions that would severely reduce the ability of the aircraft crew to cope with them to the extent where there would be significant reductions in system safety and crew 
efficiency, significant increase in crew workload and occupant discomfort, and potential injuries. A software component whose failure may result in failure condition of this category is known as a level C software component, and is said to have airworthiness level C.

4. Minor: A failure condition of this type would introduce operating conditions that can be handled by the crew, but may include a slight increase in the crew's workload or occupant discomfort, and a slight reduction in safety. A software component whose failure may result in failure condition of this category is known as a level D software component, and is said to have airworthiness level D.

5. No Effect: A failure condition of this type does not impact the system safety of the aircraft, nor does it increase the aircraft crew's workload. A software component whose failure may result in failure condition of this category is known as a level $\mathrm{E}$ software component, and is said to have airworthiness level E.

It should be noted, however, that there exists a difference between the concepts of "airworthiness" and "safety". The airworthiness standard, like many other safety-related standards, defines different levels of impact on safety called "failure condition categories" and "software levels". However, it defines the failure condition categories and the software levels based on the "severity of failure conditions on the aircraft and its occupants" [4]. This is different from Leveson's definition of safety, which was stated as "the freedom from accidents or losses" [1]. While safety is the freedom from accidents or losses, airworthiness is therefore the freedom from accidents or losses with respect to the aircraft and its occupants. Thus, airworthiness is a subset of safety, and safe software is 
airworthy but airworthy software is not necessarily safe. For example, the first safety requirement in Table 2 of page 13 is not necessarily an airworthiness requirement, whereas the second one is.

\subsection{Challenges in Software Safety}

The NASA Langley Research Center, which has long cooperated with the Federal Aviation Administration (FAA) on research about software engineering methods for aerospace applications, conducted a research programme called Streamlining Software Aspects of Certification (SSAC). This programme included an extensive survey to identify the challenges in developing safety-critical software for aerospace systems. Hayhurst and Holloway have documented results of this research in [10].

Hayhurst and Holloway of the NASA Langley Research Center identified "the challenge of accurately communicating requirements between groups of people" as "the root of many of the current challenges" in software safety [10]. They presented the communication challenge as a combination of the following two major communication channels:

1. Between regulatory people (e.g. certification authorities) and systems people (e.g. systems engineers and airworthiness engineers).

2. Between systems people (e.g. systems engineers and airworthiness engineers) and software people (e.g. software engineers).

Since systems engineers and safety/airworthiness engineers need to communicate with the certification authorities, they need to have insight into the software and its sfety 
compliance aspects. The fact that they are unlikely to be experienced in software engineering makes their responsibilities even more challenging. Moreover, software is continuously changing and it is likely that the software engineers significantly outnumber safety engineers. Therefore, it is essential to be able to achieve insight into the software's compliance aspects at relatively low costs. Such insight could be the ability to easily monitor the software engineers' progress with respect to the safety requirements and the compliance with the certification requirements of the software.

Hayhurst's and Holloway's survey also found out that "requirements definition is difficult" [10]. This undoubtedly contributes to the communication challenges between the various people groups. For example, systems engineers may define requirements that software engineers find unusual or expensive. If software engineers better understand the needs behind the requirements, then they may be able to propose solutions that are more cost effective. The software engineers' misinterpretation of the requirements may also be due to their lack of experience in safety. In fact, their lack of experience in safety often causes them to confuse safety with reliability. In many instances, software engineers cannot clearly define software safety without having prior background knowledge or experience.

\subsection{Usage Scenarios for Safety Information}

Based on the discussion in sections $2.1-2.3$, safety information is used by many stakeholders as described in the use case diagram in Figure 2. 


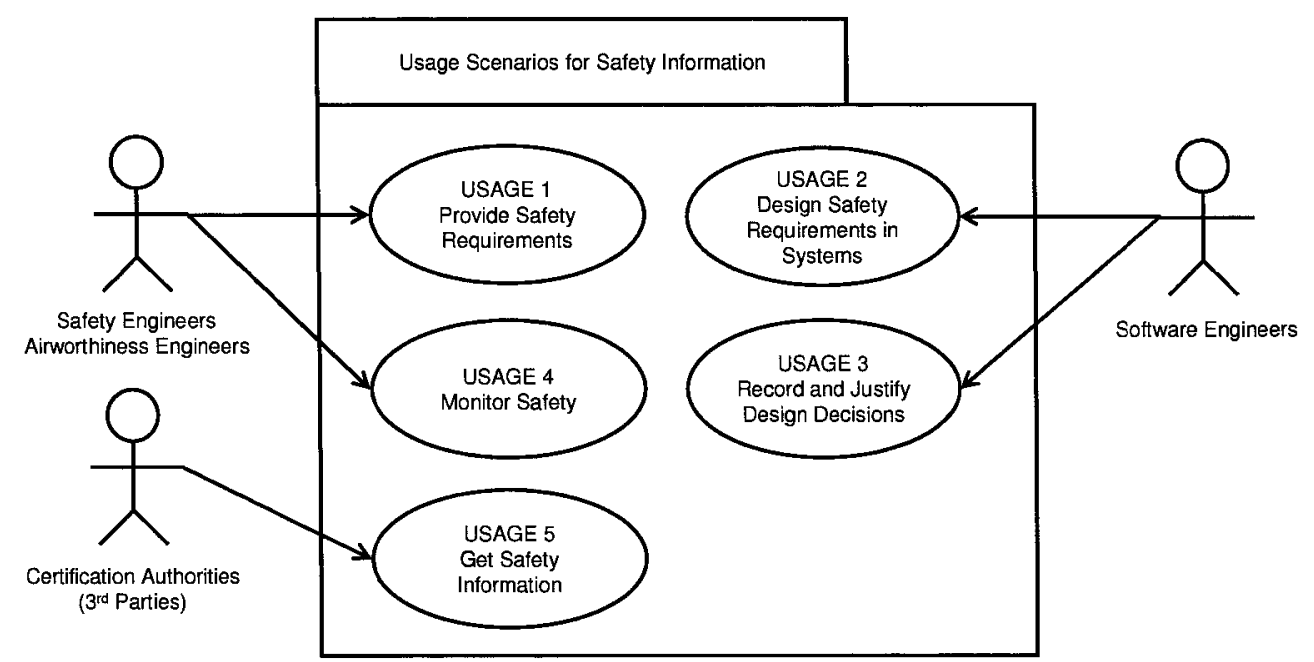

Figure 2: Usage scenarios for safety information.

The usage scenarios are:

USAGE 1 Provide Safety Requirements: Safety and airworthiness engineers perform a safety assessment of the system being designed or modified. As discussed in section 2.1, such a safety assessment results in safety requirements, a subset of which will be allocated to software and communicated to the software engineers. The software engineers will then design and implement the software according to the safety information and requirements. Thus, this usage scenario represents the process of communicating safety information from the safety and airworthiness engineers to the software engineers.

USAGE 2 Design Safety Requirements in Systems: Once the software engineers are informed with the safety requirements allocated to software, they design the software system with the safety requirements in mind. Then, they implement it such that it meets all the safety requirements. Thus, this 
usage scenario represents the process of designing and implementing the software system according to the safety requirements.

USAGE 3 Justify Design Decisions: Not only must the software engineers design the software to meet the safety requirements, but they must justify their design decisions as well. Such justification should explain the rationale for the architecture and design details. In practice, architectural and major design decisions are documented in separate documents, which makes it separate from the software model. Furthermore, detailed design decisions normally appear as plain text comments in the source code, which makes it hard for safety and airworthiness engineers to obtain justifications for the various design decisions. Thus, this usage scenario represents the process of justifying and documenting design decisions so that they can be easily obtained in the future.

USAGE 4 Monitor Safety: The safety and airworthiness engineers continuously monitor the safety of the system, including the software, over the project's lifecycle. In order to do so, they need to consider how the software engineers designed the software (USAGE 2) according to the safety requirements they were provided with (USAGE 1). The software engineers' justifications for the design decisions (USAGE 3) will also be considered. Then, the safety and airworthiness engineers can assess this information and discuss any issue with the software engineers. This ensures that the software's safety is continuously improving during the software's lifecycle so that it meets the final safety objectives of the 
system. In addition, this usage scenario provides additional confidence that the system certification process will go more smoothly. Thus, this usage scenario represents the process of continuously monitoring the design and implementation of the software in accordance with the system's overall safety requirements.

USAGE 5 Get Safety Information: Once it is time to certify the system, which is usually towards the end of the development lifecycle, safety and certification information is submitted to the certification authorities. This information includes the safety requirements (USAGE 1), the software design (USAGE 2), the justification of the software design (USAGE 3) given the safety requirements of the software, and the process used to continuously monitor the system and software safety over the development lifecycle (USAGE 4). If safety and airworthiness engineers continuously and appropriately monitor safety over time (USAGE 4), then certification should be a much easier experience. Thus, this usage scenario represents the process of obtaining the appropriate safety information, system and software design, justification of the design from a safety perspective, and the method used for monitoring safety during the development lifecycle for the purpose of submitting this information to the certificatrion authorities.

If the safety information is captured in a UML model, then this would easily facilitate the above mentioned usage scenarios. UML models are developed for software systems anyways, so using it to facilitate the usage scenarios and address the challenges in 
software safety fits well (this is rationalized further in section 2.6). Therefore, a UML model can serve a central role as shown in Figure 3.

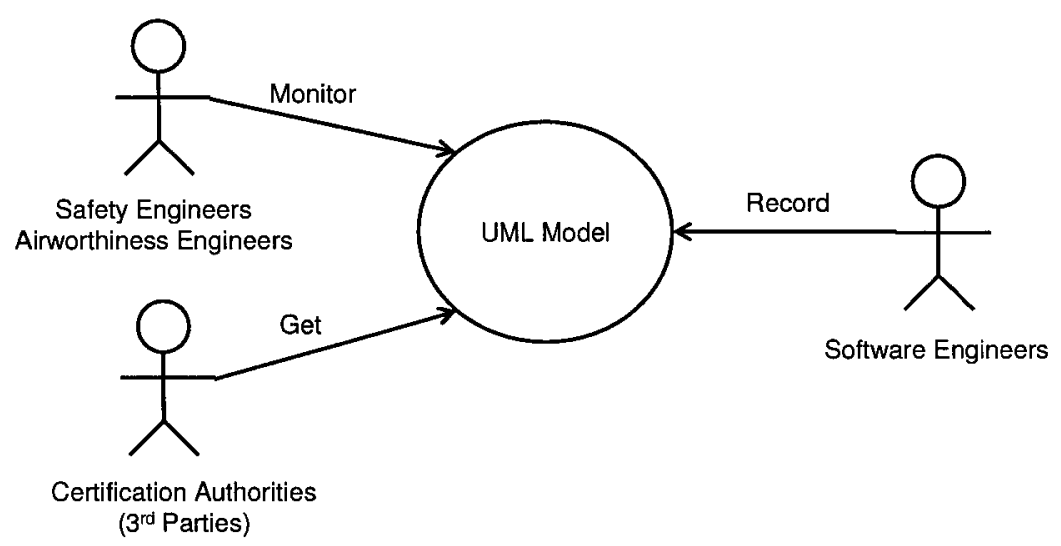

Figure 3: A UML model serves as a central role for stakeholders.

As Figure 3 shows, a UML model can serve as a central role and a key element in the communication of safety information across engineering groups. Software engineers record safety information in UML models. Then, safety and airworthiness engineers can monitor the safety information by automatically generating reports about it, using a tool, from the UML model. Therefore, they need not understand the UML model because any tool that extracts the safety information from the model can format it in a modelindependent way. When it is time to certify the system, certification authorities can get the safety and certification information from the UML model, again, using a tool which could produce the safety and certification information in a format that is suitable for submission to the certification authorities.

\subsection{Traceability Requirements}

Proper traceability is key in the development of large systems, and it is even more important for the development of safety-critical systems. For example, the airworthiness 
DO-178B standard [4] requires traceability across the development lifecycle. In fact, it requires that at least the software design be traceable to the original high-level requirements for all software of level $\mathrm{D}$ or higher. Therefore, it is important to be able to trace design elements to the requirements.

Another important traceability requirement relates to the analysis of safety requirements. For example, the first requirement in Table 2 states: A submarine detection aircraft may only release a sonobuoy while it is flying above water. This can be rephrased to: The ordnance (sonobuoy release) subsystem shall not release a sonobuoy if it is requested to do so when the aircraft is not flying above water. This results in identifying the following event of interest: The ordnance subsystem is requested to release a sonobuoy when the aircraft is not flying above water. Furthermore, this also results in identifying a reaction to this event, namely: The ordnance subsystem verifies that the aircraft is flying above water, and forbids the sonobuoy from being released if it is not flying above water. Since reactions are caused by events, it is important to ensure that every reaction is traceable to the appropriate events

In summary, two important traceability requirements are:

1. Tracing software design elements to software requirements.

2. Tracing software reactions to software events.

\subsection{UML APPROACH}

Because the results of safety assessments are an important part of software developers' work, they need to be appropriately communicated and implemented in the technical 
designs. Furthermore, certification information about the software is required for the certification authorities. It is appropriate and useful to be able to represent the results of safety assessments and certification information in UML diagrams because it is the standard modeling language used by software developers throughout the world [2]. Currently, there does not seem to be a comprehensive UML profile specifically targeted towards modeling safety-related concepts driven from safety standards (see section 4). If it existed, such a UML profile would help bridge the gap between the system's safety assessment (performed by safety engineers) and the software design (performed by software engineers). The safety engineers need not necessarily understand the UML profile, but they will need the information that is reprented by the profile. See Figure 4 for an illustration.

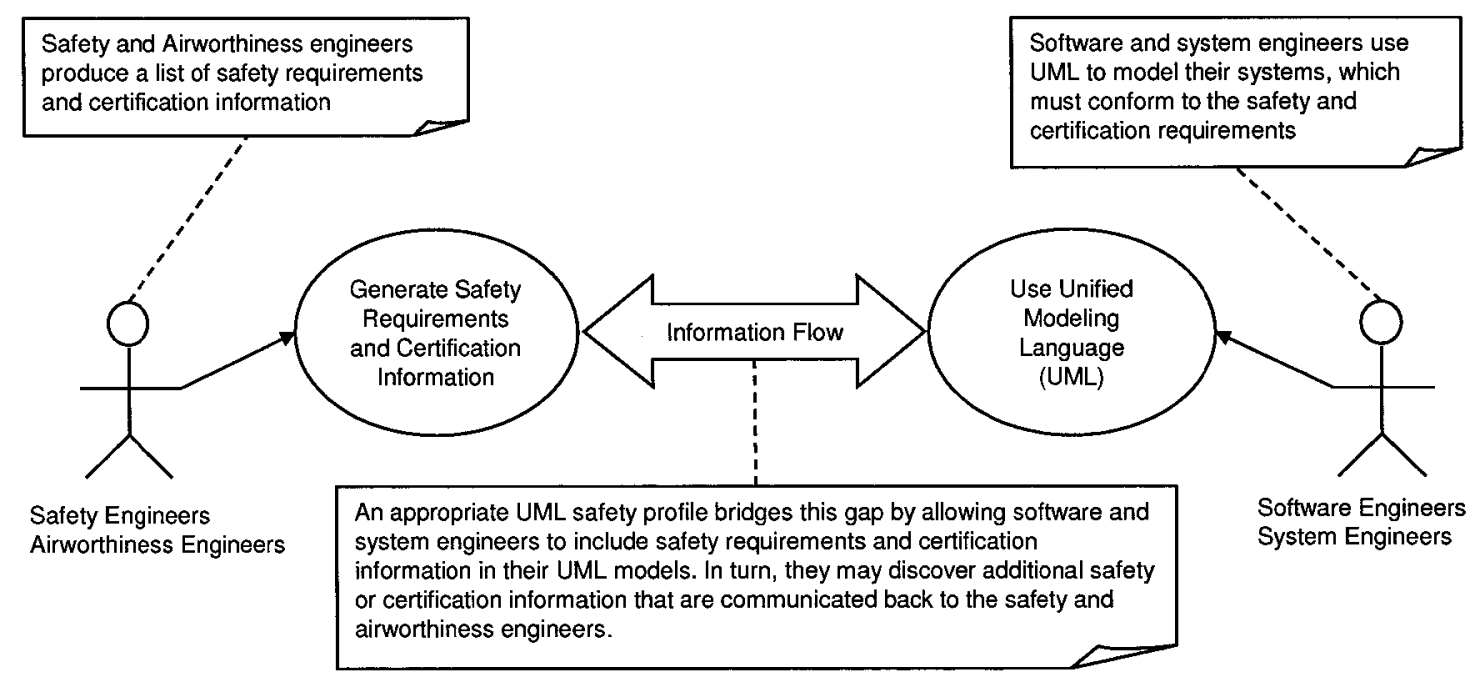

Figure 4: Role of a UML safety profile in the development process.

\subsubsection{Disadvantages}

Using this approach has the following disadvantages: 
1. Is an extra step to the development process: Most software development processes do not require documenting safety-related properties on design models. Therefore, this approach will be an additional step to most software development processes.

2. Requires that software engineers consider a topic they are likely to have little experience in - safety: Safety is a specialized topic of software engineering. Since software engineers may come from backgrounds where they were developing non-safety-critical systems, they may not have sufficient knowledge and experience in this topic.

\subsubsection{Advantages}

Using this approach has the following advantages:

1. Results in safer and higher quality software: By representing safety requirements and constraints on UML diagrams, the engineers are forced to consider them and use them to design and implement the systems.

2. Enables the possibility of reusing the results of a safety assessment through software models: Performing a safety assessment, like a risk assessment, is costly and time consuming. Therefore, representing the results of a safety assessment on UML diagrams allows it to be reused whenever the design is reused instead of reassessing the new or modified system from scratch. This is important when an organization is designing similar systems. 
3. Improves communication between safety engineers and software engineers: Software developers will have a better understanding of the safety engineers' requirements. This will allow software engineers to better understand and review the results of the safety assessment. As a result, software engineers will be able to provide feedback regarding the safety assessment.

4. Improves documentation of safety-related properties: Appropriate documentation is required by many standards including the MIL-STD-498 [11], and its replacement IEEE/EIA 12207 [12], which is one of the most referenced software development documentation standards. Documenting the results of a safety assessment in UML provides a step in that direction.

5. Increases participation of software engineers in the safety assessment: This approach drives software engineers to think about the results of the safety assessment because it is represented in UML. Therefore, they will implicitly perform additional analyses as part of their job to design and implement the software. As a result, they may discover additional safety requirements and constraints, which can then be communicated back to the safety assessment process.

6. Increases level of formalism: The results of the safety assessment will be represented using methods that are more formal than plain text English. This helps introduce the benefits of using formal methods, such as being able to detect conflicting or ambiguous requirements. In addition, this will make it easier for 
tools to use this information to help the engineers design, implement, and verify systems.

7. Maps safety-related properties to source code: This approach allows safetyrelated properties to be represented in UML models. In the software development process, the source code is directly traceable to UML models. Therefore, this approach allows safety-related properties to be mapped to the source code through the UML designs. This improves traceability, which is often required by many standards and is critical in large programmes.

8. Lower cost to use since UML is known in the software community: A UML safety profile builds on the engineers' knowledge of UML. Therefore, there is no additional cost to train the software engineers to use UML. They only need to be aware of the UML profile specifics and how it builds on top of UML. This should be inexpensive as software engineers should already be experienced with UML and the tools that support it.

9. Emphasizes a "develop in safety" culture: In her book on software safety [1], Nancy Leveson stressed the need for a "develop in safety" culture in organizations. This UML approach emphasizes Leveson's point as it involves the software engineers and developers in the safety aspects of the software.

10. Supports the MDA initiative: The MDA initiative [3] is a promising approach that, in conjunction with UML, seems to be the future of software development. It argues that design should be modeled appropriately first, which then allows the engineers to forward-generate the implementation (e.g. software code) from the 
high-level designs using appropriate computer tools. This is a very active research area that is projected to be the future of the development process as it will increase the level of automation. Such a UML profile allows describing the safety aspects in the software model, which would in turn be used in the MDA approach.

11. Makes life of engineers easier: For the engineers, this means that relevant safety information will be represented in a language that they understand, namely UML, rather than having to read other informal documents that risk being ambiguous.

\subsubsection{Requirements of an Effective UML Profile}

Based on the previous discussion, we identify that the requirements of an effective UML profile are:

REQ 1 The profile shall provide insight into the software's compliance aspects with airworthiness, and such evidence of compliance shall be obtainable at low costs: In essence, the cumulative cost of training the engineers to use the profile and extracting regular software compliance reports for progress tracking shall be less than the cost of collecting software compliance evidence when the profile is not used.

REQ 2 The profile shall allow the software engineers to relate technical solutions to the specific airworthiness requirements: Therefore, it shall be possible to exactly determine the software design and source code units that are responsible for satisfying each airworthiness requirement. 
REQ 3 The profile shall have clear language semantics with respect to safety and airworthiness: For example, the profile shall not assume that safety is simply reliability or some other concept - it shall recognize safety and airworthiness as a separate quality domain. This will enable better representation of requirements as well as improve the software engineers' understanding and ability to distinguish between safety and other concepts such as reliability.

REQ4 The profile shall model technical solutions using machine-readable extension mechanisms that increase the level of formalism: In other words, specific domain stereotypes, tags, and constraints shall be preferable to general purpose comments. In addition, the extensions shall allow software engineers to identify various kinds of technical solutions. Examples of technical solutions include safety monitors and multiple-version dissimilar software. This is particularly useful when developing software under high software airworthiness (see section 2.2) levels when formal methods are more likely to be used, and when models are reused across projects.

REQ 5 The profile shall support, or be easily scalable to, developing software under high airworthiness levels: Different airworthiness levels have different compliance requirements. For example, level $\mathrm{C}$ and above require tracing source code to low-level requirements and tracing low-level requirements to high-level requirements, whereas levels $\mathrm{D}$ and $\mathrm{E}$ do not have such requirements [4]. As another example, high software levels require checking that requirements are compatible with the target computers 
on which the software is deployed. Refer to annex A in [4] for a list of objectives per software level.

REQ 6 The profile shall favour language semantics that are meaningful to both software engineers and airworthiness engineers for concepts that both engineering groups need to discuss: Therefore, it will improve communication between the two groups.

REQ7 The profile shall favour representing airworthiness requirements using machine-readable extension mechanisms: Thus, it shall define some airworthiness-specific stereotypes, tags, and constraints that are parameterizable. Again, this is particularly useful when developing software under high-software levels because that is when formal methods are more likely to be used due to their ability to prove correctness. In addition, this is also useful when models are to be reused across projects. 


\section{SAFETY-RELATED CONCEPTS}

This section describes airworthiness-related concepts that were extracted from the airworthiness standard, RTCA DO-178B [4]. Since airworthiness is a subset of safety (see end of section 2.2), all of the identified airworthiness-related concepts are safetyrelated concepts. This does not necessarily mean that those concepts are the only ones needed for all safety-critical applications including transportation, medical, nuclear, and other industries. However, they should be enough for any development under the airworthiness standard [4], which is the goal of this research.

Since airworthiness is a subset of safety, airworthiness-related concepts will be referred to with their general term, safety-related concepts, in the remainder of this document. This is to emphasize that they are not restricted to airworthiness even though they resulted from analysing the airworthiness standard.

Ensuring software safety has many concerns that impact other qualities of service. As a result, the extracted safety-related concepts form a long list of concepts related to many concerns and qualities of service. Hence, it is important to group concepts that are most related together, which will also improve clarity and give the reader the general goal of each concept. For example, consider the following safety-related requirement:

An aircraft shall ensure that its landing wheels are deployed when the aircraft's altitude is less than 100 meters 
The following concerns are relevant for designing and implementing the safety-critical software assigned with the above requirement:

1. It must be safe so that the aircraft does not hit the ground with the landing wheels not deployed (i.e. when they are in their compartments). Software safety is the ability of the software to execute within its system context without contributing to hazards, which may lead to accidents or losses [1].

2. It must be reliable so that it correctly implements the requirement by ensuring that landing wheels are deployed when the altitude is less than 100 meters. Software reliability is the capability of the software system to offer continued service, or more specifically to maintain a specified level of performance when used under specified conditions [5].

3. It must have high integrity by providing precise and accurate results so that an altitude of less than 100 meters is not interpreted to be larger than 100 meters (and thus causing the aircraft not to deploy its wheels when it should). Software integrity is the capability of the software system to produce the expected quality of service of the correct functionality delivered by the software [5].

4. It must have high performance capabilities, so that the landing wheels are deployed when the aircraft's altitude drops to less than 100 meters even when it does so quickly in a fast descent such as the case in an emergency. Software performance refers to the timeliness aspects of how software systems behave, and sometimes it refers to the relationship between the services provided and the utilization of resources [5]. 
5. It must provide concurrent control so that the landing wheels are deployed even if pilot is already using the system to perform other functionality through the user interface. Software concurrency refers to the concurrent and temporal consistency of data and software elements [5].

6. It must be certifiable as vendors of such safety-critical systems often require them to be certified by an external third-party certification authority. Certification is the legal recognition by the certification authority that a product, service, organization or person complies with the requirements [4]

7. It must be properly designed so that non-critical software, such as a language dictionary, is decoupled from highly safety-critical software, such as the software interfacing with the landing wheels and the altitude sensors, to ensure that software bugs in non-critical software do not cause the critical software to fail.

8. It must be independent of the various software configurations that may be loaded into the system to configure it such as English and French dictionaries to customize the user interface. Software configuration, which is not the same as software configuration management, is the concept of having multiple software configurations or settings, each of which has a different set of functionalities and behaviours than the others.

Notice that while the concerns above describe various software quality categories, they all contribute to safety in some way. With that in mind, it is important to classify all of the extracted safety-related concepts into quality categories that best describe each related group of concepts. Those categories help the reader have a better understanding of the 
general goal of each concept, and they provide guidance on what to look for when attempting to describe a specific concept.

\subsection{Concept Identification and Categorization}

The selected standard, the airworthiness RTCA DO-178B standard [4], was analysed and a list of safety-related concepts was extracted. Those safety-related concepts are not solely safety concepts, and hence the rationale behind using the term "safety-related concepts" rather than simply "safety concepts" - in fact, many of those concepts are primarily non-safety concepts, such as reliability concepts, fault-tolerance concepts, certification concepts, and others. The "primarily" keyword identifies the category with which a concept is most associated. For example, fault tolerance is associated with reliability and, therefore, is a primarily reliability concept that also affects safety in some way.

To clarify and group related concepts together, the extracted safety-related concepts are classified in this research into the following safety-related quality categories:

1. Primarily safety concepts: The concepts listed in this category are software concepts that describe the software's safety aspects in the context of the system in which it is used. Software safety is the ability of the software to execute within its system context without contributing to hazards, which may lead to accidents or losses [1]. Examples of these concepts include safety-monitoring techniques and software levels. These concepts are listed and described in section C.1 of Appendix C. 
2. Primarily reliability concepts: The concepts listed in this category are software concepts that describe the software's reliability aspects. However, they also impact the safety of the software because of the relationship between software's reliability and safety - reliability measures the probability of failure, whereas safety measures the consequences of those failures [5]. Software reliability is the capability of the software system to offer continued service, or more specifically to maintain a specified level of performance when used under specified conditions [5]. Examples of primarily reliability concepts include exception handling and fault tolerance. These concepts are listed and described in section C.2 of Appendix C.

3. Primarily integrity concepts: The concepts listed in this category are software concepts that describe the software's integrity aspects. However, they also impact the safety of the software because of the relationship between software's integrity and safety. Software integrity is the capability of the software system to produce the expected quality of service of the correct functionality delivered by the software [5]. Examples of primarily integrity concepts include accuracy and precision: Inaccurate data may cause the safety-critical system to behave in a nonsafe way. As an example, consider an aircraft the needs to know when to deploy the landing wheels - it must have an accurate value of the altitude so that it deploys the landing wheels when it should. These concepts are listed and described in section C.3 of Appendix C.

4. Primarily performance concepts: The concepts listed in this category are software concepts that describe the software's performance aspects. However, they also 
impact the safety of the software because of the relationship between software's performance and safety. Software performance refers to the timeliness aspects of how software systems behave, and sometimes it refers to the relationship between the services provided and the utilization of resources [5]. In this document, performance concepts include schedulability and time concepts. Examples of primarily performance concepts include scheduling strategies (e.g. round robin, rate monotonic) and time-related (e.g. filters) functions. For example, an aircraft's scheduling software must schedule safety-critical tasks with priorities higher than non safety-critical tasks. These concepts are listed and described in section C.4 of Appendix C.

5. Primarily concurrency concepts: The concepts listed in this category are software concepts that describe the software's concurrency aspects. However, they also impact the safety of the software because of the relationship between software's concurrency and safety. Software concurrency refers to the concurrent and temporal consistency of data and software elements [5]. Examples of primarily concurrency concepts include multi-tasking and active software components, which may be safety-critical. These concepts are listed and described in section C.5 of Appendix C.

6. Primarily certification concepts: The concepts listed in this category are software concepts that describe the software's certification aspects. However, they also impact the safety of the software because of the relationship between software's certification and safety. Certification is the legal recognition by the certification authority that a product, service, organization or person complies with some 
requirements. Such certification comprises the activity of technically checking the product, service, organization or person and the formal recognition of compliance with the applicable requirements by issue of a certificate, license, approval or other documents as required by national laws and procedures [4]. Examples of primarily certification concepts include certification requirements (e.g. specifying hardware/software interfaces) and software traceability. These concepts are listed and described in section C.6 of Appendix C.

7. Primarily design concepts: The concepts listed in this category are software concepts that describe the software's design aspects. However, they also impact the safety of the software because of the relationship between software's design and safety. These generally impact areas such as the quality, clarity, and maintainability of the model and the source code. The impact of these decisions on the use of the end product is usually less defined than those of other quality categories. Examples of primarily design concepts include software coupling and software complexity. These concepts are listed and described in section C.7 of Appendix C.

8. Primarily configuration concepts: The concepts listed in this category are software concepts that describe the software's configuration aspects. However, they also impact the safety of the software because developing multipleconfiguration software is more challenging than developing single configuration software due to the changing behaviour. As a result, it is more challenging to fully predict the behaviour of the software, especially when the user of the software can change the configuration (e.g. by changing memory bits or loading customized 
look up tables). Software configuration, which is not the same as software configuration management, is the concept of having multiple software configurations or settings, each of which has a different set of functionalities and behaviours than the others. Choosing the desired software configuration is usually performed by the user rather than the software developer. Examples of primarily configuration concepts include option-selectable software and user-modifiable software. These concepts are listed and described in section C.8 of Appendix C.

An 8-dimensional space is needed to fully describe the relationships across all safetyrelated quality categories identified above. For simplicity, let us assume that reliability, integrity, performance, concurrency, certification, design, and configuration concepts sets form mutually exclusive sets. If we fix values for 6 dimensions, then we can arrive at a 2dimensional view that shows the relationship between safety and each of the other identified safety-related quality categories independently of their cross relationships. This view is somehow simplistic but it is useful for illustration purposes. It is shown in Figure 5 below.

Figure 5 shows that the identified quality categories are related to safety. In other words, there exist concepts that are present in more than one quality category, one of which is the safety quality category. All of the concepts extracted from the airworthiness standard belong to at least the safety quality category. In addition, some of those concepts also belong to categories other than the safety category. For example, a "shared resource" is a "concurrency" concept because multiple flows of control may be competing for access to it. This will also impact the performance aspects of the software. Moreover, it is also relevant to the "design" aspects of the software because the way it will be used is a result 
of design decisions. Finally, all of these aspects might impact the "safety"-aspects if one or more of the competing flows of control are executing a safety-critical software section.

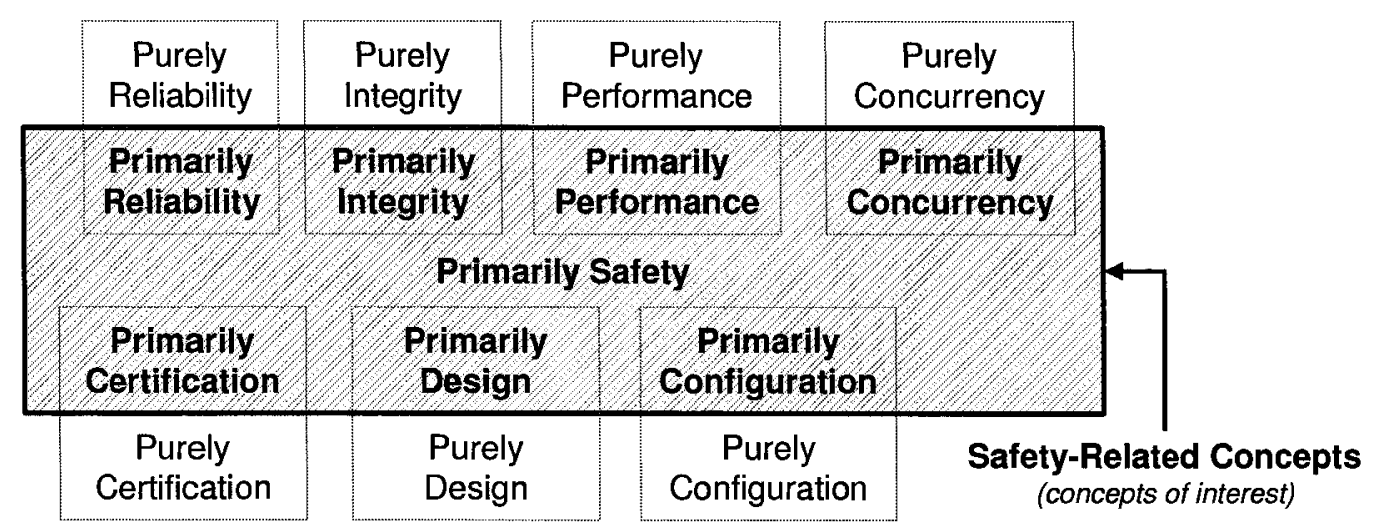

Figure 5: Relationship between safety and other safety-related quality categories assuming that those categories form mutually exclusive sets.

Regardless of its category, each concept can be one of three different kinds:

1. It can be a "safety entity", which is software that may contribute, positively or negatively, to the overall safety level of the system or software. Furthermore, a safety entity can interact with another safety entity. An example of a safety entity would be identifying a particular software component as a fault monitor. A fault monitor (one safety entity) is used to monitor particular functions (other safety entities) to detect faults that could occur and cause the system to enter a hazardous state. If a fault monitor detects such a fault, it could then perform appropriate actions to prevent the system from entering hazardous states. Therefore, the fault monitor is a safety entity whose purpose is to increase the overall level of safety of the system. 
2. It can be a "safety attribute", which is a concept that describes a safety property, such as the safety level or impact on safety, of safety entities. For example, the fault monitor described above may be used to detect specific faults or unusual results and behaviours. For example, what if a particular software function that is used to indicate the altitude of the aircraft provides output that says that the aircraft has a negative altitude? This is clearly an incorrect result. Assume that a fault resulting in this scenario is detectable by the fault monitor described earlier. In this case, this specific fault can be used as a safety attribute of the fault monitor. In other words, the complete specification of the fault monitor safety entity will explicitly state through one of its safety attributes that it can detect this fault.

3. It can be a "safety method", which is an activity, technique, or a process that may measure or impact, positively or negatively, the safety level of safety entities. For example, a scenario that describes the fault monitoring example above covers many concepts such as fault monitoring, faults, and possibly many others that are related to the ability of monitoring software against faults that they may cause. Therefore, all that discussion is centred around one concept, namely "fault monitoring". Thus, that scenario describes one "safety method" that is fault monitoring.

The use of the word "safety" in safety entity, safety attribute, and safety method only means that those concepts are safety-related. It does not assume that they increase the level of safety. 
Figure 6 (a) formalises those definitions through a conceptual conceptual model. Notice that each safety entity is described through safety attributes. Safety entities may interact with each other, and they may implement safety methods (usually those that positively impact safety). Safety methods may measure or impact, positively or negatively, the safety level of safety entities. Figure 6 (b) describes the fault monitoring example presented above, which is an instance of the conceptual model in Figure 6 (a).

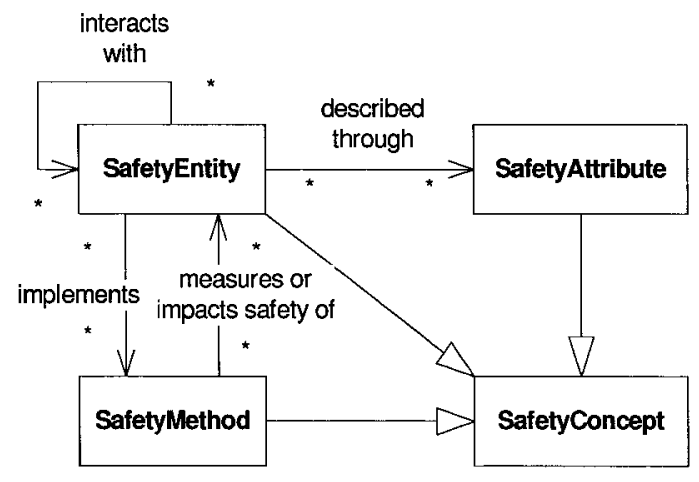

(a) Model

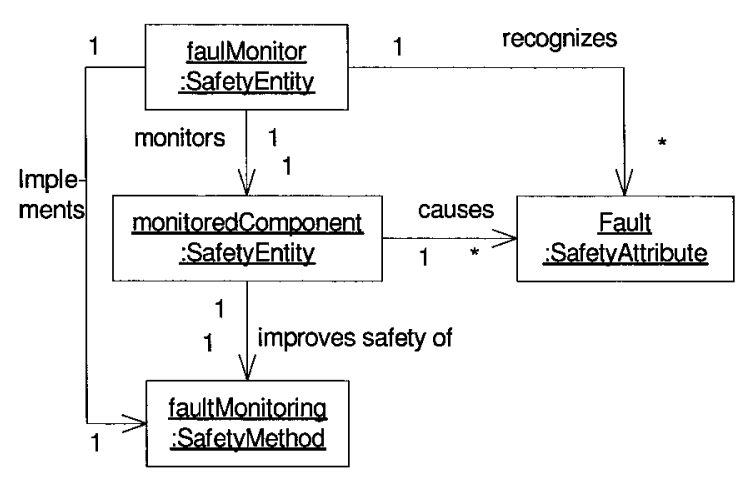

(b) Fault monitoring example

Figure 6: Relationship across safety entities, attributes, and methods.

The safety-related concepts for each category are listed in Appendix C. They are described according to the context in which they appear within the airworthiness standard, RTCA DO-178B [4]. Those concepts are then refined into a terminology that is more suitable for modeling software. The refined concepts, along with their descriptions and their inter-concept relationships, are presented in section 3.2.

\subsection{Concept Refinement}

Section 3.1 and Appendix C introduced 65 safety-related concepts that were found relevant for developing airworthiness-compliant software. They were then grouped 
according to their software quality category. In this section, those concepts are refined for the following reasons:

1. Removing duplicate concepts: In some instances, seemingly different concepts appeared in the airworthiness standard where, in reality, they can be represented using a single concept or term. This is because the same fundamental software concept can appear in different forms in airworthiness-related applications. Examples are presented below.

Consider, for example, the two following primarily reliability concepts exactly as they appear in the airworthiness standard: Multiple-Version Dissimilar Software and Software Redundancy (see Appendix C.2 for definitions). They revolve around the same software concept, which is that of using multiple software components that have the same functionality but different implementations. It would be unnecessarily confusing to use two different terms to denote the same concept. As a result, a single refined term is provided to model both of those concepts, which is the "replicated" concept to denote software replication or redundancy.

2. Grouping concepts: Some concepts are in fact examples of a more general concept.

Consider the following concepts: Safety Monitoring, Loadable Software Indicator, Safeguard, Safety Feature (primarily safety concepts defined in Appendix C.1); Error Detection, Fault Detection, Fault Containment (primarily reliability concepts defined in Appendix C.2); Integrity Check, Software Protector (primary integrity concepts defined in Appendix C.3); Error Prevention 
(primarily design concept defined in Appendix C.7). They are applications of a single software-concept that is "Monitor". A "monitor" monitors the activity of other software components to detect unusual, potentially hazardous, events.

3. Precise definition of details: Presenting each concept is a single entry in a list may be misleading by giving the impression that it is the smallest level of detail. In reality, each concept has many attributes that describe it, with each attribute describing a single aspect of the concept. Examples are presented below.

For example, "Safety Requirement" is identified as a (primarily safety) safetyrelated concept (Appendix C.1). However, it does not mention the specification of safety requirements. When refining the "Safety Requirement" concept, we give it an attribute called "specification" that can be used to specify the details of the requirement. Therefore, a concept's attribute is used to describe a specific aspect or detail of it.

The rest of this section shows how the concepts and their relationships have been formalized under the form of a conceptual model, i.e., a UML class diagram (section 3.2.1), describes the template we use to specify the concepts (section 3.2.2), and then actually specifies the concepts (section 3.2.3). Appendix D provides additional details on the relationships between concepts.

\subsubsection{Conceptual Model}

Before describing the details of the concepts, we introduce a conceptual model to list the refined concepts and formalize the relationships among them (Figure 7-its elements are 
further described in section 3.2.3). Thus, the motivations for defining a conceptual model for the refined concepts are:

1. It introduces a high-level presentation of the concepts and their relationships, thus leaving out most attributes that are considered low-level details. This helps the reader better understand the concepts and their relationships.

2. It formalises the relationships across the concepts, for instance by specifying multiplicities on relationships.

3. It makes the definition and the use of the UML profile's extensions (stereotypes, tagged values, and constraints) in section 5 easier because the refined concepts are designed to be more appropriate from a modeling point of view. Thus, many of the profile's extensions refer back to the refined concepts. In fact, the profile's stereotypes and tagged values are based on the refined concepts, their attributes, and their relationships.

\subsubsection{Concept Details}

In this section, we describe the template we use in section 3.2.3 to define the concepts in Figure 7. Each of the following describes one characteristic of a concept:

1. Definition: This presents a definition for the concept. It describes the concept and gives its general purpose. 


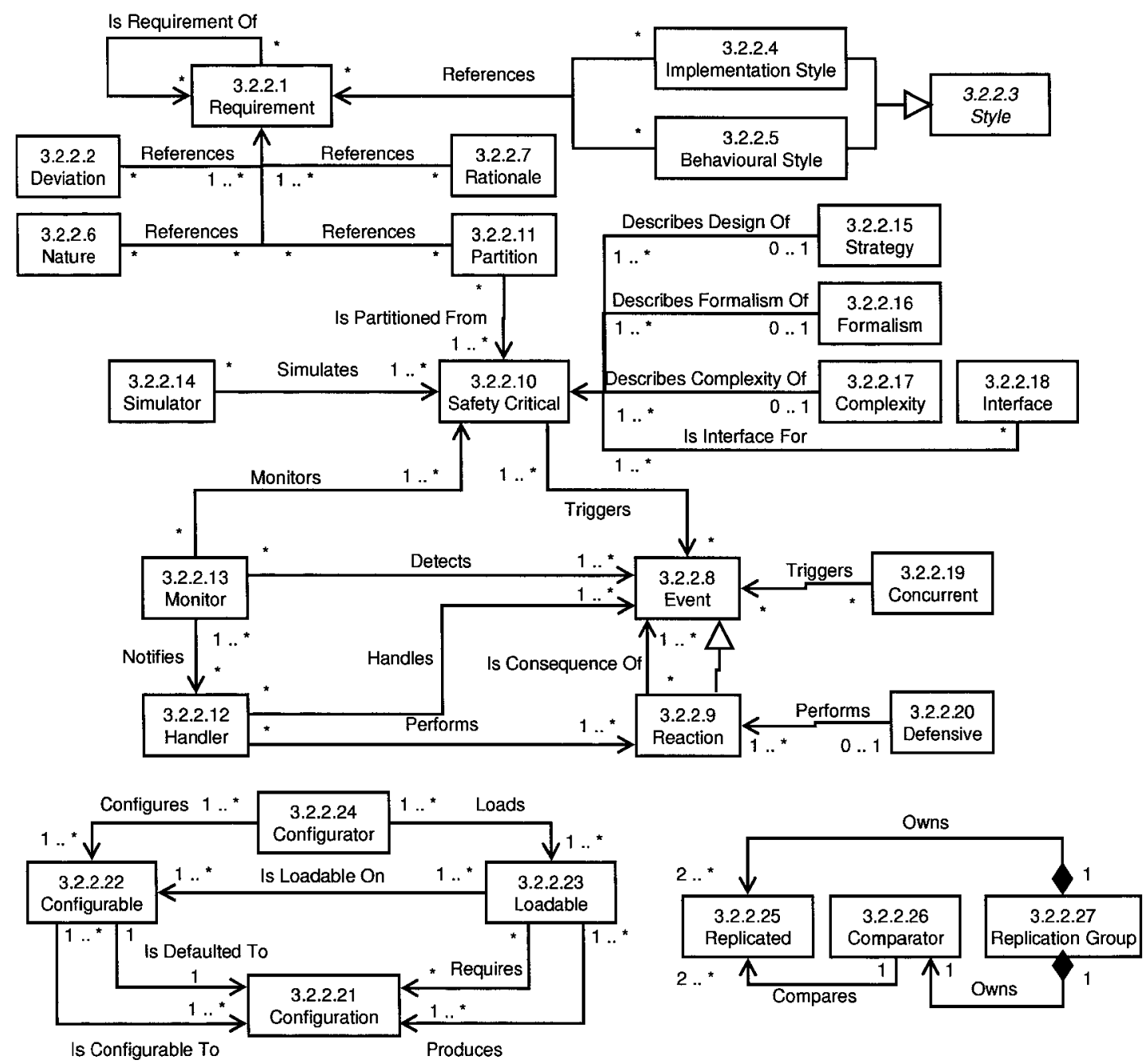

Figure 7: Meta-model for the refined safety-related concepts.

2. Attributes: This lists and describes the attributes for the concept. Each attribute describes a specific aspect of the concept. A name, description, and examples are provided for each concept.

3. Relationships: This lists and describes the relationships that the concept has with other concepts. A name and a description, which includes the end multiplicities, are provided for each concept's relationships. 
4. Original Safety-Related Concepts: This lists the original safety-related concepts, which were extracted from the airworthiness standard, RTCA DO-178B [4], and presented in section 3.1 (and Appendix C), that this concept represents. Thus, this information serves as additional justification for the concept, its attributes, and its relationships.

The attributes of each concept are presented in a table. For example, the attributes of the "Safety Critical" (section 3.2.3.10) refined concept are presented as follows:

\begin{tabular}{|l|l|l|}
\hline \multicolumn{1}{|c|}{ Name } & \multicolumn{1}{|c|}{ Description } & \multicolumn{1}{|c|}{ Examples } \\
\hline Criticality Level & $\begin{array}{l}\text { Indicates the level of criticality (e.g. } \\
\text { airworthiness level, Safety Integrity } \\
\text { Level (SIL)), on some pre-defined } \\
\text { scale, such as the software level or } \\
\text { the failure condition category }\end{array}$ & $\begin{array}{l}\text { For IEC "SIL 1", "SIL 2", "SIL } \\
\text { 3", "SIL 4" }\end{array}$ \\
& $\begin{array}{l}\text { Indicates the level of confidence, on } \\
\text { some pre-defined scale, that the } \\
\text { criticality level is satisfied }\end{array}$ & $\begin{array}{l}\text { "High", } \\
\text { Confidence Level }\end{array}$ \\
\hline
\end{tabular}

Each row describes a single concept attribute. The first column (Name) specifies the name of the attribute, the second column (Description) describes the attribute, and the third column (Examples) provides examples for the value of the attribute.

Similarly, the relationships of each concept are presented in a table. For example, the relationships of the "Safety Critical" (section 3.2.3.10) refined concept are presented as follows: 


\begin{tabular}{|l|l|}
\hline \multicolumn{1}{|c|}{ Name } & \multicolumn{1}{c|}{ Description } \\
\hline Triggers & $\begin{array}{l}\text { Identifies zero or more "Event" instance that the "Safety Critical" } \\
\text { instance triggers }\end{array}$ \\
\hline
\end{tabular}

Each row describes a single concept relationship. The first column (Name) specifies the name of the relationship, and the second column (Description) describes the relationship. The description of the relationship identifies the concept at the other end of the relationship as well as its multiplicity.

\subsubsection{Concepts Specifications}

The refined concepts are listed below in sections 3.2.3.1 to 3.2.3.27 in an order, different from the alphabetical order, that we think will help the reader better understand them and their relationships. For example, concept $\mathrm{A}$ is listed before concept $\mathrm{B}$ if concept $\mathrm{B}$ references or depends on concept $\mathrm{A}$.

Some of the concepts' attributes presented below are specific to the developed system or the development project. This is common for attributes whose type is an enumeration. In such cases, this research does not attempt to define all the possible values. However, it does present examples on what they could be. It is up to the software developers to define the enumeration values that are relevant to the system being developed.

The term "design element" is used to indicate "a portion of the design" such as a class, operation, collaboration (i.e. diagram), or relationship between classes. The design element can be either hardware or software. 


\subsubsection{Requirement}

Definiton:

The "Requirement" concept specifies a requirement that must be met. The requirement need not necessarily be a safety requirement - it can be any functional or non-functional requirements. It may be traceable to another requirement, which is often a higher level one. This enables the concept of requirements traceability, which is a key element in the software development process.

\section{Attributes:}

\begin{tabular}{|c|c|c|}
\hline Name & Description & Examples \\
\hline ID & A unique ID for this requirement & $\begin{array}{l}\text { "REQ 1", “REQ 2", } \\
\text { "FREQ 1", “SREQ 10", } \\
\text {... etc }\end{array}$ \\
\hline Kind & The kind of this requirement & $\begin{array}{l}\text { "Functional", "Safety", } \\
\text { "Reliability", "Integrity", } \\
\text { "Performance", } \\
\text { "Concurrency", } \\
\text { "Certification", "Design", } \\
\text { "Configuration", } \\
\text { "Derived", ... etc }\end{array}$ \\
\hline Specification & $\begin{array}{l}\text { The actual requirement's } \\
\text { specification }\end{array}$ & $\begin{array}{l}\text { "Radar Output is Poisson } \\
\text { with Lambda }=20 \mathrm{~ms} \text { ", } \\
\text { "Levels of Code Nesting } \\
<5 ", \ldots \text { etc }\end{array}$ \\
\hline
\end{tabular}

\section{Relationships:}

\begin{tabular}{|l|l|}
\hline \multicolumn{1}{|c|}{ Name } & \multicolumn{1}{|c|}{ Description } \\
\hline Is Requirement Of & $\begin{array}{l}\text { Identifies zero or more, usually higher-level, "Requirement" } \\
\text { instances to which this "Requirement" instance can be traced }\end{array}$ \\
\hline
\end{tabular}


Original Safety-Related Concepts:

Safety Requirement, Certification Requirement, Derived Requirement, Design by

\section{Contract}

\subsubsection{Deviation}

\section{Definiton:}

The "Deviation" concept identifies a design deviation from a plan, standard, or requirement (3.2.3.1). Deviations are important to note as they must be submitted to the certification authorities according to the airworthiness standard RTCA DO178B [4].

\section{Attributes:}

\begin{tabular}{|l|l|lr|}
\hline \multicolumn{1}{|c|}{ Name } & \multicolumn{1}{|c|}{ Description } & \multicolumn{1}{|c|}{ Examples } \\
\hline Kind & $\begin{array}{l}\text { The kind of this deviation. This } \\
\text { generally specifies the deviation } \\
\text { action or decision }\end{array}$ & $\begin{array}{l}\text { Rsing } \\
\text { Algorithm", "Using } \\
\text { Dynamic Memory", .. } \\
\text { etc }\end{array}$ \\
\hline Explanation & $\begin{array}{l}\text { Specifies how, or why, this is a "Kalman filter is } \\
\text { deviation from the reference } \\
\text { requirements (see relationships } \\
\text { below) }\end{array}$ & $\begin{array}{l}\text { recursive so using } \\
\text { the implementation", .. } \\
\text { etc }\end{array}$ \\
\hline
\end{tabular}

\section{Relationships:}

\begin{tabular}{|l|l|}
\hline \multicolumn{1}{|c|}{ Name } & \multicolumn{1}{c|}{ Description } \\
\hline References & $\begin{array}{l}\text { Identifies one or more "Requirement" (3.2.3.1) instances from } \\
\text { which the "Deviation" instance deviates }\end{array}$ \\
\hline
\end{tabular}

Original Safety-Related Concepts:

Deviation 


\subsubsection{Style}

\section{Definiton:}

The "Style" concept is an abstract concept indicating an implementation or a behavioural style. It does not capture any information, but it serves as a base class for other concepts.

Attributes:

None

\section{Relationships:}

None

Original Safety-Related Concepts:

Implementation Style, Time-Related, State-Related

\subsubsection{ImplementationStyle}

\section{Definiton:}

The "ImplementationStyle" concept identifies a style that is used to implement a design. A development standard should define which styles are permitted and which ones are not.

Attributes:

\begin{tabular}{|l|l|l|}
\hline \multicolumn{1}{|c|}{ Name } & \multicolumn{1}{|c|}{ Description } & \multicolumn{1}{|c|}{ Examples } \\
\hline Kind & The kind of this implementation style & "Recursive", "Unbounded \\
& & Loop", "Compacted \\
& & Expression", "Dynamic \\
& & Memory", "Data Alias", \\
& & \\
& & \\
& & \\
& & \\
& & \\
\end{tabular}




\begin{tabular}{|l|l|l|}
\hline \multicolumn{1}{|c|}{ Name } & \multicolumn{1}{|c|}{ Description } & \multicolumn{1}{|c|}{ Examples } \\
\hline Parameters & $\begin{array}{l}\text { Describes additional details of the "Dynamic memory } \\
\text { implementation style. It is generally } \\
\text { an expression whose meaning is } \\
\text { dependent on the Kind of the } \\
\text { implementation style }\end{array}$ & $\begin{array}{l}\text { allocation frequency }= \\
\text { Poisson with Lambda }= \\
15 \text { seconds",... etc }\end{array}$ \\
\hline Explanation & $\begin{array}{l}\text { Specifies how this implementation } \\
\text { style conforms to, or deviates from, } \\
\text { the reference requirements (see } \\
\text { relationships below) }\end{array}$ & $\begin{array}{l}\text { Using dynamic memory } \\
\text { because 90\% of the time } \\
\text { only 10\% of the } \\
\text { maximum memory space } \\
\text { will be needed (which } \\
\text { would be required if static } \\
\text { memory is used). This } \\
\text { improves performance", } \\
\ldots \text { etc static }\end{array}$ \\
\hline
\end{tabular}

Relationships:

\begin{tabular}{|l|l|}
\hline \multicolumn{1}{|c|}{ Name } & \multicolumn{1}{|c|}{ Description } \\
\hline Is Child Class Of & $\begin{array}{l}\text { States that all "ImplementationStyle" instances are "Style" } \\
(3.2 .3 .3) \text { instances }\end{array}$ \\
\hline References & $\begin{array}{l}\text { Identifies zero or more "Requirement" (3.2.3.1) instances } \\
\text { indicating that the "ImplementationStyle" instance conforms to or } \\
\text { deviates from }\end{array}$ \\
\hline
\end{tabular}

Original Safety-Related Concepts:

Recursion, Compacted Expression, Dynamic Memory, Data Alias 


\subsubsection{BehaviouralStyle}

\section{Definiton:}

The "BehaviouralStyle" concept identifies and describes a behavioural style of a design. A development standard should define which styles are permitted and which ones or not..

\section{Attributes:}

\begin{tabular}{|c|c|c|}
\hline Name & Description & Examples \\
\hline Kind & The kind of thie behavioural style & $\begin{array}{l}\text { "Time-Related", "State- } \\
\text { Related", ... etc }\end{array}$ \\
\hline Parameters & $\begin{array}{l}\text { Describes additional details of the } \\
\text { behavioural style. It is generally an } \\
\text { expression whose meaning is } \\
\text { dependent on the Kind of the } \\
\text { behavioural style }\end{array}$ & $\begin{array}{l}\text { "Number of state machine } \\
\text { states }=10 " \text { ", "Number of } \\
\text { state transitions = } 20 " \text {, } \\
\text { "Frequency of state } \\
\text { changes = Periodic every } \\
1 \text { minute", ... etc }\end{array}$ \\
\hline Explanation & $\begin{array}{l}\text { Specifies how this behavioural style } \\
\text { conforms to, or deviates from, the } \\
\text { reference requirements (see } \\
\text { relationships below) }\end{array}$ & $\begin{array}{l}\text { "Frequency of state } \\
\text { changes is less than the } \\
\text { maximum value permitted } \\
\text { by REQ } 23 \text { ", ... etc }\end{array}$ \\
\hline
\end{tabular}

\section{Relationships:}

\begin{tabular}{|l|l|}
\hline \multicolumn{1}{|c|}{ Name } & \multicolumn{1}{|c|}{ Description } \\
\hline Is Child Class Of & $\begin{array}{l}\text { States that all "BehaviouralStyle" instances are "Style" (3.2.3.3) } \\
\text { instances }\end{array}$ \\
\hline References & $\begin{array}{l}\text { Identifies zero or more "Requirement" (3.2.3.1) instances } \\
\text { describing that the "BehaviouralStyle" instance conforms to or } \\
\text { deviates from }\end{array}$ \\
\hline
\end{tabular}


Original Safety-Related Concepts:

Time-Related, State-Related

\subsubsection{Nature}

\section{Definiton:}

The "Nature" concept describes the source for the design such as whether the actual software is purchased to meet the requirements, whether it was previously developed as part of another project or software system, or whether it is deactivated and does not get executed.

\section{Attributes:}

\begin{tabular}{|c|c|c|}
\hline Name & Description & Examples \\
\hline Kind & $\begin{array}{l}\text { The kind of the software's nature. It } \\
\text { is the primary attribute that describes } \\
\text { the actual software represented by } \\
\text { this concept }\end{array}$ & $\begin{array}{l}\text { "COTS", "Deactivated", } \\
\text { "Previously Developed", } \\
\text {... etc }\end{array}$ \\
\hline Explanation & $\begin{array}{l}\text { Specifies how the referenced } \\
\text { requirements are met by the nature of } \\
\text { this design (see relationships below) }\end{array}$ & $\begin{array}{l}\text { "This is a COTS software } \\
\text { component purchased } \\
\text { according to document } \\
\text { number } 1234567 \text { to meet } \\
\text { requirements REQ } 1- \\
\text { REQ } 10 ", \ldots \text { etc }\end{array}$ \\
\hline
\end{tabular}

\section{Relationships:}

\begin{tabular}{|l|l|}
\hline \multicolumn{1}{|c|}{ Name } & \multicolumn{1}{c|}{ Description } \\
\hline References & $\begin{array}{l}\text { Identifies zero or more "Requirement" (3.2.3.1) instances that are } \\
\text { the reasons for the "Nature" instance's existence }\end{array}$ \\
\hline
\end{tabular}

\section{Original Safety-Related Concepts:}

COTS Software, Deactivated Code, Previously Developed Software 


\subsubsection{Rationale}

\section{Definiton:}

The "Rationale" concept specifies that a specific design exists to support another design element, or to fulfill specific requirements. It explicitly allows modelers to trace the design to specific requirements (3.2.3.1).

\section{Attributes:}

\begin{tabular}{|l|l|l|}
\hline \multicolumn{1}{|c|}{ Name } & \multicolumn{1}{|c|}{ Description } & \multicolumn{1}{|c|}{ Examples } \\
\hline Explanation & $\begin{array}{l}\text { Specifies how the design decision is a "This class lists safe flight } \\
\text { solution for the referenced } \\
\text { requirements }\end{array}$ & $\begin{array}{l}\text { paths for an aircraft, } \\
\text { which is used to satisfy } \\
\text { safety requirements } \\
\end{array}$ \\
& SREQ 1, SREQ 2, and \\
& SREQ 3", .. etc \\
\hline
\end{tabular}

Relationships:

\begin{tabular}{|l|l|}
\hline \multicolumn{1}{|c|}{ Name } & \multicolumn{1}{c|}{ Description } \\
\hline References & $\begin{array}{l}\text { Identifies one or more "Requirement" (3.2.3.1) instances that are } \\
\text { the reasons for the "Rationale" instance's existence }\end{array}$ \\
\hline
\end{tabular}

\section{Original Safety-Related Concepts:}

Traceability

\subsubsection{Event}

\section{Definiton:}

The "Event" concept describes an event or action that may occur. An event may impact safety by either causing or removing hazards. It may also be caused internally by the system or it may be an external event. It does not need another event to trigger it. 
Attributes:

\begin{tabular}{|l|l|l|}
\hline \multicolumn{1}{|c|}{ Name } & \multicolumn{1}{|c|}{ Description } & \multicolumn{1}{|c|}{ Examples } \\
\hline Kind & The kind of this event & "External", "Internal",... \\
etc
\end{tabular}

\section{Relationships:}

None

\section{Original Safety-Related Concepts:}

Unsafe Action, Failure, Failure Condition, Fault, Error, Integrity Check 


\subsubsection{Reaction}

\section{Definiton:}

The "Reaction" concept describes a reaction to one or more events (3.2.3.8) that may occur. A reaction may impact safety by either causing or removing hazards. It is an event (3.2.3.8) in itself, but it always occurs in response to other events (3.2.3.8). It is a subclass of the event (3.2.3.8) concept to allow the possibility of chain reactions (i.e. there could be a reaction for a reaction).

\section{Attributes:}

\begin{tabular}{|c|c|c|}
\hline Name & Description & Examples \\
\hline Kind & Inherited from "Event" (3.2.3.8) & See "Event" (3.2.3.8) \\
\hline When & $\begin{array}{l}\text { Inherited from "Event" }(3.2 .3 .8) \text {. In } \\
\text { effect, this attribute -filters out } \\
\text { situations when the reaction will not } \\
\text { be performed as a result of the event } \\
\text { (3.2.3.8) occurrence. This may be } \\
\text { specified in a formal language }\end{array}$ & See "Event" (3.2.3.8) \\
\hline $\begin{array}{l}\text { Effect On Safety } \\
\text { Direction }\end{array}$ & Inherited from "Event" (3.2.3.8) & See "Event" (3.2.3.8) \\
\hline $\begin{array}{l}\text { Effect On Safety } \\
\text { Value }\end{array}$ & Inherited from "Event" (3.2.3.8) & See "Event" (3.2.3.8) \\
\hline $\begin{array}{l}\text { Effect on Safety } \\
\text { Context }\end{array}$ & Inherited from "Event" (3.2.3.8) & See "Event" (3.2.3.8) \\
\hline
\end{tabular}

Relationships:

\begin{tabular}{|l|l|}
\hline \multicolumn{1}{|c|}{ Name } & \multicolumn{1}{c|}{ Description } \\
\hline Is Child Class Of & States that all "Reaction" instances are also "Event" instances \\
\hline Is Consequence Of & $\begin{array}{l}\text { Identifies one or more "Event" (3.2.3.8) instances to which the } \\
\text { "Reaction" instance is a consequence of }\end{array}$ \\
\hline
\end{tabular}


Original Safety-Related Concepts:

Safety Response

\subsubsection{SafetyCritical}

Definiton:

The "SafetyCritical" concept represents a safety-critical design or element that impacts safety. It also identifies the safety or airworthiness level of design elements.

Attributes:

\begin{tabular}{|l|l|l|}
\hline \multicolumn{1}{|c|}{ Name } & \multicolumn{1}{|c|}{ Description } & \multicolumn{1}{|c|}{ Examples } \\
\hline Criticality Level & $\begin{array}{l}\text { Indicates the level of criticality (e.g. } \\
\text { airworthiness level, Safety Integrity } \\
\text { Level (SIL)), on some pre-defined } \\
\text { scale, such as the software level or } \\
\text { the failure condition category }\end{array}$ & $\begin{array}{l}\text { For } \text { "SIL 1", "SIL 2", "SIL } \\
\text { 3", "SIL 4" }\end{array}$ \\
\hline Confidence Level & $\begin{array}{l}\text { Indicates the level of confidence, on } \\
\text { some pre-defined scale, that the } \\
\text { criticality level is satisfied }\end{array}$ & $\begin{array}{l}\text { "High", } \\
\text { "Low", "80\%", "50\%", }\end{array}$ \\
\hline
\end{tabular}

Relationships:

\begin{tabular}{|l|l|}
\hline \multicolumn{1}{|c|}{ Name } & \multicolumn{1}{|c|}{ Description } \\
\hline Triggers & $\begin{array}{l}\text { Identifies zero or more "Event" (3.2.3.8) instance that the } \\
\text { "SafetyCritical" instance may trigger }\end{array}$ \\
\hline
\end{tabular}

Original Safety-Related Concepts:

Safety-Critical, Software Level, Level of Confidence, Failure Condition Category 


\subsubsection{Partition}

\section{Definiton:}

The "Partition" concept identifies a design partition that resulted from separating some design element from other design elements. Partitioning is a technique for providing isolation between functionally independent entities to contain and/or isolate faults and potentially reduce the effort of the verification process. It prevents specific interactions and cross-coupling interference [1]. Its key advantages are in separating safety-critical design elements that have different safety levels, so that the failure of the less critical entity does not result in the failure of the more critical entities.

\section{Attributes:}

\begin{tabular}{|c|c|c|}
\hline Name & Description & Examples \\
\hline Explanation & $\begin{array}{l}\text { Provides further details on the } \\
\text { reasons for the partitioning }\end{array}$ & $\begin{array}{l}\text { "Partitioned away from a } \\
\text { software component with } \\
\text { a higher airworthiness } \\
\text { level", ... etc }\end{array}$ \\
\hline
\end{tabular}

\section{Relationships:}

\begin{tabular}{|l|l|}
\hline \multicolumn{1}{|c|}{ Name } & \multicolumn{1}{|c|}{ Description } \\
\hline References & $\begin{array}{l}\text { Identifies zero or more "Requirement" (3.2.3.1) instances that } \\
\text { specify the reasons for the "Partition" instance's existence }\end{array}$ \\
\hline Is Partitioned From & $\begin{array}{l}\text { Identifies one or more "Safety Critical" (3.2.3.10) instances from } \\
\text { which this "Partition" instance was partitioned }\end{array}$ \\
\hline
\end{tabular}

Original Safety-Related Concepts:

Partitioning 


\subsubsection{Handler}

\section{Definiton:}

The "Handler" concept identifies a design element that handles events (3.2.3.8) that are detected by a monitor (3.2.3.13). A handler handles the events (3.2.3.8) by performing specific reactions (3.2.3.9) in response to the events (3.2.3.8).

Attributes:

None

Relationships:

\begin{tabular}{|l|l|}
\hline \multicolumn{1}{|c|}{ Name } & \multicolumn{1}{|c|}{ Description } \\
\hline Handles & $\begin{array}{l}\text { Identifies one or more "Event" (3.2.3.8) instances that the } \\
\text { "Handler" instance can handle by performing certain reactions }\end{array}$ \\
\hline Performs & $\begin{array}{l}\text { Identifies one or more "Reaction" (3.2.3.9) instances that the } \\
\text { "Handler" instance performs to handle events }\end{array}$ \\
\hline
\end{tabular}

Original Safety-Related Concepts:

Exception Handling, Fault Containment, Immunity, Software Protector, Safety Feature

\subsubsection{Monitor}

Definiton:

The "Monitor" concept identifies a design element that monitors other safetycritical (3.2.3.10) design elements for events (3.2.3.8). Detected events (3.2.3.8) are passed to handlers (3.2.3.12) for processing, which in turn invoke the appropriate reactions (3.2.3.9). 
Attributes:

\begin{tabular}{|l|l|l|}
\hline \multicolumn{1}{|c|}{ Name } & \multicolumn{1}{|c|}{ Description } & \multicolumn{1}{c|}{ Examples } \\
\hline Kind & The kind of this monitor, indicating & "Safety", "Reliability", \\
the quality of service that it monitors & "Integrity", \\
& & "Performance", \\
& & "Concurrency", \\
& & "Configuration",... etc \\
\hline
\end{tabular}

\section{Relationships:}

\begin{tabular}{|l|l|}
\hline \multicolumn{1}{|c|}{ Name } & \multicolumn{1}{c|}{ Description } \\
\hline Monitors & $\begin{array}{l}\text { Identifies one or more "Safety Critical" (3.2.3.10) instances that } \\
\text { the "Monitor" instance monitors for events }\end{array}$ \\
\hline Detects & $\begin{array}{l}\text { Identifies one or more "Event" (3.2.3.8) instances that the } \\
\text { "Monitor" instance detects }\end{array}$ \\
\hline Notifies & $\begin{array}{l}\text { Identifies zero or more "Handler" (3.2.3.12) instances that the } \\
\text { "Monitor" instance notifies when it detects events }\end{array}$ \\
\hline
\end{tabular}

Original Safety-Related Concepts:

Safety Monitoring, Error Detection, Fault Detection, Fault Containment, Error

Prevention, Integrity Check, Software Protector, Loadable Software Indicator, Safeguard, Safety Feature

\subsubsection{Simulator}

\section{Definiton:}

The "Simulator" concept identifies a design element that mimics the behaviour, usually in test mode, of another design element that will be used in the real system. For example, software simulators are common for hardware elements or other subsystems (hardware or software). Simulators are often used in developing large 
systems, they make the testing experience easier and more cost effective, and they play a key role in system integration labs [1].

Attributes:

\begin{tabular}{|l|l|l|}
\hline \multicolumn{1}{|c|}{ Name } & \multicolumn{1}{|c|}{ Description } & \multicolumn{1}{|c|}{ Examples } \\
\hline Parameters & $\begin{array}{l}\text { Specifies which behaviours are } \\
\text { simulated and how }\end{array}$ & $\begin{array}{l}\text { For a communication } \\
\text { subsystem simulator (e.g. } \\
\text { Radio Frequency (RF)): } \\
\text { "Messages received as } \\
\text { Poisson with Lambda } \\
\text { 100ms", "Message loss } \\
\text { frequency is Poisson with } \\
\text { Lambda }=250 \text { messages", } \\
\text {... etc }\end{array}$ \\
\hline
\end{tabular}

Relationships:

\begin{tabular}{|l|l|}
\hline \multicolumn{1}{|c|}{ Name } & \multicolumn{1}{c|}{ Description } \\
\hline Simulates & $\begin{array}{l}\text { Identifies one or more "Safety Critical" (3.2.3.10) instances that } \\
\text { the "Simulator" instance simulates }\end{array}$ \\
\hline
\end{tabular}

Original Safety-Related Concepts:

Simulator

\subsubsection{Strategy}

\section{Definiton:}

The "Strategy" concept describes an approach used to achieve a set of requirements. This approach is a design decision that relates to some category (see Kind attribute below). 
Attributes:

\begin{tabular}{|c|c|c|}
\hline Name & Description & Examples \\
\hline Kind & The kind of this strategy & $\begin{array}{l}\text { "Safety", "Reliability", } \\
\text { 'Integrity", } \\
\text { "Performance", } \\
\text { "Concurrency", } \\
\text { "Certification", "Design", } \\
\text { "Configuration", } \\
\text { "Scheduling", ... etc }\end{array}$ \\
\hline Parameters & $\begin{array}{l}\text { Specifies the strategy policy } \\
\text { parameters }\end{array}$ & $\begin{array}{l}\text { For a scheduling strategy: } \\
\text { "Round Robin", "FIFO", } \\
\text { "LIFO", ... etc }\end{array}$ \\
\hline
\end{tabular}

\section{Relationships:}

\begin{tabular}{|l|l|}
\hline Name & \multicolumn{1}{|c|}{ Description } \\
\hline Describes Design Of & $\begin{array}{l}\text { Identifies one or more "Safety Critical" (3.2.3.10) instances that is } \\
\text { designed according to a strategy described by the "Strategy" } \\
\text { instance }\end{array}$ \\
\hline
\end{tabular}

Original Safety-Related Concepts:

Safety Strategy, Scheduling Strategy

\subsubsection{Formalism}

\section{Definiton:}

The "Formalism" concept indicates that formal methods were used to develop, or prove the correctness, of some design element. 
Attributes:

\begin{tabular}{|c|c|c|}
\hline Name & Description & Examples \\
\hline Methods & $\begin{array}{l}\text { Identifies the formal methods that } \\
\text { were used }\end{array}$ & $\begin{array}{lr}\text { "Natural } & \text { Deduction", } \\
\text { "Linear } & \text { Logical } \\
\text { Framework } & \text { (LLF)", ... } \\
\text { etc } & \end{array}$ \\
\hline
\end{tabular}

Relationships:

\begin{tabular}{|l|l|}
\hline \multicolumn{1}{|c|}{ Name } & \multicolumn{1}{|c|}{ Description } \\
\hline Describes Formalism & $\begin{array}{l}\text { Identifies one or more "Safety Critical" (3.2.3.10) instances that } \\
\text { Of }\end{array}$ \\
& \begin{tabular}{l} 
"Formalism" instance \\
\hline
\end{tabular} \\
\hline
\end{tabular}

Original Safety-Related Concepts:

Formal Method

\subsubsection{Complexity}

Definiton:

The "Complexity" concept describes the complexity of a design element. Complexity aspects, such as coupling between entities or complexity of a single entity, can be measured through a variety of measures.

Attributes:

\begin{tabular}{|l|l|l|}
\hline \multicolumn{1}{|c|}{ Name } & \multicolumn{1}{|c|}{ Description } & \multicolumn{1}{c|}{ Examples } \\
\hline Measure & $\begin{array}{l}\text { Identifies the kind of the measure that } \\
\text { is used to quantify the complexity }\end{array}$ & $\begin{array}{l}\text { "Level of Nested Calls", } \\
\text { "Conditional Structures", } \\
\text { "Unconditional } \\
\end{array}$ \\
& $\begin{array}{l}\text { Branches", "Number of } \\
\text { Entry/Exit Points of } \\
\text { Code", "Big O",... etc }\end{array}$ \\
\hline
\end{tabular}




\begin{tabular}{|l|l|l|}
\hline \multicolumn{1}{|c|}{ Name } & \multicolumn{1}{|c|}{ Description } & \multicolumn{1}{|c|}{ Examples } \\
\hline Value & $\begin{array}{l}\text { An expression specifying the value, " } \mathrm{n} \text { "”, "log } \mathrm{n} ", \text { " } 25 ", \ldots \text { etc } \\
\text { or the permitted range, of the } \\
\text { measure }\end{array}$ & \\
\hline
\end{tabular}

\section{Relationships:}

\begin{tabular}{|l|l|}
\hline \multicolumn{1}{|c|}{ Name } & \multicolumn{1}{c|}{ Description } \\
\hline $\begin{array}{l}\text { Describes } \\
\text { Complexity Of }\end{array}$ & $\begin{array}{l}\text { Identifies one or more "Safety Critical" (3.2.3.10) instances for } \\
\text { which there is a measure of complexity }\end{array}$ \\
\hline
\end{tabular}

Original Safety-Related Concepts:

Complexity, Coupling

\subsubsection{Interface}

\section{Definiton:}

The "Interface" concept describes an interface between design elements. Interfaces are common between subsystems of the same system, between the system and some other external system, between software and hardware, and other situations.

Attributes:

\begin{tabular}{|l|l|l|}
\hline \multicolumn{1}{|c|}{ Name } & \multicolumn{1}{|c|}{ Description } & \multicolumn{1}{c|}{ Examples } \\
\hline $\begin{array}{l}\text { Is Between Hardware } \\
\text { And Software }\end{array}$ & $\begin{array}{l}\text { Indicates whether the interface is } \\
\text { between hardware and software }\end{array}$ & "True", "False" \\
\hline Protocol ID & Identifies the protocol used & $\begin{array}{l}\text { "MIL STD 1553" [25], } \\
\text { "Ethernet", "CORBA",... } \\
\text { etc }\end{array}$ \\
\hline $\begin{array}{l}\text { Input Function } \\
\text { Parameters }\end{array}$ & $\begin{array}{l}\text { Specifies the expected input function } \\
\text { and/or its frequency }\end{array}$ & $\begin{array}{l}\text { "Poisson with Lamba }= \\
\text { 20ms", "Periodic every 1 } \\
\text { second", ... etc }\end{array}$ \\
\hline
\end{tabular}




\begin{tabular}{|l|l|l|}
\hline \multicolumn{1}{|c|}{ Name } & \multicolumn{1}{|c|}{ Description } & \multicolumn{1}{c|}{ Examples } \\
\hline $\begin{array}{l}\text { Output Function } \\
\text { Parameters }\end{array}$ & Specifies the expected output & "Poisson with Lamba = \\
& function and/or its frequency & $\begin{array}{l}\text { 20ms", "Periodic every 1 } \\
\text { second", .. etc }\end{array}$ \\
\hline
\end{tabular}

Relationships:

\begin{tabular}{|l|l|}
\hline \multicolumn{1}{|c|}{ Name } & \multicolumn{1}{c|}{ Description } \\
\hline Is Interface For & $\begin{array}{l}\text { Identifies one or more "Safety Critical" (3.2.3.10) instances that } \\
\text { the "Interface" instance acts as an interface for }\end{array}$ \\
\hline
\end{tabular}

Original Safety-Related Concepts:

Hardware / Software Interface

\subsubsection{Concurrent}

\section{Definiton:}

The "Concurrent" concept identifies a design element that participates in a concurrency model. There are several possible roles that the design element can assume in a concurrency model, such as being a resource or software execution code that can be either active or passive. An active design element is one that is capable of generating stimuli concurrently or pseudo (seemingly) concurrently without being prompted by an explicit stimulus instance, whereas a passive one is one that cannot generate its own behaviour but only reacts when prompted by a stimulus [6].

\section{Attributes:}

\begin{tabular}{|l|l|l|}
\hline \multicolumn{1}{|c|}{ Name } & \multicolumn{1}{c|}{ Description } & \multicolumn{1}{c|}{ Examples } \\
\hline Role & The role of this entity & $\begin{array}{l}\text { "Active", "Passive", } \\
\text { "Resource" }\end{array}$ \\
\hline
\end{tabular}




\begin{tabular}{|l|l|l|}
\hline \multicolumn{1}{|c|}{ Name } & \multicolumn{1}{|c|}{ Description } & \multicolumn{1}{c|}{ Examples } \\
\hline Is Shared & $\begin{array}{l}\text { Specifies whether this entity can be } \\
\text { shared by more than one other entity } \\
\text { or not }\end{array}$ & "True", "False" \\
\hline Parameters & $\begin{array}{l}\text { Specifies how this entity acts from a } \\
\text { concurrency point of view, such as } \\
\text { the frequency of events that an active } \\
\text { entity can trigger, or the maximum } \\
\text { frequency at which a passive entity or } \\
\text { a resource can be accessed }\end{array}$ & $\begin{array}{l}\text { second", ... etc } \\
\text { sens with Lamba = }\end{array}$ \\
\hline
\end{tabular}

Relationships:

\begin{tabular}{|l|l|}
\hline \multicolumn{1}{|c|}{ Name } & \multicolumn{1}{|c|}{ Description } \\
\hline Triggers & $\begin{array}{l}\text { Identifies zero or more "Event" (3.2.3.8) instance that the } \\
\text { "Concurrent" instance triggers }\end{array}$ \\
\hline
\end{tabular}

\section{Original Safety-Related Concepts:}

Active, Passive, Shared Resource, Multi-Tasking

\subsubsection{Defensive}

\section{Definiton:}

The "Defensive" concept specifies that a design element employs a defensive design model, and describes it. In a defensive design model (e.g. defensive programming model for software), a design element checks for illegal inputs and forbid execution using illegal inputs, thus avoiding a scenario where the design element may fail due to an unfulfilled assumption on the input variables. 
Attributes:

\begin{tabular}{|c|l|l|}
\hline Name & \multicolumn{1}{|c|}{ Description } & \multicolumn{1}{c|}{ Examples } \\
\hline Defendable Inputs & $\begin{array}{l}\text { Specifies illegal input conditions that } \\
\text { this design element checks against }\end{array}$ & $\begin{array}{l}\text { "Division by Zero", } \\
\text { "Altitude }<0 ", \ldots \text { etc }\end{array}$ \\
\hline
\end{tabular}

Relationships:

\begin{tabular}{|l|l|}
\hline \multicolumn{1}{|c|}{ Name } & \multicolumn{1}{|c|}{ Description } \\
\hline Performs & $\begin{array}{l}\text { Identifies one or more "Reaction" (3.2.3.9) instances that the } \\
\text { "Defensive" instance performs to handle defendable (e.g. invalid) } \\
\text { inputs }\end{array}$ \\
\hline
\end{tabular}

Original Safety-Related Concepts:

Defensive Programming

\subsubsection{Configuration}

\section{Definiton:}

The "Configuration" concept represents a specific configuration. Software and/or

hardware configurations may change by changing memory bits, changing lookup tables, loading a software patch, and others. 
Attributes:

\begin{tabular}{|l|l|l|}
\hline \multicolumn{1}{|c|}{ Name } & \multicolumn{1}{|c|}{ Description } & \multicolumn{2}{|c|}{ Examples } \\
\hline ID & Uniquely identifies a specific & For a user interface \\
software configuration & & $\begin{array}{l}\text { software that can provide } \\
\text { interface in many } \\
\text { languages based on a } \\
\end{array}$ \\
& string lookup table: \\
& "English Interface", \\
& "French Interface", \\
& "German Interface", ... \\
& etc \\
\hline
\end{tabular}

\section{Relationships:}

None

Original Safety-Related Concepts:

Configuration

\subsubsection{Configurable}

\section{Definiton:}

The "Configurable" concept identifies a design element that can be configured or altered to produce a different configuration (3.2.3.21) or behaviour. Such change is generally performed by the user or buyer of the software, not the by vendor or its development team.

\section{Attributes:}

\begin{tabular}{|l|l|l|}
\hline \multicolumn{1}{|c|}{ Name } & \multicolumn{1}{|c|}{ Description } & \multicolumn{1}{c|}{ Examples } \\
\hline Kind & $\begin{array}{l}\text { The kind of this configurable design } \\
\text { element }\end{array}$ & $\begin{array}{l}\text { "Memory Bits", "Lookup } \\
\text { Tables", ...etc }\end{array}$ \\
\hline
\end{tabular}




\begin{tabular}{|l|l|l|}
\hline \multicolumn{1}{|c|}{ Name } & \multicolumn{1}{|c|}{ Description } & \multicolumn{1}{|c|}{ Examples } \\
\hline When & $\begin{array}{l}\text { Specifies when this configurable } \\
\text { design element can be configured to } \\
\text { change configurations }\end{array}$ & $\begin{array}{l}\text { Time", "Run-Time", ... } \\
\text { etc }\end{array}$ \\
\hline
\end{tabular}

\section{Relationships:}

\begin{tabular}{|l|l|}
\hline \multicolumn{1}{|c|}{ Name } & \multicolumn{1}{|c|}{ Description } \\
\hline Is Defaulted To & $\begin{array}{l}\text { Identifies one default "Configuration" (3.2.3.21) instance for the } \\
\text { "Configurable" instance }\end{array}$ \\
\hline Is Configurable To & $\begin{array}{l}\text { Identifies one or more "Configuration" (3.2.3.21) instances that } \\
\text { can be produced by loading loadable instances on the } \\
\text { "Configurable" instance }\end{array}$ \\
\hline
\end{tabular}

Original Safety-Related Concepts:

User Modifiable Software, Option Selectable Software

\subsubsection{Loadable}

\section{Definiton:}

The "Loadable" concept identifies a design element that can be loaded by the user

to change the configuration (3.2.3.21). Loadable design elements are loaded on configurable (3.2.3.22) design elements.

\section{Attributes:}

None

\section{Relationships:}

\begin{tabular}{|l|l|}
\hline \multicolumn{1}{|c|}{ Name } & \multicolumn{1}{|c|}{ Description } \\
\hline Is Loadable On & $\begin{array}{l}\text { Identifies one or more "Configurable" (3.2.3.22) instances on } \\
\text { which the "Loadable" instance can be loaded }\end{array}$ \\
\hline
\end{tabular}




\begin{tabular}{|l|l|}
\hline \multicolumn{1}{|c|}{ Name } & \multicolumn{1}{c|}{ Description } \\
\hline Requires & $\begin{array}{l}\text { Identifies zero or more "Configuration" (3.2.3.21) instances in } \\
\text { which the "Loadable" instance can be loaded }\end{array}$ \\
\hline Produces & $\begin{array}{l}\text { Identifies one or more "Configuration" (3.2.3.21) instances that } \\
\text { result by loading the "Loadable" instance }\end{array}$ \\
\hline
\end{tabular}

Original Safety-Related Concepts:

Field Loadable Software, Software Patch

\subsubsection{Configurator}

Definiton:

The "Configurator" concept identifies a design element that can configure configurable (3.2.3.22) design elements to change the configuration (3.2.3.21), possibly by loading loadable (3.2.3.23) design elements.

\section{Attributes:}

None

\section{Relationships:}

\begin{tabular}{|l|l|}
\hline \multicolumn{1}{|c|}{ Name } & \multicolumn{1}{c|}{ Description } \\
\hline Configures & $\begin{array}{l}\text { Identifies one or more "Configurable" (3.2.3.22) instances that can } \\
\text { be configured by the "Configurator" instance }\end{array}$ \\
\hline Loads & $\begin{array}{l}\text { Identifies one or more "Loadable" (3.2.3.23) instances that can be } \\
\text { loaded by a "Configurator" on configurable instances }\end{array}$ \\
\hline
\end{tabular}

Original Safety-Related Concepts:

Loader 


\subsubsection{Replicated}

\section{Definiton:}

The "Replicated" concept identifies a design element that participates in a replication group (3.2.3.27), such as multiple-version dissimilar software, and whose output is evaluated by a comparator (3.2.3.26).

Attributes:

\begin{tabular}{|l|l|l|}
\hline \multicolumn{1}{|c|}{ Name } & \multicolumn{1}{|c|}{ Description } & \multicolumn{1}{c|}{ Examples } \\
\hline ID & Specifies a unique identifier for this & "Filter Version 1", "Filter \\
& entity within its replication group & Version 2", "Filter \\
& & Version 3", ... etc \\
\hline
\end{tabular}

Relationships:

None

Original Safety-Related Concepts:

Multiple-Version Dissimilar Software, Software Redundancy

\subsubsection{Comparator}

\section{Definiton:}

The "Comparator" concept identifies a design element that analyzes outputs of replicated (3.2.3.25) design elements and determines the formal output of the replication group (3.2.3.27). 
Attributes:

\begin{tabular}{|c|c|c|}
\hline Name & Description & Examples \\
\hline Policy Parameters & $\begin{array}{l}\text { Specifies how the comparator } \\
\text { determines the formal output. Can } \\
\text { include assignment of weights }\end{array}$ & $\begin{array}{l}\text { "Equal Weights", } \\
\text { "Majority Voting", ... etc }\end{array}$ \\
\hline
\end{tabular}

Relationships:

\begin{tabular}{|l|l|}
\hline \multicolumn{1}{|c|}{ Name } & \multicolumn{1}{c|}{ Description } \\
\hline Compares & $\begin{array}{l}\text { Identifies two or more "Replicated" (3.2.3.25) instances whose } \\
\text { outputs are compared by the "Comparator" instance }\end{array}$ \\
\hline
\end{tabular}

Original Safety-Related Concepts:

Comparator (Voter)

\subsubsection{ReplicationGroup}

Definiton:

The "ReplicationGroup" concept identifies a software replication group composed of replicated (3.2.3.25) design elements and a comparator (3.2.3.26) that compares their outputs. For example, a replication group is an instance of software redundancy or multiple-version dissimilar software. It is a technical solution to reliability challenges and has been traditionally used in safety-critical systems.

\section{Attributes:}

\begin{tabular}{|l|l|l|}
\hline \multicolumn{1}{|c|}{ Name } & \multicolumn{1}{|c|}{ Description } & \multicolumn{1}{|c|}{ Examples } \\
\hline ID & Specifies the ID of this replication & "Radar Filter Replication \\
& group & $\begin{array}{l}\text { Group", "Controller } \\
\text { Replication Group", } \\
\end{array}$ \\
& & "REPLICATION 1", .. \\
& etc \\
\hline
\end{tabular}


Relationships:

\begin{tabular}{|lr|l|}
\hline \multicolumn{2}{|c|}{ Name } & \multicolumn{1}{c|}{ Description } \\
\hline $\begin{array}{l}\text { Owns } \\
\text { Comparator) }\end{array}$ & (with & $\begin{array}{l}\text { Identifies one "Comparator" (3.2.3.26) instance that belongs to } \\
\text { this "ReplicationGroup" instance }\end{array}$ \\
\hline $\begin{array}{l}\text { Owns } \\
\text { Replicated) }\end{array}$ & $\begin{array}{l}\text { Identifies two or more "Replicated" (3.2.3.25) instances that } \\
\text { belong to this "ReplicationGroup" instance }\end{array}$ \\
\hline
\end{tabular}

Original Safety-Related Concepts:

Multiple-Version Dissimilar Software, Software Redundancy

\subsubsection{Providing Traceability}

The refined concepts and their conceptual model satisfy all the traceabilty requirements specified in section 2.5 .

First, software requirements can be specified using the "Requirement" (3.2.3.1) concept. Then, software design elements can be traced to software requirements using the "Rationale" (3.2.3.7) concept. There also exists other concepts that allow for specialized forms of traceability to requirements, namely the "Deviation" (3.2.3.2), Nature (3.2.3.6), Partition (3.2.3.11), Implementation Style (3.2.3.4), and Behavioural Style (3.2.3.5) concepts.

In addition, the conceptual model explicitly identifies the "Event" (3.2.3.8) and "Reaction" (3.2.3.9) concepts, and establishes traceability links between those two concepts and the "Handler" concept (3.2.3.12). Furthermore, it requires reactions to be traceable to events through the "Is Consequence Of" relationship. 
It is important to note that, while the traceability described above and in section 2.5 are commonly needed for developing software, this conceptual model satisfy many more traceability requirements than those. In fact, the "Rationale" (3.2.3.7) concept is a "onesize-fits-all" traceability concept that can be used to trace any model element to any other model element or requirement. For example, the "Rationale" (3.2.3.7) concept allows traceability links from safety-critical design elements to requirements, from subsystems to requirements, and from classes to subsystems

\subsection{Information Requirements}

Section 3.1 identified detailed safety-related concepts as they appear in the airworthiness standard, which were refined in section 3.2. Therefore, a UML profile useful for safety purposes should be able to model at least this information. In other words, the refined concepts are central to defining information requirements for the definition of a UML profile, and one can transform every concept into an information requirement.

However, the information requirements we present below are defined at a level lower than the refined concept and, therefore, a refined concept may correspond to more than one information requirements. This is done so in order to break down concepts that capture many details and recognize the fact that existing profiles may only satisfy parts of a concept. For example, the "Reaction" concept and its "Consequence Of" attribute can be considered a single concept, but they capture two different pieces of information. Therefore, they are represented as two different information requirements (IREQ 22 and IREQ 23 below) to recognize the fact that a UML profile can meet only one of those two requirements (and therefore partially, but not entirely, be able to model the concept). 
Moreover, there are terms that are useful from a modeling prespective but that are not really safety-related concepts. They were not mentioned in section 3.2 because they are not concepts. Nevertheless, they help the modeler specify safety-related information in the model. Their information requirements are IREQ 1 - IREQ 8 (see below). It is common in UML profiles to provide stereotypes for such cases (see OMG UML profiles such as [5] and [6] for examples). Examples of those concepts include: Safety Context, which is used to provide high-level information about the safety of the software; Performance Context, which is used to provide high-level information about the performance aspects of the software.

The information requirements we derived from the concepts presented in section 3.2.3 are:

IREQ 1 The profile shall be able to identify a safety-related software context.

IREQ 2 The profile shall be able to identify a reliability-related software context.

IREQ 3 The profile shall be able to identify an integrity-related software context.

IREQ 4 The profile shall be able to identify a performance-related software context.

IREQ 5 The profile shall be able to identify a concurrency-related software context.

IREQ 6 The profile shall be able to identify a certification-related software context.

IREQ 7 The profile shall be able to identify a configuration-related software context.

IREQ 8 The profile shall be able to identify a design-related software context.

IREQ 9 The profile shall be able to specify software requirements, including the kind of the requirements such as safety, certification, and derived.

IREQ 10 The profile shall be able to relate software requirements to other requirements. 
IREQ 11 The profile shall be able to model a software model deviation from a plan, requirement, or a standard.

IREQ 12 The profile shall be able to model specific software implementation styles of interest to airworthiness-related software such as recursion, dynamic memory, compacted expressions, and data aliases.

IREQ 13 The profile shall be able to model time-related software such as filters.

IREQ 14 The profile shall be able to model state-related software such as state machines.

IREQ 15 The profile shall be able to model COTS software, including the rationale for using it.

IREQ 16 The profile shall be able to model previously-developed software, including the rationale for using it.

IREQ 17 The profile shall be able to model software that has deactivated code and the rationale for including the deactivated code in the design.

IREQ 18 The profile shall be able to provide traceability by relating model elements to other elements that caused related design decisions, such as relating a software comparator to a requirement element that says that a software comparator shall be used for multiple-version dissimilar software.

IREQ 19 The profile shall provide the capability to specify a reference or explanation for a modeled traceability, possibly referring to non-model elements or documents.

IREQ 20 The profile shall be able to model software events.

IREQ 21 The profile shall be able to specify how a particular software event affects the level of safety.

IREQ 22 The profile shall be able to model software reactions, or responses, to software events.

IREQ 23 The profile shall be able to specify which reactions, or responses, occur for which events.

IREQ 24 The profile shall be able to specify how a particular software reaction, or response, affects the level of safety. 
IREQ 25 The profile shall be able to model safety-critical elements.

IREQ 26 The profile shall be able to specify the criticality level of safety-critical model elements, or the element's contributions to failure conditions.

IREQ 27 The profile shall be able to model a software partition.

IREQ 28 The profile shall be able to model event handlers that perform reactions to unusual events that are detected by monitors.

IREQ 29 The profile shall be able to model software monitors.

IREQ 30 The profile shall be able to model safety monitoring software.

IREQ 31 The profile shall be able to model fault monitoring software.

IREQ 32 The profile shall be able to model integrity monitoring software.

IREQ 33 The profile shall be able to model a software simulator.

IREQ 34 The profile shall be able to specify what a software simulator simulates and the parameters by which it does so.

IREQ 35 The profile shall be able to model safety strategies.

IREQ 36 The profile shall be able to model scheduling strategies.

IREQ 37 The profile shall be able to specify the use of formal methods.

IREQ 38 The profile shall be able to model and quantify an entity's complexity on the design such as coupling and the level of code nesting.

IREQ 39 The profile shall be able to model hardware/software interfaces.

IREQ 40 The profile shall be able to describe an interface's parameters or reference external documents describing the interface parameters.

IREQ 41 The profile shall be able to model active software that can initiate a flow of control.

IREQ 42 The profile shall be able to model passive software whose execution is triggered by external events.

IREQ 43 The profile shall be able to model resources such as databases and semaphores.

IREQ 44 The profile shall be able to indicate whether a modeled resource is shared or not.

IREQ 45 The profile shall be able to distinguish software that uses defensive programming from others that do not. 
IREQ 46 The profile shall be able to describe the defensive programming parameters of software developed using defensive programming methods.

IREQ 47 The profile shall be able to model software elements whose behaviour can be modified by the user (e.g. by changing memory bits or loading look-up tables).

IREQ 48 The profile shall be able to specify what can be modified about modifiable software elements (e.g. is it a set of memory bits? A lookup table?).

IREQ 49 The profile shall be able to specify when a modifiable software element can be modified, such as at compile-time or run-time.

IREQ 50 The profile shall be able to model software that can be loaded into a system (e.g. software patch) to result in a different software configuration.

IREQ 51 The profile shall be able to model software that the user uses to change the software configuration (e.g. software used to change a memory bit or load a lookup table).

IREQ 52 The profile shall be able to model multiple-version dissimilar software.

IREQ 53 The profile shall be able to model software comparators, or voters, for multiple version dissimilar software

IREQ 54 The profile shall be able to specify the voting policy parameters for software comparators, or voters.

The requirements traceability matrix in Table 3 describes how the information requirements trace back to the original high-level requirements presented in section 2.6.3. This explains how the original-high level requirements are met by a UML profile meeting the information requirements. If a "Yes" exists in a particular cell, this means that the information requirement identified by its row traces back to the high-level requirement identified by its column. Each information requirement may trace back to more than one high-level requirement. As the table illustrates, there is an n-to-n relationship between high-level requirements and information requirements. 
The requirements traceability matrix answers the following question: Which information requirements (IREQ) are required in order to meet a particular high-level requirement (REQ)? Conversly, it can also be used to answer the following question: For a particular information requirement (IREQ), which high-level requirements (REQ) does it help meet? Therefore, it is useful if the reader is interested in knowing additional details about how a particular profile meets the original requirements (REQ). Therefore, all the information requirements trace to both the safety-related concepts, which form the basis for the information requirements, and are justified by the high-level requirements.

The rest of this document focuses on the information requirements (IREQ) rather than the high-level requirements (REQ) because they are easier to use for assessing UML profiles. Furthermore, information requirements are the true requirements that the profile must meet because they specify the particular information that must be modeled in UML models. 


\begin{tabular}{|c|c|c|c|c|c|c|c|c|}
\hline & REQ 1 & REQ 2 & REQ 3 & REQ 4 & REQ 5 & REQ 6 & REQ 7 & Total \\
\hline IREQ 1 & Yes & & Yes & & & Yes & & 3 \\
\hline IREQ 2 & Yes & & Yes & & & Yes & & 3 \\
\hline IREQ 3 & Yes & & Yes & & & Yes & & 3 \\
\hline IREQ 4 & Yes & & Yes & & & Yes & & 3 \\
\hline IREQ 5 & Yes & & Yes & & & & & 2 \\
\hline IREQ 6 & Yes & & Yes & & & & & 2 \\
\hline IREQ 7 & Yes & & Yes & & & & & 2 \\
\hline IREQ 8 & Yes & & Yes & & & & & 2 \\
\hline IREQ 9 & Yes & & Yes & Yes & & Yes & Yes & 5 \\
\hline IREQ 10 & Yes & Yes & & & Yes & Yes & Yes & 5 \\
\hline IREQ 11 & Yes & Yes & & Yes & & Yes & & 4 \\
\hline IREQ 12 & Yes & & & Yes & Yes & Yes & & 4 \\
\hline IREQ 13 & Yes & & & Yes & & Yes & & 3 \\
\hline IREQ 14 & Yes & & & Yes & & Yes & & 3 \\
\hline IREQ 15 & Yes & Yes & & Yes & Yes & Yes & & 5 \\
\hline IREQ 16 & Yes & Yes & & Yes & Yes & Yes & & 5 \\
\hline IREQ 17 & Yes & Yes & & & Yes & Yes & & 4 \\
\hline IREQ 18 & Yes & Yes & & & Yes & & & 3 \\
\hline IREQ 19 & Yes & Yes & & & Yes & Yes & & 4 \\
\hline IREQ 20 & Yes & & & & & Yes & & 2 \\
\hline IREQ 21 & Yes & & Yes & & Yes & Yes & & 4 \\
\hline IREQ 22 & Yes & & & Yes & & Yes & & 3 \\
\hline IREQ 23 & Yes & & & Yes & & Yes & & 3 \\
\hline IREQ 24 & Yes & & Yes & Yes & Yes & Yes & & 5 \\
\hline IREQ 25 & Yes & & Yes & & & Yes & & 3 \\
\hline IREQ 26 & Yes & & Yes & & Yes & Yes & & 4 \\
\hline IREQ 27 & Yes & & & Yes & & Yes & & 3 \\
\hline IREQ 28 & Yes & & & Yes & & Yes & & 3 \\
\hline IREQ 29 & Yes & & & Yes & & Yes & & 3 \\
\hline IREQ 30 & Yes & & Yes & Yes & & Yes & & 4 \\
\hline IREQ 31 & Yes & & Yes & Yes & & Yes & & 4 \\
\hline IREQ 32 & Yes & & Yes & Yes & & & & 3 \\
\hline IREQ 33 & Yes & & & & & Yes & & 2 \\
\hline IREQ 34 & Yes & & & & & Yes & & 2 \\
\hline IREQ 35 & Yes & & Yes & Yes & & Yes & & 4 \\
\hline IREQ 36 & Yes & & Yes & Yes & & & & 3 \\
\hline IREQ 37 & Yes & & & & Yes & & & 2 \\
\hline IREQ 38 & Yes & & & Yes & & & & 2 \\
\hline IREQ 39 & Yes & & & & & Yes & & 2 \\
\hline IREQ 40 & Yes & Yes & & & & & & 2 \\
\hline IREQ 41 & Yes & & & Yes & & & & 2 \\
\hline IREQ 42 & Yes & & & Yes & & & & 2 \\
\hline IREQ 43 & Yes & & & Yes & & & & 2 \\
\hline IREQ 44 & Yes & & & Yes & & & & 2 \\
\hline IREQ 45 & Yes & & & Yes & Yes & & & 3 \\
\hline IREQ 46 & Yes & & & Yes & Yes & & & 3 \\
\hline IREQ 47 & Yes & & & Yes & Yes & Yes & & 4 \\
\hline IREQ 48 & Yes & & & Yes & Yes & Yes & & 4 \\
\hline IREQ 49 & Yes & & & Yes & Yes & & & 3 \\
\hline IREQ 50 & Yes & & & Yes & Yes & & & 3 \\
\hline IREQ 51 & Yes & & & Yes & Yes & & & 3 \\
\hline IREQ 52 & Yes & & & Yes & & Yes & & 3 \\
\hline
\end{tabular}




\begin{tabular}{|c|c|c|c|c|c|c|c|c|}
\hline & REQ 1 & REQ 2 & REQ 3 & REQ 4 & REQ 5 & REQ 6 & REQ 7 & Total \\
\hline IREQ 53 & Yes & & & Yes & & Yes & & 3 \\
\hline IREQ 54 & Yes & & & Yes & & & & 2 \\
\hline Total & 54 & 8 & 18 & 33 & 18 & 34 & 2 & 167 \\
\hline
\end{tabular}

Table 3: Tracing information requirements to the original high-level requirements. 


\section{EXISTING UML PROFILES}

This section introduces existing UML profiles and approaches and evaluates each one of them with respect to the information requirements identified in section 3.3 . This is required to determine whether a suitable profile already exists or not, which would determine whether an existing profile should be extended (if necessary) or a completely new profile should be defined. Details of the evaluations are reported in Appendix $\mathrm{F}$ and summarized in the following sections.

\subsection{Quality of Service and Fault Tolerance OMG Profile}

OMG released a profile to model Quality of Service (QoS) for high-quality and FaultTolerant (FT) systems. The profile, presented in [5], includes frameworks to describe quality of service, risk assessment, and fault tolerance.

The framework to describe quality of service includes mechanisms to describe generic quality of service driven from quality-based requirements. It is not specific to any kind of quality of service, such as safety. Its mechanisms focus on characteristics, constraints, and levels of quality of service. The risk assessment framework includes support for model-based risk assessment. It provides mechanisms for modeling risk contexts, stakeholders, assets, strengths, weaknesses, opportunities and threats, unwanted incidents, risk quantification, and risk mitigation and treatments. The fault tolerance framework includes mechanisms for describing fault-tolerant software architectures in general as a technical solution to reliability requirements. It focuses on modeling software redundancy, or software replication. 
Table 18 in Appendix F presents an analysis of the profile with respect to each of the information requirements described in section 3.3. The table concludes that this profile is not adequate for extension to meet the information requirements since only 17 of the information requirements (out of 54) are fulfilled.

\subsection{Schedulability, Performance, and Time OMG Profile}

OMG released a profile, the Schedulability, Performance, and Time (SPT) profile, which provides mechanisms to model concepts of importance to real-time systems. Real-time systems are those where there exist timing requirements on when the responses to events occur. Soft real-time systems are those were late responses may be acceptable if they are not within a specified range, whereas hard real-time systems are those where late responses are unacceptable and may be fatal [6]. The profile, presented in [6], includes frameworks to model resources, time, concurrency, schedulability, performance, and CORBA schedulability properties. Using it allows developers to perform performance analysis of the model.

The resource modeling framework includes mechanisms to model resources, components that acquire and release them, and their deployment on hosts. The time modeling framework includes mechanisms to model clocks, timers, timeouts, and actions that are applied on them such as delays, interrupts, events, pause, reset, start, and stop. The concurrency modeling framework includes mechanisms to model synchronous and asynchronous actions, and event queues for immediate and deferred event processing. The schedulability analysis framework includes mechanism to model actions, engines, responses, scheduling resources, triggers, action schedulers, and scheduling hosts. The 
performance analysis framework includes mechanism to model performance contexts, open and closed workloads, and steps. The CORBA schedulability framework includes mechanisms to model CORBA channels, connections, clients, servers, and Object Resource Brokers (ORBs).

Table 19 in Appendix F presents an analysis of the profile with respect to each of the information requirements described in section 3.3. The table concludes that this profile is not adequate for extension to meet the information requirements since only 6 of the information requirements (out of 54) are fulfilled. The profile does not meet many of the information requirements because it does not cover safety and reliabity topics.

\subsection{HIDOORS Profile}

The High Integrity Distributed Object-Oriented Real-Time Systems (HIDOORS) was a joint research project by several European companies and research institutions. One of the goals of HIDOORS was to introduce mechanisms for modeling safety-critical and embedded real-time applications. Those mechanisms were required to be compliant with OMG's SPT profile (see section 4.2), provide mechanisms for modeling the Rate Monotonic Analysis (RMA) scheduling strategy, and provide specific concepts relating to inter-task communication. The researchers involved in this project felt that UML's SPT profile was too general and too fundamental to provide mechanisms for specifying RMA and some inter-task communication concepts [26]. The profile therefore specializes some SPT concepts such as triggers, actions, resources, and scheduling jobs. Furthermore, it provides mechanisms to model inter-task communication styles such as buffers, black boards, and events. 
Table 20 in Appendix F presents an analysis of the profile with respect to each of the information requirements described in section 3.3. The table concludes that this profile is not adequate for extension to meet the information requirements since only 6 of the information requirements (out of 54) are fulfilled. The profile included only few stereotypes so it does not meet most of the information requirements. In fact, it did not meet any information requirements that were not already met by the SPT profile.

\subsection{Effects of Message Loss, Delay, and Corruption}

Jan Jürjens presented a UML profile in [27] that aimes at addressing safety issues from a fault-tolerant point of view. Jürjens argued that safety goals are often expressed quantitatively via the maximum failure rate, and then presented some possible failures that served as the basis of the proposed UML profile. Thus, his profile assumes that those failures are based on the concept of transmitting messages on links and between nodes. The profile included mechanisms to model risks, crashes, guarantees, redundancy, safe links, safe dependencies, safety critical elements, safe behaviours, containment, and error handling.

Table 21 in Appendix F presents an analysis of the profile with respect to each of the information requirements described in section 3.3. The table concludes that this profile is not adequate for extension to meet the information requirements since only 7 of the information requirements (out of 54) are fulfilled. In fact, it does not meet many of the information requirements because assuming the airworthiness standard does not make the assumption that unsafe states result from failures of transmitting messages because safety is a bigger issue than that. 


\subsection{Patterns for Reliability and Safety}

Hansen and Gullesen presented in [28] a series of UML patterns that can be used to model some aspects of safety-critical systems. They presented patterns for modeling safety quality of service, software diversity and voting, partial diversity with built-in diagnostic or monitoring, "safe" communication protocols, and some other topics such as testing, hazard analysis and quality development. Their work was driven by the IEC 61508 standard in [24]. They have therefore used the concept of Safety Integrity Level (SIL), which is similar to the concept of software level presented in the airworthiness standard [4]. The patterns include mechanisms to model the SIL levels, and other patterns that could be used to explicitly model, in use cases, redundancy, monitoring, and voting based on multiple output comparisons.

Table 22 in Appendix F presents an analysis of the profile with respect to each of the information requirements described in section 3.3. The table concludes that the patterns presented in this paper are not adequate for extension to meet the information requirements since only 1 of the information requirements (out of 54) are fulfilled. The patterns mostly focus on reliability and software replication issues, whereas the information requirements cover a bigger concern.

\subsection{Summary}

Table 23 in Appendix F summarizes how each of those existing UML profiles scores with respect to addressing the information requirements identified in section 3.3. Each 
profile's score is calculated based on how many information requirements it meets. Therefore, the maximum score is $54(100 \%)$.

As it can be noticed from previous sections, which results are summarized in Table 23, none of the existing profiles that were evaluated achieves more than $31 \%$ of the maximum score. In fact, all of the profiles combined only meet $44 \%$ of the information requirements.

The two OMG profiles are useful, but only within their intended domain. The OMG QoS and FT profile is suitable for modeling fault tolerance and software redundancy. The OMG SPT profile is suitable for modeling schedulability, performance, and concurrency concepts. However, it was evident in section 3 that safety and airworthiness are dependent on many domains. Therefore, those two OMG profiles would be complimentary, but not complete enough. It should also be noticed that the SPT profile included more details than the QoS and FT profile, but it was centred on domains that are somehow less important to safety (resources, time, concurrency, performance, and schedulability) than those covered by the QoS and FT profile (quality of service, risk assessment, fault tolerance).

Furthermore, the profile for the "effects of messages on safety" introduces some useful stereotypes such as $\quad<$ safe behaviour $>>, \quad<$ guarantee $>>, \quad<$ critical $>$, $<<$ containment $>>$ and $<<$ redundancy $>>$. However, some of them are too general to be effective, such as <<critical $>$ which does not tell us the criticality level (e.g. airworthiness level) of the software. Neverthless, it meets some information requirements that are not met by the 2 OMG profiles such as the ability to model exception handlers. 
The "HIDOORS" profile did not meet any information requirements that were not already met by the SPT profile.

The "Patterns" paper did not fulfil any information requirements that were not fulfilled by the other profiles.

Because none of the existing UML profiles and patterns comes close to fulfilling the information requirements, a new UML profile is proposed instead of extending an existing one. This has the advantage that the resultant profile will be coherent and specifically designed to meet the information requirements instead of "patching" an existing profile that was originally designed for some other purpose. 


\section{PROPOSED UML PROFILE}

Since no suitable UML profile was found, this section introduces a new UML profile that meets all of the information requirements. Section 5.1 describes the template we use to describe the profile whereas section 5.2 describes the profile proper. Examples are then presented in section 5.3 to explain in detail how to use this proposed UML profile. Section 5.4 suggests a methodology to be followed when using the profile.

\subsection{UML Profile-Template Description}

This section introduces a UML profile that satisfies all of the information requirements specified in section 3.3. The discussion on how this proposed profile meets all of the information requirements is presented in section 8.1.

Each of the subsections of section 5.2 describes a single stereotype and associated tagged values. Most of the stereotypes correspond to some refined concepts. However, some additional stereotypes, which do not correspond to any refined concepts, were deemed helpful for modeling purposes are introduced. The stereotypes' tagged values correspond to the refined concepts' attributes and relationships. The following information is presented for each stereotype:

1. Definition: This presents a definition for the stereotype. It describes the stereotype and gives its general purpose.

2. Related Concept: This identifies, if applicable, the concept from section 3.2 .2 that the stereotype represents. 
3. Base Classes: This lists the UML meta-classes on which the stereotype may be applied. The explanation on how and why the stereotype may be applied on each meta-class is also presented. This does not identify meta-classes on which the stereotype may not be applied to allow future extenstions of this profile to be backwards compatible. This is because future extension may permit the application of the stereotypes on meta-classes that are not explicit here. This is necessary because extending UML, such as in the form of UML profiles, permits adding new rules but does not allow removing existing rules.

4. Tags: This lists the tags that the stereotype has. For each tag, the type, multiplicity, and a description is presented.

The base classes of each stereotype are presented in a table. For example, the base classes of the $<<$ SafetyCritical $>>(5.2 .17)$ stereotype are presented as follows:

\begin{tabular}{|l|l|}
\hline \multicolumn{1}{|c|}{ Base Class } & \multicolumn{1}{c|}{ Usage Rationale } \\
\hline Class & $\begin{array}{l}\text { To indicate that the class is safety-critical (3.2.3.10) and specify } \\
\text { its safety (e.g. airworthiness) level }\end{array}$ \\
\hline Operation & $\begin{array}{l}\text { To indicate that invoking the operation is safety-critical (3.2.3.10) } \\
\text { and specify its safety (e.g. airworthiness) level }\end{array}$ \\
\hline Relationship & $\begin{array}{l}\text { To indicate that the relationship is safety-critical (3.2.3.10) and } \\
\text { specify its safety (e.g. airworthiness) level }\end{array}$ \\
\hline
\end{tabular}

The first column lists the UML meta-classes on whose instances the stereotype can be applied. The UML classes are specified as defined in the UML meta-model in [29] and [30]. This profile uses only the following base classes in the first column: 
1. Collaboration: Used to represent instances of class “CompositeStructures::Collaborations::Collaboration”.

2. Class: Used to represent instances of class "Kernel::Class" and class "BasicComponents::Component".

3. Operation: Used to represent instances of class "Kernel::Operation".

4. Relationship: Used to represent instances of class "Kernel::Relationship".

The second column describes why the stereotype can be applied on each base class.

Thus, the above table is read as follows: The $<<$ SafetyCritical $>>$ stereotype can be applied on all UML model classes that are instances of the following meta-classes (first column): Class, Operation, and Relationship. It is applied on each of the base classes as follows (second column):

1. Class: To indicate that the class is safety-critical (3.2.3.10) and specify its safety (e.g. airworthiness) level

2. Operation: To indicate that invoking the operation is safety-critical (3.2.3.10) and specify its safety (e.g. airworthiness) level

3. Relationship: To indicate that the relationship is safety-critical (3.2.3.10) and specify its safety (e.g. airworthiness) level

The tags of each stereotype are presented in a table. For example, the tags of the $<<$ SafetyCritical $>>(5.2 .17)$ stereotype are presented as follows: 


\begin{tabular}{|l|l|l|l|}
\hline \multicolumn{1}{|c|}{ Name } & \multicolumn{1}{|c|}{ Type } & Multiplicity & \multicolumn{1}{c|}{ Description } \\
\hline CriticalityLevel & Enumeration & {$[0 . .1]$} & See attribute: Criticality Level \\
\hline ConfidenceLevel & Enumeration & {$[0 . .1]$} & $\begin{array}{l}\text { See attribute: Confidence } \\
\text { Level }\end{array}$ \\
\hline TriggeredEvent & $\begin{array}{l}\text { Reference to a } \\
\text { model element } \\
\text { stereotyped } \\
<<\text { Event }>>\end{array}$ & $\begin{array}{l}\text { See relationship: Triggers } \\
\text { (5.2.15) (or its } \\
\text { subclass } \\
<<\text { Reaction>> } \\
(5.2 .16))\end{array}$ & \\
\hline
\end{tabular}

The first column specifies the tag name, the second column specifies the type of the tag, the third column specifies its multiplicity, and the fourth column provides a description of the tag. In most cases, the fourth column will refer the reader to an attribute or a relationship of the related concept.

This example tells us that this stereotype has the following tags and they are described as follows:

1. The "CriticalityLevel" tag (first column) of the $<<$ SafetyCritical $>>$ stereotype is specified through an enumeration (second column). It can be specified zero or one time (third column) for each stereotype. Its description is the same as the "Criticality Level" attribute of the related concept ("Safety Critical" (3.2.3.10) concept in this case) (fourth column).

2. The "ConfidenceLevel" tag (first column) of the $<<$ SafetyCritical $>>$ stereotype is specified through an enumeration (second column). It can be specified zero or one 
time (third column) for each stereotype. Its description is the same as the "Confidence Level" attribute of the related concept ("Safety Critical" (3.2.3.10) concept in this case) (fourth column).

3. The "TriggeredEvent" tag (first column) of the $<<$ SafetyCritical $>>$ stereotype is specified through a reference to a model element stereotyped with $<<$ Event $\gg>$ or $<<$ Reaction $\gg>$ (second column). It can be specified zero or as many times as one wishes (third column) for each stereotype. Its description is the same as the "Triggers" relationship of the related concept ("SafetyCritical" (3.2.3.10) concept in this case) (fourth column).

\subsection{Profile Description}

\subsection{1 《<SafetyContext $\gg$}

\section{Definiton:}

The $<<$ SafetyContext $>$ stereotype is used to indicate that there is safety-related information of interest such as information representing the original primarily safety concepts listed in Appendix C.1.

\section{Related Concept:}

None

Base Classes:

\begin{tabular}{|l|l|}
\hline \multicolumn{1}{|c|}{ Base Class } & \multicolumn{1}{c|}{ Usage Rationale } \\
\hline Collaboration & To indicate that the collaboration contains safety information \\
\hline
\end{tabular}

Tags:

None 


\subsection{2 <<ReliabilityContext $>>$}

\section{Definiton:}

The $<<$ ReliabilityContext $\gg>$ stereotype is used to indicate that there is reliabilityrelated information of interest such as information representing the original primarily reliability concepts listed in Appendix C.2. In one specific usage, it could also be used to describe or identify a specific replication group (see related concept).

\section{Related Concept:}

ReplicationGroup (3.2.3.27)

Base Classes:

\begin{tabular}{|l|l|}
\hline \multicolumn{1}{|c|}{ Base Class } & \multicolumn{1}{|c|}{ Usage Rationale } \\
\hline Collaboration & $\begin{array}{l}\text { To indicate that the collaboration contains reliability information, } \\
\text { or to identify a particular replication group (3.2.3.27) composed } \\
\text { of replicated (3.2.3.25) design elements and a comparator } \\
(3.2 .3 .26)\end{array}$ \\
\hline
\end{tabular}

Tags:

\begin{tabular}{|l|l|l|l|}
\hline \multicolumn{1}{|c|}{ Name } & \multicolumn{1}{|c|}{ Type } & Multiplicity & \multicolumn{1}{c|}{ Description } \\
\hline ID & String & {$[0 . .1]$} & See attribute: ID \\
\hline
\end{tabular}

\subsection{3 《<IntegrityContext >}

\section{Definiton:}

The $<<$ IntegrityContext $>>$ stereotype is used to indicate that there is safety-related information of interest such as information representing the original primarily integrity concepts listed in Appendix C.3. 
Related Concept:

None

Base Classes:

\begin{tabular}{|l|l|}
\hline \multicolumn{1}{|c|}{ Base Class } & \multicolumn{1}{c|}{ Usage Rationale } \\
\hline Collaboration & To indicate that the collaboration contains integrity information \\
\hline
\end{tabular}

Tags:

None

\subsection{4 $<<$ PerformanceContext $>>$}

Definiton:

The $<<$ PerformanceContext $>>$ stereotype is used to indicate that there is performance-related information of interest such as information representing the original primarily performance concepts listed in Appendix C.4.

\section{Related Concept:}

None

Base Classes:

\begin{tabular}{|l|l|}
\hline \multicolumn{1}{|c|}{ Base Class } & \multicolumn{1}{|c|}{ Usage Rationale } \\
\hline Collaboration & $\begin{array}{l}\text { To indicate that the collaboration contains performance } \\
\text { information }\end{array}$ \\
\hline
\end{tabular}

Tags:

None 


\subsection{5 $<<$ ConcurrencyContext $>>$}

\section{Definiton:}

The $<<$ ConcurrencyContext $>>$ stereotype is used to indicate that there is concurrency-related information of interest such as information representing the original primarily concurrency concepts listed in Appendix C.5.

\section{Related Concept:}

None

Base Classes:

\begin{tabular}{|l|l|}
\hline \multicolumn{1}{|c|}{ Base Class } & \multicolumn{1}{|c|}{ Usage Rationale } \\
\hline Collaboration & $\begin{array}{l}\text { To indicate that the collaboration contains concurrency } \\
\text { information }\end{array}$ \\
\hline
\end{tabular}

Tags:

None

\subsection{6 $<<$ CertificationContext $>>$}

Definiton:

The $<<$ CertificationContext $>>$ stereotype is used to indicate that there is certification-related information of interest such as information representing the original primarily certification concepts listed in Appendix C.6.

\section{Related Concept:}

None 
Base Classes:

\begin{tabular}{|l|l|}
\hline \multicolumn{1}{|c|}{ Base Class } & \multicolumn{1}{|c|}{ Usage Rationale } \\
\hline Collaboration & $\begin{array}{l}\text { To indicate that the collaboration contains certification } \\
\text { information }\end{array}$ \\
\hline
\end{tabular}

Tags:

None

\subsection{7 <-DesignContext $>$}

Definiton:

The $<<$ DesignContext $\gg>$ stereotype is used to indicate that there is specific designrelated information of interest such as information representing the original primarily design concepts listed in Appendix C.7.

Related Concept:

None

Base Classes:

\begin{tabular}{|l|l|}
\hline \multicolumn{1}{|c|}{ Base Class } & \multicolumn{1}{|c|}{ Usage Rationale } \\
\hline Collaboration & $\begin{array}{l}\text { To indicate that the collaboration contains specific design } \\
\text { information such as design constraints and design decisions }\end{array}$ \\
\hline
\end{tabular}

Tags:

None

\subsection{8 $<<$ ConfigurationContext $>>$}

Definiton:

The $<<$ ConfigurationContext $>>$ stereotype is used to indicate that there is configuration-related information of interest such as information representing the 
original primarily configuration concepts listed in Appendix C.8. In one specific usage, it could also be used to describe or identify a specific configuration (see related concept).

\section{Related Concept:}

Configuration (3.2.3.21)

Base Classes:

\begin{tabular}{|l|l|}
\hline \multicolumn{1}{|c|}{ Base Class } & \multicolumn{1}{|c|}{ Usage Rationale } \\
\hline Collaboration & To indicate that the collaboration contains configuration \\
& $(3.2 .3 .21)$ information, or to identify a particular configuration \\
& $(3.2 .3 .21)$ \\
\hline
\end{tabular}

Tags: (refer to section 3.2.3.21 for details)

\begin{tabular}{|l|l|l|l|}
\hline \multicolumn{1}{|c|}{ Name } & \multicolumn{1}{|c|}{ Type } & Multiplicity & \multicolumn{1}{c|}{ Description } \\
\hline ID & String & {$[0 . .1]$} & See attribute: ID \\
\hline
\end{tabular}

\subsection{9 <<Requirement $>$}

Definiton:

See related concept.

\section{Related Concept:}

Requirement (3.2.3.1)

Base Classes:

\begin{tabular}{|l|l|}
\hline \multicolumn{1}{|c|}{ Base Class } & \multicolumn{1}{c|}{ Usage Rationale } \\
\hline Collaboration & $\begin{array}{l}\text { To specify a requirement that the design in the collaboration } \\
\text { fulfills }\end{array}$ \\
\hline Class & To specify a requirement that the class fulfills \\
\hline Operation & To specify a requirement that the operation fulfills \\
\hline
\end{tabular}




\begin{tabular}{|l|l|}
\hline \multicolumn{1}{|c|}{ Base Class } & \multicolumn{1}{c|}{ Usage Rationale } \\
\hline Relationship & To specify a requirement that a relationship fulfills \\
\hline
\end{tabular}

Tags: (refer to section 3.2.3.1 for details)

\begin{tabular}{|l|l|l|ll|}
\hline \multicolumn{1}{|c|}{ Name } & \multicolumn{1}{|c|}{ Type } & Multiplicity & \multicolumn{1}{c|}{ Description } \\
\hline ID & String & {$[0 . .1]$} & See attribute: ID \\
\hline Kind & Enumeration & {$[0 . .1]$} & See attribute: Kind \\
\hline Specification & Expression & {$[1 . .1]$} & See attribute: Specification \\
\hline OfRequirement & $\begin{array}{l}\text { Reference to a } \\
\text { model element } \\
\text { stereotyped } \\
<<\text { Requirement }>> \\
(5.2 .9)\end{array}$ & $\begin{array}{l}\text { See relationship: Is } \\
\text { Requirement Of }\end{array}$ \\
\hline
\end{tabular}

\subsubsection{0 <<Deviation >>}

\section{Definiton:}

See related concept.

\section{Related Concept:}

Deviation (3.2.3.2)

Base Classes:

\begin{tabular}{|l|l|}
\hline \multicolumn{1}{|c|}{ Base Class } & \multicolumn{1}{c|}{ Usage Rationale } \\
\hline Collaboration & $\begin{array}{l}\text { To specify that the collaboration's design and/or implementation } \\
\text { deviates from a requirement (3.2.3.1), standard, or plan }\end{array}$ \\
\hline Class & $\begin{array}{l}\text { To specify that the class' design and/or implementation deviates } \\
\text { from a requirement (3.2.3.1), standard, or plan }\end{array}$ \\
\hline Operation & $\begin{array}{l}\text { To specify that the operation's design and/or implementation } \\
\text { deviates from a requirement (3.2.3.1), standard, or plan }\end{array}$ \\
\hline
\end{tabular}




\begin{tabular}{|l|l|}
\hline \multicolumn{1}{|c|}{ Base Class } & \multicolumn{1}{c|}{ Usage Rationale } \\
\hline Relationship & $\begin{array}{l}\text { To specify that the relationship's design and/or implementation } \\
\text { deviates from a requirement (3.2.3.1), standard, or plan }\end{array}$ \\
\hline
\end{tabular}

Tags: (refer to section 3.2.3.2 for details)

\begin{tabular}{|l|l|l|l|}
\hline \multicolumn{1}{|c|}{ Name } & \multicolumn{1}{|c|}{ Type } & Multiplicity & \multicolumn{1}{c|}{ Description } \\
\hline Kind & Enumeration & {$[0 . .1]$} & See attribute: Kind \\
\hline Reference & $\begin{array}{l}\text { Reference to a } \\
\text { model element } \\
\text { stereotyped } \\
\text { <<Requirement }>> \\
(5.2 .9)\end{array}$ & & See relationship: References \\
\hline Explanation & String & {$\left[0 . .^{*}\right]$} & See attribute: Explanation \\
\hline
\end{tabular}

\subsubsection{1 《<ImplementationStyle»>}

\section{Definiton:}

See related concept.

\section{Related Concept:}

ImplementationStyle (3.2.3.4)

Base Classes:

\begin{tabular}{|l|l|}
\hline \multicolumn{1}{|c|}{ Base Class } & \multicolumn{1}{c|}{ Usage Rationale } \\
\hline Class & $\begin{array}{l}\text { To identify an implementation style (3.2.3.4) (e.g. dynamic } \\
\text { memory, recursive algorithms, .. etc) that is followed in the } \\
\text { implementation of the class }\end{array}$ \\
\hline Operation & $\begin{array}{l}\text { To identify an implementation style (3.2.3.4) (e.g. dynamic } \\
\text { memory, recursive algorithms, ... etc) that is followed in the } \\
\text { implementation of the operation }\end{array}$ \\
\hline
\end{tabular}


Tags: (refer to section 3.2.3.4 for details)

\begin{tabular}{|l|l|l|l|}
\hline \multicolumn{1}{|c|}{ Name } & \multicolumn{1}{|c|}{ Type } & Multiplicity & \multicolumn{1}{c|}{ Description } \\
\hline Kind & Enumeration & {$[0 . .1]$} & See attribute: Kind \\
\hline Parameter & Expression & {$\left[0 . *^{*}\right]$} & See attribute: Parameters \\
\hline Reference & $\begin{array}{l}\text { Reference to a } \\
\text { model element } \\
\text { stereotyped } \\
\text { <<equirement>> } \\
(5.2 .9), \text { or a String }\end{array}$ & {$\left[0 .{ }^{*}\right]$} & See relationship: References \\
\hline Explanation & String & {$\left[0 .{ }^{*}\right]$} & See attribute: Explanation \\
\hline
\end{tabular}

\subsubsection{2 < BehaviouralStyle $>$}

\section{Definiton:}

See related concept.

\section{Related Concept:}

BehaviouralStyle (3.2.3.5)

Base Classes:

\begin{tabular}{|l|l|}
\hline \multicolumn{1}{|c|}{ Base Class } & \multicolumn{1}{c|}{ Usage Rationale } \\
\hline Collaboration & $\begin{array}{l}\text { To identify a behavioural style (3.2.3.5) (e.g. state-related such as } \\
\text { state machines, ... etc) that is followed in the implementation of } \\
\text { the design specified in the collaboration }\end{array}$ \\
\hline Class & $\begin{array}{l}\text { To identify a behavioural style (3.2.3.5) (e.g. state-related as in } \\
\text { class attributes, time-related as in filters, ... etc) that is followed } \\
\text { in the implementation of the class }\end{array}$ \\
\hline Operation & $\begin{array}{l}\text { To identify a behavioural style (3.2.3.5) (e.g. state-related as in } \\
\text { static operations, time-related as in filters) that is followed in the } \\
\text { implementation of the operation }\end{array}$ \\
\hline
\end{tabular}


Tags: (refer to section 3.2.3.5 for details)

\begin{tabular}{|c|c|c|c|}
\hline Name & Type & Multiplicity & Description \\
\hline Kind & Enumeration & {$[0 . .1]$} & See attribute: Kind \\
\hline Parameter & Expression & {$\left[0 .{ }^{*}\right]$} & See attribute: Parameters \\
\hline Reference & $\begin{array}{l}\text { Reference to a } \\
\text { model element } \\
\text { stereotyped } \\
<<\text { Requirement }> \\
(5.2 .9) \text {, or a String }\end{array}$ & {$\left[0 . .^{*}\right]$} & See relationship: References \\
\hline Explanation & String & {$\left[0 .{ }^{*}\right]$} & See attribute: Explanation \\
\hline
\end{tabular}

\subsubsection{3 $<<$ Nature $>>$}

Definiton:

See related concept.

Related Concept:

Nature (3.2.3.6)

Base Classes:

\begin{tabular}{|l|l|}
\hline \multicolumn{1}{|c|}{ Base Class } & \multicolumn{1}{c|}{ Usage Rationale } \\
\hline Class & $\begin{array}{l}\text { To identify the nature (3.2.3.6) (e.g. COTS, previously developed, } \\
\text { deactivated, ... etc) of the class }\end{array}$ \\
\hline Operation & $\begin{array}{l}\text { To identify the nature (3.2.3.6) (e.g. deactivated, ... etc) of the } \\
\text { operation }\end{array}$ \\
\hline
\end{tabular}

Tags: (refer to section 3.2.3.6 for details)

\begin{tabular}{|l|l|l|l|}
\hline \multicolumn{1}{|c|}{ Name } & \multicolumn{1}{c|}{ Type } & Multiplicity & \multicolumn{1}{c|}{ Description } \\
\hline Kind & Enumeration & {$[0 . .1]$} & See attribute: Kind \\
\hline
\end{tabular}




\begin{tabular}{|l|l|l|l|}
\hline \multicolumn{1}{|c|}{ Name } & \multicolumn{1}{|c|}{ Type } & Multiplicity & \multicolumn{1}{c|}{ Description } \\
\hline Reference & $\begin{array}{l}\text { Reference to a } \\
\text { model element } \\
\text { stereotyped } \\
\text { <<Requirement }> \\
(5.2 .9), \text { or a String }\end{array}$ & & See relationship: References \\
\hline Explanation & String & {$\left[0 . .^{*}\right]$} & See attribute: Explanation \\
\hline
\end{tabular}

\subsubsection{4 <<Rationale $>$}

\section{Definiton:}

See related concept.

\section{Related Concept:}

Rationale (3.2.3.7)

Base Classes:

\begin{tabular}{|l|l|}
\hline \multicolumn{1}{|c|}{ Base Class } & \multicolumn{1}{c|}{ Usage Rationale } \\
\hline Collaboration & $\begin{array}{l}\text { To explain the rationale (3.2.3.7) or explain the design decisions } \\
\text { for the design specified in the collaboration }\end{array}$ \\
\hline Class & $\begin{array}{l}\text { To explain the rationale (3.2.3.7), explain the design decisions, or } \\
\text { identify the reason for defining and developing the class }\end{array}$ \\
\hline Operation & $\begin{array}{l}\text { To explain the rationale (3.2.3.7), explain the design decisions, or } \\
\text { identify the reason for defining and developing the operation }\end{array}$ \\
\hline Relationship & $\begin{array}{l}\text { To explain the rationale (3.2.3.7), explain the design decisions, or } \\
\text { identify the reason for defining the relationship }\end{array}$ \\
\hline
\end{tabular}


Tags: (refer to section 3.2.3.7 for details)

\begin{tabular}{|l|l|l|l|}
\hline \multicolumn{1}{|c|}{ Name } & \multicolumn{1}{|c|}{ Type } & Multiplicity & \multicolumn{1}{c|}{ Description } \\
\hline Reference & $\begin{array}{l}\text { Reference to a } \\
\text { model element } \\
\text { stereotyped } \\
<<\text { Requirement }>> \\
\text { (5.2.9), or a String }\end{array}$ & & See relationship: References \\
\hline Explanation & String & {$\left[0 .{ }^{*}\right]$} & See attribute: Explanation \\
\hline
\end{tabular}

\subsubsection{5 $<<$ Event $>>$}

\section{Definiton:}

See related concept.

\section{Related Concept:}

Event (3.2.3.8)

Base Classes:

\begin{tabular}{|l|l|}
\hline \multicolumn{1}{|c|}{ Base Class } & \multicolumn{1}{c|}{ Usage Rationale } \\
\hline Class & $\begin{array}{l}\text { To indicate that the class contains information that constitutes and } \\
\text { describes an event (3.2.3.8) of interest }\end{array}$ \\
\hline Operation & $\begin{array}{l}\text { To indicate that invoking the specified operation is an event } \\
(3.2 .3 .8) \text { of interest, and to further describe the operation's effect } \\
\text { on safety }\end{array}$ \\
\hline
\end{tabular}

Tags: (refer to section 3.2.3.8 for details)

\begin{tabular}{|l|l|l|l|}
\hline \multicolumn{1}{|c|}{ Name } & \multicolumn{1}{|c|}{ Type } & Multiplicity & \multicolumn{1}{c|}{ Description } \\
\hline Kind & Enumeration & {$[0 . .1]$} & See attribute: Kind \\
\hline When & Expression & {$\left[0 . *^{*}\right]$} & See attribute: When \\
\hline EffectOnSafetyDirection & Enumeration & {$\left[0 . .^{*}\right]$} & $\begin{array}{l}\text { See attribute: Effect On } \\
\text { Safety Direction }\end{array}$ \\
\hline
\end{tabular}




\begin{tabular}{|l|l|l|l|}
\hline \multicolumn{1}{|c|}{ Name } & \multicolumn{1}{|c|}{ Type } & Multiplicity & \multicolumn{1}{|c|}{ Description } \\
\hline EffectOnSafetyValue & Expression & {$\left[0 . .^{*}\right]$} & $\begin{array}{l}\text { See attribute: Effect On } \\
\text { Safety Value }\end{array}$ \\
\hline EffectOnSafetyContext & Expression & {$\left[0 .{ }^{*}\right]$} & $\begin{array}{l}\text { See attribute: } \\
\text { EffectOnSafetyContext }\end{array}$ \\
\hline
\end{tabular}

\subsubsection{6 $<<$ Reaction $>>$}

\section{Definiton:}

See related concept.

\section{Related Concept:}

Reaction (3.2.3.9)

\section{Base Classes:}

\begin{tabular}{|l|l|}
\hline \multicolumn{1}{|c|}{ Base Class } & \multicolumn{1}{c|}{ Usage Rationale } \\
\hline Class & $\begin{array}{l}\text { To indicate that the class contains the logic (e.g. hardware or } \\
\text { executable software code) that constitutes and describes a reaction } \\
(3.2 .3 .9)\end{array}$ \\
\hline Operation & $\begin{array}{l}\text { To indicate that invoking the specified operation is a reaction } \\
(3.2 .3 .9) \text { to one or more events (3.2.3.8) of interest, and to further } \\
\text { describe the operation's effect on safety }\end{array}$ \\
\hline
\end{tabular}

Tags: (refer to section 3.2.3.9 for details)

\begin{tabular}{|l|l|l|l|}
\hline \multicolumn{1}{|c|}{ Name } & \multicolumn{1}{|c|}{ Type } & Multiplicity & \multicolumn{1}{c|}{ Description } \\
\hline Kind & Enumeration & {$[0 . .1]$} & See attribute: Kind \\
\hline
\end{tabular}




\begin{tabular}{|l|l|l|l|}
\hline \multicolumn{1}{|c|}{ Name } & \multicolumn{1}{|c|}{ Type } & Multiplicity & \multicolumn{1}{|c|}{ Description } \\
\hline ConsequenceOf & $\begin{array}{l}\text { Reference to a } \\
\text { model element } \\
\text { stereotyped } \\
<<\text { Event>> } \\
(5.2 .15) \text { (or its } \\
\text { subclass } \\
<<\text { Reaction>> } \\
(5.2 .16))\end{array}$ & $\begin{array}{l}\text { See } \\
\text { Consequence Of }\end{array}$ & \\
\hline When & Expression & {$\left[0 . .^{*}\right]$} & See attribute: When \\
\hline EffectOnSafetyDirection & Enumeration & {$\left[0 . .^{*}\right]$} & $\begin{array}{l}\text { See attribute: Effect On } \\
\text { Safety Direction }\end{array}$ \\
\hline EffectOnSafetyValue & Expression & {$\left[0 . .^{*}\right]$} & $\begin{array}{l}\text { See attribute: Effect On } \\
\text { Safety Value }\end{array}$ \\
\hline EffectOnSafetyContext & Expression & {$\left[0 .{ }^{*}\right]$} & $\begin{array}{l}\text { See attribute: } \\
\text { EffectOnSafetyContext }\end{array}$ \\
\hline
\end{tabular}

\subsubsection{7 <<SafetyCritical >}

\section{Definiton:}

See related concept.

\section{Related Concept:}

SafetyCritical (3.2.3.10)

Base Classes:

\begin{tabular}{|l|l|}
\hline \multicolumn{1}{|c|}{ Base Class } & \multicolumn{1}{c|}{ Usage Rationale } \\
\hline Class & $\begin{array}{l}\text { To indicate that the class is safety-critical (3.2.3.10) and specify } \\
\text { its safety (e.g. airworthiness) level }\end{array}$ \\
\hline Operation & $\begin{array}{l}\text { To indicate that invoking the operation is safety-critical (3.2.3.10) } \\
\text { and specify its safety (e.g. airworthiness) level }\end{array}$ \\
\hline
\end{tabular}




\begin{tabular}{|l|l|}
\hline \multicolumn{1}{|c|}{ Base Class } & \multicolumn{1}{c|}{ Usage Rationale } \\
\hline Relationship & $\begin{array}{l}\text { To indicate that the relationship is safety-critical (3.2.3.10) and } \\
\text { specify its safety (e.g. airworthiness) level }\end{array}$ \\
\hline
\end{tabular}

Tags: (refer to section 3.2.3.10 for details)

\begin{tabular}{|l|l|l|l|}
\hline \multicolumn{1}{|c|}{ Name } & \multicolumn{1}{|c|}{ Type } & Multiplicity & \multicolumn{1}{c|}{ Description } \\
\hline CriticalityLevel & Enumeration & {$[0 . .1]$} & See attribute: Criticality Level \\
\hline ConfidenceLevel & Enumeration & {$[0 . .1]$} & $\begin{array}{l}\text { See attribute: Confidence } \\
\text { Level }\end{array}$ \\
\hline TriggeredEvent & $\begin{array}{l}\text { Reference to a } \\
\text { model element } \\
\text { stereotyped } \\
<<\text { Event }>> \\
(5.2 .15) \text { (or its } \\
\text { subclass } \\
<<\text { Reaction>> } \\
(5.2 .16))\end{array}$ & \\
\hline
\end{tabular}

\subsubsection{8 <<Partition >>}

\section{Definiton:}

See related concept.

\section{Related Concept:}

Partition (3.2.3.11)

Base Classes:

\begin{tabular}{|l|l|}
\hline \multicolumn{1}{|c|}{ Base Class } & \multicolumn{1}{c|}{ Usage Rationale } \\
\hline Class & $\begin{array}{l}\text { To indicate that the class is has been partitioned (3.2.3.11) from } \\
\text { some other (usually more safety-critical (3.2.3.10)) class }\end{array}$ \\
\hline
\end{tabular}


Tags: (refer to section 3.2.3.11 for details)

\begin{tabular}{|l|l|l|l|}
\hline \multicolumn{1}{|c|}{ Name } & \multicolumn{1}{|c|}{ Type } & Multiplicity & \multicolumn{1}{c|}{ Description } \\
\hline PartitionedFrom & $\begin{array}{l}\text { Reference to a } \\
\text { class model } \\
\text { element } \\
\text { stereotyped } \\
<<\text { SafetyCritical }>>\end{array}$ & $\begin{array}{l}\text { See relationship: } \\
\text { Partitioned From } \\
(5.2 .17)\end{array}$ & \\
\hline Reference & $\begin{array}{l}\text { Reference to a } \\
\text { model element } \\
\text { stereotyped } \\
<<\text { Requirement }>> \\
(5.2 .9), \text { or a String }\end{array}$ & & See relationship: References \\
\hline Explanation & String & {$\left[0 . *^{*}\right]$} & See attribute: Explanation \\
\hline
\end{tabular}

\subsubsection{9 <<Handler $>>$}

\section{Definiton:}

See related concept.

\section{Related Concept:}

Handler (3.2.3.12)

Base Classes:

\begin{tabular}{|l|l|}
\hline \multicolumn{1}{|c|}{ Base Class } & \multicolumn{1}{c|}{ Usage Rationale } \\
\hline Class & To indicate that the class is an event (3.2.3.8) handler (3.2.3.12) \\
\hline
\end{tabular}


Tags: (refer to section 3.2.3.12 for details)

\begin{tabular}{|c|c|c|c|}
\hline Name & Type & Multiplicity & Description \\
\hline HandleableEvent & $\begin{array}{l}\text { Reference to a } \\
\text { model element } \\
\text { stereotyped } \\
<<\text { Event>> } \\
(5.2 .15) \text { (or its } \\
\text { subclass } \\
<<\text { Reaction〉> } \\
(5.2 .16))\end{array}$ & [1..*] & See relationship: Handles \\
\hline PerformedReaction & $\begin{array}{l}\text { Reference to a } \\
\text { model element } \\
\text { stereotyped } \\
\text { <<Reaction>> } \\
(5.2 .16)\end{array}$ & [1..*] & See relationship: Performs \\
\hline
\end{tabular}

\subsubsection{0 $<<$ Monitor $>>$}

\section{Definiton:}

See related concept.

\section{Related Concept:}

Monitor (3.2.3.13)

Base Classes:

\begin{tabular}{|l|l|}
\hline \multicolumn{1}{|c|}{ Base Class } & \multicolumn{1}{c|}{ Usage Rationale } \\
\hline Class & $\begin{array}{l}\text { To indicate that the class is a monitor (3.2.3.13) that monitors } \\
\text { some other class }\end{array}$ \\
\hline
\end{tabular}

Tags: (refer to section 3.2.3.13 for details)

\begin{tabular}{|l|l|l|l|}
\hline \multicolumn{1}{|c|}{ Name } & \multicolumn{1}{c|}{ Type } & Multiplicity & \multicolumn{1}{c|}{ Description } \\
\hline Kind & Enumeration & {$[0.1]$} & See attribute: Kind \\
\hline
\end{tabular}




\begin{tabular}{|c|c|c|c|}
\hline Name & Type & Multiplicity & Description \\
\hline MonitoredEntity & $\begin{array}{l}\text { Reference to a } \\
\text { class model } \\
\text { element } \\
\text { stereotyped } \\
<<\text { SafetyCritical } \gg> \\
(5.2 .17)\end{array}$ & {$\left[1 . *^{*}\right]$} & See relationship: Monitors \\
\hline DetectableEvent & $\begin{array}{l}\text { Reference to a } \\
\text { model element } \\
\text { stereotyped } \\
<<\text { Event }>> \\
(5.2 .15) \text { (or its } \\
\text { subclass } \\
<<\text { Reaction }>> \\
(5.2 .16))\end{array}$ & {$\left[1 . *^{*}\right]$} & See relationship: Detects \\
\hline EventHandler & $\begin{array}{l}\text { Reference to a } \\
\text { model element } \\
\text { stereotyped } \\
<<\text { Handler }>> \\
(5.2 .19)\end{array}$ & {$\left[0 . *^{*}\right]$} & See relationship: Notifies \\
\hline
\end{tabular}

\subsubsection{1 <<Simulator $>>$}

\section{Definiton:}

See related concept.

\section{Related Concept:}

Simulator (3.2.3.14) 
Base Classes:

\begin{tabular}{|l|l|}
\hline \multicolumn{1}{|c|}{ Base Class } & \multicolumn{1}{c|}{ Usage Rationale } \\
\hline Class & $\begin{array}{l}\text { To indicate that the class is a simulator (3.2.3.14) for some other } \\
\text { class or operation }\end{array}$ \\
\hline
\end{tabular}

Tags: (refer to section 3.2.3.14 for details)

\begin{tabular}{|l|l|l|l|}
\hline \multicolumn{1}{|c|}{ Name } & \multicolumn{1}{|c|}{ Type } & Multiplicity & \multicolumn{1}{c|}{ Description } \\
\hline SimulatedEntity & $\begin{array}{l}\text { Reference to a } \\
\text { class or operation } \\
\text { model element } \\
\text { stereotyped } \\
\text { <-SafetyCritical }>> \\
(5.2 .17)\end{array}$ & See relationship: Simulates & \\
\hline Parameter & Expression & {$\left[0 . *^{*}\right]$} & See attribute: Parameters \\
\hline
\end{tabular}

\subsubsection{2 $<<$ Strategy $>>$}

\section{Definiton:}

See related concept.

\section{Related Concept:}

Strategy (3.2.3.15)

\section{Base Classes:}

\begin{tabular}{|l|l|}
\hline \multicolumn{1}{|c|}{ Base Class } & \multicolumn{1}{c|}{ Usage Rationale } \\
\hline Collaboration & $\begin{array}{l}\text { To specify and describe a particular strategy (3.2.3.15) that is used } \\
\text { throughout the collaboration }\end{array}$ \\
\hline Class & $\begin{array}{l}\text { To specify and describe a particular strategy (3.2.3.15) that the } \\
\text { class implements }\end{array}$ \\
\hline
\end{tabular}


Tags: (refer to section 3.2.3.15 for details)

\begin{tabular}{|l|l|l|l|}
\hline \multicolumn{1}{|c|}{ Name } & \multicolumn{1}{|c|}{ Type } & Multiplicity & \multicolumn{1}{c|}{ Description } \\
\hline Kind & Enumeration & {$[0 . .1]$} & See attribute: Kind \\
\hline Parameter & Expression & {$\left[0 .{ }^{*}\right]$} & See attribute: Parameters \\
\hline DesignOf & $\begin{array}{l}\text { Reference to a } \\
\text { class or operation } \\
\text { model element } \\
\text { stereotyped } \\
<<\text { SafetyCritical }>>\end{array}$ & $\begin{array}{l}\text { See relationship: Describes } \\
\text { Design Of }\end{array}$ \\
\hline (5.2.17) & & \\
\hline
\end{tabular}

\subsubsection{3 $<<$ Formalism $>>$}

Definiton:

See related concept.

Related Concept:

Formalism (3.2.3.16)

Base Classes:

\begin{tabular}{|l|l|}
\hline \multicolumn{1}{|c|}{ Base Class } & \multicolumn{1}{c|}{ Usage Rationale } \\
\hline Collaboration & $\begin{array}{l}\text { To indicate that the collaboration is designed or verified by the } \\
\text { use of formal methods (3.2.3.16) }\end{array}$ \\
\hline Class & $\begin{array}{l}\text { To indicate that the class is designed or verified by the use of } \\
\text { formal methods (3.2.3.16) }\end{array}$ \\
\hline Operation & $\begin{array}{l}\text { To indicate that the operation is designed or verified by the use of } \\
\text { formal methods (3.2.3.16) }\end{array}$ \\
\hline
\end{tabular}

Tags: (refer to section 3.2.3.16 for details)

\begin{tabular}{|l|l|l|l|}
\hline \multicolumn{1}{|c|}{ Name } & \multicolumn{1}{|c|}{ Type } & Multiplicity & \multicolumn{1}{c|}{ Description } \\
\hline Method & Enumeration & {$\left[0 .{ }^{*}\right]$} & See attribute: Methods \\
\hline
\end{tabular}




\begin{tabular}{|l|l|l|l|}
\hline Name & \multicolumn{1}{|c|}{ Type } & Multiplicity & \multicolumn{1}{c|}{ Description } \\
\hline FormalismOf & $\begin{array}{l}\text { Reference to a } \\
\text { class or operation }\end{array}$ & & $\begin{array}{l}\text { See relationship: Describes } \\
\text { model element } \\
\text { stereotyped } \\
\end{array}$ \\
& Formalism Of \\
& $(5.2 .17)$ & & \\
\hline
\end{tabular}

\subsubsection{4 <CComplexity >}

\section{Definiton:}

See related concept.

\section{Related Concept:}

Complexity (3.2.3.17)

Base Classes:

\begin{tabular}{|l|l|}
\hline \multicolumn{1}{|c|}{ Base Class } & \multicolumn{1}{c|}{ Usage Rationale } \\
\hline Collaboration & $\begin{array}{l}\text { To describe a complexity (3.2.3.17) aspect (e.g. coupling using a } \\
\text { specific measure, .. etc) of a collaboration }\end{array}$ \\
\hline Class & $\begin{array}{l}\text { To describe a complexity (3.2.3.17) aspect (e.g. number of entry } \\
\text { points of code, ... etc) of a class }\end{array}$ \\
\hline Operation & $\begin{array}{l}\text { To describe a complexity (3.2.3.17) aspect (e.g. level of nesting, } \\
\ldots \text { etc) of an operation }\end{array}$ \\
\hline Relationship & $\begin{array}{l}\text { To describe a complexity (3.2.3.17) aspect (e.g. coupling using a } \\
\text { specific measure, ... etc) of a relationship }\end{array}$ \\
\hline
\end{tabular}

Tags: (refer to section 3.2.3.17 for details)

\begin{tabular}{|l|l|l|l|}
\hline \multicolumn{1}{|c|}{ Name } & \multicolumn{1}{|c|}{ Type } & Multiplicity & \multicolumn{1}{c|}{ Description } \\
\hline Measure & Enumeration & {$[0 . .1]$} & See attribute: Measure \\
\hline Value & Expression & {$[0 . .1]$} & See attribute: Value \\
\hline
\end{tabular}




\begin{tabular}{|c|c|c|c|}
\hline Name & Type & Multiplicity & Description \\
\hline ComplexityOf & $\begin{array}{l}\text { Reference to a } \\
\text { class or operation } \\
\text { model element } \\
\text { stereotyped } \\
<<\text { SafetyCritical»> } \\
(5.2 .17)\end{array}$ & {$[1 . . *]$} & $\begin{array}{l}\text { See relationship: Describes } \\
\text { Complexity Of }\end{array}$ \\
\hline
\end{tabular}

\subsubsection{5 <<Interface〉>}

\section{Definiton:}

See related concept.

\section{Related Concept:}

Interface (3.2.3.18)

Base Classes:

\begin{tabular}{|l|l|}
\hline \multicolumn{1}{|c|}{ Base Class } & \multicolumn{1}{c|}{ Usage Rationale } \\
\hline Class & $\begin{array}{l}\text { Indicates that the class acts as an interface (3.2.3.18) to some } \\
\text { other class }\end{array}$ \\
\hline
\end{tabular}

Tags: (refer to section 3.2.3.18 for details)

\begin{tabular}{|l|l|l|l|}
\hline \multicolumn{1}{|c|}{ Name } & \multicolumn{1}{|c|}{ Type } & Multiplicity & \multicolumn{1}{c|}{ Description } \\
\hline IsBetweenHardwareAndSoftware & Boolean & {$[0 . .1]$} & $\begin{array}{l}\text { See attribute: Is } \\
\text { Between } \\
\text { Hardware And } \\
\text { Software }\end{array}$ \\
\hline InterfaceFor & $\begin{array}{l}\text { Reference to a } \\
\text { class model } \\
\text { element } \\
\text { stereotyped } \\
\text { <SafetyCritical }>>\end{array}$ & $\begin{array}{l}\text { See relationship: } \\
\text { Is Interface For }\end{array}$ \\
& $(5.2 .17)$ & & \\
\hline
\end{tabular}




\begin{tabular}{|l|l|l|l|}
\hline \multicolumn{1}{|c|}{ Name } & \multicolumn{1}{|c|}{ Type } & Multiplicity & \multicolumn{1}{c|}{ Description } \\
\hline ProtocolID & String & {$[0 . .1]$} & $\begin{array}{l}\text { See attribute: } \\
\text { Protocol ID }\end{array}$ \\
\hline InputFunctionParameter & Expression & {$\left[0 . .^{*}\right]$} & $\begin{array}{l}\text { See attribute: } \\
\text { Input Function } \\
\text { Parameters }\end{array}$ \\
\hline OutputFunctionParameter & Expression & {$\left[0 . .^{*}\right]$} & $\begin{array}{l}\text { See attribute: } \\
\text { Output Function } \\
\text { Parameters }\end{array}$ \\
\hline
\end{tabular}

\subsubsection{6 $<<$ Concurrent $>$}

Definiton:

See related concept.

Related Concept:

Concurrent (3.2.3.19)

Base Classes:

\begin{tabular}{|l|l|}
\hline \multicolumn{1}{|c|}{ Base Class } & \multicolumn{1}{c|}{ Usage Rationale } \\
\hline Class & $\begin{array}{l}\text { To identify the concurrency (3.2.3.19) role (e.g. active, passive, } \\
\text { resource, } \ldots \text { etc) that a specific class assumes in the model }\end{array}$ \\
\hline
\end{tabular}

Tags: (refer to section 3.2.3.19 for details)

\begin{tabular}{|l|l|l|l|}
\hline \multicolumn{1}{|c|}{ Name } & \multicolumn{1}{|c|}{ Type } & Multiplicity & \multicolumn{1}{c|}{ Description } \\
\hline Role & Enumeration & {$[0 . .1]$} & See attribute: Role \\
\hline IsShared & Boolean & {$[0 . .1]$} & See attribute: Is Shared \\
\hline
\end{tabular}




\begin{tabular}{|l|l|l|l|}
\hline \multicolumn{1}{|c|}{ Name } & \multicolumn{1}{|c|}{ Type } & Multiplicity & \multicolumn{1}{c|}{ Description } \\
\hline TriggeredEvent & $\begin{array}{l}\text { Reference to a } \\
\text { model element } \\
\text { stereotyped }\end{array}$ & & See relationship: Triggers \\
& $<<$ Event>> & & \\
& $(5.2 .15)$ (or its & & \\
subclass & & \\
& $<<$ Reaction>> & & \\
& $(5.2 .16))$ & & \\
\hline Parameter & Expression & {$\left[0 .{ }^{*}\right]$} & See attribute: Parameters \\
\hline
\end{tabular}

\subsubsection{7 <<Defensive〉>}

Definiton:

See related concept.

\section{Related Concept:}

Defensive (3.2.3.20)

Base Classes:

\begin{tabular}{|l|l|}
\hline \multicolumn{1}{|c|}{ Base Class } & \multicolumn{1}{c|}{ Usage Rationale } \\
\hline Class & $\begin{array}{l}\text { To specify that the class employs a defensive programming } \\
\text { approach (3.2.3.20) and describes the reactions to actions that are } \\
\text { defended against }\end{array}$ \\
\hline Operation & $\begin{array}{l}\text { To specify that the operation employs a defensive programming } \\
\text { approach (3.2.3.20) and describes the reactions to actions that are } \\
\text { defended against }\end{array}$ \\
\hline
\end{tabular}

Tags: (refer to section 3.2.3.20 for details)

\begin{tabular}{|c|l|l|l|}
\hline \multicolumn{1}{|c|}{ Name } & \multicolumn{1}{|c|}{ Type } & Multiplicity & \multicolumn{1}{|c|}{ Description } \\
\hline DefendableInput & Expression & {$\left[1 .{ }^{*}\right]$} & $\begin{array}{l}\text { See attribute: Defendable } \\
\text { Inputs }\end{array}$ \\
\hline
\end{tabular}




\begin{tabular}{|l|l|l|l|}
\hline \multicolumn{1}{|c|}{ Name } & \multicolumn{1}{|c|}{ Type } & Multiplicity & \multicolumn{1}{c|}{ Description } \\
\hline Reaction & Reference to a & {$\left[1 . .^{*}\right]$} & See relationship: Performs \\
& model element & & \\
stereotyped & & \\
& <<Reaction>> & & \\
& $(5.2 .16)$ & & \\
\hline
\end{tabular}

\subsubsection{8 $<<$ Configurable $>>$}

\section{Definiton:}

See related concept.

\section{Related Concept:}

Configurable (3.2.3.22)

Base Classes:

\begin{tabular}{|l|l|}
\hline \multicolumn{1}{|c|}{ Base Class } & \multicolumn{1}{c|}{ Usage Rationale } \\
\hline Class & $\begin{array}{l}\text { To specify that the class can be configured (3.2.3.22) to produce a } \\
\text { different configuration (3.2.3.21) with a different behaviour }\end{array}$ \\
\hline
\end{tabular}

Tags: (refer to section 3.2.3.22 for details)

\begin{tabular}{|l|l|l|l|}
\hline \multicolumn{1}{|c|}{ Name } & \multicolumn{1}{|c|}{ Type } & Multiplicity & \multicolumn{1}{c|}{ Description } \\
\hline Kind & Enumeration & {$[0 . .1]$} & See attribute: Kind \\
\hline When & Enumeration & {$[0 . .1]$} & $\begin{array}{l}\text { See attribute: } \\
\text { When }\end{array}$ \\
\hline DefaultConfiguration & $\begin{array}{l}\text { Reference to a model } \\
\text { element stereotyped } \\
<<\text { ConfigurationContext>> } \\
(5.2 .8)\end{array}$ & $\begin{array}{l}\text { See relationship: } \\
\text { Is Defaulted To }\end{array}$ \\
\hline
\end{tabular}




\begin{tabular}{|c|c|c|c|}
\hline Name & Type & Multiplicity & Description \\
\hline ProducibleConfiguration & $\begin{array}{l}\text { Reference to a model } \\
\text { element stereotyped } \\
<<\text { ConfigurationContext }> \\
(5.2 .8)\end{array}$ & {$[1 . . *]$} & $\begin{array}{l}\text { See relationship: } \\
\text { Is Configurable To }\end{array}$ \\
\hline
\end{tabular}

\subsubsection{9 <<Loadable >}

\section{Definiton:}

See related concept.

\section{Related Concept:}

Loadable (3.2.3.23)

Base Classes:

\begin{tabular}{|l|l|}
\hline \multicolumn{1}{|c|}{ Base Class } & \multicolumn{1}{c|}{ Usage Rationale } \\
\hline Class & $\begin{array}{l}\text { To specify that the class can be loaded (3.2.3.23) on some other } \\
\text { configurable (3.2.3.22) class to produce a different configuration } \\
(3.2 .3 .21)\end{array}$ \\
\hline
\end{tabular}

Tags: (refer to section 3.2.3.23 for details)

\begin{tabular}{|l|l|l|l|}
\hline \multicolumn{1}{|c|}{ Name } & \multicolumn{1}{|c|}{ Type } & Multiplicity & \multicolumn{1}{|c|}{ Description } \\
\hline LoadableOn & $\begin{array}{l}\text { Reference to a model } \\
\text { element stereotyped } \\
<<\text { Configurable> }(5.2 .28)\end{array}$ & $\begin{array}{l}\text { See relationship: Is } \\
\text { Loadable On }\end{array}$ \\
\hline BaseConfiguration & $\begin{array}{l}\text { Reference to a model } \\
\text { element stereotyped } \\
<\text { ConfigurationContext>> } \\
(5.2 .8)\end{array}$ & $\begin{array}{l}{\left[0 . .^{*}\right]} \\
\text { Requires }\end{array}$ \\
\hline
\end{tabular}




\begin{tabular}{|l|l|l|l|}
\hline Name & \multicolumn{1}{|c|}{ Type } & Multiplicity & \multicolumn{1}{c|}{ Description } \\
\hline ResultantConfiguration & Reference to a model & {$\left[1 . .^{*}\right]$} & See relationship: \\
element stereotyped & & Produces \\
& $<<$ ConfigurationContext $>>$ \\
& $(5.2 .8)$ & & \\
\hline
\end{tabular}

\subsubsection{0 $<<$ Configurator $>$}

Definiton:

See related concept.

Related Concept:

Configurator (3.2.3.24)

Base Classes:

\begin{tabular}{|l|l|}
\hline \multicolumn{1}{|c|}{ Base Class } & \multicolumn{1}{|c|}{ Usage Rationale } \\
\hline Class & $\begin{array}{l}\text { To specify that the class can configure configurable (3.2.3.22) } \\
\text { classes to change the configuration (3.2.3.21) (and thus produce a } \\
\text { different behaviour) }\end{array}$ \\
\hline Operation & $\begin{array}{l}\text { To specify that the invoking the operation can configure } \\
\text { configurable (3.2.3.22) classes to change the configuration } \\
(3.2 .3 .21) \text { (and thus produce a different behaviour) }\end{array}$ \\
\hline
\end{tabular}

Tags: (refer to section 3.2.3.24 for details)

\begin{tabular}{|l|l|l|l|}
\hline \multicolumn{1}{|c|}{ Name } & \multicolumn{1}{|c|}{ Type } & Multiplicity & \multicolumn{1}{c|}{ Description } \\
\hline ConfigurableEntity & $\begin{array}{l}\text { Reference to a } \\
\text { model element } \\
\text { stereotyped }\end{array}$ & & See relationship: Configures \\
& $<<$ Configurable $>>$ & & \\
& $(5.2 .28)$ & & \\
\hline
\end{tabular}




\begin{tabular}{|l|l|l|l|}
\hline \multicolumn{1}{|c|}{ Name } & \multicolumn{1}{|c|}{ Type } & Multiplicity & \multicolumn{1}{c|}{ Description } \\
\hline ConfigurationEntity & $\begin{array}{l}\text { Reference to a } \\
\text { model element }\end{array}$ & & See relationship: Loads \\
& stereotyped & & \\
& <<Ladable > & & \\
& $(5.2 .29)$ & & \\
\hline
\end{tabular}

\subsubsection{1 <<Replicated $>>$}

Definiton:

See related concept.

\section{Related Concept:}

Replicated (3.2.3.25)

Base Classes:

\begin{tabular}{|l|l|}
\hline \multicolumn{1}{|c|}{ Base Class } & \multicolumn{1}{c|}{ Usage Rationale } \\
\hline Class & To indicate that class is replicated (3.2.3.25) \\
\hline
\end{tabular}

Tags: (refer to section 3.2.3.25 for details)

\begin{tabular}{|l|l|l|l|}
\hline \multicolumn{1}{|c|}{ Name } & \multicolumn{1}{|c|}{ Type } & Multiplicity & \multicolumn{1}{c|}{ Description } \\
\hline ID & String & {$[0 . .1]$} & See attribute: ID (Replicated) \\
\hline ReplicationGroupID & String & {$[0.1]$} & $\begin{array}{l}\text { Identifies the ReplicationGroup } \\
(3.2 .3 .27), \text { specified using the } \\
\text { <<ReliabilityContext > (5.2.2) } \\
\text { stereotype, which owns this } \\
\text { replicated instance. } \\
\text { See relationship: Owns } \\
\text { (ReplicationGroup) }\end{array}$ \\
\hline
\end{tabular}




\section{$5.2 .32<<$ Comparator $>$}

\section{Definiton:}

See related concept.

\section{Related Concept:}

Comparator (3.2.3.26)

Base Classes:

\begin{tabular}{|l|l|}
\hline \multicolumn{1}{|c|}{ Base Class } & \multicolumn{1}{c|}{ Usage Rationale } \\
\hline Class & $\begin{array}{l}\text { To indicate that the class is a comparator (3.2.3.26) that compares } \\
\text { the outputs of replicated (3.2.3.25) classes }\end{array}$ \\
\hline Operation & $\begin{array}{l}\text { To indicate that invoking the operation compares (3.2.3.26) the } \\
\text { outputs of replicated (3.2.3.25) classes }\end{array}$ \\
\hline
\end{tabular}

Tags: (refer to section 3.2.3.26 for details)

\begin{tabular}{|c|c|c|c|}
\hline Name & Type & Multiplicity & Description \\
\hline ReplicationGroupID & String & {$[0 . .1]$} & $\begin{array}{l}\text { Identifies the } \\
\text { ReplicationGroup (3.2.3.27), } \\
\text { specified using the } \\
\text { <<ReliabilityContext>> (5.2.2) } \\
\text { stereotype, which owns this } \\
\text { replicated instance. } \\
\text { See relationship: } \\
\text { (ReplicationGroup) }\end{array}$ \\
\hline PolicyParameter & $\begin{array}{l}\text { Expression or } \\
\text { Enumeration }\end{array}$ & {$\left[0 .{ }^{*}\right]$} & $\begin{array}{l}\text { See attribute: Policy } \\
\text { Parameters (Comparator) }\end{array}$ \\
\hline ComparedEntity & $\begin{array}{l}\text { Reference to a } \\
\text { model element } \\
\text { stereotyped } \\
<<\text { Replicated }>> \\
(5.2 .31)\end{array}$ & {$\left[2 . *^{*}\right]$} & $\begin{array}{l}\text { See relationship: Compares } \\
\text { (Comparator) }\end{array}$ \\
\hline
\end{tabular}




\subsection{Examples}

This section presents examples of software models using the proposed UML profile. The examples are explained in detail, and they serve to help the reader better understand the UML profile and how it can be used.This thesis discusses a total of nine examples (plus a case study in section 7). Three of those examples are discussed below as they primarily use concepts (and stereotypes) that are key to the airworthiness standard (e.g., the notion of software level) or that are not supported by other UML profiles. The reminaing six examples are discussed in . Appendix G. The examples are also stereotyped according to Gomaa's class classification as presented in [7] and summarized in Appendix E.

\subsubsection{Hardware/Software Interfaces}

The example in Figure 8 shows a Kalman filter and how it connects to the radar that provides its input and a simulator of the outside world's events.

Kalman filters are recursive functions. Therefore, Kalmanfilter is stereotyped with < ImplementationStyle〉> (5.2.11) whose "Kind" tagged value is set to "Recursive".

The project in which this system is developed has a coding standard requirement that says that "recursive algorithms shall not be used". Therefore the KalmanFilter class is also a deviation, or a violation, of the coding standard requirement, and is stereotyped with <Deviation> (5.2.10) whose "Kind" tagged value is set to "UsingRecursiveAlgorithm". The "Reference" tagged value is set to "CodingStandard" to indicate that using recursive algorithms is a deviation from, or a violation of, the project's coding standard. 
Furthermore, filters are time-related functions. Therefore, KalmanFilter is also stereotyped with $<<$ BehaviouralStyle > (5.2.12) whose "Kind" tagged value is set to "Time-Related".

Now, Kalman filters process radar outputs. Since this example has a software implementation for the Kalman filter, it has to interface with the actual radar hardware on the aircraft. For this reason, RadarInterface is available to provide an interface between the software Kalman filter and the hardware radar device. Thus, RadarInterface is stereotyped with $<$ Interface $>$ (5.2.25) whose "IsBetweenHardwareAndSoftware" tagged value is set to "true". The "InterfaceFor" tagged value indicates that RadarInterface is an interface for the actual radar hardware.

The testing of such systems is often performed in system integration labs. In other words, the software is not loaded on the aircraft and the aircraft flown just to perform software unit or integration testing. That would just be too expensive! Therefore, a software simulator is developed to simulate world events that happen outside of the aircraft. This simulator is RealWorldEventsimulator and is stereotyped with $<<$ Simulator $>$ (5.2.21). The "SimulatedEntity" tagged value indicates that it simulates the input to RadarInterface. Furthermore, the "SimulationParameter" tagged value indicates that the inputs exhibit a stochastic Poisson process with an average inter-arrival time of 20 milliseconds.

All the information described above is relevant for two purposes. First, it describes how software was designed to improve the development process - this was achieved, as described above, by using a simulator to improve the testing process and a 
hardware/software interface to provide communication between radar hardware and Kalman filter software. Simulators are often used to test systems [1]. Therefore, the whole diagram is stereotyped with $<<$ DesignContext $>>(5.2 .7)$ to indicate that this design setup is of special interest. Secondly, the modeled classes contain information that is relevant for the software certification aspects. For example, the airworthiness standard specifies that the certification authorities need to know about all hardware/software interfaces and deviations from plans or standards. Therefore, the diagram is stereotyped with $<<$ CertificationContext $>>(5.2 .6)$ to indicate that it contains information relevant for the certification of the software in this diagram.

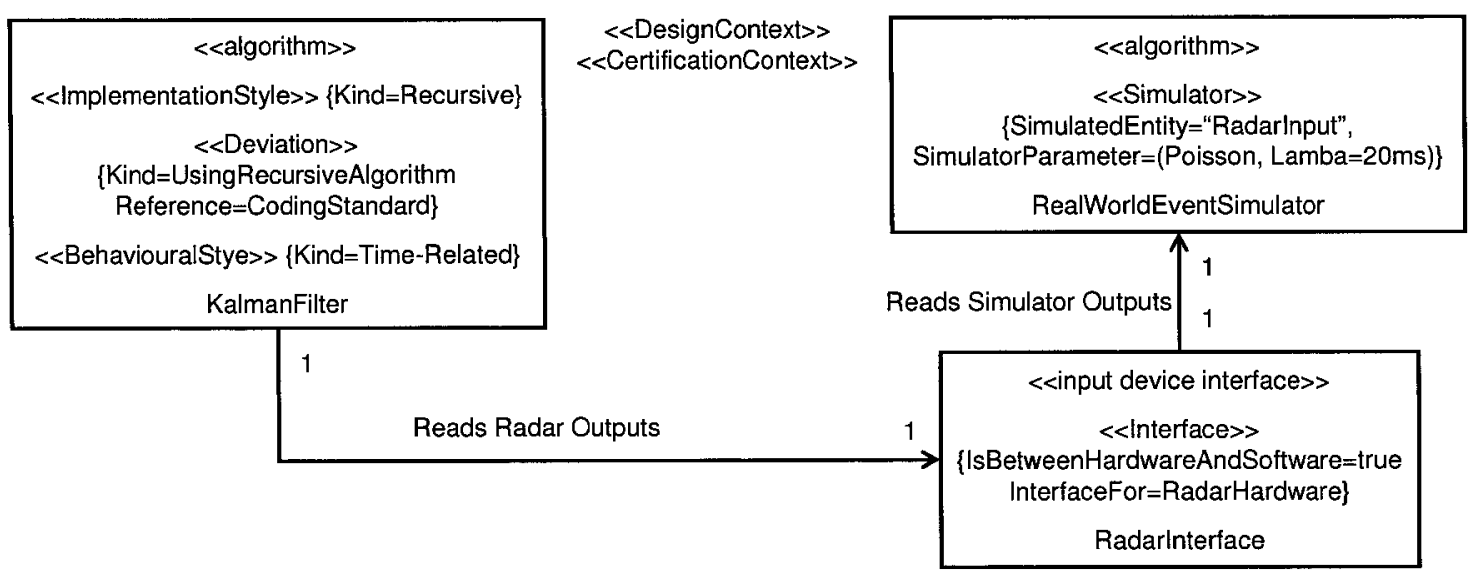

Figure 8: Kalman filter processing input, through an interface, from a simulator

(structure).

\subsubsection{Contributions to Failure Conditions}

The example Figure 9 shows software that controls the landing wheels of the aircraft.

PilotKeyboardInterface is an interface to the keyboard used by the aircraft's pilots to deploy or hide the landing wheels when desired. An aircraft normally has two pilots, so it 
is likely that there will be several keyboards that can command LandingWheelscontroller to deploy or hide the landing wheels.

Landingwheelscontroller communicates with the landing wheel hardware, through LandingWheelsInterface, and can command it to hide or deploy the wheels. The wheels are deployed when the aircraft is on the ground or about to land. Since LandingwheelsInterface is an interface for the landing wheel hardware, it is stereotyped with <<Interface>> (5.2.25), its "IsBetweenHardwareAndSoftware" tagged value is set to "true", and its "InterfaceFor" tagged value is set to "LandingWheels".

LandingWheelscontroller is a safety-critical element as well because it must ensure that the landing wheels are deployed when the aircraft is on the ground or it is at a low altitude because it is landing. If the landing wheels are not deployed when the aircraft is on the ground or is landing, then this could result in fatal injuries to the aircraft occupants. In the context of airworthiness, such software is assigned is assigned level B. Therefore, Landingwheelscontroller is stereotyped with $<<$ SafetyCritical $>$ (5.2.17) and its "CriticalityLevel" tagged value is set to "B". This is also why this diagram was stereotyped with $<<$ SafetyContext $>\quad(5.2 .1)$ Because it depends on Landingwheelsinterface, the airworthiness rules specify that LandingWheelsInterface must also be safety-critical and have a software level equal to at least the highest level of all classes that depend on it. In this example, Landingwheelsinterface is stereotyped with $\ll$ SafetyCritical $\gg$ (5.2.17) and is assigned "CriticalityLevel" equal to that of LandingWheelscontroller, namely level “B”. 
To ensure a higher level of safety, LandingWheelscontroller implements defensive programming mechanisms by ensuring that the pilot does not attempt to hide the landing wheels when they shouldn't. Defensive programming is common when developing user interfaces. In this example, Landingwheelscontroller defends against the pilot's attempt to hide the landing wheels when the aircraft's altitude is less than 100 meters by keeping the landing wheels deployed. This is explicitly specified by stereotyping Landingwheelscontroller with the <<Defensive>> (5.2.27) stereotype and specifying its "DefendableInput" and "Reaction" tagged values. (The KeepWheelsDeployed model element stereotyped $<<$ Reaction $>>$ is not shown in the diagram: It would be an operation of the LandingWheelsInterface class.)

Landingwheelscontroller determines the aircraft's altitude by reading the radar output from RadarInterface. RadarInterface was explained in detail in section 5.3.1. However, it has also been stereotyped here with $<<$ SafetyCritical $>$ (5.2.17) to explicitly specify the fact that some safety-critical functionality, such as Landingwheelscontroller, depends on it. Moreover, it has also been stereotyped with $<<$ Complexity $>>$ (5.2.24) and its "Measure" and "Value" tagged values indicate that the implementation must not have a "Big-O" larger than " $\mathrm{n}$ " (where the meaning of " $\mathrm{n}$ " is dependent on the algorithm in context). This is because radars usually have high frequency inputs, so the code of the corresponding interfaces must be optimized.

Furthermore, RadarInterface is monitored by RadarDatavalidator. RadarDatavalidator is a monitor whose purpose is to ensure that RadarInterface produces high-integrity information (i.e. high precision and accuracy). This is specified 
through the $<<$ Monitor $>$ (5.2.20) stereotype that is applied on RadarDatavalidator. Because the purpose of RadarValidatorDatavalidator is to ensure the integrity of the data, its "Kind" tagged value is set to "Integrity". Furthermore, the "MonitoredEntity" tagged value explicitly identifies the class that is monitored, namely RadarInterface. This is also why this diagram was stereotyped $<<$ IntegrityContext $>>(5.2 .3)$.

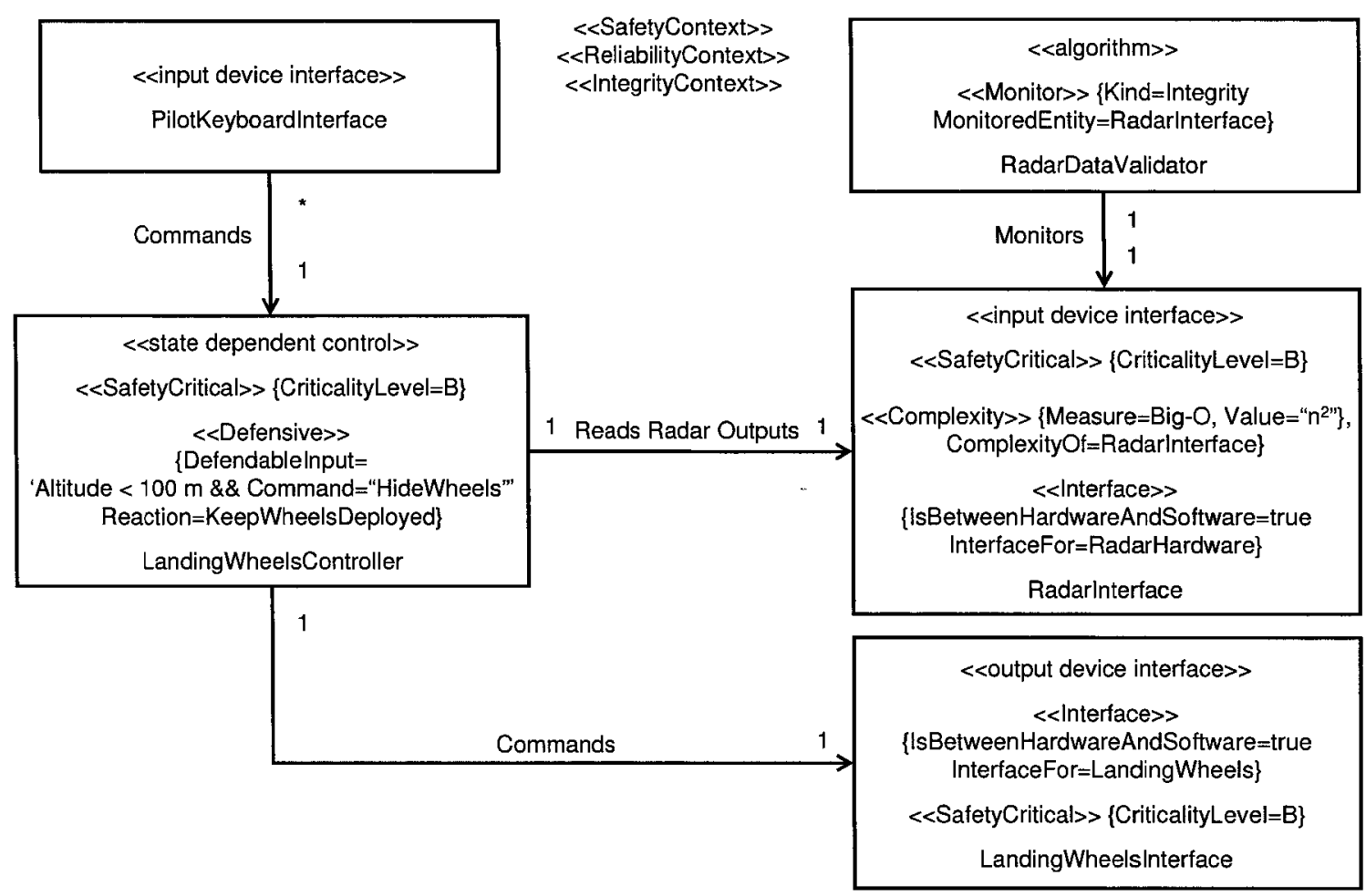

Figure 9: Landing wheel controller processing user and radar inputs (structure).

Finally, this diagram was also stereotyped with $<<$ ReliabilityContext $>>(5.2 .2)$ because it is crucial that the modeled classes be reliable to the requirements. For example, the requirements specified by the <<Defensive>> (5.2.27) stereotype on LandingWheelscontroller must be correctly implemented (e.g. it must not allow hiding the wheels for altitudes less than 100 meters, and not greater than 100 meters!). 


\subsubsection{Software Configurations}

The diagram in Figure 10 shows a configurable user interface. The user interface interacts with the users by displaying text in their language of preference. UserInterface itself is language-independent. It reads and displays textual strings in any of three languages: English, French, and German. This is achieved through UserInterfaceDictionary, which is stereotyped with $<<$ Configurable $>>(5.2 .28)$ to indicate that the user can change its configuration. The "Kind" tagged value is set to "Lookup-Table" and the "When" tagged value is set to "Run-Time" to indicate that look-up tables can be loaded into it at run-time to change its configuration. The "DefaultConfiguration" tagged value specifies that the "EnglishInterface" is the default configuration for UserInterfaceDictionary. The possible configurations that can result from such a load are listed in the "ProducibleConfiguration" tagged values. In this example, we have three possible configurations that can result from such a load: "EnglishInterface", "FrenchInterface", and "GermanInterface".

Dictionaryloader is the actual software than can perform the software load and therefore change the software configuration. Thus, it is stereotyped with $<<$ Configurator $>$ (5.2.29). The "ConfigurableEntity" tagged value identifies the class that can be configured, namely UserInterfaceDictionary. The "ConfigurationEntity" tagged values identify the classes that can be loaded on the "ConfigurableEntity", namely EnglishDictionaryTable, FrenchDictionaryTable, and GermanDictionaryTable, which are stereotyped with $<<$ Loadable $>(5.2 .29)$ to indicate that they can be loaded in appropriate situations. The "LoadableOn" tagged values are set to "UserInterfaceDictionary" to indicate that they are loadable on 
UserInterfaceDictionary, and the "ResultantConfiguration" specifies which configuration is produced by loading EnglishDictionaryTable, FrenchDictionarytable, and Germandictionarytable, which are EnglishInterface, FrenchInterface, and GermanInterface, respectively.

The diagram is stereotyped with $<<$ ConfigurationContext $>>(5.2 .8)$ to indicate that it contains information that is relevant to changing software configurations.

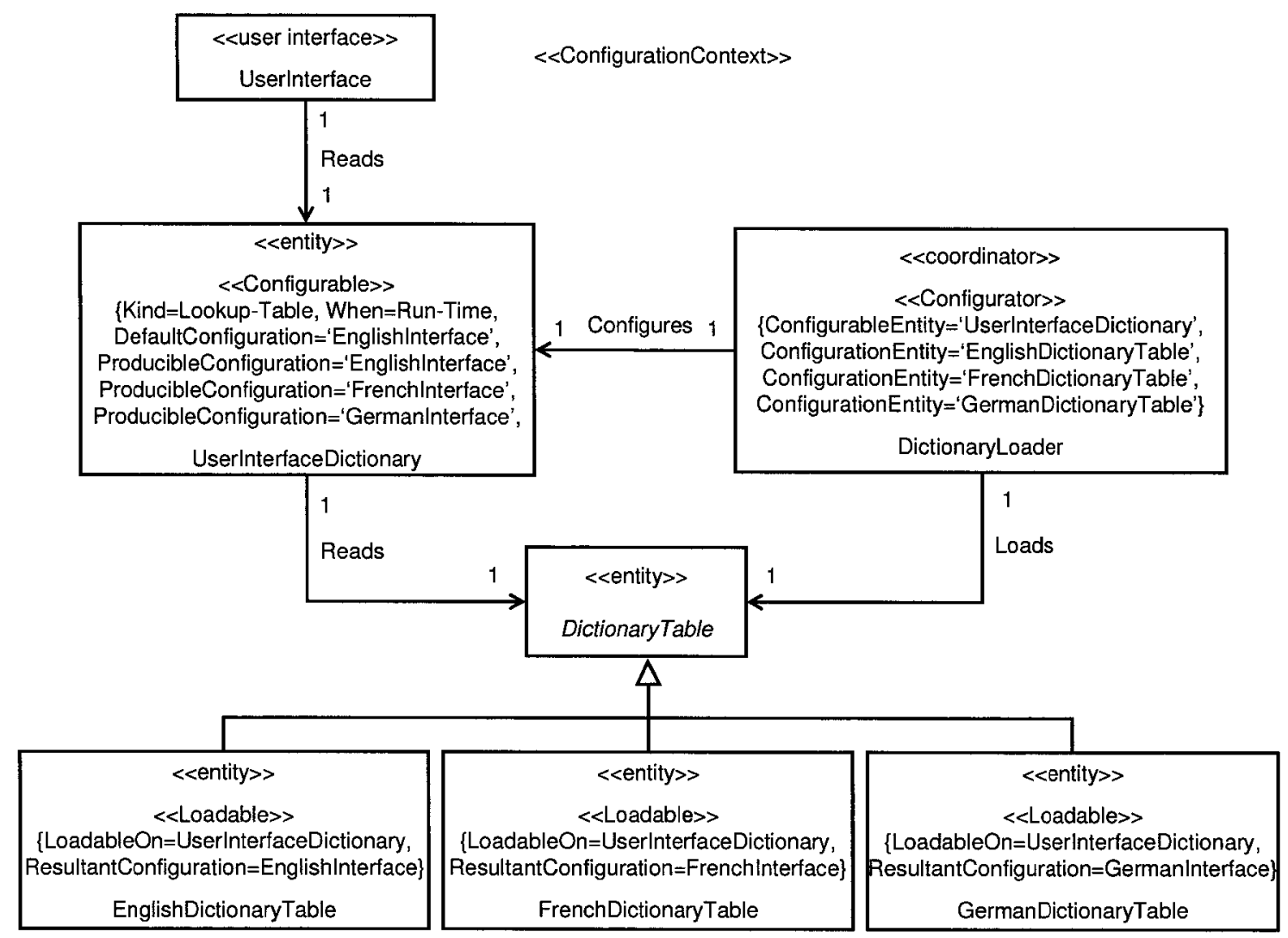

Figure 10: User interface language configurations (structure).

\subsection{Development Methodology}

The proposed UML profile provides a mechanism to model safety information in UML models. However, such mechanism is only a part of an overall process for developing 
airworthiness-compliant software. A methodology for developing airworthinesscompliant software products is shown in Figure 11. In practice, it is likely that a step starts before its previous step is fully completed and closed. The different steps are further described below.

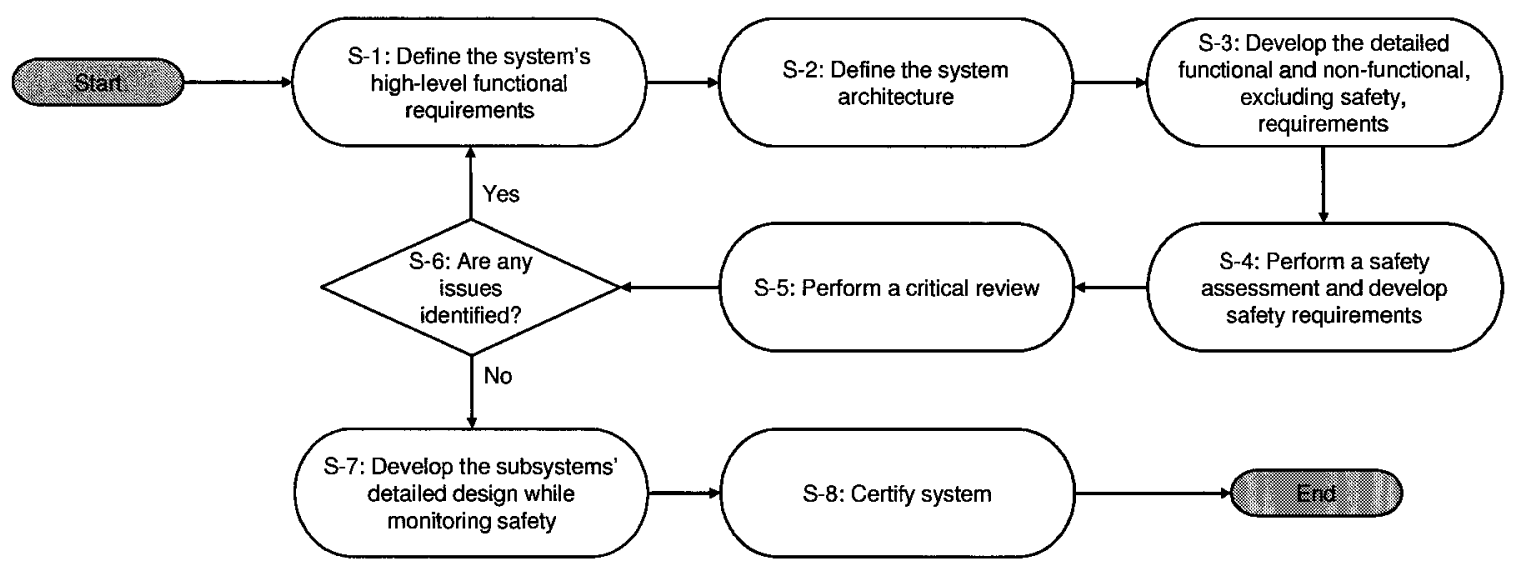

Figure 11: Development methodology of airworthiness-compliant software products.

Each step in Figure 11 is explained in Table 4.

\begin{tabular}{|c|l|}
\hline Step & \multicolumn{1}{|c|}{ Description } \\
\hline S-1 & $\begin{array}{l}\text { Define the system's high-level functional requirements } \\
\text { Activity: The system boundaries, behaviour, and high-level requirements are } \\
\text { defined. The system's boundaries define what consistutes part of the system, and } \\
\text { what does not. This is an important aspect for analysing the safety aspects of the } \\
\text { system, as whether a system is safe or not depends on what is considered part of } \\
\text { the system and what is not. In addition, the system's general behaviour, along with } \\
\text { the high-level functional requirements, are defined. At the end of this step, the } \\
\text { system's behaviour is understood and documented. } \\
\text { Output: The documented behaviour of the system, usually presented as the } \\
\text { system's high-level functional requirements based on the definition of the system } \\
\text { boundaries. }\end{array}$ \\
\hline
\end{tabular}




\begin{tabular}{|c|c|}
\hline Step & Description \\
\hline$S-2$ & $\begin{array}{l}\text { Define the system architecture } \\
\text { Activity: The system architecture is defined based on its high-level functional } \\
\text { requirements. The various subsystems are defined, and the role of each subsystem } \\
\text { in implementing the system's high-level functional requirements are defined and } \\
\text { documented. As a result, the high-level functional requirements for each subsystem } \\
\text { are defined. The proposed UML profile may be used to model the system } \\
\text { architecture. } \\
\text { Output: The system architecture, including the identification and definition of its } \\
\text { subsystems. }\end{array}$ \\
\hline$S-3$ & $\begin{array}{l}\text { Develop the detailed functional and non-functional, excluding safety, requirements } \\
\text { Activity: Detailed functional requirements are developed for the system and its } \\
\text { subsystems. In addition, non-functional requirements, excluding safety } \\
\text { requirements, are developed. } \\
\text { Output: Detailed functional and non-functional, excluding safety, requirements of } \\
\text { the system and its subsystems. }\end{array}$ \\
\hline S-4 & $\begin{array}{l}\text { Perform a safety assessment and develop safety requirements } \\
\text { Activity: By this stage, the behaviour of the system and its subsystems is already } \\
\text { understood. In this step, therefore, a safety assessment of the system is performed } \\
\text { based on its architecture and intended behaviour, and using one or more safety } \\
\text { assessment methods such as the ones identified in section } 2.1 \text {. The results of the } \\
\text { safety assessment are translated into safety requirements, and then the safety } \\
\text { requirements are assigned to the various subsystems. This step includes USAGE } 1 \\
\text { as defined in section } 2.4 \text {. } \\
\text { Output: Safety hazards identified in the safety assessment, and safety } \\
\text { requirements for the system and its subsystems. }\end{array}$ \\
\hline$S-5$ & $\begin{array}{l}\text { Perform a critical review } \\
\text { Activity: The output of steps } S-1-S-4 \text { are checked for consistency among each } \\
\text { other. Areas of interest in this step are whether the safety requirements are } \\
\text { complete with respect to the functional and non-functional requirements, and } \\
\text { whether there are any ambiguous, missing, or conflicting requirements. In addition, } \\
\text { the system architecture is analysed to determine whether there exists a more } \\
\text { suitable architecture for the identified safety requirements. Thus, the results of the } \\
\text { previous } S 1-S 4 \text { steps iteration are analysed, which will be later used to determine } \\
\text { whether another iteration is necessary or not. In practice, such critical reviews are } \\
\text { common to hold with the project's customer at selected milestones. } \\
\text { Output: A list of identified issues to be fixed. This list may be empty, but this will } \\
\text { be surprising unless steps S-1 - S-5 have already gone through at least two } \\
\text { iterations. }\end{array}$ \\
\hline
\end{tabular}




\begin{tabular}{|c|l|}
\hline Step & \multicolumn{1}{c|}{ Description } \\
\hline S-6 & $\begin{array}{l}\text { Are any issues identified? } \\
\text { Activity: The results from step S-5 are analysed. If any issues are encountered, } \\
\text { step S-1 is revisited to correct all identified issues. Otherwise, development is } \\
\text { progressed to step S-7. } \\
\text { Output: The decision on whether to perform another iteration of steps S-1 - S-5 or } \\
\text { not. }\end{array}$ \\
\hline S-7 & $\begin{array}{l}\text { Develop the subsystems' detailed design while monitoring safety } \\
\text { Activity: The subsystems' detailed design is developed. The detailed design will } \\
\text { also include the specification of the system's events of interest, and the desired } \\
\text { reactions to those events. The definition of the events and reactions will depend on } \\
\text { the safety requirements of the system. The proposed UML profile is used to design } \\
\text { the subsystems (USAGE 2 in section 2.4), and the design decisions are justified } \\
\text { (USAGE 3 in section 2.4). The design elements are traced back to the requirements } \\
\text { using the proposed UML profile's stereotypes. While the subsystems are being } \\
\text { designed, the design's conformance to the safety requirements is continuously } \\
\text { monitored (USAGE 4 in section 2.4). In practice, software implementation also } \\
\text { occurs in this step. } \\
\text { Output: The detailed design of the subsystems, the system events and reactions, } \\
\text { the justifications of the design decisions, and the safety monitoring information. }\end{array}$ \\
\hline S-8 & $\begin{array}{l}\text { Certify system } \\
\text { Activity: The project's safety and airworthiness engineers are engaged with the } \\
\text { certification authority to demonstrate the project's compliance with the } \\
\text { certification requirements. In this step, safety information is obtained from the } \\
\text { software (USAGE 5 in section 2.4), and evidence of performing relevant activities } \\
\text { (such as USAGE 1, USAGE 2, USAGE 3, and USAGE 4 in section 2.4) are } \\
\text { presented. Any issues identified by the certification authority are corrected and the } \\
\text { certification is ensured to completion. } \\
\text { Output: Successful certification of the system. }\end{array}$ \\
\hline
\end{tabular}

Table 4: Details of the development methodology steps. 


\section{GENERATION OF CERTIFICATION INFORMATION}

Now that a UML profile is defined, it can be used to model software that requires certification according to the airworthiness standard. This section shows how a UML model using the proposed UML profile can be used to automatically generate information that can either be submitted to the certification authorities, or can be used to track issues of relevance to the certification authorities. In either case, this information improves communication between airworthiness engineers and software engineers. The information required for submission to the certification authorities for each software level is listed in section 11 and annex A in the airworthiness standard, RTCA DO-178B [4].

\subsection{Technological Requirements}

In order to be able to generate certification information from UML models, there are technological requirements that software development tools must support. Those requirements can be summarized in one requirement, namely the ability to search UML models based on the stereotypes that are applied to model elements and the values of the stereotypes' tagged values.

For example, consider a scenario when one needs to identify all safety-critical model elements. In this case, the modeling tool must be able to search the UML model and identify all model elements, such as classes in class diagrams, that have been stereotyped with $<<$ SafetyCritical $\gg>$ (5.2.17). If the user of the tool needs to identify all safetycritical model elements that are assigned software level $\mathrm{A}$, for example, then the tool must be able to read the "CriticalityLevel" tagged value of the $<<$ SafetyCritical $>$ 
(5.2.17) stereotype and identify when it is set to "A". This is why the proposed UML profile emphasizes specifying information in machine-readable language.

In practice, there are several possible methods to achieve this technological requirement of being able to search UML models. Below is a list of some methods to guide the users of the proposed UML profile.

\subsubsection{Integrated Support in UML Modeling Tools}

One convenient method to extract safety information from UML models is to provide a mechanism to do so in the UML modeling tool itself. UML modeling tools already offer some sort of search capabilities for the designers. For example, ARTiSAN Studio [31], which is a UML modeling tool from ARTiSAN Software Inc., allows designers to identify where specific model elements are used. That is one example of ARTiSAN's search capabilities. Another example of a modeling tool is International Business Machines Corporation's (IBM) Rational Software Architect [32]. A third example is Telelogic's Rhapsody [33]. This UML model-driven development tool allows designers to search UML models for uses of specific stereotypes. In addition, it supports the Visual Basic for Applications (VBA) scripting language, which allows modelers to write their own scripts and execute them on models. This could be used to perform search queries.

The Eclipse Modeling Framework (EMF) for Java [34] is a popular, and easily extensible, software development framework. Some of EMF's extensions include the capability to use the Object Constraint Language (OCL) [35] to specify search queries on UML models, and then write Java code to execute them. Examples of the search capabilities of this technology include the capability to search for all objects that are 
instances of a specific class. However, the current state of this technology does not support searching UML models based on the stereotypes applied to model elements and the values assigned to the tagged values. Nevertheless, the integration of EMF and OCL is a promising approach that should be easibly extensible to support search queries based on stereotypes and tagged values.

\subsubsection{Exporting UML Models using XMI}

XMI [36] is an OMG standard for representing, and therefore exchanging, models and metadata in an XML-based language.

In practice, UML modeling tools can export UML models in the XMI language. This would create an XML file containing all the model data. Since XMI is a standard format, it can be imported by any other tool, thus establishing a common format across different tools. For example, imagine project A that is using Rational Software Architect to model its software. Further, assume that project $\mathrm{A}$ is similar in nature to a previously completed project B that used ARTiSAN Studio to model its software. Project A now wants to reuse the software model from project B. To achieve this, one would use a feature from ARTiSAN Studio that would export an XMI file containing all the software model for

project B. Then, project A can import this XMI file using an import XMI file feature in Rational Software Architect. Thus, the model would be transfered from project B to project A.

In our case of generating certification information, the model would be exported from the modeling tool by generating an XMI file. Once an XMI file containing the software model is available, it can be easily parsed and searched. This is possible in many ways, 
but the most appropriate method would probably involve the use of the Extensible Stylesheet Language (XSL) [37]. XSL is used to parse and transform XML-based files, such as XMI files, to any desired format. Such transformation is achieved by using XSL files called stylesheets. The purpose of XSL stylesheets is then to display the same model data in different formats or views, just like the Model-View-Controller (MVC) software pattern displays the same model information in different views. Using this approach, XSL stylesheets can be developed to execute search queries on the model data in the XMI files.

\subsection{Examples}

The examples presented below are specified in a high-level Structured Query Language (SQL)-like language. It is intended to be pseudocode-like, result in shorter text (see below), and be implementation-independent. Developers can also use them to implement using existing development tools and frameworks (refer to documentation of the existing tools and frameworks for more detail). It does not require the reader to have any knowledge of other tools or frameworks. The grammar for the language used is specified in an extension of the Extended Backus-Naur Form (EBNF), which is a popular syntax for specifiying languages [38]. The EBNF language itself is specified in the EBNF standard [39]. One popular example of a language specified in EBNF is the Ada language as specified in the Ada 95 Language Reference Manual [40].

The EBNF meta-symbols used in this section are:

1. “::=" means "is defined as". 
2. "p" means "or".

3. Literal strings are enclosed in double-quotes “"”.

4. Angle brackets "<>" are used around category names.

5. Optional items (zero or one) are enclosed in square brackets "[]".

6. Repetitive items (zero or more) are enclosed in braces " \{\} ".

The grammar for the search language used in this section, represented in EBNF form, is:

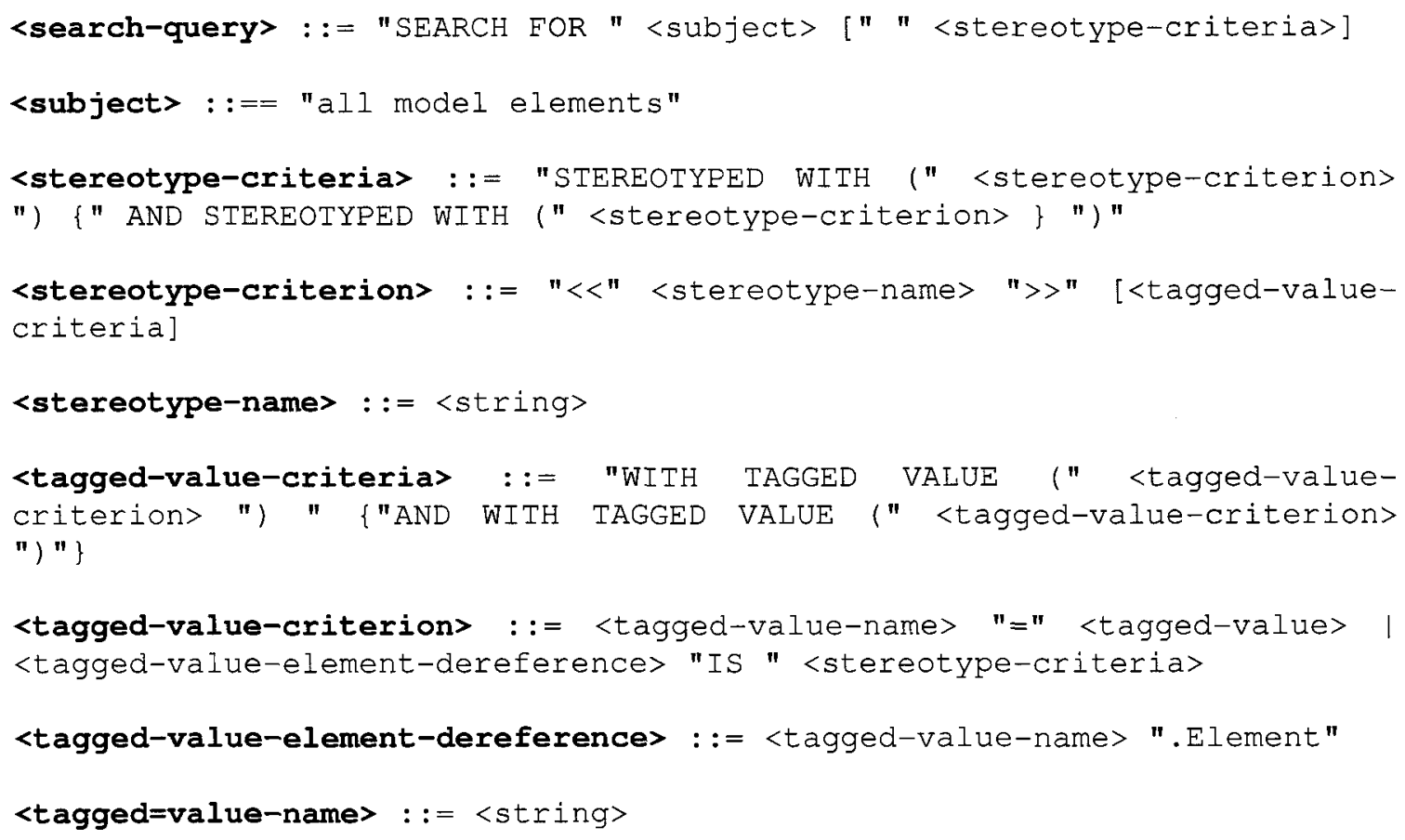

The <tagged-value-element-dereference> is used to obtain the model element to which a tagged value refers.

\subsubsection{Hardware/Software Interfaces}

Section 11.1 bullet a. in the airworthiness standard RTCA DO-178B [4] requires that the project's Plan for Software Aspects of Certification (PSAC), which is submitted to the 
certification authority, include a description of hardware/software interfaces in the system. Furthermore, section 11.9 bullet $\mathrm{f}$. in the airworthiness standard RTCA DO-178B [4] requires that hardware/software interfaces be documented and the requirements of their protocols, frequency of input, and frequency of outputs be presented.

A list of hardware/software interfaces can be extracted from the model using the following search query:

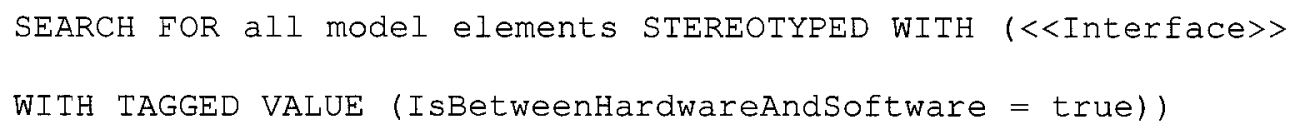

From the results of this query, the "ProtocolID", "InputFunctionParameter", and “OutputFunctionParameter" tagged values of the $<<$ Interface $>>(5.2 .25)$ stereotype can be read to present the information described above. For example, executing this search query on the model in Figure 8 gives the following results: Radar Interface.

\subsubsection{Contributions to Failure Conditions}

Section 11.1 bullet $\mathrm{c}$. in the airworthiness standard RTCA DO-178B [4] requires that the project's PSAC include a description of software's contributions to failure conditions.

A list of software that can contribute to failure conditions, along with the severity of the failure conditions, can be extracted from the model using the following search query:

SEARCH FOR all model elements STEREOTYPED WITH
(<<SafetyCritical >)

From the results of this query, the "CriticalityLevel" tagged value of the $<<$ SafetyCritical $>>(5.2 .17)$ stereotype can be read to identify the failure condition levels 
that each safety-critical software component contributes to. For example, executing this search query on the model in Figure 9 gives the following results: LandingWheels Controller, LandingWheelsInterface, RadarInterface.

\subsubsection{COTS Software}

Section 11.1 bullet $\mathrm{g}$. in the airworthiness standard RTCA DO-178B [4] requires that the project's PSAC include a description of COTS software used.

A list of COTS software can be extracted from the model using the following search query:

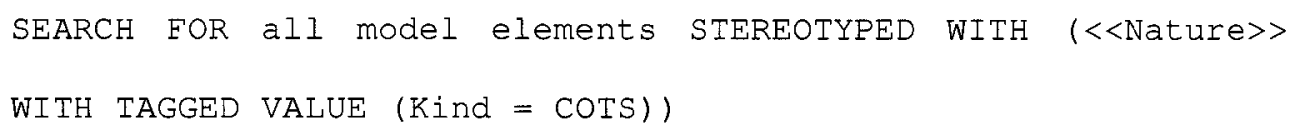

For example, executing this search query on the model in Figure 17 (discussed in Appendix G.1) gives the following results: SafeFlightPaths.

\subsubsection{Software Partitioning}

Section 11.3 bullet $f$. in the airworthiness standard RTCA DO-178B [4] requires that the project specify which methods are used to verify the integrity of partitions performed. Furthermore, section 11.9 bullet $\mathrm{h}$. requires that partitioning requirements allocated to software, as well as the software level(s) for each partition, be specified.

A list of partitions can be extracted from the model using the following search query:

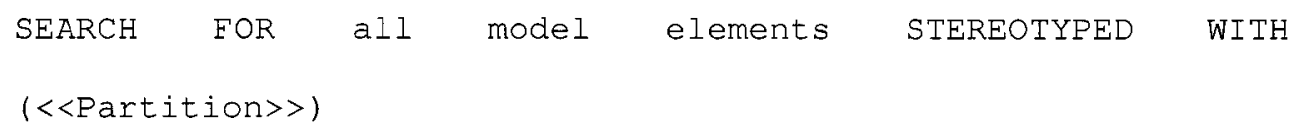


From the results of this query, the "PartitionedFrom" tagged value of the $<<$ Partition $>>$ (5.2.18) stereotype can be read to determine which software component this partition was partitioned from. Furthermore, the "Reference" tagged value of the $<$ Partition $>>$ (5.2.18) stereotype can be read to determine the requirement that resulted in this partition. For example, executing this search query on the model in Figure 18 (discussed in Appendix G.2) gives the following results: AutoPilotController, ConvertiblesteeringInformation.

A list of partitions that have been assigned software levels, along with the software level for each partition, can be extracted from the model using the following search query:

SEARCH FOR all model elements STEREOTYPED WITH
$(<<$ Partition>>) AND STEREOTYPED WITH (<<SafetyCritical>>)

From the results of this query, the "CriticalityLevel" tagged value of the $<<$ SafetyCritical $>(5.2 .17)$ stereotype can be read to determine the software level for each partition. For example, executing this search query on the model in Figure 18 gives the following results: AutoPilotController.

\subsubsection{Requirements and Traceability}

Section 11.9 in the airworthiness standard RTCA DO-178B [4] requires that the software requirements data be available. Furthermore, the airworthiness standard RTCA DO-178B [4] requires that the software design (e.g. UML model) be traced to the software requirements for software assigned level D or above. It also requires that the source code be traceable to the requirements for software assigned level $\mathrm{C}$ or above. 
A list of model elements traceable to software requirements can be extracted from the model using the following search query:

SEARCH FOR all model elements STEREOTYPED WITH
$(<<$ Requirement $>>)$

For example, executing this search query on the model in Figure 19 (discussed in Appendix G.3) gives the following results: PilotKeyboardinterface, Commands, and the diagram itself. Those are stereotype with $<<$ Requirement>> (5.2.9) whose IDs, respectively, are: IREQ 1, LREQ 2, HREQ 1.

For requirements that are only relevant for safety purposes, the following search query can be used:

SEARCH FOR all model elements STEREOTYPED WITH
$(<<$ Requirement>> WITH TAGGED VALUE (Kind $=$ Safety $))$

For example, executing this search query on the model in Figure 19 (discussed in appendix G.3) gives the following results: PilotKeyboardinterface, Commands, and the diagram itself. Those are stereotype with $<<$ Requirement»> (5.2.9) whose IDs, respectively, are: LREQ 1, LREQ 2, HREQ 1.

For a list of all design decisions that are a result of safety-related requirements, the following search query can be used:

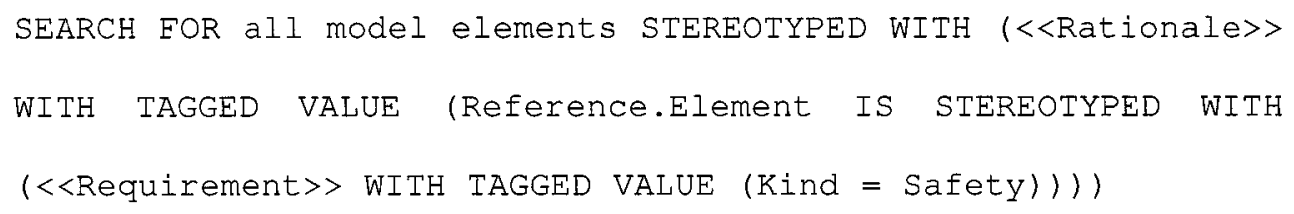


The search query can be read as follows: Find all model elements that are stereotyped with $<<$ Rationale $>$ (5.2.14). Then, pick only those elements where the "Reference" tagged value points to safety requirements (as indicated in the model with the $<<$ Requirement > (5.2.9) stereotype and its "Kind" tagged value). Thus, the above search query specifies that the results are all model elements that are stereotyped with $<<$ Rationale > (5.2.14), and where the "Reference" tagged value of the $<<$ Rationale > (5.2.14) stereotype is a reference to another model element stereotyped with $<<$ Requirement $>$ (5.2.9) whose "Kind" tagged value is "Safety". For example, executing this search query on the model in Figure 19 gives the following result: SafeFlightPaths.

\subsubsection{Multiple-Version Dissimilar Software}

Section 11.1 bullet $\mathrm{g}$. in the airworthiness standard RTCA DO-178B [4] requires that the project's PSAC include a description of the multiple-version dissimilar software used. Furthermore, section 11.3 bullet $\mathrm{j}$. in the airworthiness standard RTCA DO-178B [4] requires that a description of the software verification process activities used to verify multiple-version dissimilar software be presented.

A list of multiple-version dissimilar software can be extracted from the model using the following search query:

SEARCH FOR all model elements STEREOTYPED WITH
$(<<$ Replicated $>>)$


For example, executing this search query on the model in Figure 20 (discussed in Appendix G.4) gives the following results: Radarfilter1, Radarfilter2, RadarFilter3.

\subsubsection{Recursive Software}

Section 11.7 bullet e. in the airworthiness standard RTCA DO-178B [4] requires that the software design standards specify which constraints on the software design exist. Such design constraints could require the exclusion of software recursion.

If software recursion is used, then its use can be identified in the model. A list of recursive software can then be extracted from the model using the following search query:

SEARCH FOR all model elements STEREOTYPED WITH
$(<<$ Implementationstyle>> WITH TAGGED VALUE (Kind =
Recursive))

For example, executing this search query on the model in Figure 8 gives the following results: KalmanFilter.

If recursive software is not permitted by the software design standard but this rule was broken for some reason in some place, then a list of similar deviations from the standard can be extracted using the following search query:

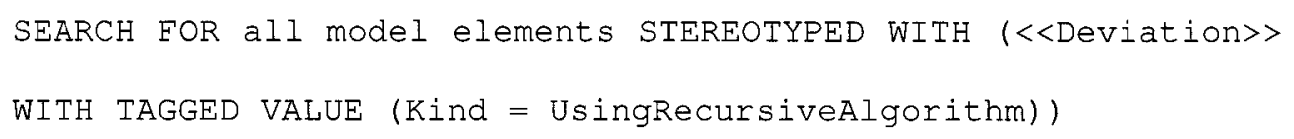


From the results of this query, the "Reference" and "Explanation" tagged values of the $<<$ Deviation $>>(5.2 .10)$ stereotype can be read to determine the standard from which this deviation existed and the rationale for this deviation. For example, executing this search query on the model in Figure 8 gives the following results: KalmanFilter 


\section{CASE STUDY - NAVIGATION CONTROLLER}

In this section, an aircraft's navigation system is analysed, and its navigation controller subsystem is designed using the proposed UML profile (section 5.1). The goal is to demonstrate, through a realistic case study the usefulness of the profile in the context of the usage scenarios we defined (section 2.4). In order to be complete and provide enough insights about the system to the reader, we also go through the development methodology described in section 5.4.

Here is a mapping between the steps of the development methodology (Figure 11 in section 5.4) and the subsections where they are addressed:

S-1 Define the system's high-level functional requirements: In section 7.1, an overview of the system is introduced, which describes in high-level language the major functionalities of the system.

S-2 Define the system architecture: The system architecture is presented and explained in section 7.2 .

S-3 Develop the detailed functional and non-functional requirements (excluding safety): The functional requirements of the subsystem under study are presented and explained in section 7.3 .

S-4 Perform a safety assessment and develop safety requirements: A safety assessment is performed in section 7.4 using four standard, complementary 
methods. Its results are presented in sections 7.4.1 - 7.4.4, from which the safety requirements are derived and presented in section 7.4.5.

S-5 Perform a critical review: Due to space constraints, only the final results are presented in this case study rather than the actual iterations used to develop the system. Therefore, the results of this step are not presented.

S-6 Are any issues identified?: For the same reasons as above, the results of this step are not presented.

S-7 Develop the subsystems' detailed design while monitoring safety: The design of the subsystem under study, including aspects related the defined safety requirements, is presented in section 7.5.

S-8 Certify system: In section 7.6, the usage scenarios of our proposed UML profile are discussed in the context of our case study. Their results are generally submitted to the certification authorities to certify the system, particularly the results of usage USAGE 5 (Get Safety Information), which are discussed in section 7.6.5.

\subsection{Overview}

The navigation controller subsystem is used to control the aircraft's flight paths through both automatic pilot and manual input from the pilots. 
It is worth reviewing the following terminology before proceeding with the case study:

1. Fly-To-Point (FTP): An FTP specifies a location on earth that the aircraft plans to fly to. For example, an aircraft flying from London to Paris will have at least one FTP, which is Paris.

2. Latitude/Longitude (LAT/LONG): A LAT/LONG specifies a particular geographic position on earth in latitude and longitude. LAT/LONG values are the standard measures for specifying geographic positions in navigation systems. The unit of both LAT and LONG are degrees, with LAT ranging from 90 South to 90 North, and Longitude ranging from 180 Westward to 180 Eastward. LAT/LONG values are generally used to specify FTP positions.

3. Bearing: The bearing on an aircraft is the direction in which it is flying. The bearing is generally specified in degrees, with a range of $[0,360[$. A bearing of zero is in a direction starting from the aircraft's position and towards the North Pole.

4. Dead-Reckoning: Dead-reckoning an aircraft's position means that the aircraft's position is being approximated using a previously known position at a given point of time, the current speed and bearing (direction). Ideally, the navigation system uses a Global Positioning System (GPS) to determine the aircraft's LAT/LONG position. If the GPS system fails, however, the navigation system can then approximate the position by dead-reckoning it. 
The aircraft's navigation controller subsystem has the following primary responsibilities:

1. Autopilot (Automatic): Based on the source and destination of the aircraft, this subsystem can choose an appropriate flight path. During the entire flight period, it can also guide the aircraft by generating appropriate commands to the aircraft's wings and engines to change the speed and bearing (i.e. direction) as required.

2. Supporting Custom Flight Paths (Semi-Automatic): This subsystem can accept commands from the pilots such as a specific position's latitude and longitude (LAT/LONG). Then, it controls the aircraft's speed and bearing to get to the desired FTP that was indicated by the pilot.

In order to perform such functionality, this subsystem needs to have continuous input from the aircraft's navigation system, which reports the current position and altitude of the aircraft at all time. In addition, it needs to be able to command the aircraft's wings and engines to change the speed and bearing.

\subsection{System Architecture}

Recall that software safety is only meaningful within the context of the system in which the software is used. As a result, it is mandatory to consider the system architecture as a whole to determine the safety aspects of NavigationControllerSubsystem. The system architecture, in which Navigationcontrollersubsystem appears, is shown in Figure 12 , and is discussed below. In the discussion, the rationale for assigning each software level is explained. The software levels were defined in section 2.2. 


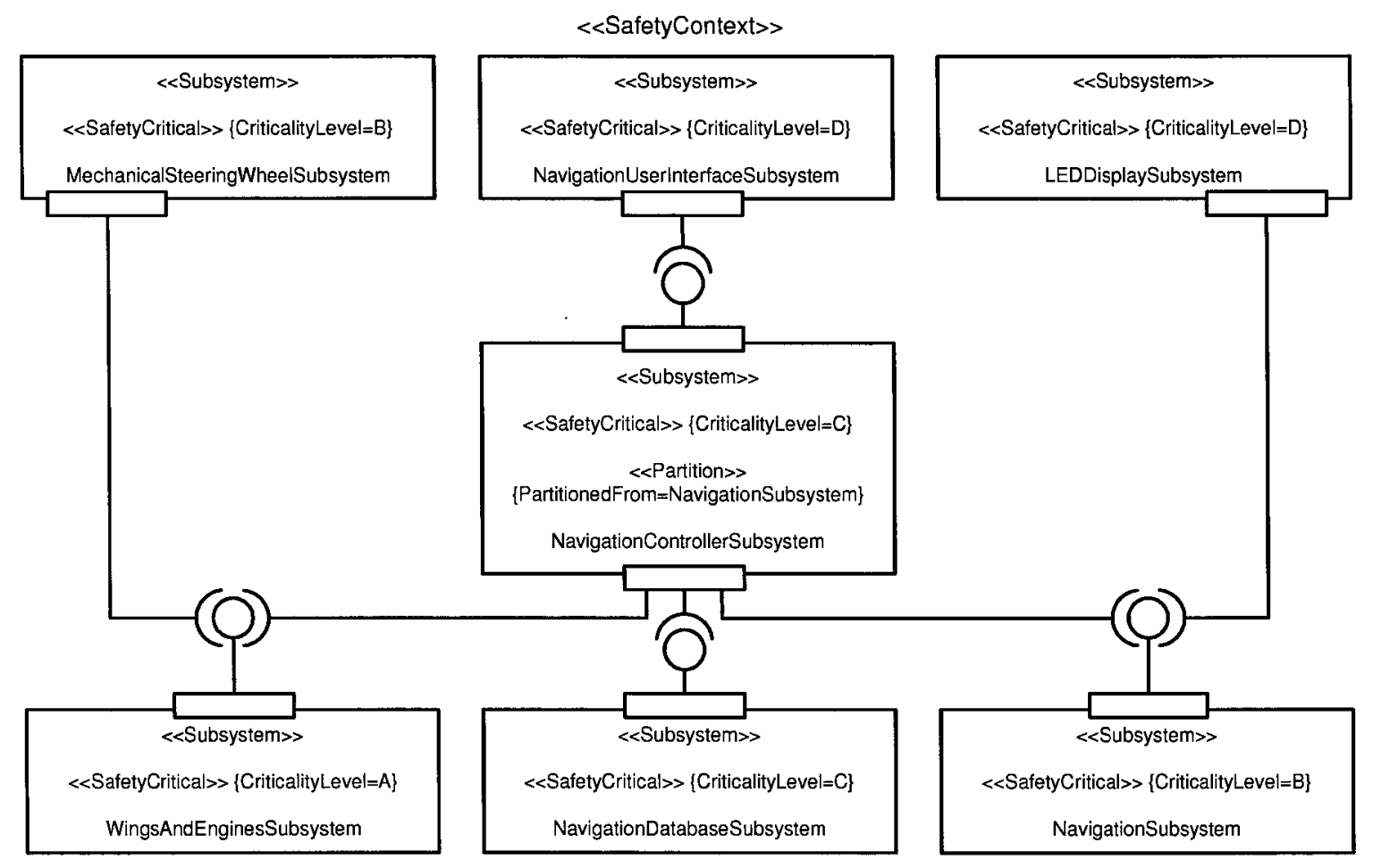

Figure 12: System architecture (structure).

The system is composed of the following subsystems:

1. WingsAndEnginesSubsystem: This subsystem represents the wings and the engines of the aircraft, and is used to control them. Therefore, it is the most important element to control the aircraft's speed and bearing. Thus, it is safetycritical and is assigned level A, as the $\ll$ SafetyCritical» (5.2.17) stereotype illustrates, because its failure prevents the continued safe flight and landing. This subsystem will not be considered any further in this case study.

2. MechanicalSteeringWheelsubsystem: This subsystem represents the pilots' mechanical steering wheel. They can use it to manually change the aircraft's speed and bearing. Thus, it is safety-critical and is assigned level B, as the $<<$ SafetyCritical $>$ (5.2.17) stereotype illustrates, because its failure prevents the 
pilots from performing their tasks correctly and accurately, but the aircraft's speed and bearing can still be controlled by other subsystems. This subsystem will not be considered any further in this case study.

3. NavigationSubsystem: This subsystem represents the navigation system that determines the current position, altitude, speed, and bearing of the aircraft through a GPS system and other technologies. Thus, it is safety-critical and is assigned level $\mathrm{B}$, as the $<<$ SafetyCritical $\gg>$ (5.2.17) stereotype illustrates, because its failure prevents the pilots from performing their tasks correctly and accurately, but the pilots can approximate the aircraft's navigation information by observing ground landmarks. This subsystem will not be considered any further in this case study.

4. LEDDisplaysubsystem: This subsystem represents a simple Light-Emitting Diode (LED) display to the pilots showing continuous navigation information as it is read from the "Navigation" subsystem. LEDs are a classical kind of information display technology. Thus, it is safety-critical and is assigned level D, as the $<<$ SafetyCritical $>>(5.2 .17)$ stereotype illustrates, because its failure increases the pilots' workload and discomfort, but they can still read the navigation information from NavigationUserInterfaceSubsystem. In case LEDDisplaySubsystem fails, they can approximate navigation information through ground landmarks or through radio communication with ground stations or other aircrafts. This subsystem will not be considered any further in this case study. 
5. NavigationDatabasesubsystem: This subsystem stores and manages all the possible flight paths relevant to this aircraft. It is safety-critical and is assigned level $\mathrm{C}$, as the $<<$ SafetyCritical $>$ (5.2.17) stereotype illustrates, because the NavigationControllersubsystem subsystem (at level "C") depends on it. This is a rule in the airworthiness standard - components are assigned to the highest level of the components whose operations depend on it. This subsystem will not be considered any further in this case study.

6. NavigationControllerSubsystem: This subsystem is in charge of automatically guiding the aircraft through pre-determined flight paths and FTPs. The pilots can use it for the autopilot feature, or to fly to specific points. It is safety-critical and is assigned level $\mathrm{C}$, as the $<<$ SafetyCritical $\gg$ (5.2.17) stereotype illustrates, because its failure increases the pilots' workload and discomfort and may cause injuries because the pilots will not necessarily be able to safely fly the aircraft without it as this would require them to use the mechanical steering wheels. It is partitioned away from Navigationsubsystem, as indicated by the $<<$ Partition $>>$ (5.2.18) stereotype, because it has a software level that is lower than that of Navigationsubsystem (i.e. level C is lower than level B). This partitioning allows NavigationSubsystem to continue providing information to other relevant subsystems such as LEDDisplaysubsystem even if NavigationControllerSubsystem fails. This subsystem is the topic of this case study, and is the only subsystem considered further here. 
7. NavigationUserInterfaceSubsystem: This subsystem serves as the pilots' interface to NavigationControllerSubsystem. It can be used to read navigation information including flight paths, and to command Navigationcontrollersubsystem to use the autopilot feature or to fly to specific FTPs. It is safety-critical and is assigned level C, as the $<<$ SafetyCritical $>>(5.2 .17)$ stereotype illustrates, because its failure increases the pilots' workload and discomfort and may cause injuries because the pilots will only be able to control the aircraft through Mechanicalsteeringwheelsubsystem. This subsystem will not be considered any further in this case study.

\subsection{Functional Requirements}

The following functional requirements are assigned to NavigationControllerSubsystem:

FREQ 1 NavigationControllerSubsystem shall be able to list pre-determined flight paths for a requested source/destination pair.

FREQ 2 NavigationControllerSubsystem shall provide an autopilot feature where it flies the aircraft through a requested flight path.

FREQ 3 NavigationControllersubsystem shall be able to fly the aircraft to a requested FTP. 
FREQ 4 NavigationControllerSubsystem shall provide the capability to guide the pilots through a requested flight path when the pilot is controlling the aircraft through Mechanicalsteeringwheelssubsystem.

FREQ 5 NavigationControllerSubsystem shall be able to provide navigation information received from Navigationsubsystem.

\subsection{Safety Assessment}

A safety assessment of NavigationControllersubsystem was performed based on the functional requirements listed in section 7.3 and the system architecture presented in Figure 12. This is common practice because such a safety assessment on the system helps identify potential hazards and their causes. This results in developing safety requirements to treat the hazards, which in turn impact the system and software design. Therefore, the safety assessment is performed at the early stages of the design because, unlike risk assessment, it emphasizes designing the system and software with safety in mind rather than adding safety features to a completed design.

The safety assessment in this case study was performed using various methods to identify additional safety-related requirements. Each one of the following sections (7.4.1 - 7.4.4) first presents and explains a safety assessment method. A description and analysis of the various safety assessment methods can be found in [1]. Several safety assessment methods are used in practice because they are complementary. Safety assessment methods differ in terms of inputs, outputs, objectives, and scalability. (Refer to section 2.1 for more detail.) Then, in each section, we apply the corresponding safety assessment 
method on the system, and only report on the results that are relevant to our case study (i.e., NavigationControllersubsystem).

Section 7.4.5 then presents safety requirements for NavigationControllerSubsystem based on the safety assessments performed in sections 7.4.1 - 7.4.4.

\subsubsection{Action Error Analysis (AEA)}

AEA is a qualitative safety-analysis technique used to analyse human behaviour and identify actions that can potentially cause accidents. It focuses on potential deviations of human behaviour from the normal or intended behaviour. Examples of scenarios considered in this analysis include forgetting to do a step, doing a step at the wrong time, incorrect ordering of steps, taking too long to do a step, or doing an unintended step. Therefore, it uses a forward search strategy to identify what could result from such errors in human behaviour. It is very similar to FMEA, but it is applied to human behaviour instead [1]. More detailed discussions of AEA can be found in [1] and [42].

The following potentially unsafe human actions, which could result in hazards, were analysed using this method:

AEA 1 A pilot attempts to manually control the aircraft through MechanicalsteeringWheelsubsystem while the aircraft is in autopilot mode.

AEA 2 A pilot requests flying to an FTP that is in an unsafe area. 
AEA 3 A pilot requests flying to an FTP where the path to it requires passing through an unsafe area.

AEA 4 A pilot requests a long flight path or a far FTP that would cause the aircraft to run out of fuel before landing.

\subsubsection{Failure Modes and Effects Analysis (FMEA)}

FMEA is a popular analysis technique, which was developed by reliability engineers. Therefore, it focuses on the failures of components and, using a forward search approach, analyses the effects of such failures. It is also used in safety assessments because the effects of such failures could include potential hazards and risks. When applied to safety assessments, however, it is important to realize that not all failures result in hazards or accidents. In addition, it pays little attention to human errors because it focuses on the failure of components. Therefore, FMEA and AEA complement each other. More detailed discussions of FMEA can be found in [1] and [43].

Using FMEA, failures that would result in the failure of NavigationControllerSubsystem were identified. Such failures could potentially result in hazards because NavigationControllersubsystem is safety-critical. They include the potential failure of NavigationControllersubsystem itself and any of the subsystems on which it depends.

The following failures, which could result in hazards, were identified using this method:

FMEA 1 NavigationControllerSubsystem fails. 
FMEA 2 WingsAndEnginesSubsystem fails.

FMEA 3 NavigationDatabaseSubsystem fails.

FMEA 4 Navigationsubsystem fails.

\subsubsection{Hazards and Operability Analysis (HAZOP)}

HAZOP is an analysis technique that assumes that accidents are caused by deviations from the design or operating intentions. Therefore, it encourages creative thinking about all the possible ways in which hazards or operating problems may arise as a result of using the system in a mode other than its intended operating conditions. Because HAZOP considers a design and investigates what hazards could be caused by each design and operating deviation, it can discover new hazards that were not previously identified. More detailed discussions of HAZOP can be found in [1] and [44].

The following deviation from the operating intention, which could result in hazards, was identified using this method:

HAZOP 1 The autopilot mode is being used when Navigationsubsystem is unable to use the GPS feature and is instead dead-reckoning (i.e. periodically approximating) the aircraft's position.

\subsubsection{Interface Analyses (IA)}

IA is an analysis method that is used to evaluate connections and relationships between components. It examines the interfaces between components and determines whether failures can be propagated between components. The types of problems that are often 
examined include, but are not limited to, failure to receive inputs from the connection, unstable connection, and erroneous output. IA is similar in use to HAZOP because interface problems are deviations from the intended design operation, but it is more general because it considers other types of problems. More detailed discussions of IA can be found in [1] and [45].

The following connection problems, which could result in hazards, were identified using this method:

IA 1 NavigationControllerSubsystem can no longer communicate with WingsAndEnginesSubsystem.

IA 2 NavigationControllersubsystem can no longer communicate with NavigationSubsystem.

\subsubsection{Safety Requirements}

The following safety requirements are assigned to NavigationControllersubsystem based on the results of the safety assessment performed above. Notice that sections 7.4.1 - 7.4.4 describe the events that could occur and the hazards that could result from them, whereas this section describes the positive reactions to those events, which would eliminate or reduce the hazards. The parenthesis specify the hazards that each safety requirement guards against:

SREQ 1 NavigationControllersubsystem shall disable autopilot and FTP features when the pilot is using MechanicalsteeringWheelsubsystem, 
and re-enable them when the pilot stops using MechanicalsteeringWheelsubsystem (AEA 1).

SREQ 2 NavigationControllerSubsystem shall be able to identify whether a specific LAT/LONG position is in a safe area or not, and not fly the aircraft to unsafe positions unless explicitly confirmed by the pilot (AEA 2).

SREQ 3 NavigationControllerSubsystem shall be able to determine whether flying to a specific LAT/LONG position requires flying through unsafe areas or not, and not fly the aircraft through unsafe areas unless explicitly confirmed by the pilot (AEA 3).

SREQ 4 NavigationControllerSubsystem shall alert the pilot when the next FTP cannot be reached without having to refuel the aircraft (AEA 4).

SREQ 5 When NavigationControllerSubsystem fails, an alert shall be raised and, until NavigationControllerSubsystem is operational again, the pilot shall be required to manually fly the aircraft using MechanicalsteeringWheelssubsystem (FMEA 1).

SREQ 6 NavigationControllerSubsystem shall ensure that the autopilot and FTP features are enabled only when all of the following conditions hold:

SREQ 6.1 WingsAndEnginesSubsystem is functional (FMEA 2).

SREQ 6.2 NavigationDatabasesubsystem is functional (FMEA 3). 
SREQ 6.3 Navigationsubsystem is functional (FMEA 4).

SREQ 6.4 NavigationControllerSubsystem is able to communicate with WingsAndEnginesSubsystem (IA 1).

SREQ 6.5 NavigationControllersubsystem is able to communicate with Navigationsubsystem (IA 2).

SREQ 7 NavigationcontrollerSubsystem shall require explicit confirmation to continue autopilot or FTP flight modes every 5 minutes until Navigationsubsystem indicates that the GPS feature is functional again. If the confirmation is not performed for a period of 7 consecutive minutes, then NavigationControllerSubsystem shall signal an emergency to the pilots (HAZOP 1).

\subsection{Subsystem Design}

This section further defines NavigationControllerSubsystem, and then it introduces its UML model. Sections 7.5.1 - 7.5.4 aim at further defining the subsystem by understanding its events of interest, and how the subsystem should react to those events. Section 7.5.1 explains how the events and reactions can be derived from the safety requirements. Section 7.5.2 defines the events that are of interest to the subsystem, and section 7.5.3 defines how the subsystem behaves, or reacts, in response to those events. Thus, they provide an event-reaction relationship that defines the system behaviour. To ensure that the system's behaviour is complete with respect to the events and reactions, the reactions are traced to the events that caused them. This is explained in section 7.5.4. 
Once the subsystem's behaviour is understood, its software design in presented in section 7.5.5. While the limited space in the diagrams makes it difficult to list all the possible stereotypes and tagged values that could be used, the safety information that is modeled is varied enough to show different kinds of safety information, stereotypes, and tagged values. It is important to note that, in practice, a UML modeling tool would allow the designers to specify as many stereotypes and tagged values while giving them the choice to show or hide specific stereotypes (or stereotype categories such as a particular profile's stereotypes) on diagrams while retaining the information in the tool's database. Furthermore, the model is also stereotyped according to Gomaa's class classification as presented in [7] and summarized in Appendix E.

The discussions in the sections below will often cross-reference events and reactions through their numbers, prefixed by either an " $E$ " for events or " $R$ " for reactions. Whenever an event is cross-referenced, the number between parenthesis represents its number as listed in section 7.5.2. Whenever a reaction is cross-referenced, similarly, the number between parenthesis represents its number as listed in section 7.5.3. For example, the Controllerfailed (E5) event is described in section 7.5.2, but the Disablecontroller $(\mathrm{R} 4)$ reaction is described in section 7.5.3.

\subsubsection{Identification of Events and Reactions}

To design safety into the system, it is important to identify all events (3.2.3.8) that could have safety implications, and the reactions (3.2.3.9) to those events. To identify the events, one needs to ask: Which inputs to the system, or changes in its state, should the system respond to because they may impact its safety? To identify the reactions, one 
needs to ask: How should the system behave when any of the identified events occurs? The answers to those questions are found in the safety requirements. For example, consider safety requirement SREQ 1 from section 7.4.5:

NavigationControllerSubsystem shall disable autopilot and FTP features
when the pilot is using MechanicalSteeringWheelSubsystem, and re-
enable them when the pilot stops using
MechanicalSteeringWheelSubsystem (AEA 1).

From this requirement, one can identify at least two events of interest: (1) The event of when the pilot starts using MechanicalsteeringWheelsubsystem; (2) The event of when the pilot stops using MechanicalsteeringWheelsubsystem. Also from this requirement, and from the identified events, one can identify at least the following reactions: (1) The reaction of disabling the autopilot and FTP features when the pilot starts using MechanicalsteeringWheelsubsystem; (2) The reaction of enabling the autopilot and FTP features when the pilot stops using Mechanicalsteeringwheelsubsystem.

\subsubsection{Events}

Figure 13 shows all the system events of interest. Each concrete class (i.e., leaf class in the generalization hierarchy) represents a unique event type, and an instantiation of a concrete event class represents a unique event. Each event class is stereotyped with $<<$ Event $>>(5.2 .15)$ to indicate that it is an event of interest, and its "EffectOnSafetyDirection" and "Context" tagged values are set where applicable.This figure is primarily used here to arrange events in a hierachy and therefore facilitate 
discussion and analysis. During design, these events may not necessarily tranlate into actual classes in the subsystem class diagram (and implementation).

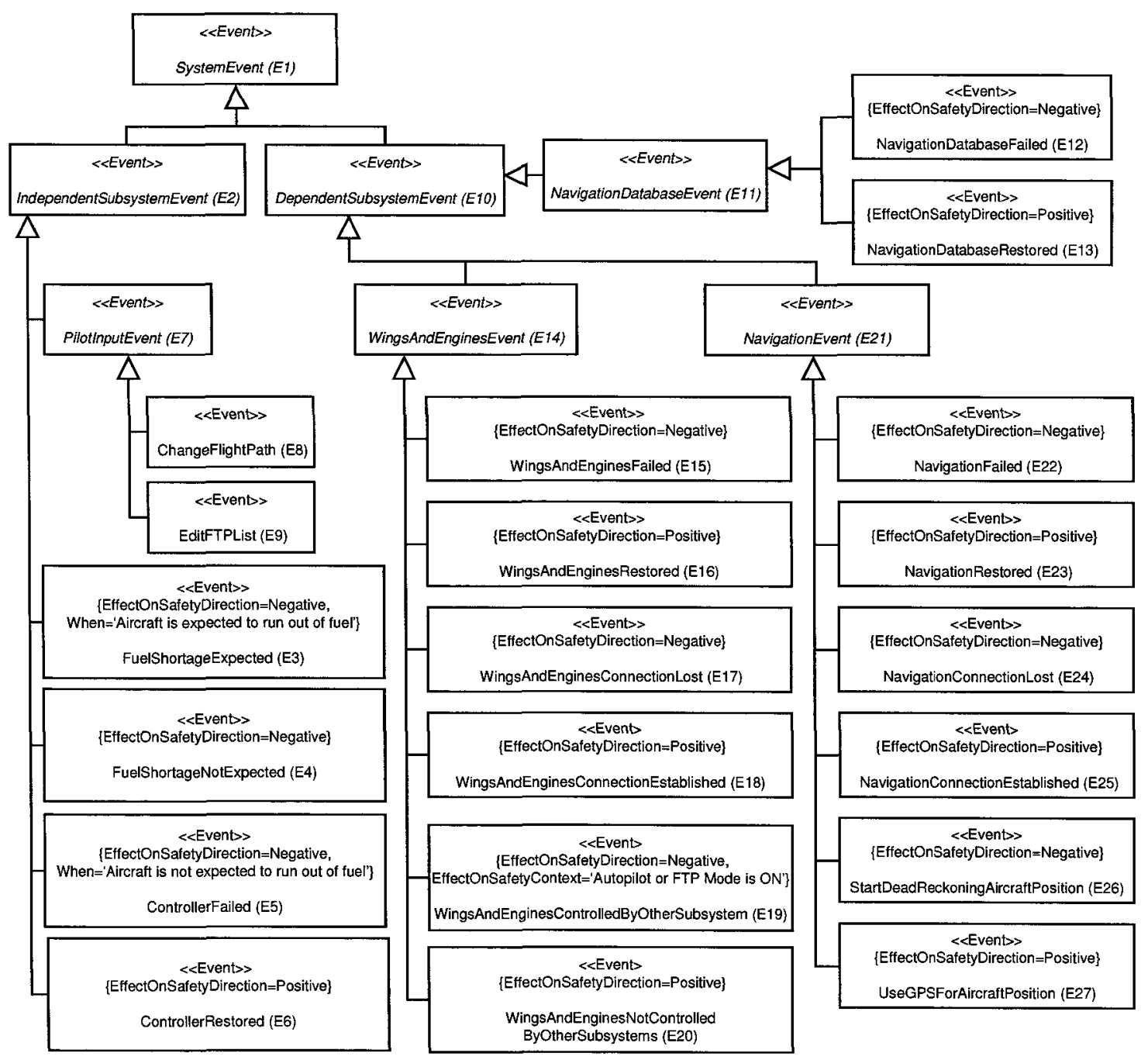

Figure 13: NavigationControllerSubsystem's events (structure).

Here is a description of each event class:

E1 Systemevent: This event represents any event that occurs in the system. It can be raised by any class in the system. It is abstract and it serves as a parent class for other event classes. A direct or indirect subclass of systemEvent may have a negative, neutral, or positive effect on the overall safety level. However, we 
limit the discussion here to only those events that can have a positive or negative effect on safety.

E2 IndependentSubsystemEvent: This event represents any event that originates in Navigationcontrollersubsystem, excluding the interface classes to other subsystems that it uses. It is abstract and it serves as a parent class for other event classes.

E3 FuelshortageExpected: This event is raised when the InvestigateFuelshortage (R6) reaction executes and it determines that the aircraft is expected to run out of fuel during the flight according to the current flight and navigation information (i.e. the flight path, list and sequence of FTPs, wind speed and bearing, ... etc).

E4 EuelshortageNotexpected: This event is raised when the InvestigateFuelshortage (R6) reaction executes and it determines that the aircraft is not expected to run out of fuel during the flight according to the current flight and navigation information. This is of importance when, just before the InvestigateFuelshortage (R6) reaction executes, the aircraft was expected to run out of fuel during the flight.

E5 Controllerfailed: This event is raised when the main controller class has failed and is not functioning correctly or at all.

E6 ControllerRestored: This event is raised when the main controller class has transitioned from a failure state to a functional state and is now functioning correctly.

E7 PilotInputEvent: This event represents any event that occurs as a direct result of the pilot's usage of the subsystem through the user interface, namely NavigationuserInterfacesubsystem. It is abstract and it serves as a parent class for other event classes. 
E8 ChangeFlightPath: This event is raised when the pilot has requested, through NavigationUserInterfacesubsystem, that the flight path for autopilot mode be changed.

E9 EditfTPList: This event is raised when the pilot has requested that, through NavigationUserInterfacesubsystem, the list of FTPs be changed (such as changing an FTP's position or resequencing a list of more than one FTP).

E10 DependentsubsystemEvent: This event represents any event that originates in a subsystem on which NavigationControllerSubsystem depends, namely WingsAndEnginesSubsystem, NavigationDatabasesubsystem, and Navigationsubsystem. It is abstract and it serves as a parent class for other event classes.

E11 NavigationDatabaseEvent: This event represents any event that originates in NavigationDatabaseSubsystem. It is abstract and it serves as a parent class for other event classes.

E12 NavigationDatabaseFailed: This event is raised when NavigationDatabasesubsystem has failed and is not functioning correctly or at all.

E13 NavigationDatabaseRestored: This event is raised when NavigationDatabaseSubsystem has transitioned from a failure state to a functional state and is now functioning correctly.

E14 WingsAndEnginesEvent: This event represents any event that originates in WingsAndEnginesSubsystem. It is abstract and it serves as a parent class for other event classes.

E15 WingsAndEnginesFailed: This event is raised when WingsAndEnginesSubsystem has failed and is not functioning correctly or at all. 
E16 wingsAndEnginesRestored: This event is raised when WingsAndEnginessubsystem has transitioned from a failure state to a functional state and is now functioning correctly.

E17 WingsAndEnginesConnectionLost: This event is raised when connection to WingsAndEnginesSubsystem has been lost.

E18 WingsAndEnginesconnectionEstablished: This event is raised when connection to WingsandEnginessubsystem was lost but has now been established.

E19 WingsAndEnginesControlledByOtherSubsystem: This event is raised when WingsAndEnginesSubsystem is now being controlled by a subsystem other than NavigationControllersubsystem. Based on the system architecture in Figure 12, this means that MechanicalsteeringWheelsubsystem is now controlling WingsAndEngines Subsystem.

E20 WingsAndEnginesNotControlledByOtherSubsystem: This event is raised when WingsandEnginessubsystem is no longer being controlled by a subsystem other than NavigationControllerSubsystem. Based on the system architecture in Figure 12, this means that Mechanicalsteeringwheelsubsystem has just stopped controlling WingsAndEnginesSubsystem.

E21 NavigationEvent: This event represents any event that originates in Navigationsubsystem. It is abstract and it serves as a parent class for other event classes.

E22 Navigationfailed: This event is raised when Navigationsubsystem has failed and is not functioning correctly or at all.

E23 NavigationRestored: This event is raised when Navigationsubsystem has transitioned from a failure state to a functional state and is now functioning correctly. 
E24 NavigationconnectionLost: This event is raised when connection to Navigationsubsystem has been lost.

E25 NavigationConnectionEstablished: This event is raised when connection to Navigationsubsystem was lost but has now been established.

E26 StartDeadReckoningAircraftPosition: This event is raised when the position of the aircraft is now being periodically estimated by the computer based on the knowledge of the current aircraft's position, speed, and bearing and the wind's speed and bearing. This occurs if the aircraft's Navigationsubsystem is no longer able to continuously determine the aircraft's position based on the GPS signals, most likely because it is no longer able to receive the GPS satelittes signals.

E27 UseGPSForAircraftPosition: This event is raised when the position of the aircraft is now being determined by the signals received from the GPS satelittes. This occurs when the aircraft's position was being dead-reckoned (see StartDeadReckoningAircraftPosition (E26) event), but the aircraft is now able to determine its position based on the GPS satellite signals, most likely because it is again able to receive the GPS satellite signals.

\subsubsection{Reactions}

Figure 14 shows all the system reactions to events of interest. Each class represents a unique reaction type, and an instantiation of a concrete reaction class (leaf class in the generalization hierarchy) represents a unique reaction. Each reaction class is stereotyped with $<<$ Reaction $>>(5.2 .16)$ to indicate that it is a reaction to an event of interest, and its tagged values are set where applicable. Like for events, the figure is primarily used to facilitate discussion and analysis. During design, these reactions will unlikely translate 
into actual subsystem classes, but rather will likely translate into class operations stereotyped $<<$ Reaction $>>$.

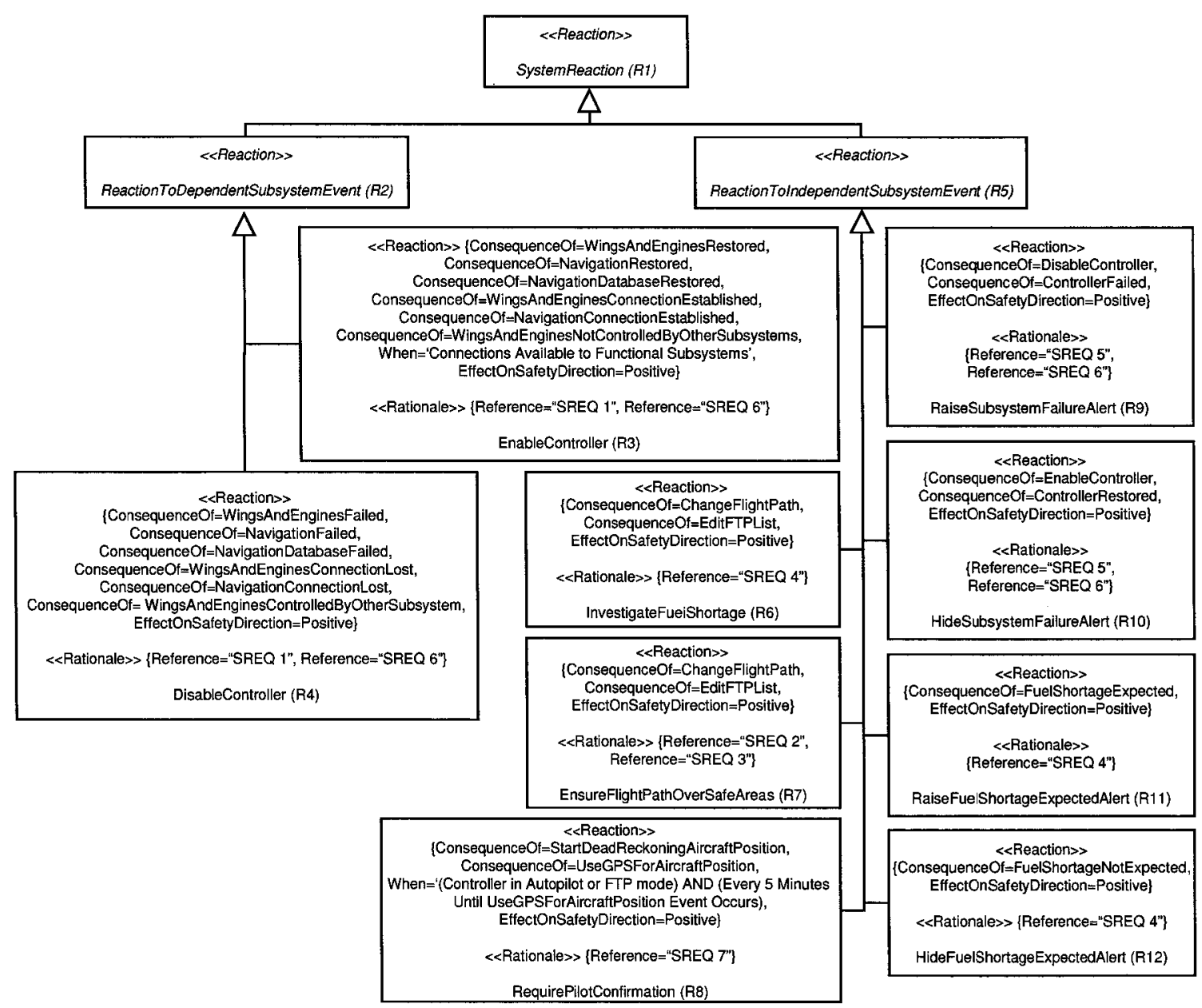

Figure 14: NavigationController subsystem reactions (structure).

Here is a description of each reaction class:

R1 SystemReaction: This represents any reaction that occurs in the system, which generally occurs in response to an event that is a subclass of SystemEvent (E1). It is abstract and it serves as a parent class for other reaction classes. A direct or indirect subclass of systemReaction may have negative, neutral, or positive effect on the overall safety level. However, all the concrete class reactions 
presented in this section have positive effect on safety, as indicated by the "EffectOnSafetyDirection" tagged value of the $<<$ Reaction $>>$ (5.2.16) stereotype, because they implement the safety requirements described in section 7.4.5.

R2 ReactionToDependentSubsystemEvent: This represents any reaction that occurs in response to an event class that is a subclass of the abstract DependentSubsystemEvent (E10) event. It is abstract and it serves as a parent class for other event classes.

R3 Enablecontroller: This reaction enables the main controller class to start functioning. As the "ConsequenceOf" tagged values of the $<<$ Reaction $>>$ (5.2.16) stereotype indicate, this reaction can be triggered by the occurrence of any of the following events: WingsAndEnginesRestored (E16), NavigationRestored (E23), NavigationDatabaseRestored (E13), WingsAndEnginesConnectionEstablished (E18), NavigationConnectionEstablished (E25), WingsAndEnginesNotControlledByOtherSubsystems (E20). Furthermore, the "When" tagged value indicates that this reaction (i.e. enabling the controller) only executes when connections are available to all functional subsystems on which the main controller class depends, namely WingsAndEnginesSubsystem, NavigationDatabasesubsystem, and Navigationsubsystem. The <<Rationale»> (5.2.14) stereotype and its "Reference" tagged values indicate that this reaction class helps implement safety requriements SREQ 1 and SREQ 6. 
R4 DisableController: This reaction disables the main controller class. As the "ConsequenceOf" tagged values of the $<<$ Reaction $>>$ (5.2.16) stereotype indicate, this reaction can be triggered by the occurrence of any of the following events: WingsAndEnginesFailed (E15), NavigationFailed (E22), NavigationDatabaseFailed (E12), WingsAndEnginesConnectionLost (E17), NavigationConnectionLost (E24), WingsAndEnginesControlledByOtherSubsystems (E19). The $<<$ Rationale $>$ (5.2.14) stereotype and its "Reference" tagged values indicate that this reaction class helps implement safety requriements SREQ 1 and SREQ 6.

R5 ReactionToIndependentSubsystemEvent: This represents any reaction that occurs in response to an event that is a subclass of the abstract Independent SubsystemEvent (E2) event. It is abstract and it serves as a parent class for other event classes.

InvestigateFuelshortage: This represents a reaction that calculates the fuel quantity needed to fly the aircraft according to the current settings, which include the aircraft's position, flight path, list of FTPs, and wind speed and bearing. As the "ConsequenceOf" tagged values of the $<<$ Reaction $>>$ (5.2.16) stereotype indicate, this reaction is triggered by either the ChangeFlightPath (E8) or EditfTPList (E9) events. The "Reference" tagged value of the $<<$ Rationale $>>(5.2 .14)$ stereotype indicates that this reaction implements safety requirement SREQ 4. If the execution of this reaction determines that the aircraft is expected to run out of fuel, then a FuelshortageExpected (E3) 
event is raised. Otherwise, a Fuel ShortageNotExpected (E4) event is raised. This is specified in the diagram using the "When" tagged value of the $<<$ Event»> (5.2.15) stereotype applied on the FuelShortageExpected (E3) and FuelshortageNotExpected (E4) events.

R7 EnsureFlightPathoverSafeAreas: This represents a reaction that ensures that the aircraft flies only over safe areas. As the "ConsequenceOf" tagged values of the $<<$ Reaction $\gg>(5.2 .16)$ stereotype indicate, this reaction is triggered by either the ChangeFlightPath (E8) or Editftplist (E9) events. The "Reference" tagged values of the $<<$ Rationale > (5.2.14) stereotype indicate that this reaction implements safety requirements SREQ 2 and SREQ 3.

R8 RequirePilotConfirmation: This represents a reaction that prompts the pilot to confirm the use of autopilot or FTP mode every 5 minutes as long as the pilot is in autopilot or FTP mode and the aircraft's position is being dead-reckoned instead of calculated using the GPS satellite signals, i.e. the UseGPSEOrAircraftPosition (E27) event has not been raised yet. As the "ConsequenceOf" tagged values of the $<<$ Reaction $>>$ (5.2.16) stereotype indicate, this reaction is triggered by two events, namely StartDeadReckoningAircraftPosition (E26) or UseGPSEorAircraftPosition (E27). The "Reference" tagged value of the $<<$ Rationale $>>$ (5.2.14) stereotype indicates that this reaction implements safety requirement SREQ 7.

R9 RaiseSubsystemfailureAlert: This represents a reaction that raises an alert to the pilots indicating that NavigationControllerSubsystem has failed, 
possibly because one of the subsystems on which it depends has failed as well. As the "ConsequenceOf" tagged values of the $<<$ Reaction $>>$ (5.2.16) stereotype indicate, this reaction is triggered by the Disablecontroller (R4) reaction or the controllerfailed (E5) event. The "Reference" tagged value of the $<<$ Rationale $>>$ (5.2.14) stereotype indicates that this reaction implements safety requirements SREQ 5 and SREQ 6.

R10 HideSubsystemFailureAlert: This represents a reaction that hides an alert, which was previously raised to the pilots as part of the RaiseSubsystemFailureAlert reaction, because NavigationControllerSubsystem has recovered from a previous failure. As the "ConsequenceOf" tagged values of the $<<$ Reaction $\gg>$ (5.2.16) stereotype indicate, this reaction is triggered by the Enablecontroller (R3) reaction or the ControllerRestored (E6) event. The "Reference" tagged values of the $<<$ Rationale $>>(5.2 .14)$ stereotype indicate that this reaction implements safety requirements SREQ 5 and SREQ 6.

R11 RaiseFuelShortageExpectedAlert: This represents a reaction that raises an alert to the pilots indicating that the aircraft is expected to run out of fuel before the final destination of the selected flight path or FTPs is reached. As the "ConsequenceOf" tagged value of the $<<$ Reaction $>>$ (5.2.16) stereotype indicates, this reaction is triggered by the FuelshortageExpected (E3) event. The "Reference" tagged values of the $<<$ Rationale»> (5.2.14) stereotype indicate that this reaction implements safety requirement SREQ 4. 
R12 HideFuelshortageExpectedAlert: This represents a reaction that hides an alert, which was previously raised to the pilots as part of the RaiseFuelShortageExpectedAlert (R11) reaction, because the flight path or FTPs have changed and it is not expected that they will cause fuel shortage anymore. As the "ConsequenceOf" tagged values of the $<<$ Reaction $>>(5.2 .16)$ stereotype indicate, this reaction is triggered by the FuelshortageNotExpected (E4) event. The "Reference" tagged values of the $<<$ Rationale $>$ (5.2.14) stereotype indicate that this reaction implements safety requirement SREQ 4.

\subsubsection{Event-Reaction Relationships}

A traceability matrix can be constructed as follows:

1. Identify the Events and Reactions: If the events (resp. reactions) are not explicitly identified and listed, then search for all model elements stereotyped with $<<$ Event $>>(5.2 .15)$ (resp. $<<$ Reaction $\gg>$ (5.2.16)). In general, only concrete events (resp. reactions) are those of interest here.

2. Create the Traceability Matrix: Create an $(\mathrm{N}+\mathrm{M}) \times \mathrm{M}$ matrix, where $\mathrm{N}$ is the number of unique events, and $\mathrm{M}$ is the number of unique reactions. Recall from section 3.2.3.9 that reactions are events by inheritance.

3. Identify Relationships: For every $<<$ Reaction $\gg>$ (5.2.16) stereotype, look at the "ConsequenceOf" tagged values. This tagged value identifies the events that can cause this reaction to occur. Therefore, this traces each reaction to the events that can cause it. 
4. Analyse Traceability: In general, each event must trigger at least one reaction. Each reaction may trigger zero or more reactions.

The relationships and traceability between the events described in section 7.5.2 and the reactions in section 7.5.3 are shown in Table 5 in the form of a traceability matrix, as described above. If a "Yes" exists in a particular cell, this means that the event identified by its row may trigger the reaction identified by its column. Only concrete events and reactions appear in the table.

\begin{tabular}{|c|c|c|c|c|c|c|c|c|c|c|}
\hline \multirow[t]{2}{*}{ Events } & \multicolumn{9}{|c|}{ Reactions } & \multirow[b]{2}{*}{ Total } \\
\hline & R3 & $\mathrm{R} 4$ & $\mathrm{R} 6$ & $\mathrm{R} 7$ & R8 & $\mathrm{R} 9$ & R10 & $\mathrm{R} 11$ & R12 & \\
\hline E3 & & & & & & & & Yes & & 1 \\
\hline E4 & & & & & & & & & Yes & 1 \\
\hline E5 & & & & & & Yes & & & & 1 \\
\hline E6 & & & & & & & Yes & & & 1 \\
\hline E8 & & & $\overline{\text { Yes }}$ & Yes & & & & & & 2 \\
\hline E9 & & & Yes & Yes & & & & & & 2 \\
\hline E12 & & Yes & & & & & & & & 1 \\
\hline E13 & Yes & & & & & & & & & 1 \\
\hline E15 & & Yes & & & & & & & & 1 \\
\hline E16 & Yes & & & & & & & & & 1 \\
\hline E17 & & Yes & & & & & & & & 1 \\
\hline E18 & Yes & & & & & & & & & 1 \\
\hline E19 & & Yes & & & & & & & & 1 \\
\hline E20 & Yes & & & & & & & & & 1 \\
\hline E22 & & Yes & & & & & & & & 1 \\
\hline $\mathrm{E} 23$ & Yes & & & & & & & & & 1 \\
\hline E24 & & Yes & & & & & & & & 1 \\
\hline E25 & Yes & & & & & & & & & 1 \\
\hline E26 & & & & & Yes & & & & & 1 \\
\hline E27 & & & & & & Yes & & & & 1 \\
\hline R3 & & & & & & & Yes & & & 1 \\
\hline R4 & & & & & & Yes & & & & 1 \\
\hline R6 & & & & & & & & & & 0 \\
\hline R7 & & & & & & & & & & 0 \\
\hline $\mathrm{R} 8$ & & & & & & & & & & 0 \\
\hline R9 & & & & & & & & & & 0 \\
\hline R10 & & & & & & & & & & 0 \\
\hline R11 & & & & & & & & & & 0 \\
\hline R12 & & & & & & & & & & 0 \\
\hline Total & 6 & 6 & 2 & 2 & 1 & 3 & 2 & 1 & 1 & 24 \\
\hline
\end{tabular}


Table 5: Relationships between events and reactions.

By analysing Table 5 we notice that there is an n-to-n relationship between events and reactions. More specifically:

1. Every event causes at least one reaction: Since all the reactions described in section 7.5.3 have a positive effect on safety, every event that may introduce hazards is being handled in a way that increases the level of safety by masking, reducing, or removing the hazard. Note, however, that reactions do not always cause other reactions to occur.

2. Every reaction can be triggered by at least one event: In essence, this means that none of the reactions will result in dead code that is never executed. In addition, a reaction's code may occur if any of a number of events occur (e.g. ChangeFlightPath (E8) occurs if either InvestigateFuelshortage (R6) or EnsureFlightPathoverSafeAreas (R7) occurs).

3. Some reactions are triggered by other reactions: the Rai sesystemFailureAlert (R9) reaction is triggered by the Disablecontroller (R4) reaction; the HideSystemFailureAlert (R10) reaction is triggered by the EnableController (R3) reaction.

4. Every event causes a finite number of reactions: In other words, it is guaranteed that the triggering of an event would eventually cause a reaction that does not trigger other reactions. If that was not the case, then the system could never restore itself to a steady-state. 
Note that we have initially defined our stereotypes (and associated tagged values) for a subset of the UML metamodel, specifically for metaclasses Class, Operation, and Relationship. The behavior suggested by these event-reaction relationships would, during a complete realistic design, also be modeled by one or several statechart diagrams. These statechart diagrams would specify which reactions (i.e., actions on transitions and states) result from which events (triggering transitions). The transitions' guard conditions would

then correspond to the "When" tagged value of the $<<$ Reaction $>>$ (5.2.16) stereotype above. This suggests that our UML profile needs to be extended to model elements of UML statechart diagrams. This will be considered in future work, and we do not foresee any major difficulty for doing so..

\subsubsection{High-Level Design}

Figure 15 illustrates the Navigationcontrollersubsystem high-level design, which contains safety information indicating requirements traceability, certification information, and safety monitoring. This will be elaborated further in the remainder of this section.

One key area of interest is tracing model elements, or classes, to requirements as described in section 2.5. This will be discussed in detail here as each classes contributing to the implementation of a requirement will be explained. The discussion will say that a class CLASS I implements requirement $R E Q 1$ if, as a minimum, class CLASSI partially implements requirement $R E Q 1$. 


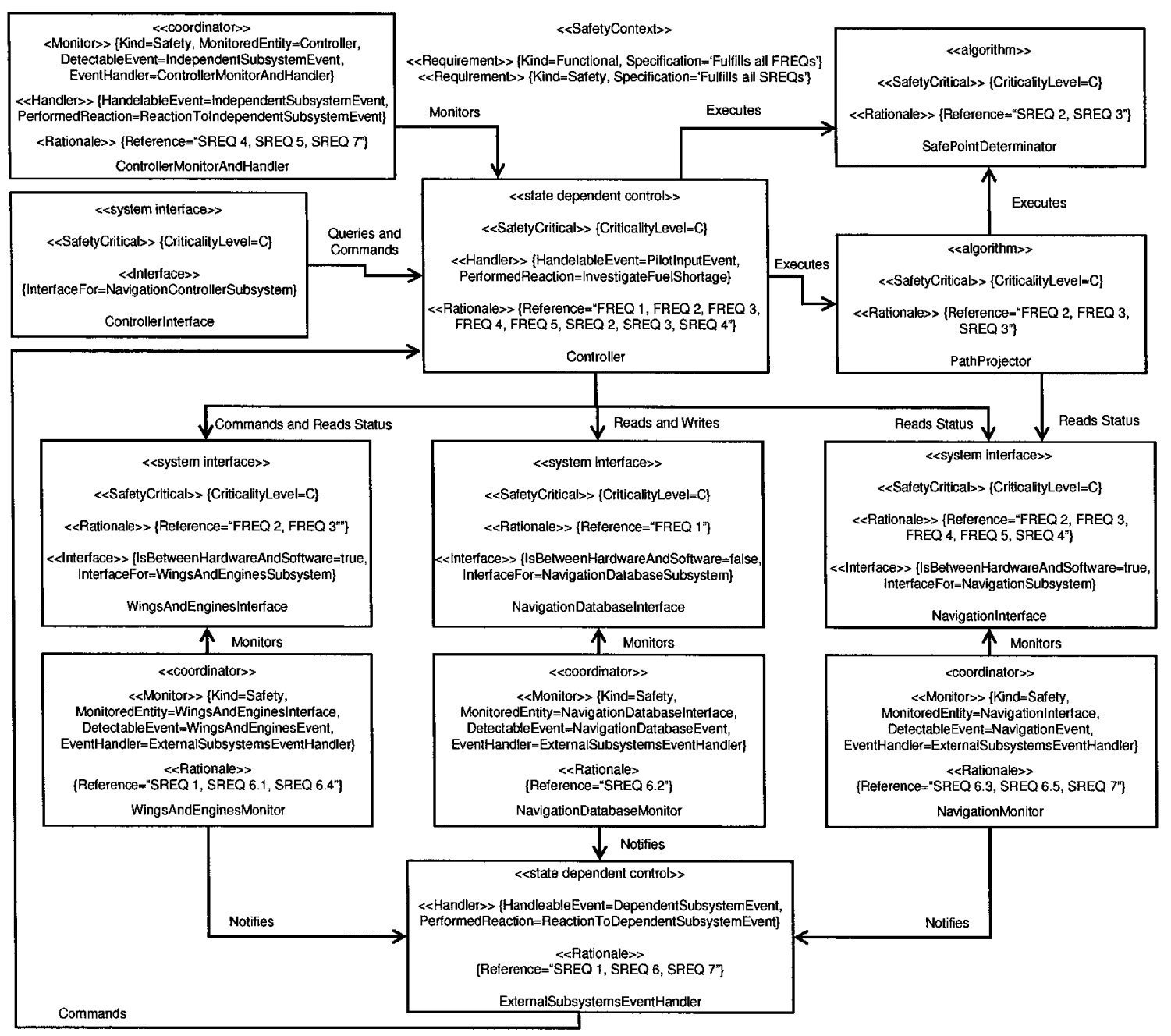

Figure 15: NavigationController subsystem's high-level design (structure).

The diagram is stereotyped with $<<$ SafetyContext $\gg>$ (5.2.1) to indicate that it contains information that is relevant to safety. Furthermore, it is also stereotyped with $<<$ Requirement>> (5.2.9) twice. The first $<<$ Requirement $>>$ (5.2.9) stereotype has a "Kind" tagged value equal to "Functional" to indicate that it is a functional requirement, and its "Specification" tagged value is set to "Fulfills all FREQs" to indicate that the design in this diagram must fulfill all the FREQ functional requirements of the subsystem (section 7.3). The second $<<$ Requirement $>>$ (5.2.9) stereotype has a "Kind" tagged value equal to "Safety" to indicate that it is a safety requirement, and its "Specification" tagged 
value is set to "Fulfills all SREQs" to indicate that the design in this diagram must fulfill all SREQ safety requirements of the subsystem (section 7.4.5).

Most classes in Figure 15 use the $<<$ Rationale $>$ (5.2.14) stereotype and its "Reference" tagged value. For clarity and brevity, the "Reference" tagged value was used once to list more than one requirement. For example, controller has a $<<$ Rationale $>$ (In 5.2.14) stereotype with a "Reference" tagged value set to "FREQ 1, FREQ 2, FREQ 3, FREQ 4, FREQ 5, SREQ 2, SREQ 3". This abbreviation is used in this, we say that a class CLASS1 depends on class CLASS2 if there is an association between CLASS1 and CLASS2 in which CLASS1 is the source end of the uni-directional association and CLASS2 is the target end. Additionally, we say that CLASS1 depends on CLASS2 if a usage dependency exists for CLASS1 on CLASS2, which would be the case study to indicate that the sterotype actually has several "Reference" tagged values, and each "Reference" tagged value identifies only if CLASS2 is a parameter to at least one requirement. Therefore, the $\{$ Reference="FREQ 1, FREQ 2, FREQ 3, FREQ 4, FREQ 5, SREQ 2, SREQ 3"' string is actually identical to Reference="FREQ 1", Reference="FREQ 2", Reference="FREQ 3", Reference="FREQ 4", Reference="FREQ 5", Reference="SREQ 2", Reference="SREQ 3"\}. Whereas the latter format is what the proposed UML profile defines and therefore would be used when the design is modeled in a UML modeling tool, we have used the former abbreviated format in the figuremethod in this section to make the UML diagram clearer and easier to understand.

Similarly, the "Explanation" tagged value of the $<<$ Rationale $>>(5.2 .14)$ stereotype is not shown in Figure 15 because the explanation text is large and would have cluttered the diagram. Instead, the explanation can be found below in the subsections describing each 
class: each time we provide the text (i.e., the value of the "Explanation" tagged value) that would be used by a UML CASE tool supporting our profile.

The different classes in the class diagram of Figure 15 are now described in sections 7.5.5.1 to 7.5.5.10.

\subsubsection{Description of class Controller}

Controller is the key and central element of the subsystem. It is stereotyped with < SafetyCritical> (5.2.17) and assigned software level C, as indicated by the "CriticalityLevel" tagged value, because its failure results in the failure of the entire subsystem, which is assigned software level C itself. Furthermore, controller is stereotyped with $<<$ Handler $>>$ (5.2.19) whose "HandleableEvent" tagged value is set to "PilotInputEvent" to indicate that it handles all concrete events that are subclasses of PilotinputEvent (E7). In addition to normal code execution (e.g. changing the flight path in response to a ChangeflightPath (E8) input event), controller also executes the InvestigateFuelShortage (R6) reaction to decide whether the changes requested by the pilots will result in a fuel shortage or not. This reaction results in sending one of two events (discussed later): FuelshortageExpected (E3) and FuelShortageNotExpectedEvent (E4). There is not a separate monitor shown here, using the $<<$ Monitor $>$ (5.2.20) stereotype, because those events are explicit invocation calls through NavigationInterface.

WingsAndenginesInterface is NavigationControllerSubsystem's interface to WingsAndEnginesSubsystemSubsystem. Therefore, it is stereotyped with <<Interface > (5.2.25). Its "IsBetweenHardwareAndSoftware" tagged value is set to "true" to indicate 
the class interfaces directly with hardware, and its "InterfaceFor" tagged value is set to WingsAndEnginesSubsystemSubsystem to specify the subsystem for which this class is an interface for.

Controller implements all the functional requirements assigned to the subsystem, namely FREQ 1, FREQ 2, FREQ 3, FREQ 4, and FREQ 5. This is explicitly indicated in the diagram by stereotyping controller with $<<$ Rationale $>$ (5.2.14) and identifying the functional requirements through the "Reference" tagged value. Furthermore, Controller also implements three safety requirements, namely SREQ 2, SREQ 3, and SREQ 4, which are also listed in the "Reference" tagged value of the $<<$ Rationale $>>$ (5.2.14) stereotype. The "Explanation" tagged value for each requirement is:

1. FREQ 1: To implement this functional requirement, controller uses NavigationDatabaseInterface (see section 7.5.5.10) to read pre-determined flight paths from NavigationDatabasesubsystem.

2. FREQ 2: To implement this functional requirement, Controller needs to provide an autopilot functionality, which requires continuously controlling the aircraft's wings and engines through wingsAndEnginesInterface, as well as using NavigationInterface to read the navigation parameters and determine the correct input parameters to the wings and engines (section 7.5.5.10). If the requested flight path is not pre-determined (i.e. loaded from the navigation database), then Controlier uses PathProjector (section 7.5.5.9) to determine the most appropriate flight path based on the pilots' input parameters. 
3. FREQ 3: To implement this functional requirement, controller needs to be able to fly the aircraft to a specific FTP, which requires continuously controlling the aircraft's wings and engines through wingsAndEnginesInterface, as well as using NavigationInterface to read the navigation parameters (section 7.5.5.10). To determine the flight path from the aircraft's current position to the requested FTP, Controller uses PathProjector to determine what the most appropriate flight path based on the pilots' requested FTP (section 7.5.5.9).

4. FREQ 4: To implement this functional requirement, controller needs to be able to guide the pilots with navigation directions by tracking the aircraft's position and bearing, following the flight path, without controlling the aircraft's wings and engines through WingsAndEnginesInterface. In this mode, the pilot manually controls the aircraft through Mechanicalsteeringwheelsubsystem. However, Controller uses NavigationInterface to read the navigation parameters and be able to provide correct flight guidance to the pilots (section 7.5.5.10). This operating mode requires controller to calculate the required inputs to WingsAndEnginesSubsystem, which is then displayed to the pilots. Such calculations take various factors into account, including the aircraft's navigation information, wind navigation information, and unsafe areas.

5. FREQ 5: To implement this functional requirement, controller needs to be able to read the navigation information (e.g., aircraft's and the wind's position and speed) from Navigationsubsystem (section 7.5.5.10). 
6. SREQ 2: To implement this safety requirement, controller needs to be able to execute an algorithm that determines whether a specific LAT/LONG position is in safe area or not. This is the responsibility of SafePointDeterminator (section 7.5.5.8).

7. SREQ 3: To implement this safety requirement, controller needs to be able to execute an algorithm that projects a flight path based on the current aircraft's position and the target FTP, and then determines whether this path includes flying in unsafe areas. This algorithm is the responsibility of PathProjector (section 7.5.5.9). Once a path is determined, then an algorithm in SafePointDeterminator is executed to determine whether the flight path is safe or not (section 7.5.5.8).

8. SREQ 4: To implement this safety requirement, controller needs to be able to investigate whether a fuel shortage is expected, based on the current navigation information and flight information, or not. The navigation information is available through NavigationInterface (section 7.5.5.10). The determination of whether fuel shortage is expected or not occurs in the execution of the InvestigateFuelShortage (R6) reaction. As a result of this execution, it raises either the FuelShortageExpected (E3) event or the FuelShortageNotExpected (E4) event. Raising an event is the explicit indication that a particular event has occurred, which would normally result in the detection of the event and its appropriate handing by executing the corresponding reactions. The event is then detected and handled by ControllerMonitorAndHandler (section 7.5.5.6). 


\subsubsection{Description of class wingsAndEnginesMonitor}

WingsAndEnginesMonitor is a safety monitoring class as indicated by the $<<$ Monitor $>$ (5.2.20) stereotype and its "Kind" tagged value, which is set to "Safety". The purpose of this class is to continuously monitor WingsAndEnginessubsystem through WingsAndEnginesInterface to detect any event that may impact safety. This is specified in the "MonitoredEntity" tagged value, which is set to "WingsAndEnginesInterface" because, as far as NavigationControllerSubsystem is concerned, WingsAndEnginesInterface is the single point of interface with WingsAndEnginesSubsystem. The safety-related events that wingsAndEnginesMonitor detects are specified in the "DetectableEvent" tagged value, which is set to "WingsAndEnginesEvent" to indicate that it detects all events of type WingsAndEnginesEvent and its subclasses. Furthermore, the "EventHandler" tagged value is set to "ExternalSubsystemsEventHandler" to indicate that ExternalsubsystemsEventHandler is the event handler for the events that WingsAndEnginesMonitor detects.

The requirements that wingsAndEnginesMonitor implements are indicated by the "Reference" tagged value of the $<<$ Rationale $>>$ (5.2.14) stereotype, namely SREQ 1 , SREQ 6.1, and SREQ 6.4. The "Explanation" tagged values of stereotype $<<$ Rationale $>>$ are:

1. SREQ 1: It implements this safety requirement by monitoring WingsAndEnginesInterface for the following two events:

and 
WingsAndEnginesNotControlledByOtherSubsystem (E20). If any of them is detected, ExternalSubsystemsEventHandler is notified accordingly to handle the event (section 7.5.5.5).

2. SREQ 6.1: It implements this safety requirement by monitoring WingsAndEnginesInterface for the WingsAndEnginesFailed (E15) and WingsAndEnginesRestored (E16) events. If any of them is detected, ExternalSubsystemsEventHandler is notified accordingly to handle the event (section 7.5.5.5).

3. SREQ 6.4: It implements this safety requirement by monitoring WingsAndEnginesInterface for the WingsAndEnginesConnectionLost (E17) and WingsandEnginesConnectionEstablished (E18) events. If any of them is detected, ExternalsubsystemsEventHandler is notified accordingly to handle the event (section 7.5.5.5).

\subsubsection{Description of class NavigationDatabaseMonitor}

NavigationDatabasemonitor is a safety monitoring class as indicated by the <<Monitor>> (5.2.20) stereotype and its "Kind" tagged value, which is set to "Safety". The purpose of this class is to continuously monitor NavigationDatabasesubsystem through NavigationDatabaseInterface to detect any events that may impact safety. This is specified in the "MonitoredEntity" tagged value, which is set to "NavigationDatabaseInterface" because, as far as NavigationControllerSubsystem is concerned, NavigationDatabaseInterface is the single point of interface with NavigationDatabaseSubsystem. The safety-related events that NavigationDatabase- 
Monitor detects are specified in the "DetectableEvent" tagged value, which is set to "NavigationDatabaseEvent" to indicate that it detects all events of NavigationDatabaseEvent and its subclasses. Furthermore, the "EventHandler" tagged value is set to "ExternalSubsystemsEventHandler" to indicate that ExternalsubsystemsEventHandler is the event handler for the events that NavigationDatabasemonitor detects.

The requirements that NavigationDatabasemonitor implements are indicated by the "Reference" tagged value of the $<<$ Rationale $>$ (5.2.14) stereotype, namely SREQ 6.2. The detectable events are NavigationDatabaseFailed (E12) and NavigationDatabaseRestored (E13). If any of them is detected, ExternalsubsystemsEventHandler is notified accordingly to handle the event (section 7.5.5.5). The "Explanation" tagged value of the $<<$ Rationale $>>(5.2 .14)$ stereotype is exactly the explanation provided in this paragraph.

\subsubsection{Description of class NavigationMonitor}

NavigationMonitor is a safety monitoring class as indicated by the $<$ Monitor $>$ (5.2.20) stereotype and its "Kind" tagged value, which is set to "Safety". The purpose of this class is to continuously monitor Navigationsubsystem through NavigationInterface to detect any events that may impact safety. This is specified in the "MonitoredEntity" tagged value, which is set to "NavigationInterface" because, as far as NavigationControllersubsystem is concerned, NavigationInterface is the single point of interface with Navigationsubsystem. The safety-related events that NavigationMonitor detects are specified in the "DetectableEvent" tagged value, which 
is set to "NavigationEvent" to indicate that it detects all events of NavigationEvent and its subclasses. Furthermore, the "EventHandler" tagged value is set to "ExternalSubsystemsEventHandler" to indicate that ExternalSubsystemsEventHandler is the event handler for the events that NavigationMonitor detects. This is an example of using the same class as both a monitor and an event handler, which may be more appropriate in cases where the events and reactions are relatively simple, possibly because the "MonitoredEntity" is a single class, namely, controller. Each of the other monitors in the subsystem monitors an entire subsystem, through its interface. Those monitored subsystems reside on different nodes, and therefore there are several other factors that monitors need to be aware of, such as communication through data buses.

The requirements that NavigationMonitor implements are indicated by the "Reference" tagged value of the $<<$ Rationale $>$ (5.2.14) stereotype, namely SREQ 6.3, SREQ 6.5, and SREQ 7. The "Explanation" tagged values of the $<<$ Rationale $>>$ (5.2.14) stereotype are:

1. SREQ 6.3: It implements this safety requirement by monitoring NavigationInterface for the Navigationfailed (E22) and NavigationRestored (E23) events.

2. SREQ 6.5: It implements this safety requirement by monitoring NavigationInterface for the NavigationconnectionLost (E24) and NavigationConnectionEstablished (E25) events.

3. SREQ 7: It implements this safety requirement by monitoring NavigationInterface for the StartDeadReckoningAircraftPosition (E26) and UseGPSForAircraftPosition (E27) events. 
If any of these events is detected, ExternalsubsystemsEventHandler is notified accordingly to handle the event (section 7.5.5.5).

\subsubsection{Description of class ExternalSubsystemsEventHandler}

ExternalSubsystemsEventHandler is an event handler as indicated by the $<<$ Handler $>$ (5.2.19) stereotype. Thus, the "HandleableEvent" tagged value is set to "DependentSubsystemEvent", which is the event class, and its subclasses, that ExternalSubsystemsEventHandler can recognize and handle. All the events that are passed to ExternalSubsystemsEventHandler from WingsAndEnginesMonitor, NavigationDatabaseMonitor, or NavigationMonitor are subclasses of the Dependent SubsystemEvent (E10).

The reactions that ExternalSubsystemsEventHandler performs in response to those events are the concrete subclasses of ReactionToDependentsubsystemEvent (R2) and its subclasses. The "Reference" tagged value of the <<Rationale»> (5.2.14) stereotype specifies the requirements that ExternalSubsystemsEventHandler fulfills by performing the reactions to the event, namely ${ }^{1}$ SREQ 1, SREQ 6 (and its sub-requirements), and SREQ 7. Specifically, it implements each requirement by invoking the corresponding reaction for each event it is notified with. As before, the "Explanation" tagged value of the $<<$ Rationale > (5.2.14) stereotype is not shown in the diagram because it is large and would have cluttered the diagram, but the explanation provided in this paragraph is

\footnotetext{
' WingsAndEnginesMonitor, NavigationDatabaseMonitor, and NavigationMonitor send events that are handled by ExternalsubsystemsEventHandler to implement requirements SREQ 1, SREQ 6.1, and SREQ 6.4 (WingsAndEnginesMonitor-section 7.5.5.2), SREQ 6.2 (NavigationDatabaseMonitor-section 7.5.5.3), SREQ 6.3, SREQ 6.5, and SREQ 7 (NavigationMonitor-section 7.5.5.4).
} 
actually the value of the "Explanation" tagged value and is what would be captured in a UML tool.

\subsubsection{Description of class ControllerMonitorAndHandler}

ControllerMonitorAndHandler is a safety monitoring class that monitors controller. Unlike the monitoring classes discussed above, this class is also the handler for the events it detects. It was a design decision to combine those two functionalities in one class because all of the events detected, and reactions performed, by this class are simple enough to combine both in one class. On the other hand, WingsAndEnginesMonitor, NavigationDatabaseMonitor, and NavigationMonitor are designed to detect more complex events. Those complex events require the monitors to continuously interact with the subsystems they monitor by sending and receiving inter-subsystem messages through their interface classes.

The $<<$ Monitor $>$ (5.2.20) stereotype on ControllerMonitorAndHandler explicitly indicates that the class is a monitor, and the "Kind" tagged value, which is set to "Safety", indicates that its purpose is to monitor safety. The "MonitoredEntity" tagged value is set to "Controller" to indicate ControllerMonitorAndHandler monitors Controller for safety-related events. The "DetectableEvent" tagged value is set to "IndependentSubsystemEvent" to indicate that it detects all events of IndependentSubsystemEvent and its subclasses. Furthermore, the "EventHandler" tagged value is set to "ControllerMonitorAndHandler" to indicate that ControllerMonitorAndHandler itself is the event handler for the events that it detects. 
The $\quad<$ Handler $>\quad(5.2 .19) \quad$ stereotype explicitly indicates that ControllermonitorAndHandler also handles the events that it detects. The "HandleableEvents" tagged value is set to "IndependentSubsystemEvent" to indicate that the class can handle all IndependentSubsystemEvent events and its subclasses. Furthermore, the "PerformedReaction" tagged value is set to "ReactionToIndependentSubsystemEvent" to indicate that ControllermonitorAndHandler performs all reactions of ReactionToIndependent SubsystemEvent and its subclasses in response to the events.

ControllerMonitorAndHandler is also stereotyped with $<<$ Rationale $>$ (5.2.14) whose "Reference" tagged value is set to "SREQ 4, SREQ 5, SREQ 7". The "Explanation" tagged values of the $<<$ Rationale $>$ (5.2.14) stereotype are:

1. SREQ 4: It implements this safety requirement by monitoring controller for the FuelShortageExpected (E3) and FuelshortageNotExpected (E4) events (section 7.5.5.1). Since ControllerMonitorAndHandler is also an event handler, it executes the RaiseFuelShortageExpectedAlert (R11) reaction in response to the FuelshortageExpected (E3) event, and the HideFuelshortageExpectedAlert (R12) reaction in response to the FuelShortageNotExpected (E4) event.

2. SREQ 5: It implements this safety requirement by monitoring control ler for the ControllerFailed (E5) and ControllerRestored (E6) events (section 7.5.5.1). It is ControllerMonitorAndHandler that creates those events based on how it observes the behaviour of controller to be. Since 
ControllerMonitorAndHandler is also an event handler, it executes the RaiseSubsystemFailureAlert (R9) reaction in response to the FuelShortageExpected (E5) event, and the HideSubsystemFailureAlert (R10) reaction in response to the ControllerRestored (E6) event.

3. SREQ 7: It implements this safety requirement by monitoring controller, and by being sensitive to the StartDeadReckoningAircraftPosition (E26) and UseGPSForAircraftPosition (E27) events (section 7.5.5.1). Since ControllerMonitorAndHandler is also an event handler, it executes the RequirepilotConfirmation (R8) reaction, which periodically requires the pilot's confirmation as long as the aircraft's position is being dead-reckoned, in response to the StartDeadReckoningAircraftPosition (E26) and UseGPSE orAircraftPosition (E27) events.

\subsubsection{Description of class controllerinterface}

ControllerInterface serves as the façade of NavigationControllersubsystem to the other subsystems. This has two implications. First of all, it is safety-critical and has a software level equal to that of NavigationControllersubsystem. This is indicated by stereotyping it with $<<$ SafetyCritical $>>$ (5.2.17) and setting its "CriticalityLevel" tagged value to "C". Secondly, it is stereotyped with $<<$ Interface $>>$ (5.2.25) and has its "InterfaceFor" tagged value set to "NavigationControllerSubsystem" to indicate that it serves as an interface for NavigationControllersubsystem. The services this interface provides is quering controller, and consequently other classes that it depends on, and commanding controller to perform certains functionalities such as activating autopilot 
or FTP mode. Notice that ControllerInterface is not stereotyped with $<<$ Rationale $>$ (5.2.14) in this diagram because it is controller that implements the functional requirements. In the class diagram for some other subsystem, such as NavigationUserInterfaceSubsystem, ControllerInterface serves as the façade of NavigationcontrollerSubsystem and its classes, including Controller. In that context, it is likely to include controllerInterface and stereotype it with $<<$ Rationale $>>$ (5.2.14) and include all the FREQ ans SREQ requirements in its "Reference" tagged value. This is what was done here for the interfaces for other subsystems (WingsAndEnginesInterface, NavigationDatabaseInterface, and NavigationInterface).

\subsubsection{Description of class SafePointDeterminator}

SafePointDeterminator implements an algorithm that determines whether a specific LAT/LONG position is in a safe area or not. Similarly, it implements an algorithm that determines whether a flight path is safe or not. These services are provided to Controller to implement requirements SREQ 2 and SREQ 3 (see section 7.5.5.1). Therefore SafePointDeterminator participates in the implementation of this requirement and is stereotyped with $<<$ Rationale $>>$ (5.2.14) whose "Reference" tagged value includes "SREQ 2" and "SREQ 3". 
Additionally, SafePointDeterminator is stereotyped with $<<$ SafetyCritical $>>$ (5.2.17) whose "CriticalityLevel" tagged value is set to "C" because ${ }^{2}$ it is used by controller which is itself safety critical at level " $\mathrm{C}$ ".

\subsubsection{Description of class PathProjector}

PathProjector provides services to controller, such as obtaining the source and destination LAT/LONG positions, specific intermediate flight points, $\ldots$ of a path, when Controller does not have a pre-determined flight path (see section 7.5.5.1). Therefore, PathProjector participates in implementing requirement FREQ 2. Similarly, PathProjector participates in implementing requirement FREQ 3 (see section 7.5.5.1). Additionally, PathProjector implements an algorithm that projects a flight path based on the current aircraft's position and the target FTP. This service is also used by Controller to implement requirement SREQ 3. PathProjector is therefore stereotyped with <<Rationale > (5.2.14) whose "Reference" tagged value includes "FREQ 2", "FREQ 3", and "SREQ 3".

Additionally, PathProjector is stereotyped with $\ll<$ SafetyCritical $>$ (5.2.17) whose "CriticalityLevel" tagged value is set to "C" because ${ }^{2}$ it is used by controller which is itself safety critical at level "C".

\footnotetext{
${ }^{2}$ According to the rules for determining software levels described in the airworthiness standard RTCA DO178B [4], when a safety critical component has a specific criticality level, then all the components it depends on are safety critical and have at least this criticality level (' $\mathrm{A}$ ' is more critical than ' $\mathrm{B}$ ', which is more critical than ' $\mathrm{C}$ ', ...). In our case, components are classes, and a class depends on another class if the former class requires services from the latter class, which is specified in the class diagram as an association that can be navigated from the former to the latter.
} 
7.5.5.10 Description of WingsAndEnginesInterface, NavigationDatabaseInterface, and NavigationInterface classes

Finally, the class diagram for Navigationcontrollersubsystem includes three interface classes that communicate with other subsystems. For each one of those interface (client) classes, there exists a server class in the corresponding subsystem that communicates with it by receiving messages, usually known as requests in such distributed systems, from the interface and then responding to them. Those interface classes are:

1. WingsAndEngines Interface, which belongs to WingsAndEnginesSubsystem. It is NavigationControllersubsystem's interface to WingsAndEnginessubsystem. Therefore, it is stereotyped with $<<$ Interface $>>$ (5.2.25). Its "IsBetweenHardwareAndSoftware" tagged value is set to "true" to indicate the class interfaces directly with hardware, and its "InterfaceFor" tagged value is set to WingsAndEnginesSubsystemsubsystem to specify the subsystem for which this class is an interface for. WingsAndEnginesInterface is stereotyped with $<<$ Rationale $>>$ (5.2.14) whose "Reference" tagged value includes "FREQ 2" and "FREQ 3" because it is used by class controller to implement these requirements (see section 7.5.5.1).

2. NavigationDatabaseInterface, which belongs to the NavigationDatabaseSubsystem. It is NavigationControllerSubsystem's interface to NavigationDatabaseSubsystem. Therefore, it is stereotyped with <Interface > (5.2.25). Its "IsBetweenHardwareAndSoftware" tagged value is set 
to "false" to indicate the class does not interface with hardware, and its "InterfaceFor" tagged value is set to "NavigationDatabaseSubsystem" to specify the subsystem for which this class is an interface for. NavigationDatabaseInterface is stereotyped with $<<$ Rationale $>$ (5.2.14) whose "Reference" tagged value includes "FREQ 1" because it is used by class Controller to implement this requirement (see section 7.5.5.1).

3. NavigationInterface, which belongs to the Navigationsubsystem. It is NavigationControllerSubsystem's interface to NavigationSubsystem. Therefore, it is stereotyped with $<<$ Interface $>>$ (5.2.25). Its "IsBetweenHardwareAndSoftware" tagged value is set to "true" to indicate the class interfaces directly with hardware, and its "InterfaceFor" tagged value is set to "NavigationSubsystem" to specify the subsystem for which this class is an interface for. NavigationInterface is stereotyped with $<<$ Rationale $>$ (5.2.14) whose "Reference" tagged value includes "FREQ 2", "FREQ 3", "FREQ 4", "FREQ 5", and "SREQ 4" because it provide navigation parameters, such as the aircraft's and the wind's bearing and speed, the aircraft's and the wind's position and speed, to the controller class to implement these requirements (see section 7.5.5.1)

Last, these three classes are stereotyped with $<$ SafetyCritical $\gg$ (5.2.17) whose "CriticalityLevel" tagged value is set to "C" because ${ }^{2}$ they are used by controller which is itself safety critical at level "C". 


\subsubsection{Low-Level Design of Events and Reactions}

There are several possible ways to design and implement the events and reactions in software. One approach would be to design them as classes. In this case, the class diagram for each the events would be exactly the one shown in Figure 13, and the class diagram for reactions would be exactly the one shown in Figure 14. A concrete event instance is simply an instantiation of its corresponding class. This object instance would then be passed from a monitor, stereotyped with $\ll$ Monitor $>(5.2 .20)$, to a handler $<$ Handler $>$ (5.2.19). A reaction would also be an instantiation of a reaction class. Executing it would simply correspond to passing an event object instance to a procedure in the reaction object instance. For example, here is a sample code for ControllerMonitorAndHandler illustrating how reactions to the ChangeFlightPath (E8) event are executed:

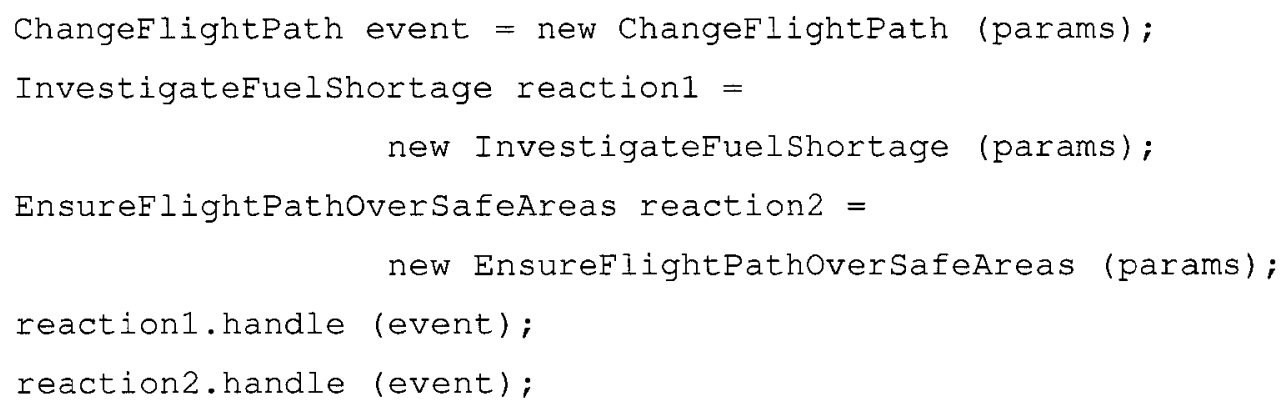

However, it is not necessary to have a separate class for each reaction. In fact, only one procedure, in a Handler (3.2.3.12) class, is generally needed to describe and execute a reaction instead of an entire class, although this is dependent on the application details and the sizes of the events and reactions (i.e. it is up to the designers to ensure that events and reactions are appropriately defined). Therefore, it is often feasible to group all related reaction procedures together and include them in the class that is stereotyped with 
$<<$ Handler $>$ (5.2.19). That way, the handler does not need to keep track on which reactions should occur in response to which events. This would simplify the design and implementation. Here is a sample code illustrating this concept:

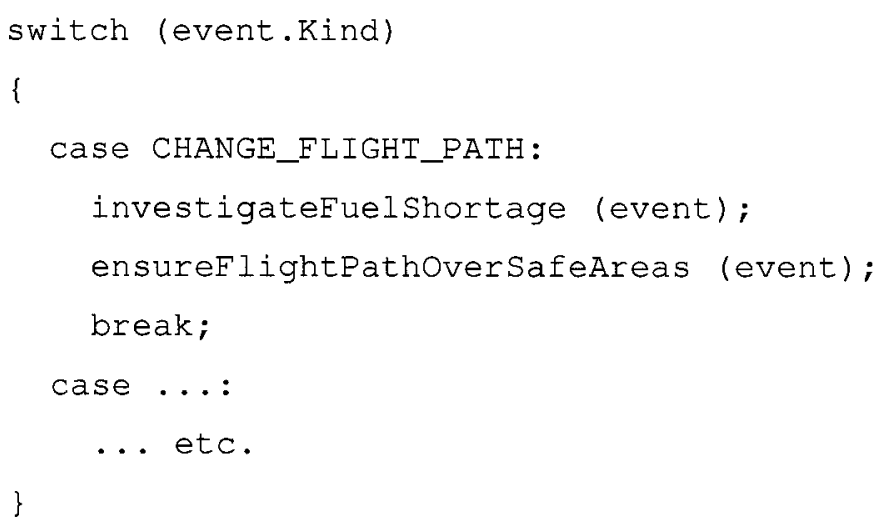

Either way, the Handler class would be invoked as follows:

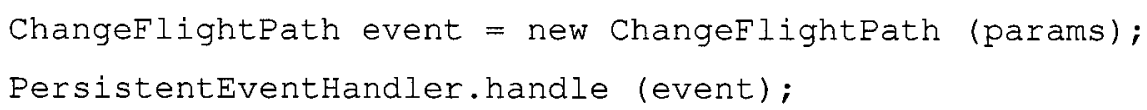

Thus, the PersistentEventHandler static class would know exactly that it should execute the InvestigateFuelShortage (R6) and EnsureflightPathoverSafeAreas (R7) reactions. This is a better approach as it would relief the clients from knowing the reaction specifics. In other words, they can call the handle procedure of the event handler without needing to know what the event is and what its reactions are.

The concepts of events and reactions in this research are related to the UML concepts of signals and operations. A signal is intended to indicate the occurrence of an event of interest. The occurrence of a signal could result in the occurrence of another signal, or in the invocation of an operation. Therefore, either a signal or an operation may be the response to a signal. Thus, signals and operations may be reactions to other signals ("events" in the safety analysis domain). 
In summary, structuring and interpreting events and reaction is an implementation detail rather than a significant design decision. Therefore, it will not be pursued any further in this case study.

\subsection{Design Analysis}

Now that NavigationControllerSubsystem is designed according to the functional and safety requirements, the design model is analysed according to the UML profile's usage scenarios identified in section 2.4. This section will illustrate the usefulness of modeling safety information in the UML model using the proposed UML profile.

\subsubsection{USAGE 1: Provide Safety Requirements}

Safety requirements are provided by the safety and airworthiness engineers. In this case study, the safety requirements resulted from the safety assessment performed in section 7.4, and the safety requirements were listed in section 7.4.5.

Two general requirements were specified in the class diagram for NavigationControllerSubsystem in Figure 15 (see the top of the diagram). One of them was a functional requirement, which indicated that the class diagram must fulfill all FREQ requirements of the subsystem. The second one was a safety requirement, which indicated that the class diagram must fulfill all SREQ requirements of the subsystem. To ensure that every functional and safety requirement is addressed by at least one class, one needs to ensure that every functional and safety requirement is referenced by at least one class using an appropriate stereotype and its tagged values. The $<<$ Rationale $>$ (5.2.14) 
stereotype is the most common for such usage. This is a step towards ensuring that the diagram fulfills those two high-level diagram requirements.

In addition, safety (and functional) requirements were referenced in the UML model using the $<<$ Rationale $>>$ (5.2.14) stereotype. Each model element that implemented at least one safety (or functional) requirement was stereotyped with $<<$ Rationale $>$ (5.2.14). Thus, the design was explicitly and precisely related to the safety requirements. This had several advantages. One particular advantage that is relevant for this usage scenario is that software engineers better consider safety requirements if they have to explicitly relate the design model elements to them. This improves the communication between software and airworthiness/safety engineers because software engineers are now better able to relate the safety requirements to their software designs.

\subsubsection{USAGE 2: Design Safety Requirements in Systems}

Safety requirements were decomposed into events and reactions in section 7.5, which helped design the safety requirements in the software. Event handlers, which perform reactions to events, were designed and stereotyped with $\ll$ Handler $>$ (5.2.19). When events and reactions were modeled, either as a class or as an operation, they were stereotyped with $<<$ Event $>>(5.2 .15)$ and $<<$ Reaction $>>$ (5.2.16), respectively.

As a result of decomposing safety requirements into events and reactions, designing safety requirements in the system reduces to ensuring that safety-related events are detected and that the relevant reactions are properly executed. The events are listed and described in section 7.5.2, and the reactions are listed and described in section 7.5.3. This can be ensured by analysing event handling classes in Figure 15, which were stereotyped 
with $<$ Handler $>$ (5.2.19), and ensuring that the "HandleableEvent" tagged values include all previously identified safety-related events. In addition, it must be ensured that all previously identified safety-related reactions are included in the "PerformedReaction" tagged values, and that all such reactions are properly executed in response to the events that trigger them. This helps ensure that all previously identified safety requirements are accounted for in the design.

All classes that implement safety requirements are stereotyped with $<<$ Rationale $>$ (5.2.14) and their "Reference" tagged values explicitly identify all the requirements that they implement. Therefore, another way to ensure that the safety requirements are accounted for in the design is to analyse the UML model and identify all $<<$ Rationale $>$ (5.2.14) stereotypes. Then, their "Reference" tagged values are analysed to ensure that every safety requirement is referenced by at least one class. A class references a safety requirement if it implements it or at least helps implement it.

One particular use of the $<<$ Rationale $>$ (5.2.14) stereotype and its "Reference" tagged value is to associate reactions to the safety requirements that they implement. This is evident in Figure 14 as each modeled reaction is stereotyped with $<<$ Rationale $>$ (5.2.14) to identify the safety requirements that it implements.

Since this is a highly-safety critical subsystem that has almost as many safety requirements as functional requirements, safety-monitoring is emphasized. Safety monitors are needed to design the safety requirements in the software. Thus, that subsystem has several safety monitors, one for each subsystem on which it depends and one for itself. The monitors can be identified by analysing the model and identifying all 
classes that are stereotyped with $<<$ Monitor $>>$ (5.2.20). Each monitor class explicitly identifies what it monitors through the "MonitoredEntity" tagged value. Therefore, Navigationcontrollersubsystem will be able to track subsystem and class failures and ensure that this does not cause any safety hazards. In fact, once a monitor detects an event of interest, it notifies an appropriate event handler, which is stereotyped with $<<$ Handler>> (5.2.19) itself.

The monitors were designed to detect the safety-critical events of interest to the safety requirements. Once such an event is detected, the appropriate event handler is notified. Once notified, the event handler executes the corresponding reaction for each event. The reactions are intended to alleviate the safety hazards introduced by each event, which is indicated by stereotyping each reaction with $<<$ Reaction $>>$ (5.2.16) and setting its "EffectOnSafetyDirection" tagged value to "Positive". The safety monitoring classes, which are stereotyped with <<Monitor»> (5.2.20) in the design are WingsAndEnginesMonitor, NavigationDatabaseMonitor, NavigationMonitor, and ControllerMonitorAndHandler. The event handlers, stereotyped with $<<$ Handler $>>$ (5.2.19) in the design are ExternalsubsystemsEventHandler and ControllerMonitorAndHandler.

\subsubsection{USAGE 3: Justify Design Decisions}

Justifying design decisions is captured in the "Explanation" tagged value of the $<<$ Rationale > (5.2.14) stereotype. In this case study, a detailed explanation on how the class implements each safety requirement, and often even functional requirement, 
assigned to it was discussed. This explanation was presented in section 7.5.5 and each occurrence was explicitly identified as the value of an "Explanation" tagged value.

\subsubsection{USAGE 4: Monitor Safety}

There are many approaches to monitoring the design and ensuring that it fulfills safety requirements. One way is to ensure that each safety requirement has design elements traceable to it. This case study has used the $<<$ Rationale $>$ (5.2.14) stereotype to trace design elements to safety requirements and provide justifications for this. The following search query can be executed to determine which model elements. Including classes and reactions, are traceable to (e.g. implement) safety requirements:

$$
\text { SEARCH FOR all model elements STEREOTYPED WITH (<<Rationale>>) }
$$

The results can be analysed to determine how safety requirements were designed into the system in USAGE 2, and the "Explanation" tagged value of the $<<$ Rationale $>$ (5.2.14) stereotype can be read to determine the justifications, performed in USAGE 3, for the design decisions performed in USAGE 2.

Executing this search query and then sorting the results per software requirement (i.e. according to the "Reference" tagged value of the $<<$ Rationale $>$ stereotype) tells us which classes and reactions implement each safety requirement. Notice that we do not have events stereotyped with $<<$ Rationale $>$ (5.2.14) because they do not contain executable code that implements safety requirements. The results (classes and reactions) of the above query for each safety requirement are:

SREQ 1 WingsAndEnginesMonitor, ExternalsubsystemsEventHandler, EnableController (R3), DisableController (R4) 


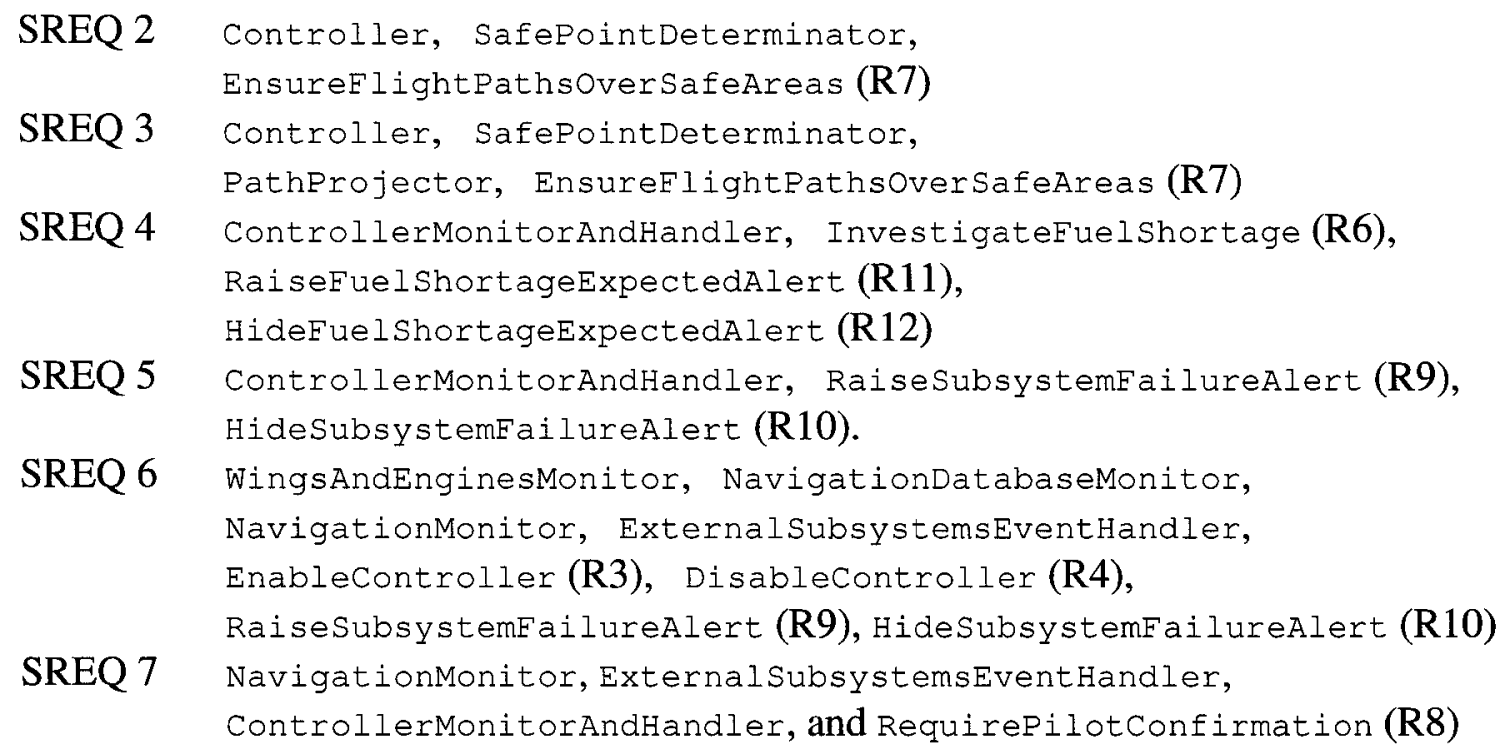

Furthermore, monitoring safety includes ensuring that each event that can have negative effect on safety has one or more appropriate reactions that have a positive effect on safety. This basically means that all hazards caused by events must be treated, and technically removed, by reactions. Thus, each class stereotyped with $<<$ Event $>>(5.2 .15)$ must be referenced at least once by a "ConsequenceOf" tagged value of a $<<$ Reaction $>>$ (5.2.16) stereotype of some class. In this case study, this was presented in sections 7.5 .4 and 7.5.4, specifically in Figure 14 and Table 5, respectively.

Furthermore, one must ensure that all the safety-related events are detectable by monitors. This is ensured through the "DetectableEvent" tagged value of the $<<$ Monitor $\gg>(5.2 .20)$ stereotype. Notice that a monitor can recognize events, but it does not know which reactions may execute in response to each event. In fact, this is the responsibility of an event handler. The following search query can be executed to obtain a list of monitors:

SEARCH FOR all model elements STEREOTYPED WITH (<<Monitor >>) 
Executing this query on the design in Figure 15 gives the following result: ControllerMonitorAndHandler, WingsAndEnginesMonitor, NavigationDatabaseMonitor, and NavigationMonitor.

Then, looking into the "DetectableEvent" tagged value of the $<$ Monitor $>$ (5.2.20) stereotype for each of the classes in the result above tells us which events are detected by the identified monitors. This tells us whether all events of interest are detectable (i.e. the design of monitors is complete) or not.

In addition, events detected by monitors must be handled by event handlers. The "EventHandler" tagged value of the $<<$ Monitor $\gg$ (5.2.20) stereotype for each monitor class identifies the classes that the monitor notifies when any of the events represented by the "DetectableEvent" tagged value occurs.

This case study contained two event handlers, each of which explicitly specifies the events it can handle and the reactions that it performs in response to those events. This information was presented in the "HandleableEvent" and "PerformedReaction" tagged values, respectively, of the $<<$ Handler $>$ (5.2.19) stereotype. A list of event handlers can be obtained by executing the following search query:

SEARCH FOR all model elements STEREOTYPED WITH (<<Handler $>$ )

Executing this search query on the design model in Figure 15 gives the following result: ExternalSubsystemsEventHandler, and ControllerMonitorAndHandler.

Then, one needs to ensure that the "HandleableEvent" and "PerformedReaction" of the $<$ Handler $>$ (5.2.19) stereotype for all handler classes reference all the previously 
determined events and their reactions. This tells us whether all safety-related events of interest are properly handled, through the execution of appropriate reactions, or not.

\subsubsection{USAGE 5: Get Safety Information}

Safety information that is required to prove compliance with airworthiness requirements includes ensuring that each safety requirement is implemented. This was discussed in sections 7.6.1 - 7.6.4.

In addition, safety information that is required by the certification authorities also includes determining hardware/software interfaces as explained in section 6.2.1. This can be obtained by executing the search query described in section 6.2.1. The search query is:

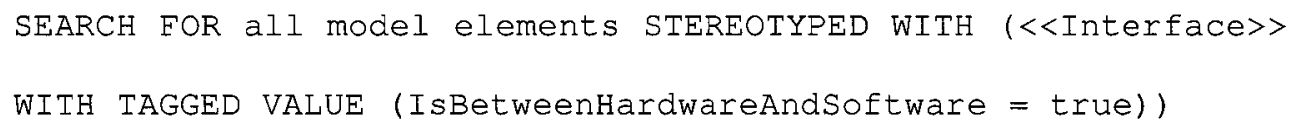

Executing it on NavigationControllersubsystem (section 7.5.5) gives: WingsAndEngines Interface, and NavigationInterface.

Furthermore, the certification authorities require that software levels be specified and submitted as explained in section 6.2.2. This can be obtained by executing the search query in section 6.2.2. This search query is:

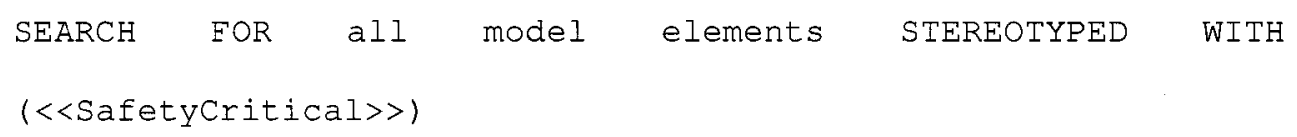

Executing it on the system architecture in section 7.2 gives all the subsystems, namely: 
LEDDisplaysubsystem,

Subsystem, NavigationDatabaseSubsystem, NavigationSubsystem.

The software levels can be obtained from the "CriticalityLevel" tagged value of the $<<$ SafetyCritical $>>(5.2 .17)$ stereotype for each of the above subsystems.

Other information that is required by the certification authorities includes the partitions in the system as explained in section 6.2.4. This can be obtained by executing the search query described in section 6.2.4. The search query is:

SEARCH FOR all model elements STEREOTYPED WITH
(<<Partition>>)

Executing it on the system architecture in section 7.2 gives: NavigationControllerSubsystem.

Furthermore, the airworthiness rules specify that the software level for each subsystem or class be equal to at least that of the highest software level for all subsystems or classes that depend on it $^{2}$. This can be automatically verified in the model by executing the following pseudocode:

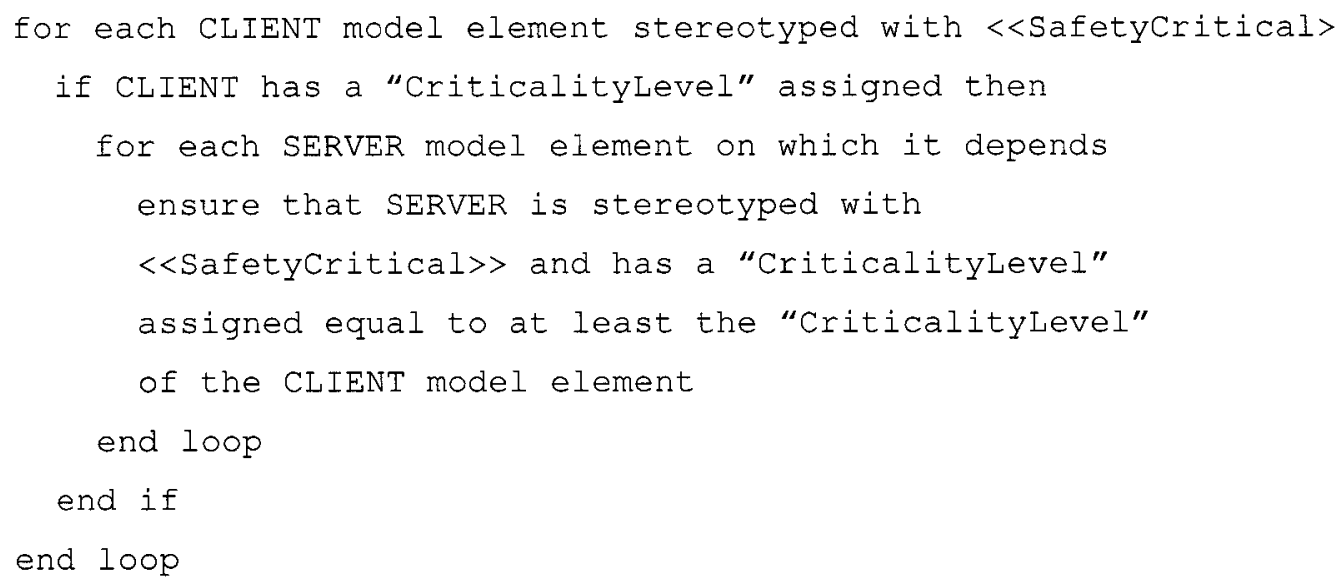


Moreover, the certification authorities require a list and description of all safety monitors.

The monitors and handlers were discussed in sections 7.6.2 and 7.6.4. 


\section{CONCLUSION}

This research has proposed a UML profile for developing software to be compliant with the airworthiness standard, RTCA DO-178B [4]. The profile was based on the safetyrelated concepts that were extracted from the airworthiness standard as well as their refined concepts. As a result, the UML extensions provided by this profile are a consequence of the concepts emphasized by the airworthiness standard.

For example, the airworthiness standard emphasized traceability across requirements, design, and source code. The proposed profile provides specific extensions to model traceability of design elements to requirements, deviation of design elements from original requirements, requirements in the UML model, justification of design and implementation styles according to requirements, and partitioning of the architecture according to the requirements. A detailed analysis on how the UML profile can be used to fulfill each information requirement is presented in section 8.1.

Furthermore, this research has shown how a software model using an appropriate UML profile can be used to effectively generate airworthiness-related information. Such information can be submitted to the certification authorities, or it can be used by the airworthiness engineers to track how software evolves over the lifetime of a project from an airworthiness point of view.

The profile's completeness and usefulness was validated by performing a case study. An aircraft's navigation subsystem was defined and analysed by performing a safety assessment. It was then modeled using the proposed UML profile. Analysing the model 
showed that the UML profile effectively supported the previously identified usage scenarios for safety information. In addition, it is effective in tracing model elements to safety requirements and in automatically generating certification information from a UML model.

To achieve the results presented above, this research had to define the used safety-related concepts precisely. The original safety-related concepts that were extracted from the airworthiness standard were refined into concepts that better grouped related concepts. Furthermore, each safety related concept was described in detail, and attributes were defined to describe the various aspects and dimensions of each concept. A conceptual model (class diagram) was also defined and presented, which precisely defined the relationships across safety-related concepts and modeled them as associations and inheritance relationships. Furthermore, the profile precisely defined the tagged values for each stereotype including its type and multiplicity, and mapped each stereotype and its tagged values to the refined concepts and their attributes and relationships.

It is also important to note that this UML profile builds on top of existing UML model diagrams within a project. In other words, using this UML profile does not require the software engineers to create new diagrams but they must stereotype model elements accordingly. Therefore, the amount of effort involved in using this profile is minimal. This also contribute towards improving communication between safety and airworthiness engineers on the one hand, and software engineers on the other hand. 


\subsection{Fulfilling Requirements}

The proposed UML profile was defined based on the refined safety-related concepts specified in section 3.2. As section 5 illustrates, there exists a stereotype for each of the refined concepts. Therefore, the proposed UML profile is able to model all the refined concepts. Table 6 illustrates how each information requirement is fulfilled by the proposed UML profile.

\begin{tabular}{|c|c|}
\hline IREQ \# & How to Fulfill the Information Requirement \\
\hline IREQ 1 & Use $<<$ SafetyContext $>>$ (5.2.1) stereotype \\
\hline IREQ 2 & Use $<<$ ReliabilityContext $>>(5.2 .2)$ stereotype \\
\hline IREQ 3 & Use $<<$ IntegrityContext $>>(5.2 .3)$ stereotype \\
\hline IREQ 4 & Use $<<$ PerformanceContext $>>(5.2 .4)$ stereotype \\
\hline IREQ 5 & Use $<<$ ConcurrencyContext $>>(5.2 .5)$ stereotype \\
\hline IREQ 6 & Use $<<$ CertificationContext $>>(5.2 .6)$ stereotype \\
\hline IREQ 7 & Use $<<$ ConfigurationContext $>>(5.2 .8)$ stereotype \\
\hline IREQ 8 & Use $<<$ DesignContext $>>(5.2 .7)$ stereotype \\
\hline IREQ 9 & Use $<<$ Requirement $\gg>$ (5.2.9) stereotype and its "Kind" and "Specification" tags \\
\hline IREQ 10 & Use $<<$ Requirement $>>$ (5.2.9) stereotype and its "OfGoal" tag \\
\hline IREQ 11 & Use $<<$ Deviation $>>(5.2 .10)$ stereotype \\
\hline IREQ 12 & Use $<<$ ImplementationStyle $>>$ (5.2.11) stereotype and its "Kind" tag \\
\hline IREQ 13 & $\begin{array}{l}\text { Use }<<\text { BehaviouralStyle }>>(5.2 .12) \text { stereotype with its "Kind" tag equal to "Time- } \\
\text { Related" }\end{array}$ \\
\hline IREQ 14 & $\begin{array}{l}\text { Use }<<\text { BehaviouralStyle }>(5.2 .12) \text { stereotype with its "Kind" tag equal to "State- } \\
\text { Related" }\end{array}$ \\
\hline IREQ 15 & $\begin{array}{l}\text { Use } \ll<\text { Nature }>>(5.2 .13) \text { stereotype and its "Reference" and "Explanation" tags with } \\
\text { its "Kind" tag equal to "COTS" }\end{array}$ \\
\hline IREQ 16 & $\begin{array}{l}\text { Use }<<\text { Nature }>>(5.2 .13) \text { stereotype and its "Reference" and "Explanation" tags with } \\
\text { its "Kind" tag equal to "Previously Developed" }\end{array}$ \\
\hline IREQ 17 & $\begin{array}{l}\text { Use }<<\text { Nature }>>(5.2 .13) \text { stereotype and its "Reference" and "Explanation" tags with } \\
\text { its "Kind" tag equal to "Deactivated" }\end{array}$ \\
\hline IREQ 18 & Use $<<$ Rationale $>$ (5.2.14) stereotype \\
\hline IREQ 19 & Use $<<$ Rationale $>>(5.2 .14)$ stereotype and its "Reference" and "Explanation" tags \\
\hline IREQ 20 & Use $<<$ Event $>>(5.2 .15)$ stereotype \\
\hline IREQ 21 & $\begin{array}{l}\text { Use }<<\text { Event }>>(5.2 .15) \text { stereotype and its "EffectOnSafetyDirection" and } \\
\text { "EffectOnSafetyValue" tags }\end{array}$ \\
\hline
\end{tabular}




\begin{tabular}{|c|c|}
\hline IREQ \# & How to Fulfill the Information Requirement \\
\hline IREQ 22 & Use $<<$ Reaction $>>(5.2 .16)$ stereotype \\
\hline IREQ 23 & Use $<<$ Reaction $>>(5.2 .16)$ stereotype and its "ConsequenceOf" tag \\
\hline IREQ 24 & $\begin{array}{l}\text { Use }<<\text { Reaction }>>(5.2 .16) \text { stereotype and its "EffectOnSafetyDirection" and } \\
\text { "EffectOnSafetyValue" tags }\end{array}$ \\
\hline IREQ 25 & Use $<<$ SafetyCritical $>>(5.2 .17)$ stereotype \\
\hline IREQ 26 & Use $<<$ SafetyCritical $\gg>(5.2 .17)$ stereotype and its "CriticalityLevel" tag \\
\hline IREQ 27 & Use $<<$ Partition $>>(5.2 .18)$ stereotype \\
\hline IREQ 28 & Use $<<$ Handler $>>(5.2 .19)$ stereotype \\
\hline IREQ 29 & Use $<<$ Monitor $>>(5.2 .20)$ stereotype \\
\hline IREQ 30 & Use $<<$ Monitor $>>(5.2 .20)$ stereotype with its "Kind" tag equal to "Safety" \\
\hline IREQ 31 & Use $<<$ Monitor $>>(5.2 .20)$ stereotype with its "Kind" tag equal to "Fault Tolerance" \\
\hline IREQ 32 & Use $<<$ Monitor $>>(5.2 .20)$ stereotype with its "Kind" tag equal to "Integrity" \\
\hline IREQ 33 & Use $<<$ Simulator $\gg>(5.2 .21)$ stereotype \\
\hline IREQ 34 & $\begin{array}{l}\text { Use }<<\text { Simulator }>>(5.2 .21) \text { stereotype and its "SimulatedEntity" and } \\
\text { "SimulationParameter" tags }\end{array}$ \\
\hline IREQ 35 & Use $<<$ Strategy $>>$ (5.2.22) stereotype with its "Kind" tag equal to "Safety" \\
\hline IREQ 36 & Use $<<$ Strategy $>>$ (5.2.22) stereotype with "Kind" tag equal to "Scheduling" \\
\hline IREQ 37 & Use $<<$ Formalism $>>$ (5.2.23) stereotype \\
\hline IREQ 38 & Use $<<$ Complexity $>>$ (5.2.24) stereotype and its "Measure" and "Value" tags \\
\hline IREQ 39 & $\begin{array}{l}\text { Use }<<\text { Interface }>>(5.2 .25) \text { stereotype with its "IsBetweenHardwareAndSoftware" } \\
\text { tag equal to "true" }\end{array}$ \\
\hline IREQ 40 & $\begin{array}{lllll}\text { Use } \quad \text { <Interface > } & (5.2 .25) & \text { stereotype and its } & \text { "ProtocolID", } \\
\text { "InputFunctionParameter", and "OutputFunctionParameter" tags } & \\
\end{array}$ \\
\hline IREQ 41 & Use $<<$ Concurrent $>>(5.2 .26)$ stereotype with its "Role" tag equal to "Active" \\
\hline IREQ 42 & Use $<<$ Concurrent $>>(5.2 .26)$ stereotype with its "Role" tag equal to "Passive" \\
\hline IREQ 43 & Use $<<$ Concurrent $>>(5.2 .26)$ stereotype with its "Role" tag equal to "Resource" \\
\hline IREQ 44 & $\begin{array}{l}\text { Use }<<\text { Concurrent }>>(5.2 .26) \text { stereotype and its "IsShared" tag with its "Role" tag } \\
\text { equal to "Resource" }\end{array}$ \\
\hline IREQ 45 & Use $<<$ Defensive $>>$ (5.2.27) stereotype \\
\hline IREQ 46 & Use $<<$ Defensive $>>(5.2 .27)$ stereotype and its "DefendableInput" tag \\
\hline IREQ 47 & Use $<<$ Configurable $>>(5.2 .28)$ stereotype \\
\hline IREQ 48 & Use $<<$ Configurable $>>(5.2 .28)$ stereotype and its "Kind" tag \\
\hline IREQ 49 & Use $<<$ Configurable $>>(5.2 .28)$ stereotype and its "When" tag \\
\hline IREQ 50 & Use $<<$ Loadable $>>$ (5.2.29) stereotype \\
\hline IREQ 51 & Use $<<$ Configurator $>>(5.2 .30)$ stereotype \\
\hline IREQ 52 & Use $<<$ Replicated $>>$ (5.2.31) stereotype \\
\hline IREQ 53 & Use $<<$ Comparator $>>(5.2 .32)$ stereotype \\
\hline
\end{tabular}




\begin{tabular}{|c|l|}
\hline IREQ \# & \multicolumn{1}{|c|}{ How to Fulfill the Information Requirement } \\
\hline IREQ 54 & Use $<<$ Comparator $>(5.2 .32)$ stereotype and its "PolicyParameter" tag \\
\hline Total & All 54 information requirements are fulfilled \\
\hline
\end{tabular}

Table 6: Using the proposed UML profile to fulfill the information requirements.

\subsection{Open Issues and Future Work}

This research has defined a software safety UML profile and demonstrated how it can help solve the identified challenges. Numerous examples of its usage have been presented (section 5.3). Furthermore, the profile has been applied in a case study (section 7) involving an aircraft navigation controller system - a key software element in every aircraft. Future work could include applying the profile for other systems in diverse organizations, and then soliciting the engineers participating in those projects to identify the strengths and weaknesses of this profile. Such solicitations can be used to generate qualitative and quantitative results in an approach similar to the one used in [46].

This research has focused on modeling safety information in class diagrams. It demonstrated how the proposed UML profile's stereotypes and tagged values can be used to model information in class diagrams. There was little discussion of other types of diagrams, such as dynamic diagrams including object diagrams and statecharts. This has been left for future work. However, the proposed UML profile should be easily transferable for dynamic diagrams.

The proposed UML profile lists the UML meta classes on which each stereotype may be applied (section 5.1). This list may not necessarily be sufficient for all usages. More specifically, certain applications of this profile may determine that it is useful to apply 
certain stereotypes on UML meta classes that are not listed here. Nevertheless, this will form the path in which this profile can evolve in the future.

The refined safety-related concepts (section 3.2), which formed the basis of the UML profile, are mostly based on the general safety-related concepts identified in the airworthiness standard [4]. A standard is normally written in a high-level language as to not restrict the developers following it. Different projects have different airworthiness requirements and/or technical solutions to airworthiness requirements, and hence may have additional refined concepts. This is because UML models can be used as an interface between safety engineers and software developers. This may introduce the need for additional profile stereotypes and tagged values.

Although airworthiness is a subset of safety, it is specific to the aerospace industry. Many other industries exist where safety-critical software is used such as the medical, nuclear, transport, and defence industries. This research was based on the airworthiness standard, and therefore is intended to meet requirements of the aerospace industry. Those requirements may or may not be sufficient in other industries that use different safetyrelated standards. However, an attempt has been made in this research to generalize results as much as possible without compromising the ability to model specific airworthiness concepts and needs. Therefore, this UML profile should be easily tailorable and applicable to other industries.

It was initially thought that it would be best to propose a UML profile that would be as compliant as possible with existing OMG UML profiles. However, it was found that it was better to define concepts for which similar stereotypes existed in other profiles (e.g. 
$<<$ Requirement $\gg>(5.2 .9)$ stereotype is similar to the $<<$ QoSConstrain $\gg>$ and its subtypes in the QoS and FT OMG UML profile [5]). This redefinition allowed this profile to be simple, clear, and most importantly self-contained and independent of other existing profiles. This is important from a maintainability point of view because we may not necessarily need to modify this profile if other OMG UML profiles are revised and newer versions are available.

While some of its stereotypes and tagged values can be used to model safety-related information for systems, this UML profile has focused on modeling them for software. This is jusitified by the fact that the airworthiness standard [4] focuses on software itself. Nevertheless, it may be useful to model such information for systems in future work. In that regard, the OMG System Modeling Language [47], which resuses and extends a subset of UML [30] to model systems, may be helpful. In fact, it may be merged with UML to constitute the base modeling language for a new systems and software UML profile.

Certain extension mechanisms that describe the code were added. Examples of these include using $<<$ ImplementationStyle $>>(5.2 .11)$ to identify recursive code, or code that dynamically allocates memory. Another example is using the $<<$ Complexity $>>(5.2 .24)$ stereotype to identify constraints on the level of code nesting. While they were provided here for completeness and their need in software certification, some of them are generally better addressed by a software code analysis tool that could parse software code, analyse it, and extract this information. The later approach would provide more complete and accurate results, and it would relieve the developers from maintaining this information in the model. Therefore, it may be more appropriate to use such stereotypes and tagged 
values as placeholders for data entered by software analysis tools. This UML profile does not specify the source of the information entered by the software engineers. Thus, a possible future extension could explicitly address this distinction and potentially provide two different values for each piece of information - a specified value, which is entered by the engineers to indicate requirements, and an analysis value, which is entered by a tool to indicate a predicted or measured value. Then, a tool can compare those two sets of values to detect violations.

Most enumeration tagged values for some stereotypes were left open for extension by the users of this profile. This recognizes the fact that each software application may have specific needs or usages whose level of detail that is not addressed here. Therefore, this gives flexibility in a seemingly open UML profile. However, this UML profile lists sample values for each enumeration type that are deemed most useful. Thus, users of this UML profile do not need to do extra work in defining the enumeration values unless they truly need to.

Search queries on UML models were used to illustrate how certification-relevant information can be extracted from a UML model. Those search queries were specified in a Structured Query Language (SQL)-like textual language. This could be refined and eventually lead into the development of an SQL-variant language that is used specifically to search and query UML models. Such a language should be defined such as it is independent of the UML profiles used.

Alternatively, the integration of EMF and OCL seems to be a promising integration of technologies to query UML models. The current state of this technology does not support 
querying UML models for model elements according to criteria specifying the stereotypes and tagged values applied on the model elements. However, EMF and OCL should be easily extensible to support this because it already supports some form of querying UML models and class objects. Such an extension would be supportive of this UML profile as it will allow developers to dynamically query this information. 


\section{SUMMARY}

This research has investigated the relationship between UML and software safety. The airworthiness standard [4] is widely considered as the de-facto safety standard in the aerospace industry. Therefore, modeling software that has to be developed in an environment satisfying the airworthiness standard was considered. Since UML has become the de-facto software modeling language, it was fitting to define a UML profile for modeling safety-critical software. Therefore, even high-level requirements were identified for a UML profile to be able to effectively model safety-critical software developed under the airworthiness standard.

The airworthiness standard was analysed to determine safety-related concepts of interest. A list of 65 safety-related concepts was formed, and the concepts were categorized in eight different but related categories: safety, reliability, integrity, performance, concurrency, certification, design, and configuration.

Given the language difference between standards and UML modeling techniques, the 65 safety-related concepts were refined into 27 concepts that were more appropriate from a software modeling perspective. This refined list of concepts removed duplication across similar concepts, and it defined additional concepts that were not covered by the original 65. The 27 refined safety-related concepts were explained in detail and their inter-concept relationships were formalized through a conceptual model. This allowed us to define 54 information requirements for a candidate UML profile to model those concepts. Those 54 information requirements were traced back to the original 7 high-level concepts. 
After analyzing several existing UML profiles and concluding that they did not fulfill an acceptable percentage of the 54 information requirements, a UML profile was proposed. The profile, composed of 32 stereotypes and their tagged values, was presented in detail. Several examples of using the profile were presented and explained in detail. The profile fulfilled the 54 information requirements and guidance was presented on how it can be used to fulfill each one of the information requirements.

One specific usage of the profile was the ability to automatically provide airworthiness and certification information from a UML model. Therefore, examples of such usage were presented. Each example identified a specific need from the airworthiness standard, and then it presented search queries that a UML modeling tool can execute on a model employing the proposed profile to automatically generate the required information. This is handy for submitting software-related information to the certification authority as well as continuous project monitoring and control by managers and airworthiness engineers that are likely to be less experienced with software.

The UML profile was validated by using it to design and analyse an aircraft's navigation controller subsystem - a key element in every aircraft. The overall system's architecture was presented and explained, and then the navigation controller's subsystem's functional requirements were defined. Then, a safety assessment using the AEA, FMEA, HAZOP, and IA methods was performed, which identified 11 safety hazards relevant to the subsystem under study. Those resulted in 7 safety requirements for the subsystem's software. The subsystem was then designed using the UML profile, and an analysis of the model was performed. The analysis showed that the resultant UML model contained 
information on how the model elements were traceable to the safety requirements, as well as additional information relevant to the certification authorities.

Future work in this area could include using the profile in real-life projects and soliciting participating stakeholders for improvement information. 


\section{REFERENCES}

[1] Nancy G. Leveson, Safeware - System Safety and Computers, Addison-Wesley, 1995

[2] Tom Pender, UML Bible, Wiley, 2003

[3] A. Kleppe, J. Warmer and W. Bast, MDA Explained: The Model Driven Architecture

- Practice and Promise, Addison-Wesley, First Edition, 2003

[4] Radio Technical Commission for Aeronautics (RTCA) Inc., DO-178B - Software Considerations in Airborne Systems and Equipment Certification, December 1992

[5] Object Management Group (OMG) Inc., UML Profile for Modeling Quality of Service and Fault Tolerance Characteristics and Mechanisms, http://www.omg.org/docs/ptc/0505-02.pdf, May 2005

[6] Object Management Group (OMG) Inc., UML Profile for Schedulability, Performance, and Time Specification, http://www.omg.org/docs/formal/05-01-02.pdf, January 2005

[7] Hassan Gomaa, Designing Concurrent, Distributed, and Real-Time Applications with $U M L$, Addison-Wesley, 2000

[8] RTC Magazine, Certification Requirements for Safety-Critical Software, http://www.rtcmagazine.com/home/article.php?id=100010, June 2004

[9] Debra S. Hermann, Software Safety and Reliability, IEEE Computer Society, 1999 
[10] Kelly J. Hayhurst and C. Michael Holloway, Challenges in Software Aspects of Aerospace Systems, NASA Langley Research Center, 2001

[11] US Department of Defense, MIL-STD-498 - Software Development and Documentation, 1994

[12] Institute of Electrical and Electronics Engineers/Electronics Industry Association (IEEE/EIA), IEEE/EIA 12207 - Standard for Software Lifecycle Processing, March 1998

[13] Merriam-Webster Inc., Merriam-Webster's Collegiate Dictionary, Tenth Edition, 1993

[14] Nancy G. Leveson, Stephen S. Cha, John C. Knight, Timothy Shimeall, The Use of Self Checks and Voting in Software Error Detection: An Empirical Study, July 1985

[15] Barry W. Johnson, Design and Analysis of Fault-Tolerant Digital Systems, AddisonWesley, Reading, Massachusetts, 1989

[16] Institute of Electrical and Electronics Engineers (IEEE), IEEE Standard Glossary of Software Engineering Terminology 610.12-1990, Volume 1: Customer and Terminology Standards, IEEE Press, 1999

[17] B. Edmonds, Complexity and Scientific Modelling, Proceedings of the $20^{\text {th }}$ International Wittgenstein Symposium, Austria, August 1997

[18] John J. Marcinak, Encyclopedia of Software Engineering, Wiley-Interscience, 1994

[19] W. P. Stevens, G. J. Myers, L. L. Constantine, Structural Designs, IBM System Journal 13 (2) 115-139, 1974 
[20] R. W. Jensen, C. C. Tonies, Software Engineering, Prentice Hall, 1979

[21] G. J. Myers, Software Reliability Principles and Practices, John Wiley \& Sons, 1976

[22] Bertrand Meyer, Object-Oriented Software Construction, Prentice Hall, Second Edition, 1997

[23] J. B. Wordsworth, The Best from Formal Methods, Information and Software Technology 41(14), pp. 1027-1032, November 1999

[24] International Electrotechnical Commission (IEC), IEC 61508 - Functional Safety of Electrical/Electronic/Programmable Electronic Safety-Related Systems, December 1998

[25] US Department of Defense, MIL-STD-1553B - Aircraft Internal Time-Division Command/Response Multiplex Data Bus, 1986

[26] Jean-Noël Meunier, Frank Lippert, and Ravi Jadhav, RT Modeling with UML for Safety Critical Applications - the HIDOORS Project Example, 2003

[27] Jan Jürjens, Developing Safety-Critical Systems with UML, 2003

[28] Kai T. Hansen and Ingolf Gullesen, Utilizing UML and Patterns for Safety Critical Systems, 2002

[29] Object Management Group (OMG) Inc., Unified Modeling Language: Infrastructure, http://www.omg.org/docs/formal/05-07-05.pdf, March 2006

[30] Object Management Group (OMG) Inc., Unified Modeling Language: Superstructure, http://www.omg.org/docs/formal/05-07-04.pdf, August 2005 
ARTiSAN Software Inc., ARTiSAN Studio,

http://www.artisansw.com/pdf/product_sheets/studio.pdf, September 2006

[32] International Business Machines (IBM) Software, Rational Software Architect, http://www-306.ibm.com/software/awdtools/architect/swarchitect/, August 2006

[33] Telelogic, Rhapsody, http://www.ilogix.com/sublevel.aspx?id=53, October 2006

[34] Frank Budinsky, David Steinberg, Ed Merks, Raymond Ellersick, Timothy J. Grose, Eclipse Modeling Framework: A Developer's Guide, Addison-Wesley, August 2003

[35] Object Management Group (OMG) Inc., Object Constraint Language, http://www.omg.org/docs/formal/06-05-01.pdf, May 2006

[36] Object Management Group (OMG) Inc., MOF 2.0/XMI Mapping Specification v2.1, http://www.omg.org/docs/formal/05-09-01.pdf, September 2005

[37] World Wide Web Consortium, The Extensible Stylesheet Language Family (XSL), http://www.w3.org/Style/XSL/, September 2005

[38] Daniel P. Friedman, Mitchell Wand, Christopher T. Haynes, Essentials of Programming Languages, Second Edition, The MIT Press, January 2001

[39] International Organization for Standardization/ International Electrotechnical Commission, ISO/IEC 14977:1996 - Information Technology - Syntactic Metalanguage - Extended BNF, First Edition, 1996 
[40] International Organization for Standardization/ International Electrotechnical Commission, ISO/IEC 8652:1995 - Information Technology - Programming Languages $-A d a, 1995$

[41] E. D. Lazowska, J. Zahorjan, G. S. Graham, and K. Sevcik, Quantitative System Performance, Prentice Hall, 1984

[42] Juoko Suokas, The Role of Safety Analysis in Accident Prevention, 1988

[43] D. H. Stamatis, Failure Mode and Effect Analysis: FMEA From Theory to Execution, Second Edition, 2003

[44] Trevor Kletz, Lessons from Disaster, Gulf Publishing Company, Houston, 1993

[45] William B. Noble, Developing safe software for critical airborne applications, IEEE $6^{\text {th }}$ Digital Conference, Baltimore, December 1984

[46] Bente Anda, Kai Hansen, Ingolf Gullesen, and Hanne Kristin Thorsen, Experiences from Introducing UML-based Development in a Large Safety-Critical Project, 2004

[47] Object Management Group (OMG) Inc., Systems Modeling Language, http://www.omg.org/cgi-bin/apps/doc?ptc/06-05-04.pdf, May 2006 


\section{Appendix A Examples of Safety/Risk Assessment Methods}

\begin{tabular}{|c|c|c|c|}
\hline Method & Analyzed Subjects & Output & Comments \\
\hline $\begin{array}{l}\text { Action Error } \\
\text { Analysis (AEA) }\end{array}$ & $\begin{array}{l}\text { Human-machine } \\
\text { interactions }\end{array}$ & $\begin{array}{l}\text { Consequences of } \\
\text { actions that operators } \\
\text { perform at the wrong } \\
\text { time, or do not perform } \\
\text { when they should }\end{array}$ & $\begin{array}{l}\text { Similar to FMEA but is } \\
\text { applied to steps in human } \\
\text { procedures rather than } \\
\text { hardware or components }\end{array}$ \\
\hline $\begin{array}{l}\text { Cause- } \\
\text { Consequence } \\
\text { Analysis (CCA) }\end{array}$ & Critical events & $\begin{array}{l}\text { Causes and } \\
\text { consequences of critical } \\
\text { events }\end{array}$ & $\begin{array}{l}\text { Unlike fault trees, it } \\
\text { explicitly shows the } \\
\text { sequence of events. } \\
\text { Unlike event trees, it } \\
\text { allows the representation } \\
\text { of time delays, alternative } \\
\text { consequence paths, and } \\
\text { combination of events }\end{array}$ \\
\hline $\begin{array}{l}\text { Event Tree } \\
\text { Analysis (ETA) }\end{array}$ & Critical events & $\begin{array}{l}\text { Consequences of } \\
\text { critical events }\end{array}$ & $\begin{array}{l}\text { Is a version of FTA that } \\
\text { is tailored to large and } \\
\text { complex systems. Breaks } \\
\text { large problems into } \\
\text { smaller ones to which } \\
\text { FTA may be applied }\end{array}$ \\
\hline $\begin{array}{l}\text { Failure Modes and } \\
\text { Effects Analysis } \\
\text { (FMEA) }\end{array}$ & Possible failures & $\begin{array}{l}\text { Probabilities of failures. } \\
\text { Overall probability that } \\
\text { the product will operate } \\
\text { without a failure for a } \\
\text { specific period of time }\end{array}$ & $\begin{array}{l}\text { Reliability-oriented rather } \\
\text { than safety-oriented. } \\
\text { Emphasizes correct } \\
\text { functioning rather than } \\
\text { hazards and risks. } \\
\text { Concentrates on single } \\
\text { events of failures }\end{array}$ \\
\hline
\end{tabular}




\begin{tabular}{|c|c|c|c|}
\hline Method & Analyzed Subjects & Output & Comments \\
\hline $\begin{array}{l}\text { Failure Modes, } \\
\text { Effects, and } \\
\text { Criticality } \\
\text { Analysis } \\
\text { (FMECA) }\end{array}$ & Possible failures & $\begin{array}{l}\text { Same as FMEA but it } \\
\text { includes the criticalities } \\
\text { of failures }\end{array}$ & $\begin{array}{l}\text { Is an extended FMEA } \\
\text { that examines the } \\
\text { criticality of each event in } \\
\text { more detail. Concentrates } \\
\text { on single events of } \\
\text { failures }\end{array}$ \\
\hline $\begin{array}{l}\text { Fault Hazard } \\
\text { Analysis (FHA) }\end{array}$ & $\begin{array}{l}\text { Possible failures } \\
\text { that may result in } \\
\text { accidents }\end{array}$ & $\begin{array}{l}\text { Similar to FMEA or } \\
\text { FMECA, but it } \\
\text { considers a different } \\
\text { scope }\end{array}$ & $\begin{array}{l}\text { Causes of failures are } \\
\text { considered over a wide } \\
\text { scope that even includes } \\
\text { human errors, procedural } \\
\text { deficiencies, and } \\
\text { environmental conditions. } \\
\text { Concentrates on single } \\
\text { events of failures that } \\
\text { may cause accidents }\end{array}$ \\
\hline $\begin{array}{l}\text { Fault Tree } \\
\text { Analysis (FTA) }\end{array}$ & $\begin{array}{l}\text { Previously- } \\
\text { identified hazards }\end{array}$ & $\begin{array}{l}\text { Causes of the } \\
\text { previously-identified } \\
\text { hazards, fault trees and } \\
\text { Boolean expressions } \\
\text { for them }\end{array}$ & $\begin{array}{l}\text { Hazards should have } \\
\text { already been identified by } \\
\text { other methods. A popular } \\
\text { method but not scalable } \\
\text { to large and complex } \\
\text { systems. Considers } \\
\text { relationships across } \\
\text { events that cause hazards }\end{array}$ \\
\hline $\begin{array}{l}\text { Hazards and } \\
\text { Operability } \\
\text { Analysis } \\
\text { (HAZOP) }\end{array}$ & $\begin{array}{l}\text { System design and } \\
\text { operating intentions }\end{array}$ & $\begin{array}{l}\text { Possible deviations } \\
\text { from the design and } \\
\text { operating intentions, } \\
\text { and hazards that result } \\
\text { from them }\end{array}$ & $\begin{array}{l}\text { Uses a qualitative } \\
\text { approach. Labour } \\
\text { intensive. Does not } \\
\text { require that the hazards } \\
\text { be previously identified }\end{array}$ \\
\hline $\begin{array}{l}\text { Interface Analyses } \\
\text { (IA) }\end{array}$ & $\begin{array}{l}\text { Inter-component } \\
\text { interfaces }\end{array}$ & $\begin{array}{l}\text { Connection failures that } \\
\text { can lead to failure } \\
\text { propagations }\end{array}$ & $\begin{array}{l}\text { Similar to HAZOP but is } \\
\text { more general }\end{array}$ \\
\hline
\end{tabular}




\begin{tabular}{|c|c|c|c|}
\hline Method & Analyzed Subjects & Output & Comments \\
\hline $\begin{array}{l}\text { Management } \\
\text { Oversight and } \\
\text { Risk Tree Analysis } \\
\text { (MORT) }\end{array}$ & $\begin{array}{l}\text { Managerial } \\
\text { functions, human } \\
\text { behaviour, and } \\
\text { environmental } \\
\text { factors }\end{array}$ & $\begin{array}{l}\text { Problems, defects, and } \\
\text { oversights that create } \\
\text { hazards or prevent their } \\
\text { early identification by } \\
\text { poor planning, } \\
\text { inadequate operational } \\
\text { checks, or limited } \\
\text { information-exchange } \\
\text { within the organization. }\end{array}$ & Checklist-based \\
\hline $\begin{array}{ll}\text { State } & \text { Machine } \\
\text { Hazard } & \text { Analysis } \\
\text { (SMHA) } & \end{array}$ & $\begin{array}{l}\text { Hazardous states in } \\
\text { software state } \\
\text { machines }\end{array}$ & $\begin{array}{l}\text { Conditions that cause } \\
\text { the software to enter } \\
\text { the hazardous states }\end{array}$ & $\begin{array}{l}\text { Intended for software. } \\
\text { Works on the model } \\
\text { rather than the design } \\
\text { itself }\end{array}$ \\
\hline
\end{tabular}

Table 7: Examples of safety or risk assessment methods. 


\section{Appendix B Examples of Safety-Related Standards}

\begin{tabular}{|c|c|c|}
\hline Industry & Owner & Standard \\
\hline \multirow[t]{5}{*}{ Aerospace } & $\begin{array}{l}\text { Radio Technical } \\
\text { Commission for } \\
\text { Aeronautics (RTCA) }\end{array}$ & $\begin{array}{l}\text { DO-178B, Software Considerations in Airborne } \\
\text { Systems and Equipment Certification }\end{array}$ \\
\hline & $\begin{array}{l}\text { European Space Agency } \\
\text { (ESA) }\end{array}$ & $\begin{array}{l}\text { Set of Several Standards - ECSS-Q-00A, ECSS-Q- } \\
\text { 20A, ECSS-Q-30A, ECSS-Q-40A, ECSS-Q-80A, } \\
\text { ECSS-Q-80-2, ECSS-Q-80-3, ECSS-Q-80-4 }\end{array}$ \\
\hline & $\begin{array}{lr}\text { National } & \text { Aeronautics and } \\
\text { Space } & \text { Administration } \\
\text { (NASA) } & \end{array}$ & $\begin{array}{l}\text { NASA-STD-8719.13A }- \text { Software Safety, } \\
\text { September } 1997\end{array}$ \\
\hline & $\begin{array}{lr}\text { National } & \text { Aeronautics and } \\
\text { Space } & \text { Administration } \\
\text { (NASA) } & \end{array}$ & $\begin{array}{l}\text { NASA-GB-1740.13-96 - NASA Guidebook for } \\
\text { Safety Critical Software - Analysis and } \\
\text { Development, September } 1997\end{array}$ \\
\hline & $\begin{array}{l}\text { American Institute of } \\
\text { Aeronautics } \\
\text { Astronautics (AIAA) }\end{array}$ & $\begin{array}{l}\text { R-013-1992 - Recommended Practice: Software } \\
\text { Reliability, } 1992\end{array}$ \\
\hline Biomedical & $\begin{array}{l}\text { International } \\
\text { Electrotechnical } \\
\text { Commission (IEC) }\end{array}$ & $\begin{array}{l}\text { 601-1-4(1996-06) - Medical Electrical Equipment } \\
\text { - Part 1: General Requirements for Safety - } 4 . \\
\text { Collateral Standard: Programmable Electric } \\
\text { Medical Systems, June } 1996\end{array}$ \\
\hline \multirow[t]{3}{*}{ Defence } & $\begin{array}{l}\text { U.S. Department of } \\
\text { Defense (DoD) }\end{array}$ & $\begin{array}{l}\text { MIL-STD-882D - Standard Practice for System } \\
\text { Safety, February } 2000\end{array}$ \\
\hline & $\begin{array}{l}\text { U.K. Ministry of Defence } \\
(\mathrm{MoD})\end{array}$ & $\begin{array}{l}\text { DEF STAN 00-55 - Requirements for Safety } \\
\text { Related Software in Defence Equipment, August } \\
1997\end{array}$ \\
\hline & $\begin{array}{l}\text { North Atlantic Treaty } \\
\text { Organization (NATO) }\end{array}$ & $\begin{array}{l}\text { Commercial Off-the-Shelf (COTS) Software } \\
\text { Acquisition Guidelines and COTS Policy Issues, } \\
\text { January } 1996\end{array}$ \\
\hline
\end{tabular}




\begin{tabular}{|c|c|c|}
\hline Industry & Owner & Standard \\
\hline \multirow[t]{2}{*}{ Nuclear Power } & $\begin{array}{l}\text { International } \\
\text { Electrotechnical } \\
\text { Commission (IEC) }\end{array}$ & $\begin{array}{l}\text { 61508:1986-09-Software for Computers in Safety } \\
\text { Systems of Nuclear Power Stations, including the } \\
\text { First Supplement, 60880-1 (FDIS), } 1977\end{array}$ \\
\hline & $\begin{array}{l}\text { Ontario Hydro Nuclear and } \\
\text { Atomic Energy Canada, } \\
\text { Ltd. (AECL) }\end{array}$ & $\begin{array}{l}\text { CE-1001-STD }- \text { Standard for Software } \\
\text { Engineering of Safety Critical Software, January } \\
1995\end{array}$ \\
\hline \multirow[t]{3}{*}{ Transportation } & $\begin{array}{l}\text { European Committee for } \\
\text { Electrotechnical } \\
\text { Standardisation } \\
\text { (CENELEC) }\end{array}$ & $\begin{array}{l}\text { EN 50128:2001 - Railway Applications: Software } \\
\text { for Railway Control and Protection Systems }\end{array}$ \\
\hline & $\begin{array}{l}\text { Motor Industry Software } \\
\text { Reliability Association } \\
\text { (MISRA) }\end{array}$ & $\begin{array}{l}\text { Development Guidelines for Vehicle-Based } \\
\text { Software, November } 2001\end{array}$ \\
\hline & $\begin{array}{l}\text { Society of Automotive } \\
\text { Engineers (SAE) }\end{array}$ & $\begin{array}{l}\text { JA } 1002 \text { - Software Reliability Program Standard, } \\
\text { July } 2004\end{array}$ \\
\hline \multirow[t]{4}{*}{$\begin{array}{l}\text { Non-Industry } \\
\text { Specific }\end{array}$} & $\begin{array}{l}\text { International } \\
\text { Electrotechnical } \\
\text { Commission (IEC) }\end{array}$ & 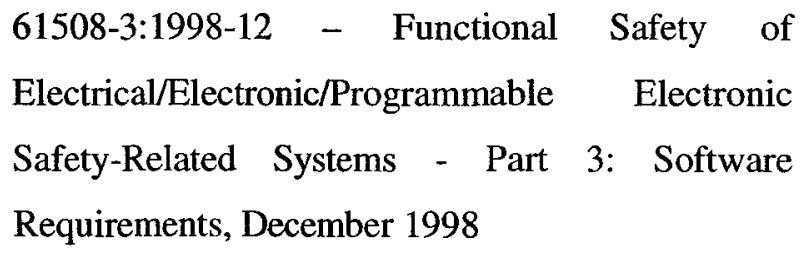 \\
\hline & $\begin{array}{l}\text { International } \\
\text { Electrotechnical } \\
\text { Commission (IEC) }\end{array}$ & $\begin{array}{l}\text { 300-3-9:1995-12 - Dependability Management - } \\
\text { Part 3: Application Guide - Section 9: Risk } \\
\text { Analysis of Technological Systems, December } \\
1995\end{array}$ \\
\hline & $\begin{array}{l}\text { International Organization } \\
\text { for Standardization (ISO) }\end{array}$ & $\begin{array}{l}\text { 15026:1998-04-29 }- \text { System and Software } \\
\text { Integrity Levels, April } 1998\end{array}$ \\
\hline & $\begin{array}{l}\text { Institution of Electrical } \\
\text { Engineers (IEE) }\end{array}$ & $\begin{array}{l}\text { Software Engineering Methods for Safe } \\
\text { Programmable Logic Controllers (SEMSPLC) } \\
\text { Guidelines - Safety-Related Application Software } \\
\text { for Programmable Logic Controllers, September } \\
1996\end{array}$ \\
\hline
\end{tabular}




\begin{tabular}{|l|lr|l|}
\hline Industry & \multicolumn{2}{|c|}{ Owner } & \multicolumn{1}{|c|}{ Standard } \\
\hline & $\begin{array}{l}\text { Institute of Electrical \& } \\
\text { Electronic }\end{array}$ & $\begin{array}{l}\text { Std. 982.1-1989 and 982.2-1989 - Measures to } \\
\text { (IEEE) }\end{array}$ & Engineers \\
Produce Reliable Software
\end{tabular}

Table 8: Some of the many safety-related standards that exist for several industries. 


\title{
Appendix C Concept Identification and Categorization from
}

\author{
the Airworthiness Standard
}

\section{C.1 Primarily Safety Concepts}

\begin{tabular}{|c|c|c|}
\hline Concept & Description & $\begin{array}{l}\text { RTCA DO- } \\
\text { 178B Section }\end{array}$ \\
\hline Failure Condition & $\begin{array}{l}\text { The effect that one or more failures cause on, or } \\
\text { contribute to, the aircraft and its occupants, directly or } \\
\text { indirectly, considering relevant adverse operational and } \\
\text { environmental conditions [4]. }\end{array}$ & 2.2 \\
\hline $\begin{array}{l}\text { Failure Condition } \\
\text { Category }\end{array}$ & $\begin{array}{l}\text { Failure conditions are categorized according to the } \\
\text { severity of their effects as defined in some standard [4]. }\end{array}$ & $\begin{array}{l}2.2 \\
2.2 .1\end{array}$ \\
\hline Level of Confidence & $\begin{array}{l}\text { In the context of software safety, the level of confidence } \\
\text { is the extent of the assurance to which the software is } \\
\text { believed to exhibit the desired behaviour of safety, with } \\
\text { respect to the system in which it is deployed. }\end{array}$ & $\begin{array}{l}4.1 \\
6.4\end{array}$ \\
\hline $\begin{array}{l}\text { Loadable Software } \\
\text { Indicator }\end{array}$ & $\begin{array}{l}\text { A hardware or software that is used to indicate the status } \\
\text { of the field-loadable software (see section C.7). This } \\
\text { indicator should be able to detect incorrect software } \\
\text { and/or hardware and/or aircraft combinations and should } \\
\text { provide protection appropriate to the failure condition of } \\
\text { the function [4]. }\end{array}$ & 2.5 \\
\hline Safeguard & A technical contrivance to prevent accident [13]. & $\begin{array}{l}7.2 .8 \\
11.4\end{array}$ \\
\hline Safety Feature & $\begin{array}{l}\text { A prominent part or characteristic [13], which is } \\
\text { intended to increase the safety of the system. }\end{array}$ & $\begin{array}{l}4.4 \\
11.1\end{array}$ \\
\hline
\end{tabular}




\begin{tabular}{|c|c|c|}
\hline Concept & Description & $\begin{array}{l}\text { RTCA DO- } \\
\text { 178B Section }\end{array}$ \\
\hline Safety Monitoring & $\begin{array}{l}\text { Safety monitoring is a means of protecting against } \\
\text { specific failure conditions by directly monitoring a } \\
\text { function for failures which would contribute to the } \\
\text { failure condition. Monitoring is usually associated with } \\
\text { activities done over an extended period of time where } \\
100 \% \text { witnessing is considered impractical or } \\
\text { unnecessary. Monitoring permits authentication that the } \\
\text { claimed activity was performed as planned [4]. } \\
\text { Examples of safety-monitors include watchdog timers, } \\
\text { reasonableness checks, and cross-channel comparisons } \\
\text { [4]. }\end{array}$ & $\begin{array}{l}2.1 .1 \\
2.3 .2 \\
11.9\end{array}$ \\
\hline Safety Objective & $\begin{array}{l}\text { A safety-related goal, which often results in safety } \\
\text { requirements and safety constraints. }\end{array}$ & 4.1 \\
\hline Safety Requirement & $\begin{array}{l}\text { A safety requirement is a non-functional requirement } \\
\text { whose objective is to increase the level of safety. And } \\
\text { while functional requirements often focus on what the } \\
\text { system shall do, safety-related requirement focus on } \\
\text { both what the system shall and shall not do [1]. }\end{array}$ & $\begin{array}{l}2.1 .1 \\
5.1\end{array}$ \\
\hline Safety Response & $\begin{array}{l}\text { A safety response is an action that a software component } \\
\text { performs as a result of detecting the occurrence of some } \\
\text { safety-related failure condition that it provides immunity } \\
\text { to. }\end{array}$ & 2.1 .1 \\
\hline Safety Strategy & $\begin{array}{l}\text { A strategy is the art of devising or employing plans or } \\
\text { stratagems toward a goal [13]. The goal of a safety } \\
\text { strategy is to increase the safety of a system, which is } \\
\text { often done through design decisions. }\end{array}$ & 2.1 .1 \\
\hline
\end{tabular}




\begin{tabular}{|c|c|c|}
\hline Concept & Description & $\begin{array}{l}\text { RTCA DO- } \\
\text { 178B Section }\end{array}$ \\
\hline Safety-Critical & $\begin{array}{l}\text { A safety-critical system is any system that can directly } \\
\text { or indirectly cause a loss of human life [1]. Examples of } \\
\text { such systems include transportation vehicles, air traffic } \\
\text { controllers, medical devices, nuclear reactors, and } \\
\text { military equipment and vehicles. A loss of human life } \\
\text { may be caused by accidents, which may be the results of } \\
\text { hazards introduced by the system. }\end{array}$ & \\
\hline Software Level & $\begin{array}{l}\text { Assigning different levels to software components is a } \\
\text { means of classifying software components according to } \\
\text { their contribution to potential failure conditions as } \\
\text { determined by the system safety assessment process. } \\
\text { Having different software levels imply different levels } \\
\text { of effort are required to show compliance with different } \\
\text { failure condition categories [4]. }\end{array}$ & $\begin{array}{l}2.2 \\
2.2 .2\end{array}$ \\
\hline Unsafe Action & $\begin{array}{l}\text { An action that can directly or indirectly contribute to the } \\
\text { occurrence of a hazardous system state, which may } \\
\text { result in an accident. Such an action may occur with or } \\
\text { without an explicit action by the software or system } \\
\text { user. }\end{array}$ & \\
\hline
\end{tabular}

Table 9: Safety-related concepts that are classified as "primarily safety". 


\section{C.2 Primarily Reliability Concepts}

\begin{tabular}{|c|c|c|}
\hline Concept & Description & $\begin{array}{l}\text { RTCA DO- } \\
\text { 178B Section }\end{array}$ \\
\hline Comparator (Voter) & $\begin{array}{l}\text { When multiple-version dissimilar software is used, } \\
\text { voting refers to detecting faults caused by some } \\
\text { dissimilar versions of the software. This is done by } \\
\text { assuming that the majority of the dissimilar software } \\
\text { versions produce a correct output [14]. A voter or a } \\
\text { comparator is a software component that analyzes the } \\
\text { results of the multiple software versions and outputs the } \\
\text { result of the voting. }\end{array}$ & 2.3 .2 \\
\hline $\begin{array}{l}\text { Defensive } \\
\text { Programming }\end{array}$ & $\begin{array}{l}\text { Defensive programming is based on the principle that } \\
\text { the programmer makes as few assumptions as } \\
\text { reasonably possible. Extra code is written to check that } \\
\text { the software is in correct state at selected checkpoints, } \\
\text { such as the beginning or end of an operation. This } \\
\text { allows the software to detect incorrect states and react } \\
\text { appropriately to ensure the continued execution of the } \\
\text { system. }\end{array}$ & 4.5 \\
\hline Error Detection & $\begin{array}{l}\text { The process of realizing that an error has occurred [15]. } \\
\text { In a fault-tolerant design, this may be implemented as } \\
\text { part of the software or hardware. }\end{array}$ & 4.2 \\
\hline Exception Handling & $\begin{array}{l}\text { Exception handling is a programming technique of most } \\
\text { modern programming languages to handle situations } \\
\text { where abnormal conditions arise. When an exception } \\
\text { occurs, the software flow of control resumes in an } \\
\text { exception handler, which generally handles selected } \\
\text { exceptions and allows the software execution to } \\
\text { continue. }\end{array}$ & 11.7 \\
\hline
\end{tabular}




\begin{tabular}{|c|c|c|}
\hline Concept & Description & $\begin{array}{l}\text { RTCA DO- } \\
\text { 178B Section }\end{array}$ \\
\hline Failure & $\begin{array}{l}\text { A failure is the inability of a system or system } \\
\text { component to perform a required function within } \\
\text { specified limits. It may be produced when a fault is } \\
\text { encountered [4]. }\end{array}$ & 2.2 \\
\hline Fault & $\begin{array}{l}\text { A fault is a manifestation of an error in software that, if } \\
\text { it occurs, may cause a failure [4], which is a deviation in } \\
\text { the expected performance of the system [15]. }\end{array}$ & 2.1 .1 \\
\hline Fault Containment & $\begin{array}{l}\text { In designing and implementing fault tolerant systems, } \\
\text { fault containment is the process of preventing an error } \\
\text { from propagating within a system. Fault containment is } \\
\text { one of four steps required to reconfigure a system from } \\
\text { a faulty state back to some operational state. The other } \\
\text { three steps are fault detection, fault location, and fault } \\
\text { recovery [15]. }\end{array}$ & 2.1 .2 \\
\hline Fault Detection & $\begin{array}{l}\text { The process of realizing that a fault has occurred [15]. In } \\
\text { a fault-tolerant design, this may be implemented as part } \\
\text { of the software or hardware. }\end{array}$ & $\begin{array}{l}2.1 .1 \\
11.9\end{array}$ \\
\hline Fault Tolerance & $\begin{array}{l}\text { The built-in capability of a system or software to } \\
\text { provide continued correct execution in the presence of a } \\
\text { limited number of hardware or software faults [4]. }\end{array}$ & $\begin{array}{l}2.1 .1 \\
4.4 \\
11.1\end{array}$ \\
\hline Immunity & $\begin{array}{l}\text { The quality or state of being immune [13]. A system or } \\
\text { software is immune to some failure condition if it can } \\
\text { detect it and perform an appropriate safety response that } \\
\text { renders it harmless. }\end{array}$ & 2.1.1 \\
\hline $\begin{array}{l}\text { Multiple-Version } \\
\text { Dissimilar Software }\end{array}$ & $\begin{array}{l}\text { A form of fault tolerance design technique where a set } \\
\text { of two or more programs developed separately to satisfy } \\
\text { the same functional requirements are used. Errors } \\
\text { specific to one of the versions are detected by } \\
\text { comparison of the multiple outputs [4]. }\end{array}$ & $\begin{array}{l}2.1 .1 \\
2.3 .2 \\
11.1 \\
11.3\end{array}$ \\
\hline
\end{tabular}




\begin{tabular}{|l|l|l|}
\hline \multicolumn{1}{|c|}{ Concept } & \multicolumn{1}{|c|}{ Description } & \multicolumn{1}{|c|}{$\begin{array}{c}\text { RTCA DO- } \\
\text { 178B Section }\end{array}$} \\
\hline Software \\
Redundancy & $\begin{array}{l}\text { Redundancy is the use of redundant components, which } \\
\text { is exceeding what is necessary or normal [13]. Software } \\
\text { redundancy implies using multiple-version dissimilar } \\
\end{array}$ & 2.1 .1 \\
& software. & \\
\hline
\end{tabular}

Table 10: Safety-related concepts that are classified as "primarily reliability".

\section{C.3 Primarily Integrity Concepts}

\begin{tabular}{|l|l|l|}
\hline \multicolumn{1}{|c|}{ Concept } & \multicolumn{1}{|c|}{ Description } & \multicolumn{1}{|c|}{$\begin{array}{c}\text { RTCA DO- } \\
\text { 178B Section }\end{array}$} \\
\hline Accuracy & $\begin{array}{l}\text { Degree of conformity of a measure to a standard or a } \\
\text { true value [13]. }\end{array}$ & 11.9 \\
\hline Discontinuity & $\begin{array}{l}\text { A mathematical discontinuity is the property of not } \\
\text { being continuous. A continuous function has the } \\
\text { property that the absolute value of the numerical } \\
\text { difference between the value at a given point and the } \\
\text { value at any point in a neighbourhood of the given point } \\
\text { can be made as close to zero as desired by choosing the } \\
\text { neighbourhood small enough [13]. }\end{array}$ & 6.3 .2 \\
\hline Integrity Check & $\begin{array}{l}\text { The act of testing or verifying the integrity of an object. } \\
\text { Integrity is the quality or state of being complete [13], } \\
\text { accurate, and precise. }\end{array}$ & 11.16 \\
\hline Precision & $\begin{array}{l}\text { The degree of refinement with which an operation is } \\
\text { performed or a measurement stated [13]. }\end{array}$ & 11.9 \\
\hline and commercial-off-the-shelf software [4]. & $\begin{array}{l}\text { Software that provides protection for user modifications } \\
\text { in user-modifiable software, option-selectable software, }\end{array}$ & 2.4 \\
\hline
\end{tabular}

Table 11: Safety-related concepts that are classified as "primarily integrity". 


\section{C.4 Primarily Performance Concepts}

\begin{tabular}{|l|l|l|}
\hline \multicolumn{1}{|c|}{ Concept } & \multicolumn{1}{|c|}{ Description } & \multicolumn{1}{|c|}{$\begin{array}{c}\text { RTCA DO- } \\
\text { 178B Section }\end{array}$} \\
\hline Scheduling Strategy & $\begin{array}{l}\text { A strategy is the art of devising or employing plans or } \\
\text { stratagems toward a goal [13]. The goal of a scheduling } \\
\text { strategy is to determine how various active components } \\
\text { share resources. Examples of scheduling strategies } \\
\text { include round robin, rate monotonic, and earliest } \\
\text { deadline first [6]. }\end{array}$ & 11.1 \\
\hline Time-Related & $\begin{array}{l}\text { Software functionality whose output or behaviour is a } \\
\text { function of time such as filters, integrators, and delays. }\end{array}$ & 6.4 .2 .1 \\
\hline
\end{tabular}

Table 12: Safety-related concepts that are classified as "primarily performance".

\section{C.5 Primarily Concurrency Concepts}

\begin{tabular}{|c|c|c|}
\hline Concept & Description & $\begin{array}{l}\text { RTCA DO- } \\
\text { 178B Section }\end{array}$ \\
\hline Active & $\begin{array}{l}\text { An active component is a component that is capable of } \\
\text { generating stimuli concurrently or pseudo (seemingly) } \\
\text { concurrently without being prompted by an explicit } \\
\text { stimulus instance (i.e., devices that appear capable of } \\
\text { "spontaneous" unprompted behaviour such as hardware, } \\
\text { operating system processes and threads, etc.) [6]. In } \\
\text { terms of a software component, package, or object, } \\
\text { activeness implies that a thread is continually executing } \\
\text { within the context of that software component, package, } \\
\text { or object. }\end{array}$ & 12.3 .3 \\
\hline Multi-Tasking & $\begin{array}{l}\text { Software that runs with more than one flow of control, } \\
\text { i.e. concurrent software. Different flows of control may } \\
\text { interact at which point their interactions need to be } \\
\text { managed. }\end{array}$ & 11.7 \\
\hline
\end{tabular}




\begin{tabular}{|l|l|l|}
\hline \multicolumn{1}{|c|}{ Concept } & \multicolumn{1}{|c|}{ Description } & \multicolumn{1}{|c|}{$\begin{array}{c}\text { RTCA DO- } \\
\text { 178B Section }\end{array}$} \\
\hline Passive & $\begin{array}{l}\text { A passive component is a component that cannot } \\
\text { generate its own behaviour, but only reacts when } \\
\text { prompted by a stimulus [6]. In terms of a software } \\
\text { component, package, or object, passiveness implies that } \\
\text { there is no thread that is continually executing within the } \\
\text { context of that software component, package, or object, } \\
\text { but its code is executed as a result of some other active } \\
\text { software component, package, or object. A passive } \\
\text { element is generally event-driven. }\end{array}$ & 12.3 .3 \\
\hline Shared Resource & $\begin{array}{l}\text { A resource that is shared across multiple software } \\
\text { modules or flows of control (software threads or } \\
\text { processes). Examples of resources include memory, } \\
\text { CPU cycles, network, and others. }\end{array}$ & 11.1 \\
\hline
\end{tabular}

Table 13: Safety-related concepts that are classified as "primarily concurrency". 


\section{C.6 Primarily Certification Concepts}

\begin{tabular}{|c|c|c|}
\hline Concept & Description & $\begin{array}{c}\text { RTCA DO- } \\
\text { 178B Section }\end{array}$ \\
\hline Certification & $\begin{array}{l}\text { Certification is the legal recognition by the certification } \\
\text { authority that a product, service, organization or person } \\
\text { complies with some requirements. Such certification } \\
\text { comprises the activity of technically checking the } \\
\text { product, service, organization or person and the formal } \\
\text { recognition of compliance with the applicable } \\
\text { requirements by issue of a certificate, license, approval } \\
\text { or other documents as required by national laws and } \\
\text { procedures. In particular, certification of a produt } \\
\text { involves: (a) the process of assessing the design of the } \\
\text { product to ensure that it complies with a set of standards } \\
\text { to that type of product so as to demonstrate an } \\
\text { acceptable level of safety; (b) the process of assessing } \\
\text { the product to ensure that it conforms with the certified } \\
\text { type design; (c) the issuance of a certificate required by } \\
\text { national laws to declare that compliance or conformity } \\
\text { has been found with standards in accordance with items } \\
\text { (a) or (b) above [4]. Certified software is software that is } \\
\text { legally recognized to be compliant with some } \\
\text { certification criteria by a certification authority as it is } \\
\text { used in a particular system context. }\end{array}$ & $\begin{array}{l}5.4 .3 \\
9 \\
10 \\
11.1\end{array}$ \\
\hline $\begin{array}{l}\text { Certification } \\
\text { Requirement }\end{array}$ & $\begin{array}{l}\text { A requirement that needs to be fulfilled in order for a } \\
\text { product, service, organization, or person to be certified } \\
\text { by a certification authority according to some } \\
\text { certification criteria. }\end{array}$ & 2.1 .1 \\
\hline $\begin{array}{l}\text { Derived } \\
\text { Requirement }\end{array}$ & $\begin{array}{l}\text { Additional requirement resulting from the software } \\
\text { development process, which may not be directly } \\
\text { traceable to higher level requirements [4]. Derived } \\
\text { requirements often appear in the form of implementation } \\
\text { constraints [4]. }\end{array}$ & 5.1 .1 \\
\hline
\end{tabular}




\begin{tabular}{|c|c|c|}
\hline Concept & Description & $\begin{array}{l}\text { RTCA DO- } \\
\text { 178B Section }\end{array}$ \\
\hline Deviation & $\begin{array}{l}\text { Difference in the output or execution of a process, such } \\
\text { as the design or implementation processes, from its plan. }\end{array}$ & 8.2 \\
\hline $\begin{array}{l}\text { Hardware / Software } \\
\text { Interface }\end{array}$ & $\begin{array}{l}\text { The boundary at which software and hardware directly } \\
\text { communicate with each other. This is usually done at } \\
\text { the bit or byte level. }\end{array}$ & $\begin{array}{l}6.4 .3 \\
11.1 \\
11.9\end{array}$ \\
\hline Traceability & $\begin{array}{l}\text { The evidence of an association between items, such as } \\
\text { between process outputs, between an output and its } \\
\text { originating process, or between a requirement and its } \\
\text { implementation [4]. Horizontal traceability expresses } \\
\text { relationship between items in different phases of the } \\
\text { development life cycle, such as the relationship between } \\
\text { a design component and software requirements. On the } \\
\text { other hand, vertical traceability expresses relationship } \\
\text { between items in the same phase of the development life } \\
\text { cycle, such as the relationship between two software } \\
\text { requirements. }\end{array}$ & $\begin{array}{l}5.3 .1 \\
5.5\end{array}$ \\
\hline
\end{tabular}

Table 14: Safety-related concepts that are classified as "primarily certification".

\section{C.7 Primarily Design Concepts}

\begin{tabular}{|l|l|l|}
\hline \multicolumn{1}{|c|}{ Concept } & \multicolumn{1}{|c|}{ Description } & \multicolumn{1}{|c|}{$\begin{array}{c}\text { RTCA DO- } \\
\text { 178B Section }\end{array}$} \\
\hline COTS Software & $\begin{array}{l}\text { Commercially available applications sold by vendors } \\
\text { through public catalogue listings. COTS software is not } \\
\text { intended to be customized or enhanced. Contract- } \\
\text { negotiated software developed for a specific application } \\
\text { is not COTS software [4]. }\end{array}$ & $\begin{array}{l}2.4 \\
\text { Compacted } \\
\text { Expression }\end{array}$ \\
\hline
\end{tabular}




\begin{tabular}{|c|c|c|}
\hline Concept & Description & $\begin{array}{l}\text { RTCA DO- } \\
\text { 178B Section }\end{array}$ \\
\hline Complexity & $\begin{array}{l}\text { The degree to which a system, software, or component } \\
\text { has a design and implementation that is difficult to } \\
\text { understand and verify [16]. Increasing the complexity } \\
\text { level of software makes it harder to formulate its overall } \\
\text { behaviour, even when given almost complete } \\
\text { information about its atomic components and their inter- } \\
\text { relations [17]. It also makes it harder to verify the } \\
\text { software design and the fulfilment of the safety } \\
\text { objectives [4]. Examples of complexity measures } \\
\text { include level of nesting, cyclomatic complexity, } \\
\text { conditional structure, unconditional branches, number of } \\
\text { entries into a code component, and number of exits from } \\
\text { a code component. }\end{array}$ & $\begin{array}{l}5.2 .2 \\
6.3 .4 \\
11.7\end{array}$ \\
\hline Coupling & $\begin{array}{l}\text { Coupling is a factor of the inter-module complexity of } \\
\text { software [18], which represents the strength of } \\
\text { connection between two modules [19], [20]. Myers } \\
\text { identified several types of coupling in [21], namely, } \\
\text { content coupling, common coupling, external coupling, } \\
\text { control coupling, stamp coupling, and data coupling. }\end{array}$ & 11.8 \\
\hline Data Alias & $\begin{array}{l}\text { In software, a data alias is a name for a software } \\
\text { variable, which is also accessible through another } \\
\text { different name. Essentially, the same data is known and } \\
\text { accessed under different names. Although allowed by } \\
\text { programming languages, this technique is generally } \\
\text { avoided in safety-critical software as they may introduce } \\
\text { confusion. }\end{array}$ & 11.7 \\
\hline
\end{tabular}




\begin{tabular}{|c|c|c|}
\hline Concept & Description & $\begin{array}{l}\text { RTCA DO- } \\
\text { 178B Section }\end{array}$ \\
\hline Deactivated Code & $\begin{array}{l}\text { Executable object code (or data) which by design is } \\
\text { either (a) not intended to be executed (code) or used } \\
\text { (data), for example, a part of a previously developed } \\
\text { software component, or (b) is only executed (code) or } \\
\text { used (data) in certain configurations of the target } \\
\text { computer environment, for example, code that is } \\
\text { enabled by a hardware pin selection or software } \\
\text { programmed option [4]. }\end{array}$ & $\begin{array}{l}4.2 \\
5.4 .3 \\
11.10\end{array}$ \\
\hline Design by Contract & $\begin{array}{l}\text { Design by contract is a technique for software } \\
\text { development where inter-module interactions are } \\
\text { constrained by an agreement, or contract, which defines } \\
\text { the requirements and obligations of each interacting } \\
\text { software entity. A contract is composed of three parts } \\
\text { [22]: (a) class (module) invariants, which define } \\
\text { conditions that are always true (in steady-state), (b) } \\
\text { operation preconditions, which define conditions that } \\
\text { are true before calling the operation, and (c) operation } \\
\text { postconditions, which define conditions that are true } \\
\text { after an operation finishes execution. }\end{array}$ & \\
\hline Dynamic Memory & $\begin{array}{l}\text { Memory that is allocated during the execution of the } \\
\text { software through special calls to the underlying } \\
\text { operating system, rather than at compile-time by pre- } \\
\text { reserving its memory space. The use of dynamic } \\
\text { memory is generally avoided in safety-critical software. } \\
\text { A dynamic object is an object that resides in dynamic } \\
\text { memory space. }\end{array}$ & 11.7 \\
\hline Error & $\begin{array}{l}\text { A software error is a mistake in its requirements, design, } \\
\text { or code [4]. }\end{array}$ & 4.2 \\
\hline Error Prevention & $\begin{array}{l}\text { A technique that attempts to avoid or prevent the } \\
\text { occurrence of errors. Error prevention and error } \\
\text { avoidance are used interchangeably. }\end{array}$ & $\begin{array}{l}4.2 \\
4.4\end{array}$ \\
\hline
\end{tabular}




\begin{tabular}{|c|c|c|}
\hline Concept & Description & $\begin{array}{l}\text { RTCA DO- } \\
\text { 178B Section }\end{array}$ \\
\hline $\begin{array}{l}\text { Field-Loadable } \\
\text { Software }\end{array}$ & $\begin{array}{l}\text { Field-loadable software refers to software or data tables } \\
\text { that can be loaded without removing the system or } \\
\text { equipment from its installation [4]. }\end{array}$ & $\begin{array}{l}2.5 \\
6.4 .3 \\
11.1\end{array}$ \\
\hline Formal Method & $\begin{array}{l}\text { A formal method of software development is a process } \\
\text { for developing software that exploits the power of } \\
\text { mathematical notation and mathematical proofs [23]. It } \\
\text { involves the use of formal logic, discrete mathematics, } \\
\text { and computer readable languages to improve the } \\
\text { specification and verification of software [4]. }\end{array}$ & 12.3 .1 \\
\hline Loader & $\begin{array}{l}\text { A hardware or software that is used to load field- } \\
\text { loadable software. }\end{array}$ & 2.5 \\
\hline Partitioning & $\begin{array}{l}\text { Software partitioning is a technique for providing } \\
\text { isolation between functionally independent software } \\
\text { components to contain and/or isolate faults and } \\
\text { potentially reduce the effort of the software verification } \\
\text { process. It prevents specific interactions and cross- } \\
\text { coupling interference [4]. }\end{array}$ & $\begin{array}{l}2.1 .1 \\
2.3 .1 \\
5.2 .2 \\
6.3 .3 \\
11.3 \\
11.9 \\
11.10\end{array}$ \\
\hline $\begin{array}{l}\text { Previously } \\
\text { Developed Software }\end{array}$ & $\begin{array}{l}\text { Software that was developed in a previous project. It } \\
\text { may or may not have been previously certified for use in } \\
\text { one or more systems. }\end{array}$ & $\begin{array}{l}11.1 \\
11.3 \\
12.1\end{array}$ \\
\hline Recursion & $\begin{array}{l}\text { Recursion is a software implementation approach to } \\
\text { solve a problem by breaking it into a smaller problem } \\
\text { that is easier to solve. Recursion is generally avoided in } \\
\text { airborne and safety-critical software due to their high } \\
\text { demand of resources. }\end{array}$ & $\begin{array}{l}6.3 .3 \\
11.7\end{array}$ \\
\hline
\end{tabular}




\begin{tabular}{|l|l|l|}
\hline \multicolumn{1}{|c|}{ Concept } & \multicolumn{1}{|c|}{ Description } & \multicolumn{1}{|c|}{$\begin{array}{c}\text { RTCA DO- } \\
\text { 178B Section }\end{array}$} \\
\hline Simulator & $\begin{array}{l}\text { A device that enables the operator to reproduce or } \\
\text { represent under test conditions phenomena likely to } \\
\text { occur in actual performance [13]. A simulator may be an } \\
\text { actual device, computer program, or a system, which } \\
\text { interfaces to other software or hardware system in the } \\
\text { same manner as the actual devices, which will be } \\
\text { eventually used in the final system. Simulators are often } \\
\text { used when testing software. }\end{array}$ & 12.3 .5 \\
\hline Software Patch & $\begin{array}{l}\text { A modification to an object program, in which one or } \\
\text { more of the planned steps of re-compiling, re- } \\
\text { assembling or re-linking is bypassed. This does not } \\
\text { include identifiers embedded in the software product, } \\
\text { for example, part numbers and checksums [4]. }\end{array}$ & 5.4 .3 \\
\hline State-Related & \begin{tabular}{l} 
Related to a state machine or its states or transitions. \\
\hline
\end{tabular} & 6.4 .2 .1 \\
\hline
\end{tabular}

Table 15: Safety-related concepts that are classified as "primarily design".

\section{C.8 Primarily Configuration Concepts}

\begin{tabular}{|l|l|l|}
\hline \multicolumn{1}{|c|}{ Concept } & \multicolumn{1}{|c|}{ Description } & \multicolumn{1}{|c|}{$\begin{array}{c}\text { RTCA DO- } \\
\text { 178B Section }\end{array}$} \\
\hline Configuration & $\begin{array}{l}\text { A system can have multiple configurations, not all of } \\
\text { which are intended to be used in every application. } \\
\text { Therefore, a configuration represents a set of enabled } \\
\text { and disabled functionality. This can lead to deactivated } \\
\text { code that cannot be executed or data that is not used [4]. }\end{array}$ & 5.4 .3 \\
\hline $\begin{array}{l}\text { Option-Selectable } \\
\text { Software }\end{array}$ & $\begin{array}{l}\text { Software that contains software-programmed options, } \\
\text { which may be configured by the user to produce } \\
\text { different possible configurations. }\end{array}$ & 2.4 \\
\hline
\end{tabular}




\begin{tabular}{|l|l|l|}
\hline \multicolumn{1}{|c|}{ Concept } & \multicolumn{1}{|c|}{ Description } & \multicolumn{1}{c|}{$\begin{array}{c}\text { RTCA DO- } \\
\text { 178B Section }\end{array}$} \\
\hline User-Modifiable & Software that is designed to be modifiable by its users. & 2.4 \\
Software & Examples include a single memory bit used to select one & 4.2 \\
& of two equipment options, a table of messages, or a & 5.2 .3 \\
& memory area that can be programmed, compiled, and & 11.1 \\
& linked for aircraft maintenance functions [4]. & \\
\hline
\end{tabular}

Table 16: Safety-related concepts that are classified as "primarily configuration". 


\section{Appendix D Conceptual Model-Concept Relationships}

\begin{tabular}{|c|c|c|}
\hline Source End & Relationship Analysis & Destination End \\
\hline $\begin{array}{l}\text { Requirement } \\
\qquad\left[0 .{ }^{*}\right]\end{array}$ & $\begin{array}{l}\text { Is Requirement Of: Each Requirement may be } \\
\text { traceable to zero or more higher-level Requirements. } \\
\text { Conversely, a Requirement may have zero or more } \\
\text { lower-level Requirements (traceable to it). }\end{array}$ & $\begin{array}{l}\text { Requirement } \\
\qquad\left[0 . .^{*}\right]\end{array}$ \\
\hline $\begin{array}{l}\text { Deviation } \\
{\left[0 . .^{*}\right]}\end{array}$ & $\begin{array}{l}\text { References: Each Deviation must deviate from at } \\
\text { least one, potentially more, Requirement. Moreover, } \\
\text { there may exist more than one Deviation from a } \\
\text { particular Requirement. However, not every } \\
\text { Requirement may have Deviations from it, which } \\
\text { would be the case when the design fully conforms to } \\
\text { the Requirements. }\end{array}$ & $\begin{array}{l}\text { Requirement } \\
\qquad\left[1 .{ }^{*}\right]\end{array}$ \\
\hline ImplementationStyle & $\begin{array}{l}\text { Is Child Class Of: Each ImplementationStyle is a } \\
\text { Style. }\end{array}$ & Style \\
\hline $\begin{array}{l}\text { ImplementationStyle } \\
\qquad\left[0 .{ }^{*}\right]\end{array}$ & $\begin{array}{l}\text { References: Each ImplementationStyle may } \\
\text { conform to, or deviate from, zero or more } \\
\text { Requirements. Conversely, a Requirement may } \\
\text { require zero or more ImplementationStyles. In the } \\
\text { case where an ImplementationStyle is not associated } \\
\text { with any Requirements, the ImplementationStyle } \\
\text { signifies a design decision rather than an obligation } \\
\text { or a requirement. }\end{array}$ & $\begin{array}{l}\text { Requirement } \\
\qquad\left[0 .{ }^{*}\right]\end{array}$ \\
\hline BehaviouralStyle & Is Child Class Of: Each BehaviouralStyle is a Style. & Style \\
\hline
\end{tabular}




\begin{tabular}{|c|c|c|}
\hline Source End & Relationship Analysis & Destination End \\
\hline $\begin{array}{c}\text { BehaviouralStyle } \\
{\left[0 .{ }^{*}\right]}\end{array}$ & $\begin{array}{l}\text { References: Each BehaviouralStyle may conform to, } \\
\text { or deviate from, zero or more Requirements. } \\
\text { Conversely, a Requirement may require zero or more } \\
\text { BehaviouralStyles. In the case where a } \\
\text { BehaviouralStyle is not associated with any } \\
\text { Requirements, the BehaviouralStyle signifies a } \\
\text { design decision rather than an obligation or a } \\
\text { requirement. }\end{array}$ & $\begin{array}{c}\text { Requirement } \\
{\left[0 .{ }^{*}\right]}\end{array}$ \\
\hline $\begin{array}{l}\text { Nature } \\
{\left[0 . .^{*}\right]}\end{array}$ & $\begin{array}{l}\text { References: A Nature may have been used solely as } \\
\text { a design decision, in which case it is not associated } \\
\text { with any Requirements, or it may have been used to } \\
\text { conform to one or more Requirements. Conversely, a } \\
\text { Requirement may exist but not cause any Natures, or } \\
\text { it may cause one or more Natures. }\end{array}$ & $\begin{array}{c}\text { Requirement } \\
{\left[0 .{ }^{*}\right]}\end{array}$ \\
\hline $\begin{array}{c}\text { Rationale } \\
{\left[0 . .^{*}\right]}\end{array}$ & $\begin{array}{l}\text { References: Each Rationale must be associated with } \\
\text { at least one, potentially more, Requirement. } \\
\text { Moreover, there may exist more than one Rationale } \\
\text { associated with a particular Requirement. However, } \\
\text { not every Requirement may have Rationales } \\
\text { associated with it. However, such a case is } \\
\text { uncommon because it would mean that there are no } \\
\text { design elements traceable to this Requirement. }\end{array}$ & $\begin{array}{c}\text { Requirement } \\
\qquad\left[1 .{ }^{*}\right]\end{array}$ \\
\hline Reaction & Is Child Class Of: Each Reaction is an Event. & Event \\
\hline $\begin{array}{c}\text { Reaction } \\
{\left[0 .{ }^{*}\right]}\end{array}$ & $\begin{array}{l}\text { Is Consequence Of: Each Reaction is a consequence } \\
\text { of one or more Events because it is executed in } \\
\text { response to the Events. However, each Event may } \\
\text { not cause any reactions at all, or it may cause one or } \\
\text { several Reactions. Since Reactions are Events by } \\
\text { inheritance, then a terminal Reaction, which is the } \\
\text { last Reaction in a chain of Reactions, does not cause } \\
\text { any more Reactions. }\end{array}$ & $\begin{array}{l}\text { Event } \\
{\left[1 . .^{*}\right]}\end{array}$ \\
\hline
\end{tabular}




\begin{tabular}{|c|c|c|}
\hline Source End & Relationship Analysis & Destination End \\
\hline $\begin{array}{c}\text { SafetyCritical } \\
{\left[1 . *^{*}\right]}\end{array}$ & $\begin{array}{l}\text { Triggers: A SafetyCritical entity may trigger zero or } \\
\text { or more Events. A particular Event may not be } \\
\text { triggered by any SafetyCritical entity, or it may be } \\
\text { triggered by one or more SafetyCritical entities. }\end{array}$ & $\begin{array}{l}\text { Event } \\
{\left[0 .{ }^{*}\right]}\end{array}$ \\
\hline $\begin{array}{c}\text { Partition } \\
{\left[0 . *^{*}\right]}\end{array}$ & $\begin{array}{l}\text { References: A Partition may exist to fulfill one or } \\
\text { more Requirements, or it may exist as a design } \\
\text { decision to isolate functionally independent elements } \\
\text { such that a failure in one component does not cause } \\
\text { the other to fail. Conversely, a Requirement may or } \\
\text { may not require one or more Partitions to be } \\
\text { performed. }\end{array}$ & $\begin{array}{c}\text { Requirement } \\
{\left[0 .^{*}\right]}\end{array}$ \\
\hline $\begin{array}{c}\text { Partition } \\
{\left[0 .{ }^{*}\right]}\end{array}$ & $\begin{array}{l}\text { Is Partitioned From: By definition, a Partition is } \\
\text { always Partitioned from one or more SafetyCritical } \\
\text { entities. However, a SafetyCritical entity may not } \\
\text { necessarily have one or more Partitions from it. }\end{array}$ & $\begin{array}{c}\text { SafetyCritical } \\
{\left[1 . *^{*}\right]}\end{array}$ \\
\hline $\begin{array}{c}\text { Handler } \\
{\left[0 . *^{*}\right]}\end{array}$ & $\begin{array}{l}\text { Handles: A Handler handles at least one Event, and } \\
\text { it usually handles more than one Event. However, } \\
\text { one or more Events may not necessarily be handled } \\
\text { by a Handler. The latter case may occur for Events } \\
\text { that are not of interest in the system, such as non- } \\
\text { safety-critical events. In addition, it usually occurs } \\
\text { for many Reactions, which are Events by } \\
\text { inheritance. }\end{array}$ & $\begin{array}{l}\text { Event } \\
{\left[1 . .^{*}\right]}\end{array}$ \\
\hline $\begin{array}{c}\text { Handler } \\
{\left[0 . *^{*}\right]}\end{array}$ & $\begin{array}{l}\text { Performs: A Handler performs one or more } \\
\text { Reactions. However, a Reaction may not necessarily } \\
\text { be performed by a Handler, or it may be performed } \\
\text { by one or more Handlers. }\end{array}$ & $\begin{array}{c}\text { Reaction } \\
{\left[1 .{ }^{*}\right]}\end{array}$ \\
\hline
\end{tabular}




\begin{tabular}{|c|c|c|}
\hline Source End & Relationship Analysis & Destination End \\
\hline $\begin{array}{c}\text { Monitor } \\
{\left[0 . .^{*}\right]}\end{array}$ & $\begin{array}{l}\text { Monitors: A Monitor monitors one or more } \\
\text { SafetyCritical entities. However, not every } \\
\text { SafetyCritical entity is monitored by a monitor. It is } \\
\text { also possible for a SafetyCritical entity to be } \\
\text { monitored by more than one Monitor. }\end{array}$ & $\begin{array}{c}\text { SafetyCritical } \\
{\left[1 . *^{*}\right]}\end{array}$ \\
\hline $\begin{array}{c}\text { Monitor } \\
{\left[0 . *^{*}\right]}\end{array}$ & $\begin{array}{l}\text { Detects: A Monitor detects at least, but usually more } \\
\text { than, one Event. However, an Event may go } \\
\text { undetected by Monitors, or it may be detected by one } \\
\text { or more Monitors. }\end{array}$ & $\begin{array}{l}\text { Event } \\
{\left[1 . *^{*}\right]}\end{array}$ \\
\hline $\begin{array}{c}\text { Monitor } \\
{\left[1 .{ }^{*}\right]}\end{array}$ & $\begin{array}{l}\text { Notifies: Each Handler is notified by at least one } \\
\text { Monitor. However, some Monitors may not } \\
\text { necessarily notify any Handlers, and a Monitor may } \\
\text { notify more than one Handler. }\end{array}$ & $\begin{array}{c}\text { Handler } \\
{\left[0 .{ }^{*}\right]}\end{array}$ \\
\hline $\begin{array}{c}\text { Simulator } \\
{\left[0 . *^{*}\right]}\end{array}$ & $\begin{array}{l}\text { Simulates: A Simulator simulates at least one } \\
\text { SafetyCritical entity. A SafetyCritical entity may not } \\
\text { have any Simulators, or it may have one or more } \\
\text { Simulators. For example, a radar may have two } \\
\text { simulators, with each one simulating the radar's } \\
\text { behaviour under different environmental conditions. } \\
\text { Another example is having two different versions for } \\
\text { a particular Simulator. }\end{array}$ & $\begin{array}{c}\text { SafetyCritical } \\
\left.\qquad 1 . .^{*}\right]\end{array}$ \\
\hline $\begin{array}{c}\text { Strategy } \\
{[0 . .1]}\end{array}$ & $\begin{array}{l}\text { Describes Design Of: A Strategy describes the } \\
\text { design of one or more SafetyCritical entities. In } \\
\text { addition, a SafetyCritical entity's design may, or } \\
\text { may not, be described by a Strategy. }\end{array}$ & $\begin{array}{c}\text { SafetyCritical } \\
{\left[1 . *^{*}\right]}\end{array}$ \\
\hline $\begin{array}{l}\text { Formalism } \\
\qquad[0 . .1]\end{array}$ & $\begin{array}{l}\text { Describes Formalism Of: A Formalism describes } \\
\text { the formalism of one or more SafetyCritical entities. } \\
\text { In addition, a SafetyCritical entity's formalism may, } \\
\text { or may not, be described by a Formalism. }\end{array}$ & $\begin{array}{c}\text { SafetyCritical } \\
{[1 . . *]}\end{array}$ \\
\hline
\end{tabular}




\begin{tabular}{|c|c|c|}
\hline Source End & Relationship Analysis & Destination End \\
\hline $\begin{array}{c}\text { Complexity } \\
{[0 . .1]}\end{array}$ & $\begin{array}{l}\text { Describes Complexity Of: A Complexity describes } \\
\text { the complexity of one or more SafetyCritical entities. } \\
\text { In addition, a SafetyCritical entity's complexity may, } \\
\text { or may not, be described by a Complexity. }\end{array}$ & $\begin{array}{c}\text { SafetyCritical } \\
\qquad\left[1 . *^{*}\right]\end{array}$ \\
\hline $\begin{array}{c}\text { Interface } \\
{\left[0 . .^{*}\right]}\end{array}$ & $\begin{array}{l}\text { Is Interface For: Each Interface is for one or more } \\
\text { SafetyCritical entities or components. In addition, a } \\
\text { specific SafetyEntity may have one or more } \\
\text { Interfaces. An example of the latter case would be } \\
\text { where a subsystem has one Interface to it in each of } \\
\text { the other subsystems in the complete system. }\end{array}$ & $\begin{array}{l}\text { SafetyCritical } \\
\qquad[1 . . *]\end{array}$ \\
\hline $\begin{array}{l}\text { Concurrent } \\
{[0 . . *]}\end{array}$ & $\begin{array}{l}\text { Triggers: Each Concurrent entity may trigger zero } \\
\text { or more Events. Conversely, each Event may be } \\
\text { triggered by zero or more Concurrent entities. A } \\
\text { Concurrent entity may not trigger any Events if it is } \\
\text { passive. }\end{array}$ & $\begin{array}{l}\text { Event } \\
{\left[0 . .^{*}\right]}\end{array}$ \\
\hline $\begin{array}{c}\text { Defensive } \\
\text { [0..1] }\end{array}$ & $\begin{array}{l}\text { Performs: A Defensive entity protects against } \\
\text { unusual inputs by performing one or more Reactions } \\
\text { to such unusual inputs, or Events. However, } \\
\text { Reactions are not necessarily performed by } \\
\text { Defensive entities. }\end{array}$ & $\begin{array}{c}\text { Reaction } \\
{\left[1 .{ }^{*}\right]}\end{array}$ \\
\hline $\begin{array}{c}\text { Configurable } \\
\text { [1..1] }\end{array}$ & $\begin{array}{l}\text { Is Defaulted To: Each Configurable entity must be } \\
\text { defaulted to a particular Configuration. }\end{array}$ & $\begin{array}{l}\text { Configuration } \\
\qquad 1 . .1]\end{array}$ \\
\hline $\begin{array}{c}\text { Configurable } \\
{\left[1 . *^{*}\right]}\end{array}$ & $\begin{array}{l}\text { Is Configurable To: Each Configurable entity may } \\
\text { be configured to produce one or more } \\
\text { Configurations. In addition, each Configuration can } \\
\text { be produces by configuration one or more } \\
\text { Configurable entities in a particular way. }\end{array}$ & $\begin{array}{c}\text { Configuration } \\
{\left[1 .{ }^{*}\right]}\end{array}$ \\
\hline $\begin{array}{c}\text { Loadable } \\
\left.\qquad 1 .{ }^{*}\right]\end{array}$ & $\begin{array}{l}\text { Is Loadable On: Each Loadable entity is loadable } \\
\text { on one or more Configurable entities. Conversely, } \\
\text { every Configurable entity can be configured by } \\
\text { loading one or more Loadables on it. }\end{array}$ & $\begin{array}{c}\text { Configurable } \\
{\left[1 . *^{*}\right]}\end{array}$ \\
\hline
\end{tabular}




\begin{tabular}{|c|c|c|}
\hline Source End & Relationship Analysis & Destination End \\
\hline $\begin{array}{l}\text { Loadable } \\
\qquad[0 . *]\end{array}$ & $\begin{array}{l}\text { Requires: Loading a Loadble entity may require } \\
\text { specific base Configurations for it to be Loaded. For } \\
\text { example, loading a particular software patch may } \\
\text { require pre-loading earlier patches. However, there } \\
\text { may not be such a requirement if the patch is a } \\
\text { complete and comprehensive patch, rather than an } \\
\text { incremental patch. Conversely, not every } \\
\text { Configuration is required by Loadable entities. }\end{array}$ & $\begin{array}{c}\text { Configuration } \\
\qquad\left[0 . *^{*}\right]\end{array}$ \\
\hline $\begin{array}{c}\text { Loadable } \\
\qquad\left[1 .{ }^{*}\right]\end{array}$ & $\begin{array}{l}\text { Produces: A Configuration may be produced by } \\
\text { loading a Loadable. A Loadable may produce more } \\
\text { than Configuration if loaded on different base } \\
\text { Configurations. For a Configuration to be produced, } \\
\text { at least one Loadable must be loaded. }\end{array}$ & $\begin{array}{l}\text { Configuration } \\
\qquad\left[1 . *^{*}\right]\end{array}$ \\
\hline $\begin{array}{c}\text { Configurator } \\
{\left[1 .{ }^{*}\right]}\end{array}$ & $\begin{array}{l}\text { Configures: A Configurator configures one or more } \\
\text { Configurable entities. A Configurable entity may be } \\
\text { configured by more than one Configurator, such as } \\
\text { the case where the Configurators configure different } \\
\text { aspects of the Configurable entity. }\end{array}$ & $\begin{array}{l}\text { Configurable } \\
\qquad[1 . . *]\end{array}$ \\
\hline $\begin{array}{c}\text { Configurator } \\
\qquad\left[1 . *^{*}\right]\end{array}$ & $\begin{array}{l}\text { Loads: A Configurator loads one or more Loadables. } \\
\text { In addition, a Loadable is loaded by one or more } \\
\text { Configurators. }\end{array}$ & $\begin{array}{l}\text { Loadable } \\
\left.\qquad 1 . .^{*}\right]\end{array}$ \\
\hline $\begin{array}{l}\text { Comparator } \\
\quad[1 . .1]\end{array}$ & $\begin{array}{l}\text { Compares: A Comparator compares the outputs of } \\
\text { at least two Replicated entities. The output of a } \\
\text { Replicated entity is compared by exactly one } \\
\text { Comparator. }\end{array}$ & $\begin{array}{c}\text { Replicated } \\
\quad\left[2 .{ }^{*}\right]\end{array}$ \\
\hline $\begin{array}{l}\text { ReplicationGroup } \\
\qquad[1 . .1]\end{array}$ & $\begin{array}{l}\text { Owns: Each ReplicationGroup has exactly one } \\
\text { Comparator. }\end{array}$ & $\begin{array}{c}\text { Comparator } \\
\quad[1 . .1]\end{array}$ \\
\hline $\begin{array}{l}\text { ReplicationGroup } \\
\qquad[1 . .1]\end{array}$ & $\begin{array}{l}\text { Owns: Each ReplicationGroup has at least two } \\
\text { Replicated entities. }\end{array}$ & $\begin{array}{l}\text { Replicated } \\
\qquad\left[2 . .^{*}\right]\end{array}$ \\
\hline
\end{tabular}

Table 17: Analysis of conceptual model concept relationships. 


\section{Appendix E Gomaa's Class Classification}

This section identifies Gomaa's class classifications as described in [7]. Each classification is represented by a unique stereotype. The stereotypes are shown in Figure 16.

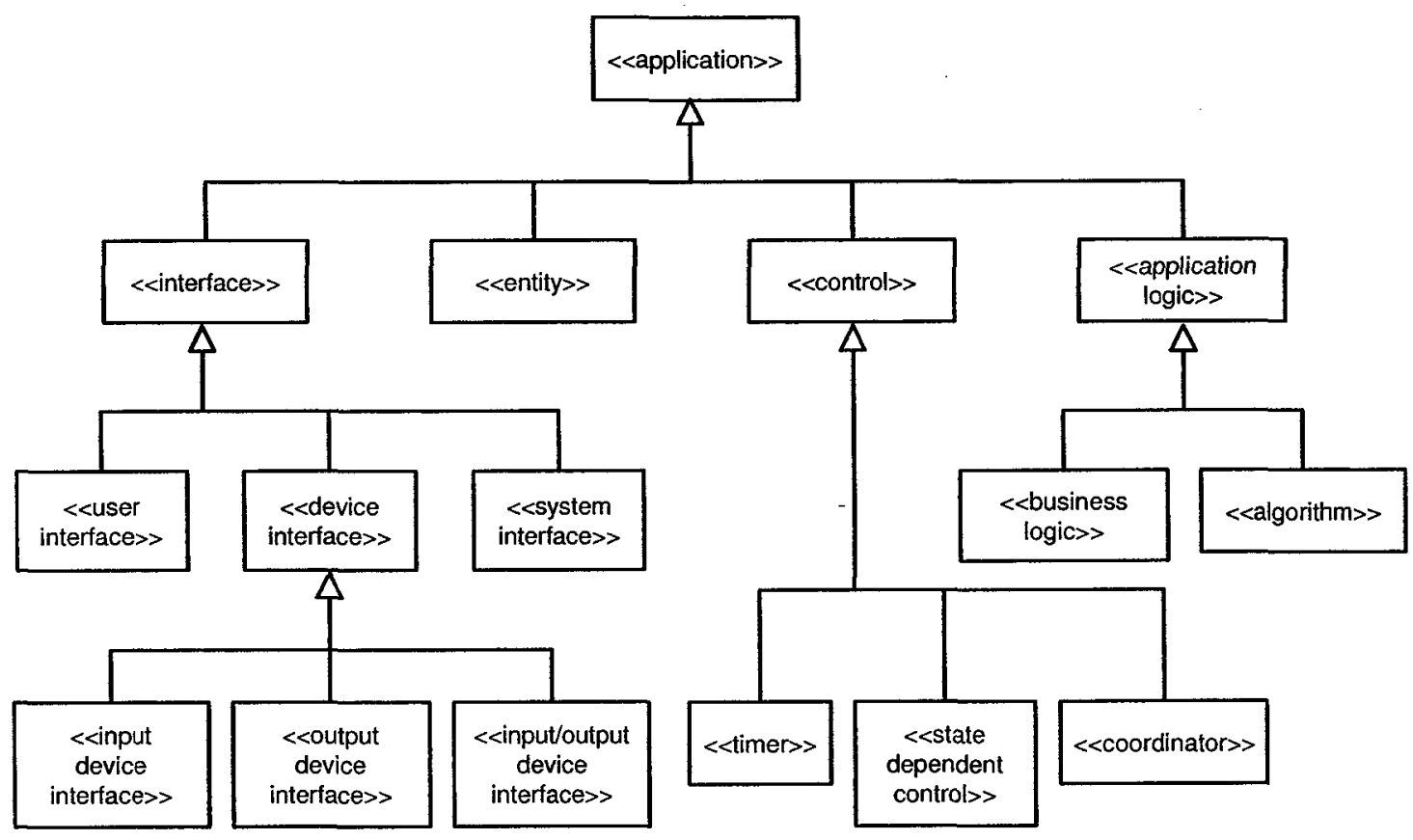

Figure 16: Gomaa's classification of application classes using stereotypes [7]. 


\section{Appendix F Assessing Existing Profiles based on the}

\section{Information Requirements}

\section{F.1 Quality of Service and Fault Tolerance OMG Profile (discussed in}

\section{Section 4.1)}

\begin{tabular}{|c|c|c|}
\hline IREQ \# & Fulfilled & Comment \\
\hline IREQ 1 & Yes & Use $<<$ QoSContext $\gg>$ stereotype \\
\hline IREQ 2 & Yes & Use $<<$ FTFaultTolerantDomain $>>$ stereotype \\
\hline IREQ 3 & No & $\begin{array}{l}\text { Can reuse }<<\text { QoSContext } \gg>\text { stereotype, but then cannot differentiate it } \\
\text { from IREQ } 1\end{array}$ \\
\hline IREQ 4 & No & $\begin{array}{l}\text { Can reuse }<<\text { QoSContext } \gg>\text { stereotype, but then cannot differentiate it } \\
\text { from IREQ } 1\end{array}$ \\
\hline IREQ 5 & No & $\begin{array}{l}\text { Can reuse }<<\text { QoSContext } \gg>\text { stereotype, but then cannot differentiate it } \\
\text { from IREQ } 1\end{array}$ \\
\hline IREQ 6 & No & $\begin{array}{l}\text { Can reuse }<<\text { QoSContext } \gg>\text { stereotype, but then cannot differentiate it } \\
\text { from IREQ } 1\end{array}$ \\
\hline IREQ 7 & No & \\
\hline IREQ 8 & No & \\
\hline IREQ 9 & Yes & Use $<<$ QoSConstraint $>>$ stereotype and its child classes \\
\hline IREQ 10 & No & \\
\hline IREQ 11 & No & \\
\hline IREQ 12 & No & \\
\hline IREQ 13 & No & \\
\hline IREQ 14 & Yes & Use $<<$ QoSLevel $>>$ and $<<$ QoSTransition $>>$ stereotypes \\
\hline IREQ 15 & No & \\
\hline IREQ 16 & No & \\
\hline IREQ 17 & No & \\
\hline IREQ 18 & No & \\
\hline IREQ 19 & No & \\
\hline IREQ 20 & No & \\
\hline IREQ 21 & Yes & Use $<<$ QoSDimension $>>$ stereotype \\
\hline IREQ 22 & No & \\
\hline IREQ 23 & Yes & Use $<<$ Initiate $>>$ stereotype \\
\hline IREQ 24 & Yes & Use $<<$ QoSDimension $>>$ \\
\hline IREQ 25 & Yes & Use $<<$ Asset $\gg>$ stereotype, but it is not suitable for all uses \\
\hline IREQ 26 & Yes & Use $<<$ QoSCharacteristic $>>$ and $<<$ QoSDimension $\gg>$ stereotypes \\
\hline IREQ 27 & No & \\
\hline
\end{tabular}




\begin{tabular}{|c|c|c|}
\hline IREQ \# & Fulfilled & Comment \\
\hline IREQ 28 & No & \\
\hline IREQ 29 & Yes & $\begin{array}{l}\text { Use } \quad<\text { FTReplicationStyle }>>\text { and } \quad<<\text { FTFaultTolerantDomain }>> \\
\text { stereotypes }\end{array}$ \\
\hline IREQ 30 & No & \\
\hline IREQ 31 & Yes & $\begin{array}{l}\text { Use } \quad<\text { FTReplicationStyle }>>\text { and } \quad<<\text { FTFaultTolerantDomain }>> \\
\text { stereotypes }\end{array}$ \\
\hline IREQ 32 & No & \\
\hline IREQ 33 & No & \\
\hline IREQ 34 & Yes & $\begin{array}{l}\begin{array}{l}\text { The simulation parameters may be specified by using the } \\
<<\text { QoSValue }>>\text { stereotype and its child classes }\end{array} \\
\end{array}$ \\
\hline IREQ 35 & No & \\
\hline IREQ 36 & No & \\
\hline IREQ 37 & No & \\
\hline IREQ 38 & Yes & $\begin{array}{l}\text { Can use }<<\text { QoSValue }>>\text { stereotype, but it may get confusing with others } \\
\text { such as IREQ } 40\end{array}$ \\
\hline IREQ 39 & No & \\
\hline IREQ 40 & Yes & Use $<<$ QoSValue $>>$ stereotype and its child classes \\
\hline IREQ 41 & No & \\
\hline IREQ 42 & No & \\
\hline IREQ 43 & No & \\
\hline IREQ 44 & No & \\
\hline IREQ 45 & No & \\
\hline IREQ 46 & No & \\
\hline IREQ 47 & No & \\
\hline IREQ 48 & No & \\
\hline IREQ 49 & No & \\
\hline IREQ 50 & No & \\
\hline IREQ 51 & No & \\
\hline IREQ 52 & Yes & Use Fault-Tolerance sub-profile \\
\hline IREQ 53 & Yes & $\begin{array}{l}\begin{array}{l}\text { Use Fault-Tolerance sub-profile, and } \\
\text { <<FTReplicationStyle }>>\text { stereotype }\end{array} \\
\text { < }\end{array}$ \\
\hline IREQ 54 & Yes & $\begin{array}{l}\text { Use Fault-Tolerance sub-profile, and specifically the } \\
<<\text { FTReplicationStyle }>>\text { and }<<\text { FTFaultTolerantDomain }>>\text { stereotypes }\end{array}$ \\
\hline Total & 17 & Only 17 information requirements out of 54 are fulfilled \\
\hline
\end{tabular}

Table 18: Assessing the Quality of Service and Fault Tolerance OMG profile based on the information requirements. 
F.2 Schedulability, Performance, and Time OMG Profile (discussed in

Section 4.2)

\begin{tabular}{|c|c|c|}
\hline IREQ \# & Fulfilled & Comment \\
\hline IREQ 1 & No & \\
\hline IREQ 2 & No & \\
\hline IREQ 3 & No & \\
\hline IREQ 4 & Yes & Use $<<$ PAcontext $>>$ stereotype \\
\hline IREQ 5 & Yes & Use $<<$ SAsituation $>>$ stereotype \\
\hline IREQ 6 & No & \\
\hline IREQ 7 & No & \\
\hline IREQ 8 & No & \\
\hline IREQ 9 & No & \\
\hline IREQ 10 & No & \\
\hline IREQ 11 & No & \\
\hline IREQ 12 & No & \\
\hline IREQ 13 & No & \\
\hline IREQ 14 & No & \\
\hline IREQ 15 & No & \\
\hline IREQ 16 & No & \\
\hline IREQ 17 & No & \\
\hline IREQ 18 & No & \\
\hline IREQ 19 & No & \\
\hline IREQ 20 & Yes & Use $<<$ SAtrigger $>>$ stereotype \\
\hline IREQ 21 & No & \\
\hline IREQ 22 & Yes & Use $<<$ SAresponse $>>$ stereotype \\
\hline IREQ 23 & No & \\
\hline IREQ 24 & No & \\
\hline IREQ 25 & No & \\
\hline IREQ 26 & No & \\
\hline IREQ 27 & No & \\
\hline IREQ 28 & No & \\
\hline IREQ 29 & No & \\
\hline IREQ 30 & No & \\
\hline IREQ 31 & No & \\
\hline IREQ 32 & No & \\
\hline IREQ 33 & No & \\
\hline IREQ 34 & No & \\
\hline IREQ 35 & No & \\
\hline IREQ 36 & No & \\
\hline
\end{tabular}




\begin{tabular}{|c|c|c|}
\hline IREQ \# & Fulfilled & Comment \\
\hline IREQ 37 & No & \\
\hline IREQ 38 & No & \\
\hline IREQ 39 & No & \\
\hline IREQ 40 & No & \\
\hline IREQ 41 & Yes & Use $<<$ CRconcurrent $\gg>$ stereotype \\
\hline IREQ 42 & No & \\
\hline IREQ 43 & Yes & Use $<<$ PAresource>> and $<<$ SAresource $>>$ stereotypes \\
\hline IREQ 44 & No & \\
\hline IREQ 45 & No & \\
\hline IREQ 46 & No & \\
\hline IREQ 47 & No & \\
\hline IREQ 48 & No & \\
\hline IREQ 49 & No & \\
\hline IREQ 50 & No & \\
\hline IREQ 51 & No & \\
\hline IREQ 52 & No & \\
\hline IREQ 53 & No & \\
\hline IREQ 54 & No & \\
\hline Total & 6 & Only 6 information requirements out of 54 are fulfilled \\
\hline
\end{tabular}

Table 19: Assessing the Schedulability, Performance, and Time OMG profile based on the information requirements. 


\section{F.3 HIDOORS Profile (discussed in Section 4.3)}

\begin{tabular}{|c|c|c|}
\hline IREQ\# & Fulfilled & Comment \\
\hline IREQ 1 & No & \\
\hline IREQ 2 & No & \\
\hline IREQ 3 & No & \\
\hline IREQ 4 & Yes & Reuse $<<$ PAcontext $\gg>$ stereotype from SPT profile \\
\hline IREQ 5 & Yes & Reuse $<<$ SAsituation $>>$ stereotype from SPT profile \\
\hline IREQ 6 & No & \\
\hline IREQ 7 & No & \\
\hline IREQ 8 & No & \\
\hline IREQ 9 & No & \\
\hline IREQ 10 & No & \\
\hline IREQ 11 & No & \\
\hline IREQ 12 & No & \\
\hline IREQ 13 & No & \\
\hline IREQ 14 & No & \\
\hline IREQ 15 & No & \\
\hline IREQ 16 & No & \\
\hline IREQ 17 & No & \\
\hline IREQ 18 & No & \\
\hline IREQ 19 & No & \\
\hline IREQ 20 & Yes & $\begin{array}{l}\text { Use the }<<\text { HIEvent } \gg>\text { stereotype, or reuse }<<\text { SAtrigger }>>\text { stereotype } \\
\text { from SPT profile }\end{array}$ \\
\hline IREQ 21 & No & \\
\hline IREQ 22 & Yes & Reuse $<<$ SAresponse $>>$ stereotype from SPT profile \\
\hline IREQ 23 & No & \\
\hline IREQ 24 & No & \\
\hline IREQ 25 & No & \\
\hline IREQ 26 & No & \\
\hline IREQ 27 & No & \\
\hline IREQ 28 & No & \\
\hline IREQ 29 & No & \\
\hline IREQ 30 & No & \\
\hline IREQ 31 & No & \\
\hline IREQ 32 & No & \\
\hline IREQ 33 & No & \\
\hline IREQ 34 & No & \\
\hline IREQ 35 & No & \\
\hline IREQ 36 & No & \\
\hline IREQ 37 & No & \\
\hline IREQ 38 & No & \\
\hline
\end{tabular}




\begin{tabular}{|c|c|l|}
\hline IREQ \# & Fulfilled & \multicolumn{1}{|c|}{ Comment } \\
\hline IREQ 39 & No & \\
\hline IREQ 40 & No & \\
\hline IREQ 41 & Yes & $\begin{array}{l}\text { Use the } \\
\text { stereotype from SPT profile }\end{array}$ \\
\hline IREQ 42 & No & \\
\hline IREQ 43 & Yes & $\begin{array}{l}\text { Reuse } \text { <<PAresource>> and <<SAresource>> stereotypes from SPT } \\
\text { profile }\end{array}$ \\
\hline IREQ 44 & No & \\
\hline IREQ 45 & No & \\
\hline IREQ 46 & No & \\
\hline IREQ 47 & No & \\
\hline IREQ 48 & No & \\
\hline IREQ 49 & No & \\
\hline IREQ 50 & No & \\
\hline IREQ 51 & No & \\
\hline IREQ 52 & No & \\
\hline IREQ 53 & No & \\
\hline IREQ 54 & No & \\
\hline Total & 6 & Only 6 information requirements out of 54 are fulfilled \\
\hline
\end{tabular}

Table 20: Assessing the HIDOORS profile based on the information requirements. 


\section{F.4 Effects of Message Loss, Delay, and Corruption (discussed in}

\section{Section 4.4)}

\begin{tabular}{|c|c|c|}
\hline IREQ \# & Fulfilled & Comment \\
\hline IREQ 1 & Yes & $\begin{array}{l}\text { Use <<safe behaviour }>>\text {, }<<\text { safe dependency }>>\text {, and }<<\text { safe links }>> \\
\text { stereotypes }\end{array}$ \\
\hline IREQ 2 & No & \\
\hline IREQ 3 & No & \\
\hline IREQ 4 & No & \\
\hline IREQ 5 & No & \\
\hline IREQ 6 & No & \\
\hline IREQ 7 & No & \\
\hline IREQ 8 & No & \\
\hline IREQ 9 & Yes & Use <<guarantee $>>$ stereotype \\
\hline IREQ 10 & No & \\
\hline IREQ 11 & No & \\
\hline IREQ 12 & No & \\
\hline IREQ 13 & No & \\
\hline IREQ 14 & No & \\
\hline IREQ 15 & No & \\
\hline IREQ 16 & No & \\
\hline IREQ 17 & No & \\
\hline IREQ 18 & No & \\
\hline IREQ 19 & No & \\
\hline IREQ 20 & No & \\
\hline IREQ 21 & No & \\
\hline IREQ 22 & No & \\
\hline IREQ 23 & No & \\
\hline IREQ 24 & No & \\
\hline IREQ 25 & Yes & Use $<<$ critical $>>$ stereotype \\
\hline IREQ 26 & Yes & Use $<<$ critical $>>$ stereotype and its "level" tagged value \\
\hline IREQ 27 & No & \\
\hline IREQ 28 & Yes & Use $<<$ error handling $>>$ stereotype \\
\hline IREQ 29 & Yes & Use $<<$ containment $>>$ stereotype \\
\hline IREQ 30 & No & \\
\hline IREQ 31 & No & \\
\hline IREQ 32 & No & \\
\hline IREQ 33 & No & \\
\hline IREQ 34 & No & \\
\hline IREQ 35 & No & \\
\hline IREQ 36 & No & \\
\hline
\end{tabular}




\begin{tabular}{|c|c|c|}
\hline IREQ \# & Fulfilled & Comment \\
\hline IREQ 37 & No & \\
\hline IREQ 38 & No & \\
\hline IREQ 39 & No & \\
\hline IREQ 40 & No & \\
\hline IREQ 41 & No & \\
\hline IREQ 42 & No & \\
\hline IREQ 43 & No & \\
\hline IREQ 44 & No & \\
\hline IREQ 45 & No & \\
\hline IREQ 46 & No & \\
\hline IREQ 47 & No & \\
\hline IREQ 48 & No & \\
\hline IREQ 49 & No & \\
\hline IREQ 50 & No & \\
\hline IREQ 51 & No & \\
\hline IREQ 52 & Yes & Use <<redundancy >> stereotype \\
\hline IREQ 53 & No & \\
\hline IREQ 54 & No & \\
\hline Total & 7 & Only 7 information requirements out of 54 are fulfilled \\
\hline
\end{tabular}

Table 21: Assessing the Effects of Messages profile based on the information requirements. 


\section{F.5 Patterns for Reliability and Safety (discussed in Section 4.5)}

\begin{tabular}{|c|c|l|}
\hline IREQ \# & Fulfilled & \\
\hline IREQ 1 & No & \\
\hline IREQ 2 & No & \\
\hline IREQ 3 & No & \\
\hline IREQ 4 & No & \\
\hline IREQ 5 & No & \\
\hline IREQ 6 & No & \\
\hline IREQ 7 & No & \\
\hline IREQ 8 & No & \\
\hline IREQ 9 & No & \\
\hline IREQ 10 & No & \\
\hline IREQ 11 & No & \\
\hline IREQ 12 & No & \\
\hline IREQ 13 & No & \\
\hline IREQ 14 & No & \\
\hline IREQ 15 & No & \\
\hline IREQ 16 & No & \\
\hline IREQ 17 & No & \\
\hline IREQ 18 & No & \\
\hline IREQ 19 & No & \\
\hline IREQ 20 & No & \\
\hline IREQ 21 & No & \\
\hline IREQ 22 & No & \\
\hline IREQ 23 & No & \\
\hline IREQ 24 & No & \\
\hline IREQ 25 & No & \\
\hline IREQ 26 & Yes & Use "qosSafety" constraint \\
\hline IREQ 27 & No & \\
\hline IREQ 28 & No & \\
\hline IREQ 29 & No & Paper introduces some patterns to model this, but only for use cases \\
\hline IREQ 30 & No & \\
\hline IREQ 31 & No & \\
\hline IREQ 32 & No & \\
\hline IREQ 33 & No & \\
\hline IREQ 34 & No & \\
\hline IREQ 35 & No & \\
\hline IREQ 36 & No & \\
\hline IREQ 37 & No & \\
\hline IREQ 38 & No & \\
\hline IREQ 39 & No & \\
\hline & & \\
\hline
\end{tabular}




\begin{tabular}{|c|c|c|}
\hline IREQ \# & Fulfilled & Comment \\
\hline IREQ 40 & No & \\
\hline IREQ 41 & No & \\
\hline IREQ 42 & No & \\
\hline IREQ 43 & No & \\
\hline IREQ 43 & No & \\
\hline IREQ 46 & No & \\
\hline IREQ 47 & No & \\
\hline IREQ 48 & No & \\
\hline IREQ 49 & No & \\
\hline IREQ 50 & No & \\
\hline IREQ 51 & No & \\
\hline IREQ 52 & No & Paper introduces some patterns to model this, but only for use cases \\
\hline IREQ 53 & No & \\
\hline IREQ 54 & No & \\
\hline Total & 1 & Only 1 information requirement out of 54 is fulfilled \\
\hline
\end{tabular}

Table 22: Assessing patterns for Reliability and Safety based on the information requirements. 
F.6 Assessing Existing Profiles based on the Safety Information

Requirements-Summary (discussed in section 4.6)

\begin{tabular}{|c|c|c|c|c|c|c|}
\hline IREQ \# & $\begin{array}{c}\text { OMG } \\
\text { QoS \& } \\
\text { FT }\end{array}$ & $\begin{array}{l}\text { OMG } \\
\text { SPT }\end{array}$ & HIDOORS & $\begin{array}{l}\text { Effects of } \\
\text { Messages }\end{array}$ & Patterns & $\begin{array}{c}\text { All } \\
\text { Combined }\end{array}$ \\
\hline IREQ 1 & Yes & & & Yes & & Yes \\
\hline IREQ 2 & Yes & & & & & Yes \\
\hline \multicolumn{7}{|l|}{ IREQ 3} \\
\hline IREQ 4 & & Yes & Yes & & & Yes \\
\hline IREQ 5 & & Yes & Yes & & & Yes \\
\hline \multicolumn{7}{|l|}{ IREQ 6} \\
\hline \multicolumn{7}{|l|}{ IREQ 7} \\
\hline \multicolumn{7}{|l|}{ IREQ 8} \\
\hline IREQ 9 & Yes & & & Yes & & Yes \\
\hline \multicolumn{7}{|l|}{ IREQ 10} \\
\hline \multicolumn{7}{|l|}{ IREQ 11} \\
\hline \multicolumn{7}{|l|}{ IREQ 12} \\
\hline \multicolumn{7}{|l|}{ IREQ 13} \\
\hline IREQ 14 & Yes & & & & & Yes \\
\hline \multicolumn{7}{|l|}{ IREQ 15} \\
\hline \multicolumn{7}{|l|}{ IREQ 16} \\
\hline \multicolumn{7}{|l|}{ IREQ 17} \\
\hline \multicolumn{7}{|l|}{ IREQ 18} \\
\hline \multicolumn{7}{|l|}{ IREQ 19} \\
\hline IREQ 20 & & Yes & Yes & & & Yes \\
\hline IREQ 21 & Yes & & & & & Yes \\
\hline IREQ 22 & & Yes & Yes & & & Yes \\
\hline IREQ 23 & Yes & & & & & Yes \\
\hline IREQ 24 & Yes & & & & & Yes \\
\hline IREQ 25 & Yes & & & Yes & & Yes \\
\hline IREQ 26 & Yes & & & Yes & Yes & Yes \\
\hline \multicolumn{7}{|l|}{ IREQ 27} \\
\hline IREQ 28 & & & & Yes & & Yes \\
\hline IREQ 29 & Yes & & & Yes & & Yes \\
\hline \multicolumn{7}{|l|}{ IREQ 30} \\
\hline IREQ 31 & Yes & & & & & Yes \\
\hline \multicolumn{7}{|l|}{ IREQ 32} \\
\hline \multicolumn{7}{|l|}{ IREQ 33} \\
\hline IREQ 34 & Yes & & & & & Yes \\
\hline IREQ 35 & & & & & & \\
\hline
\end{tabular}




\begin{tabular}{|c|c|c|c|c|c|c|}
\hline IREQ \# & $\begin{array}{c}\text { OMG } \\
\text { QoS \& } \\
\text { FT }\end{array}$ & $\begin{array}{l}\text { OMG } \\
\text { SPT }\end{array}$ & HIDOORS & $\begin{array}{l}\text { Effects of } \\
\text { Messages }\end{array}$ & Patterns & $\begin{array}{c}\text { All } \\
\text { Combined }\end{array}$ \\
\hline \multicolumn{7}{|l|}{ IREQ 36} \\
\hline \multicolumn{7}{|l|}{ IREQ 37} \\
\hline IREQ 38 & Yes & & & & & Yes \\
\hline \multicolumn{7}{|l|}{ IREQ 39} \\
\hline IREQ 40 & Yes & & & & & Yes \\
\hline IREQ 41 & & Yes & Yes & & & Yes \\
\hline \multicolumn{7}{|l|}{ IREQ 42} \\
\hline IREQ 43 & & Yes & Yes & & & Yes \\
\hline \multicolumn{7}{|l|}{ IREQ 44} \\
\hline \multicolumn{7}{|l|}{ IREQ 45} \\
\hline \multicolumn{7}{|l|}{ IREQ 46} \\
\hline \multicolumn{7}{|l|}{ IREQ 47} \\
\hline \multicolumn{7}{|l|}{ IREQ 48} \\
\hline \multicolumn{7}{|l|}{ IREQ 49} \\
\hline \multicolumn{7}{|l|}{ IREQ 50} \\
\hline \multicolumn{7}{|l|}{ IREQ 51} \\
\hline IREQ 52 & Yes & & & Yes & & Yes \\
\hline IREQ 53 & Yes & & & - & & Yes \\
\hline IREQ 54 & Yes & & & & & Yes \\
\hline $\begin{array}{l}\text { Total (Max } \\
=54)\end{array}$ & 17 & 6 & 6 & 7 & 1 & 24 \\
\hline $\begin{array}{c}\text { Percentage } \\
(\%)\end{array}$ & $31 \%$ & $11 \%$ & $11 \%$ & $13 \%$ & $2 \%$ & $44 \%$ \\
\hline
\end{tabular}

Table 23: Assessment summary of existing UML profiles based on the information requirements. 


\section{Appendix G Additional UML Profile Examples}

\section{G.1 COTS Software}

The example in Figure 17 shows an aircraft navigation controller, which controls the flight of the aircraft including all auto pilot programmes.

NavigationController controls the aircraft's flight paths. Therefore, it is a safetycritical element. This is marked explicitly on the diagram by stereotyping NavigationController with a $<<$ SafetyCritical $>$ (5.2.17) stereotype. Because the failure of this system can result in conditions difficult to handle by the aircraft's crew, this class has been assigned software level C. This is indicated by the "CriticalityLevel" tagged value of the $<<$ SafetyCritical $\gg$ (5.2.17) stereotype.

NavigationController needs to know the allowed flight paths of the aircraft. In this example, SafeFlightPaths serves as the database that contains all the navigation information relevant to the currently needed flight paths. It is safety-critical because a safety-critical class, namely Navigationcontroller, depends on it. This is marked explicitly on the diagram by stereotyping it with a $<<$ SafetyCritical $>>$ (5.2.17) stereotype and assigning it a software level equal to at least that of the class that depends on it as indicated by the "CriticalityLevel" tagged. The developers of this navigation controller system decided to purchase COTS software and use it to store the flight paths. This is indicated in the diagram by stereotyping SafeFlightPaths with $<<$ Nature $>$ (5.2.13) and setting the "Kind" tagged value to "COTS". 
For the aircraft's flight paths to be meaningful, maps of the world are needed. They are necessary for verifying the safe flight paths as well as displaying them to the pilots. Unlike the flight paths, they are static information that rarely change, and therefore they are maintained as a separate class outside of SafeFlightPaths. They are maintained in CurrentlyusedworldMaps, which contains the world maps that are needed for the current flight. It is safety-critical because a safety-critical class, namely SafeFlightPaths, depends on it. This is marked explicitly on the diagram by stereotyping it with a $<$ SafetyCritical $>>$ (5.2.17) stereotype and assigning it a software level equal to at least that of the class that depends on it as indicated by the "CriticalityLevel" tagged.

This diagram shows safety-critical model elements. Hence, it is stereotyped with $<<$ SafetyContext $>>$ (5.2.1). In addition, COTS software is a crucial element of the software's certification aspects in airworthiness. Therefore, they must be declared to the certification authorities. For this reason, this diagram is stereotyped with $<<$ CertificationContext $>>(5.2 .6)$ to indicate that it contains information that is highly relevant to the certification authorities.

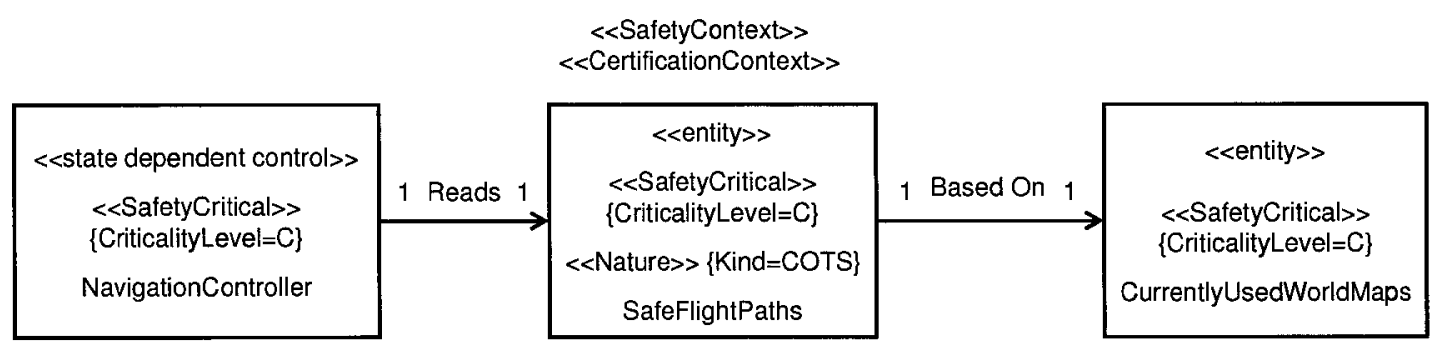

Figure 17: Aircraft's navigation controller using COTS software (structure). 


\section{G.2 Software Partitioning}

Software partitioning is a technique for providing isolation between functionally independent software components to contain and/or isolate faults and potentially reduce the effort of the software verification process. It prevents specific interactions and crosscoupling interference [1]. Its key advantages are in separating safety-critical software elements that have different safety levels, so that the failure of the less critical software does not result in the failure of the more critical software.

The example in Figure 18 shows another section of a navigation system. This system, which can be put on auto-pilot, has a steering controller that is highly safety-critical. SteeringController controls the steering of the aircraft and its movement. Therefore, it is highly safety-critical and is assigned software level B. Thus, it is stereotyped $<<$ SafetyCritical $\gg$ (5.2.17) and its "CriticalityLevel" tagged value is set to level B. Because it is highly safety-critical, it also employs defensive programming methods to protect against unsafe inputs, whether they are manual input from the pilot through PilotKeyboardinterface, or from the software's auto pilot system through AutoPilotController. SteeringController does not perform any action that requires the aircraft to fly on an altitude below 100 meters unless the aircraft is in the process of landing. Therefore, it is stereotyped with $<<$ Defensive $>$ (5.2.27) and has a "DefendableInput" tagged value set to "Altitude $<100$ m". It also does not perform any action that requires the aircraft to change its flight direction in angles of greater than 90 degrees. Thus, another instance of the "DefendableInput" tagged value is set to "Angle > 90 deg". In both cases, steeringController reports that an illegal operation has 
occurred by exeuting the ReportIl legationoperation reaction procedure, as evident from the "Reaction" tagged value that is set to "ReportlllegalOperation" .

Autopilotcontrolier is also safety-critical, but less critical than SteeringController. This is because the failure of AutoPilotController only results in inconvenience for the pilots rather than significantly compromising the level of safety. If the auto pilot feature fails, the pilots can always manually control the aircraft through PilotKeyboardinterface or some other mechanical device inputs. Therefore, AutopilotController was assigned software level D. Thus, it is stereotyped with $<<$ SafetyCritical $>$ (5.2.17) and its "CriticalityLevel" tagged value is set to level D. The interesting thing to note in this example is that AutoPilotController was partitioned from steeringController for this very reason - if the auto pilot feature fails, the steering controller can still execute correctly and ensure the safe flight of the aircraft. This is stated in the model by stereotyping AutoPilotcontroller with $<<$ Partition $>$ (5.2.18) and using its "PartitionedFrom" tagged value, set to "SteeringController", and "Explanation" tagged value, set to "Lower Criticality Level", to specify this exact information.

ConvertiblesteeringInformation is used by PilotDisplayInterface to display the current aircraft steering information. What is special about this class is that it can read the information from steeringController and convert it to appropriate units for the pilots such as conversion from metric units to imperial units, and vice-versa. Again, ConvertiblesteeringInformation was partitioned away from steeringController because it is only relevant for displaying the information to the pilots. If it fails, the 
aircraft can still resume safe flight through either manual input through PilotKeyboardinterface or auto pilot through AutoPilotController. As a result, ConvertiblesteeringInformation was stereotyped with $<<$ Partition $\gg>(5.2 .18)$ and its "PartitionedFrom" and "Explanation" tagged values were used to specify that it was partitioned from steeringcontroller because it was "Not Safety Critical".

It is clear from this discussion that this example has emphasis on safety. Therefore, the diagram was stereotyped with $<<$ SafetyContext $>>$ (5.2.1). It should also be noted that partitioning information has to be submitted to the certification authorities. Therefore, the diagram was also stereotyped with $<<$ CertificationContext $>>$ (5.2.6). Finally, we also decided to stereotype it with $<<$ DesignContext $>>(5.2 .7)$ because it is the result of design decisions on how software classes are organized with respect to safety. In fact, the partitioning concept was identified as a design concept in section C.7

One final note is that the higher the software level is, the more expensive it is to develop it. A direct positive result of the partitioning in this example is that AutopilotController and ConvertiblesteeringInformation do not have to go through the rigorous development and testing methods required for steeringcontroller, which is of level B. 


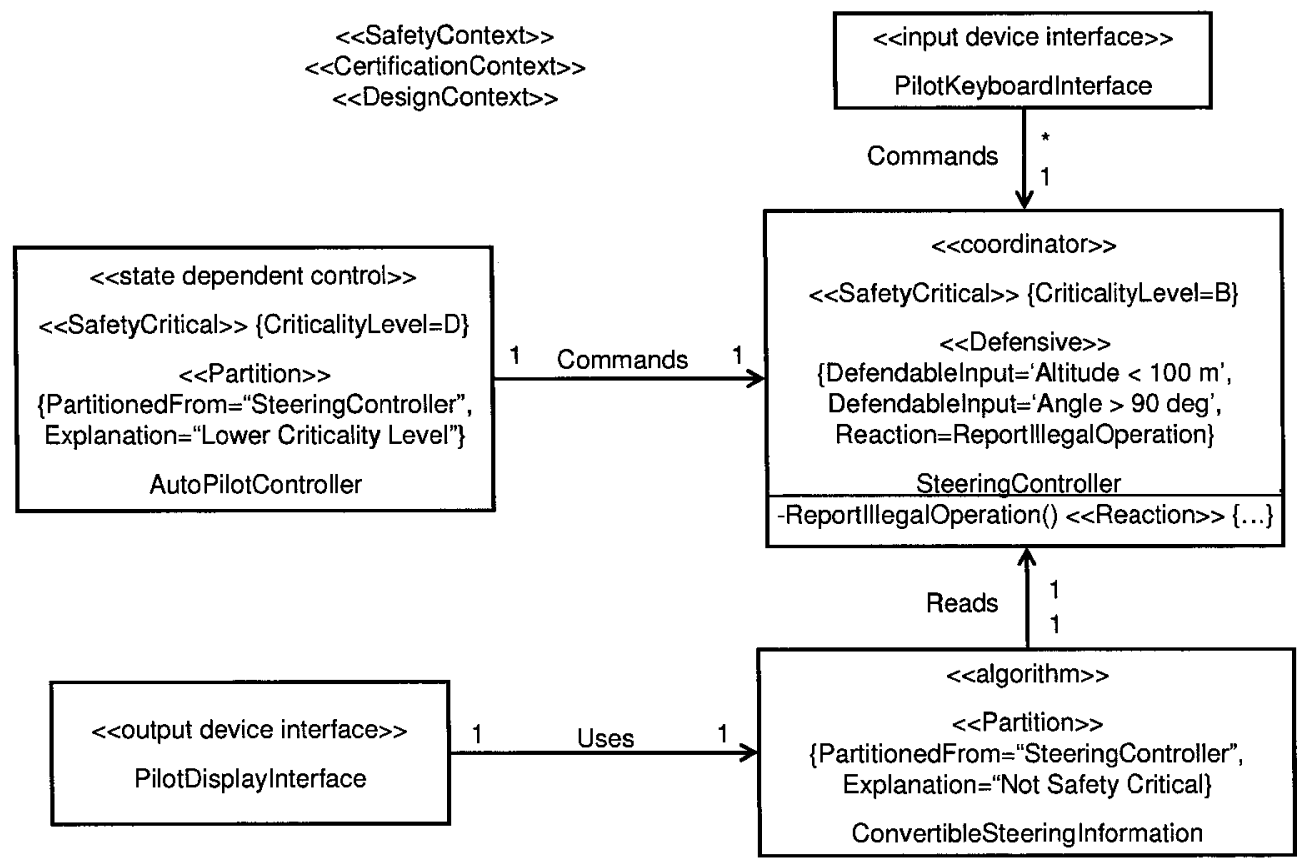

Figure 18: Aircraft steering controller (structure).

\section{G.3 Requirements and Traceability}

The example in Figure 19 shows safety-related requirements in the aircraft steering controller system presented in Figure 18. Most of the stereotypes on the classes were explained in Appendix G.2. Therefore, only new stereotypes are explained here. Such systems are generally safety-critical, and are often allocated safety requirements. As a result, this diagram has been stereotyped with $<<$ SafetyContext $>>(5.2 .1)$.

Generally, a project identifies high-level safety requirements that must be fulfilled. Then, low-level safety requirements are developed to ensure that the high-level goals are fulfilled. This diagram specifies and elaborates on a safety goal that was identified for this model, namely to ensure that the aircraft always flies in "Safe Flight Paths". This goal is captured in the diagram by stereotyping it with $<<$ Requirement $>>$ (5.2.9). The "ID" tagged value specified the unique ID of the requirement. The "Kind" tagged value is 
set to "Safety" to identify that this is a safety-related requirement, and its "Specification" tagged value states what the requirement is, namely ensuring "Safe Flight Paths".

Because of its high software level, steeringController is checked for correctness using a formal method, namely "Theorem-Proving". Therefore, it is stereotyped with $\ll$ Formalism $\gg$ (5.2.23) and its "Method" tagged value is set to "Theorem-Proving". Its "Kind" tagged value is set to "Full" to indicate that a fully formal method is used.

PilotKeyboardinterface serves as an input interface to the pilot. The pilot can use it to change the flight path of the aircraft. PilotKeyboardInterface has been assigned a safety requirement to request confirmation from the pilots whenever they wish to change the flight path. Therefore, it was stereotyped with $<<$ Requirement $>>$ (5.2.9). Its "ID" tagged value specifies the unique ID of the requirement, which is "LREQ 1". Its "Kind" tagged value was set to "Safety" to indicate that it is a safety requirement. Its "OfRequirement" tagged value specifies the high-level requirement that this low-level requirement can be traced to. In this example, it is the "HREQ 1" high-level requirement that was applied as a stereotype on the diagram. The "Specification" tagged value specifies that there must be a user confirmation for every path change.

The second safety requirement for "HREQ 1" was assigned to the association between AutoPilotController and SteeringController. The association was stereotyped with $<<$ Requirement $>>$ (5.2.9) whose "Kind" tagged value is also set to "Safety". Its "ID" tagged value specifies the unique ID of the requirement. The "OfRequirement" tagged value specifies that this requirement is traceable to the "HREQ 1" requirement. The "Specification" tagged value specifies that the association must ensure that the "Chosen 
Aircraft Flight Path is in Safe Flight Paths Set". Therefore, AutoPilotController must not command steeringcontroller to fly in a flight path that is not in the safe flight paths set.

AutoPilotcontroller determines whether a particular flight path is allowed or not by reading the data managed by SafeFlightPaths. The availability of such information is the only reason for the existence of SafeFlightPaths. Therefore, it was stereotyped with < Rationale>> whose "Reference" tagged value is set to "LREQ 2" to identify the requirement whose existence resulted developing this class.

Finally, notice that the language used in the "Specification" tagged value for the $<<$ Requirement $>>$ (5.2.9) stereotype is more detailed and specific for low-level requirements than for the high-level requirements. In fact, the "Specification" tagged values for the low-level requirements can be written as mathematical expressions.

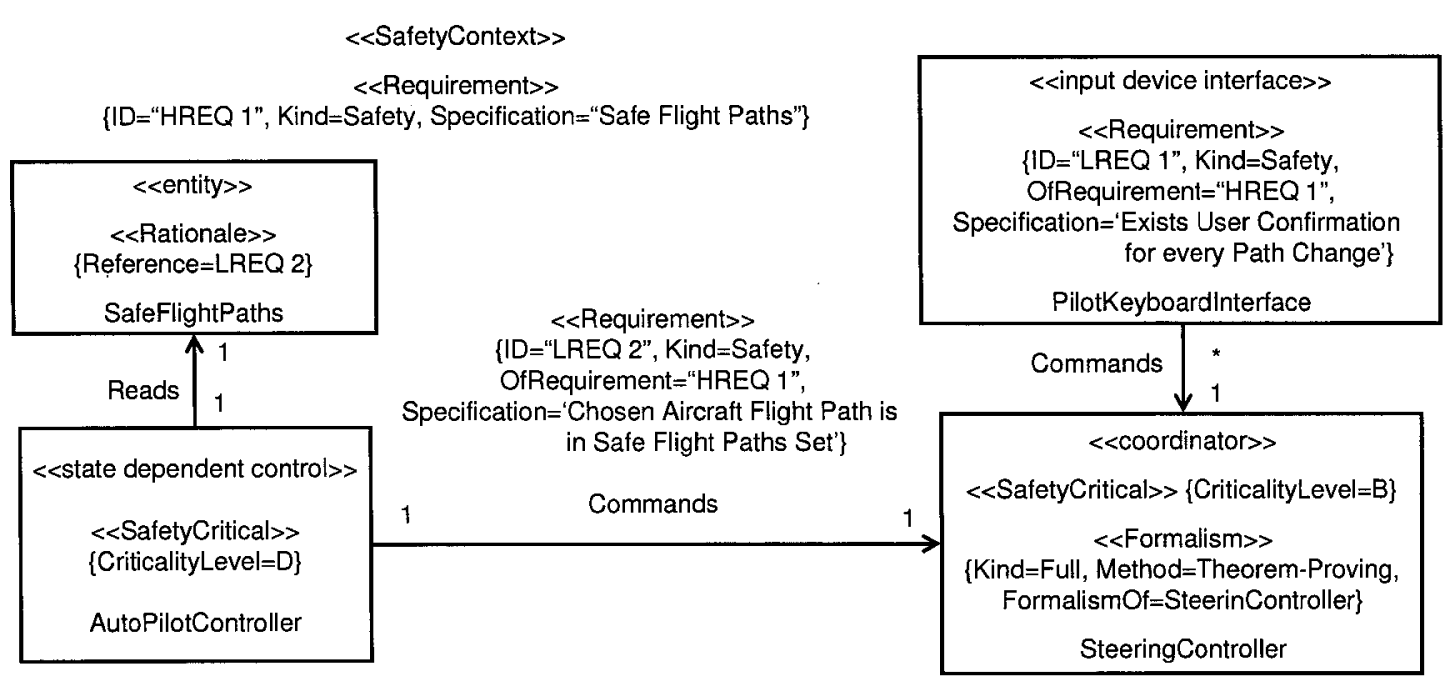

Figure 19: Safety-requirements for an aircraft steering system (structure). 


\section{G.4 Multiple-Version Dissimilar Software}

Multiple-version dissimilar software is a common technical solution to reliability challenges in highly reliable and safety-critical software. The example in Figure 20 shows three dissimilar software versions that function as a radar filter. Those three dissimilar versions are RadarFilter1, RadarFilter2, and RadarFilter3. Each one of them is stereotyped with $<<$ Replicated $>>$ to indicate that it is a dissimilar version for some other class. The "ID" tagged value uniquely identifies the ID of that class within the replication group that is specified in the "ReplicationGroup" tagged value. In this example, the replication group is called "RadarFilter".

Each version of the radar filter logs its output to RadarfilterResults. RadarfilterResults compares all three outputs from RadarFilterl, RadarFilter2, and RadarFilter3, and determines what the accepted value should be and then it updates the pilot's display accordingly. Because of this behaviour, RadarFilterResults is stereotyped with $<<$ Comparator $>>$ (5.2.32) to indicate that it compares outputs from dissimilar software versions. The "ReplicationGroup" tagged value identifies the replication group of the multiple version dissimilar software. It is equal to the "ReplicatedGroup" tagged values of the $<<$ Replicated $>>(5.2 .31)$ stereotypes for each of the replicated software versions. The "PolicyParameter" tagged value of the $<<$ Comparator $>$ stereotype indicates that RadarfilterResults determines the accepted output based on a majority voting policy. This means that if two of the three replicated classes agree on a value, their output is accepted as the correct one. The 
"ReplicatedEntity" tagged values of the <<Comparator $>>$ (5.2.32) stereotype identify the classes whose outputs are considered for voting.

Since multiple-version dissimilar software is a reliability-related solution, the diagram was stereotyped with $<<$ ReliabilityContext $>>(5.2 .2)$.

Notice that in this case, the classes stereotyped with $<<$ Replicated $>>$ (5.2.31) depend (navigability of association) on the one stereotyped with $<<$ Comparator $>$ (5.2.32). This is only because of a design decision in this model where the radar filters are active components that read the radar input and inform the comparator class accordingly. However, this will not always be the case. There can be cases where the comparator class depends on the replicated classes. Therefore, stereotypes <<comparator $>$ and $<<$ replicated $>>$ do not suggest any specific association navigability between stereotyped classes.

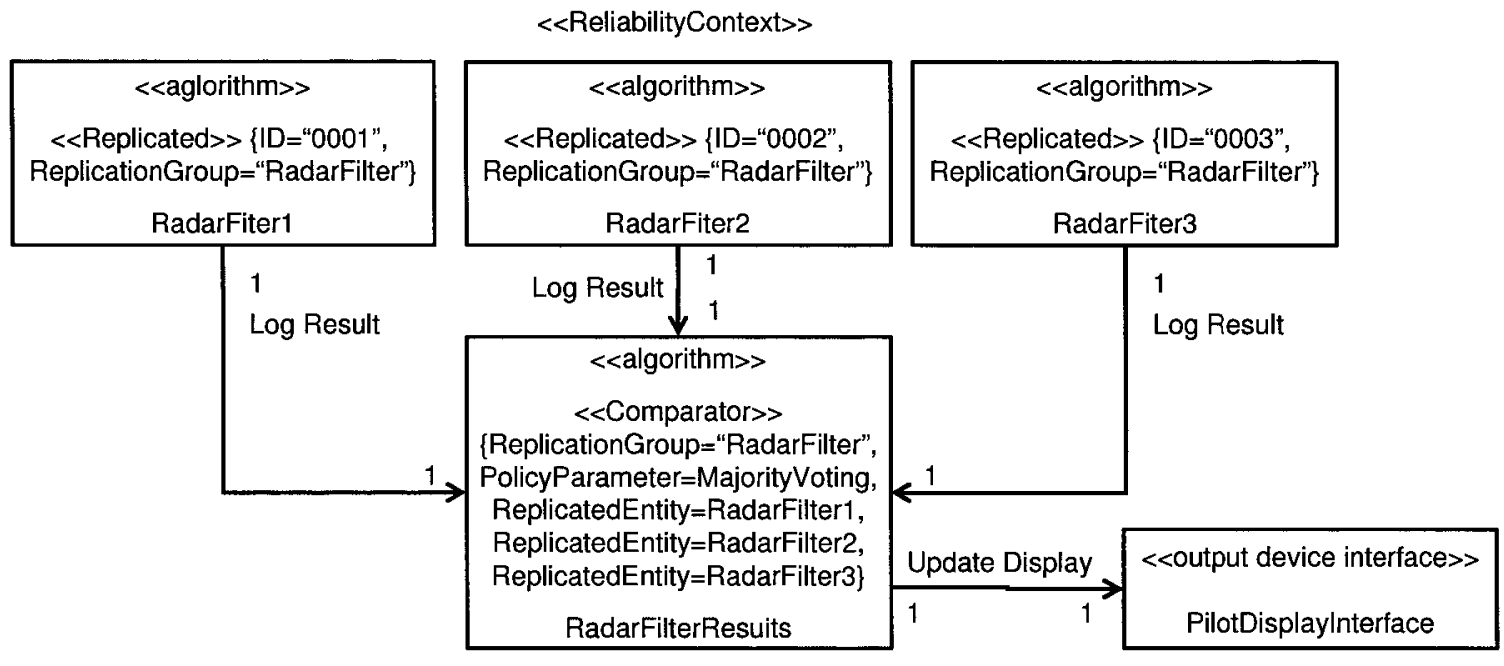

Figure 20: Multiple-version radar filter system (structure). 


\section{G.5 Concurrent Software}

This example shows concurrent access to the database presented in Figure 19. As Figure 21 shows, SafeFlightPaths is COTS software as indicated by the $<<$ Nature $>$ stereotype and its "Kind" tagged value. This class is a resource that is subject to concurrent access from other classes. Therefore, it is stereotyped with $<<$ Concurrent $>$ (5.2.26) to indicate that it is relevant from a concurrency point of view. Its "Role" tagged value is set to "Resource" and its "IsShared" "tagged value is set to "true" to indicate that it is a shared resource.

SafeFlightPaths is subject to concurrent access from SatellitecommunicationInterface, RadarInterface, and UserInterface. Each one of those classes is an active class that may initiate action without explicit invocation from other software classes in the system. Therefore, each one of them is stereotyped with $<<$ Concurrent $>>$ (5.2.26) whose "Role" tagged value was set to "Active". Each one of them has an association to SafeFlightPaths to show that it can read from and write to it.

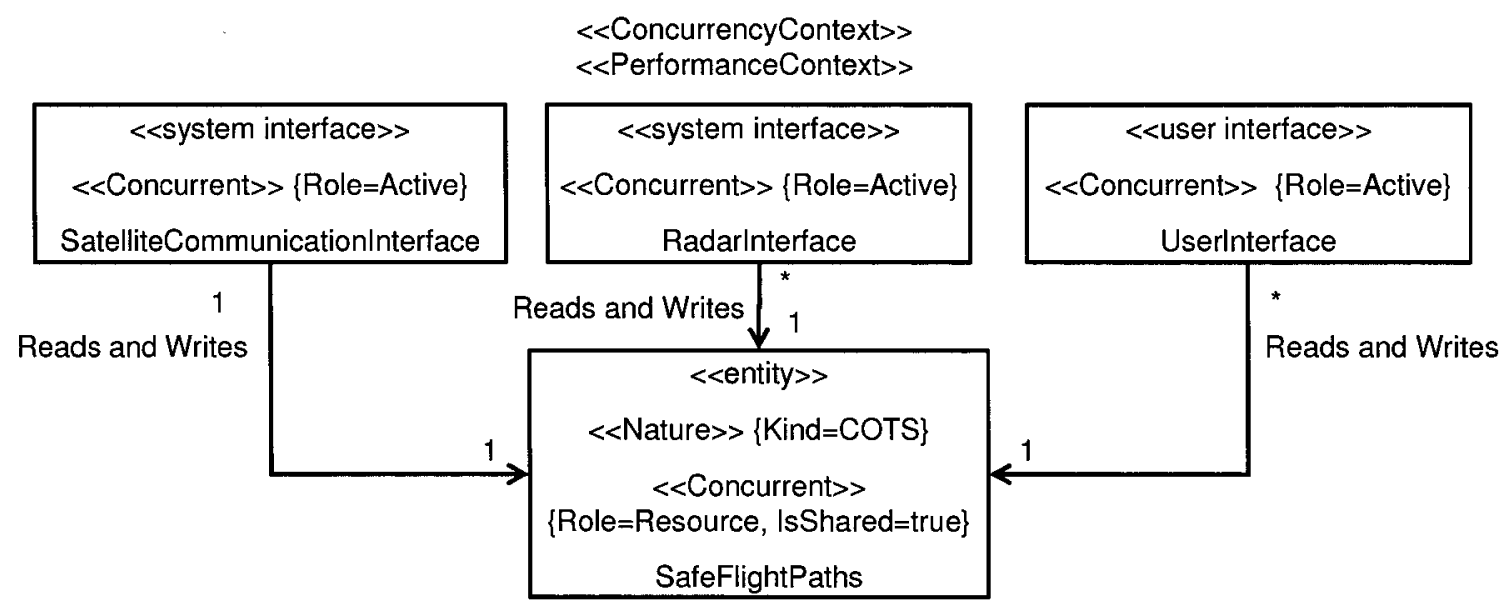




\section{Figure 21: Concurrent access to an aircraft's COTS software (structure).}

Because this diagram involves a concurrency discussion, it is stereotyped with $<<$ ConcurrencyContext $>$ (5.2.5). It is also stereotyped with $<<$ PerformanceContext $>>$ (5.2.4) because concurrent access to a shared resource is also relevant from a performance point of view especially because RadarInterface and SatellitecommunicationInterface are likely to have high-frequency accesses to SafeFlightPaths.

\section{G.6 Software Monitoring}

The example in Figure 22 shows safety-monitoring software. Its purpose is to detect when the aircraft's engine temperature becomes too high, and then to react by lowering the temperature to an acceptable level.

EngineInterface serves as an interface for the aircraft's engine. Hence, it is stereotyped with <<Interface>> (5.2.25) and its "InterfaceFor" tagged value is set to "AircraftEngine". Because the aircraft's engine is a hardware component, its "IsBetweenHardwareAndSoftware" tagged value is set to "true".

EngineInterface is monitored by EngineMonitor, which is stereotyped with $<$ Monitor > (5.2.20). Its "Kind" tagged value is set to "Safety" to indicate that the purpose of the monitor is to increase the safety level of the system. The "MonitoredEntity" tagged value is set to "EngineInterface" to indicate that the class being monitored is EngineInterface. The "DetectableEvent" tagged value specifies the event that can be detected by EngineMonitor, which is EngineTooHot. The 
"EventHandler" tagged value is set to "AircraftEngineController" to indicate that AircraftengineController will be notified when this event occurs.

EngineTooHot represents the event that the engine's temperature has risen to an unacceptable level. Thus, it is stereotyped with $\ll$ Event $>$ (5.2.15) and its "EffectOnSafetyDirection" tagged value is set to "Negative" to indicate that the occurrence of this event can have unsafe consequences.

NormalizeEngineTemperature contains the reaction code that will be executed when the EngineTooHot event occurs. Therefore, it is stereotyped with $<<$ Reaction $>>(5.2 .16)$ and its "ConsequenceOf" tagged value is set to "EngineTooHot", which is the class name of the event that triggers the reaction. The "EffectOnSafetyDirection" is set to "Positive" to indicate that the reaction is intended to increase the safety level.

AircraftEngineController is a safety-critical class because it determines how to control the aircraft's engine. Thus, it is stereotyped with $<<$ SafetyCritical $>$ (5.2.17). Moreover, it serves as an event handler by recognizing the EngineTooHot event and executing the NormalizeEngineTemperature reaction code. Therefore, it is also stereotyped with $<<$ Handler $>$ (5.2.19) stereotype whose "HandleableEvent" tagged value is set to the "EngineTooHot" event, and its "PerformedReaction" tagged value is set to the "NormalizeEngineTemperature" reaction.

Because this diagram discusses safety aspects of the system, it is stereotyped with $<<$ SafetyContext $>>$ (5.2.1). Furthermore, it is stereotyped with $<<$ Strategy $>>(5.2 .22)$ and its "Kind" tagged value is set to "Safety" to indicate that it is a technical solution to increase the safety level. 


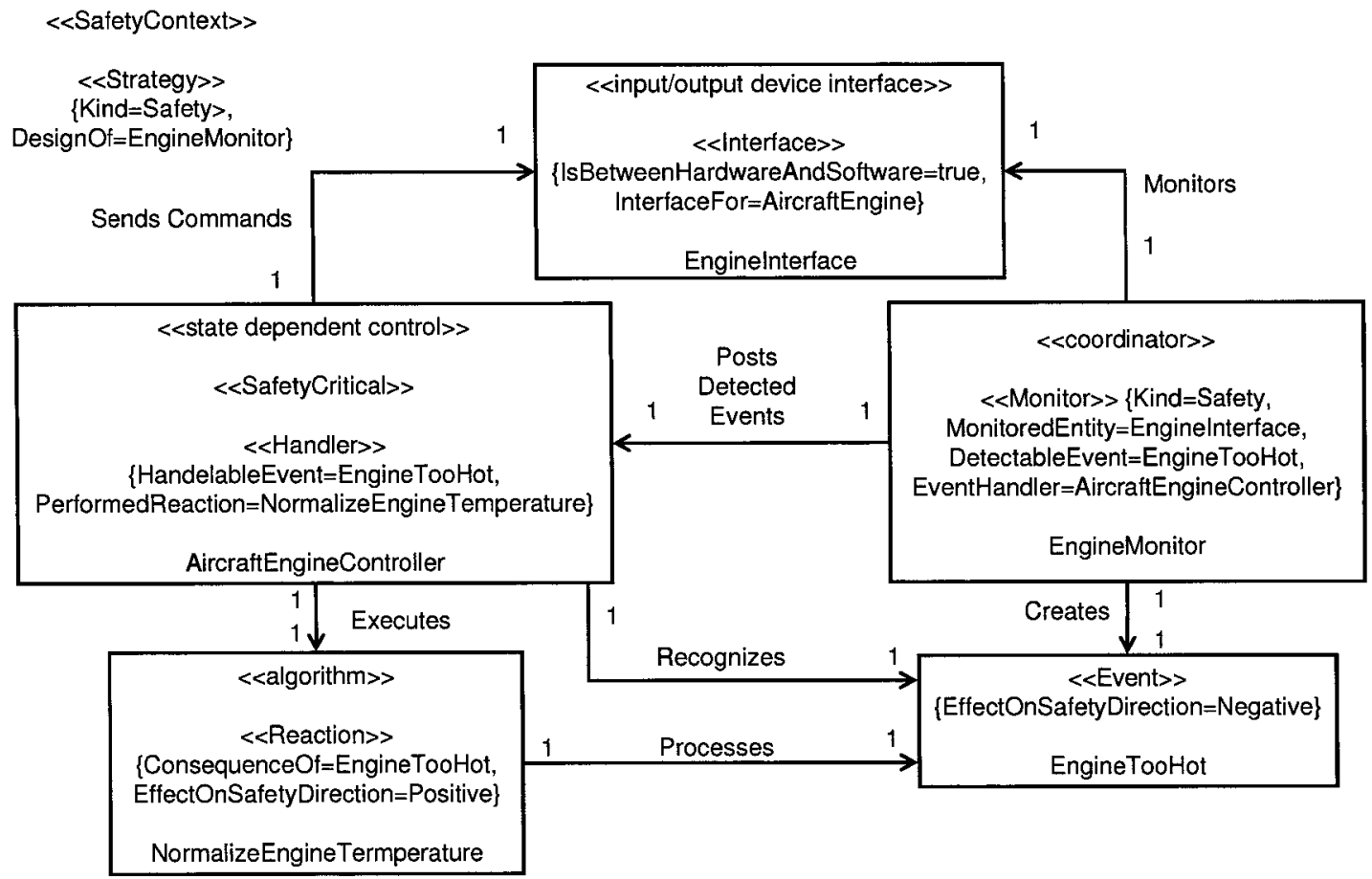

Figure 22: Monitoring the aircraft's engine's temperature (structure). 